\title{
WATER-QUALITY DATA FOR THE OHIO RIVER FROM WILLOW ISLAND DAM TO BELLEVILLE DAM, WEST VIRGINIA AND OHIO, JUNE-OCTOBER 1995
}

By Kimberly F. Miller and John T. Atkins

U.S. GEOLOGICAL SURVEY

Open-File Report 97-565

Prepared in cooperation with the

CITY OF NEW MARTINSVILLE, WEST VIRGINIA

Charleston, West Virginia

1997

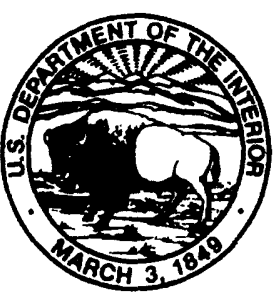




\section{U.S. DEPARTMENT OF THE INTERIOR \\ BRUCE BABBITT, Secretary \\ U.S. GEOLOGICAL SURVEY}

Gordon P. Eaton, Director

For additional information write to:

District Chief

U.S. Geological Survey, WRD

11 Dunbar Street

Charleston, WV 25301
Copies of this report can be purchased from:

U.S. Geological Survey

Branch of Information Services

Box 25286

Denver, CO 80225-0286 


\section{CONTENTS}

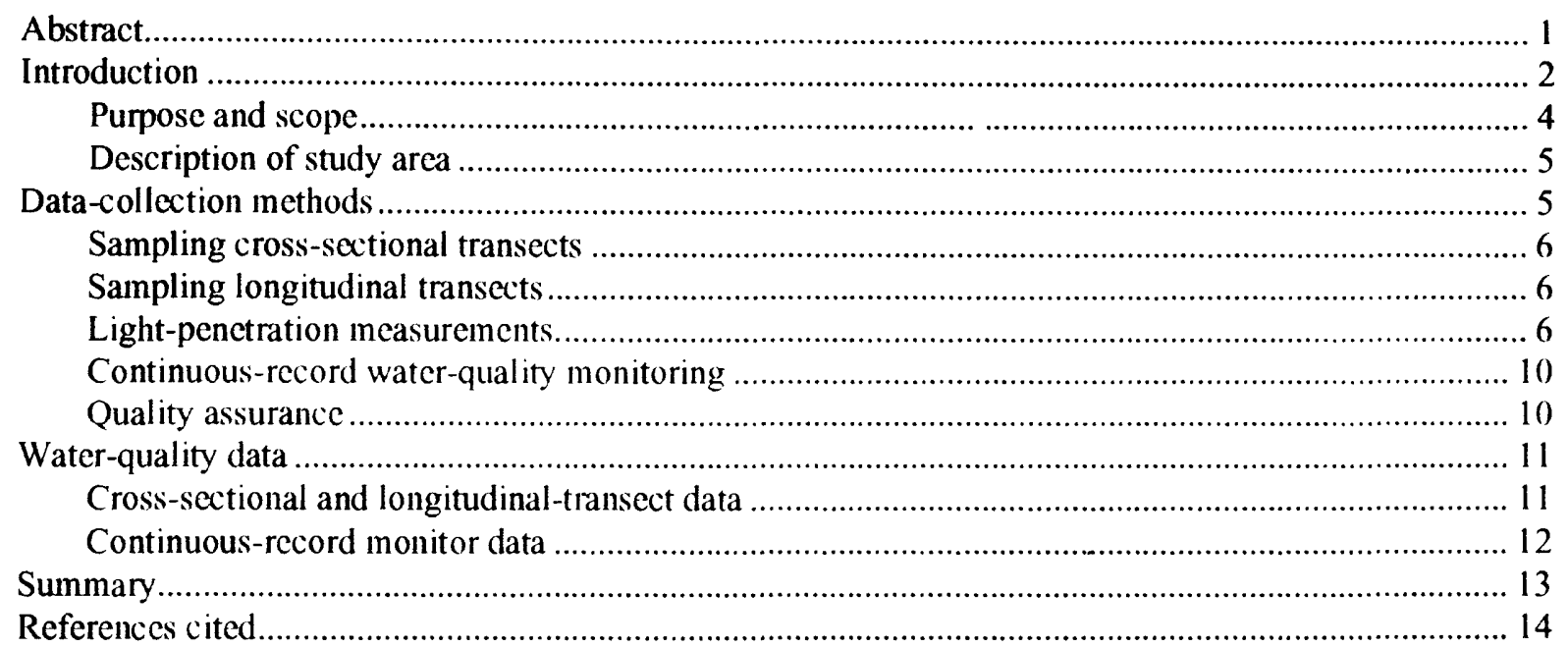

\section{FIGURES}

1-2. Maps showing:

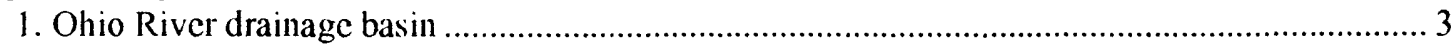

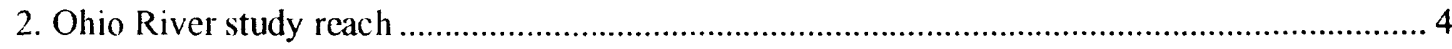

3a. Eastern section of study reach with water-quality sampling sites and cross sections....................... 7

3b. Middle section of study reach with water-quality sampling sites and cross sections ....................... 8

3c. Western section of study reach with water-quality sampling sites and cross sections........................ 9

4. Diagram of Willow Island Dam showing locations of continuous-recording water-quality monitors..10

\section{TABLES}

1-30. Water-quality data, June to October 1995, for:

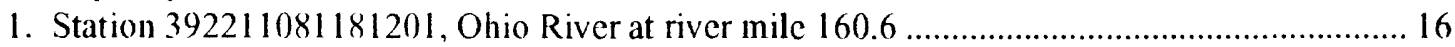

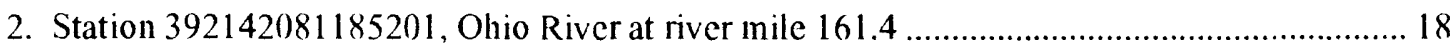

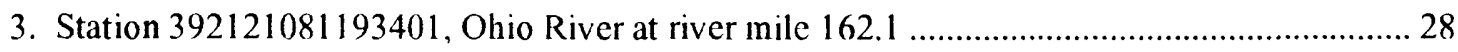

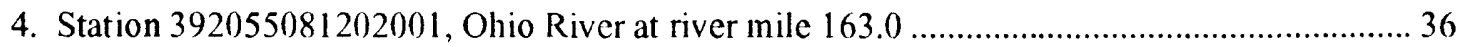

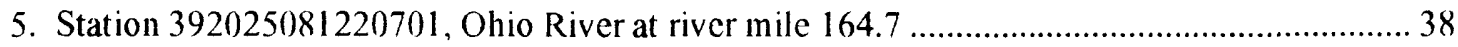

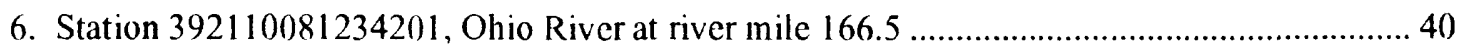

7. Station 392318081243001 , Ohio River at river mile 169.1, main channel ...............................42

8. Station 392313081244601 , Ohio River at river mile 169.1, back channel ................................ 44

9. Station 392419081255001, Ohio River at river mile 170.8, main channel...............................46

10. Station 39241108125590 1, Ohio River at river mile 170.8, back channel ............................... 48

11. Station 392232081295601 , Ohio River at river mile 175.5, main channel ............................... 50

12. Station 392227081293701, Ohio River at river mile 175.5, back channel .............................. 52

13. Station 392139081312801, Ohio River at river mile 177.2, main channel .............................. 54

14. Station 392131081312301, Ohio River at river mile 177.2, back channel .............................. 56

15. Station 392042081330101, Ohio River at river mile 179.0) .............................................. 58 


\section{TABLES--Continued}

1-30. Water-quality data, June to October 1995 , for--

16. Station 391822081334701 , Ohio River at river mile 181.8

17. Station 391720081334701 , Ohio River at river mile 183.0

18. Station 391559081341201 , Ohio River at river mile 184.6

19. Station 391628081360401 , Ohio River at river mile 186.5, main channel .............................. 86

20. Station 391604081361301 , Ohio River at river mile 186.5, back channel ............................. 88

21. Station 391636081384701, Ohio River at river mile 189.0, main channel ............................90

22. Station 391616081385001, Ohio River at river mile 189.0, back channel ............................ 92

23. Station 391601081411101, Ohio River at river mile 191.3 ….......................................... 94

24. Station 391447081414201, Ohio River at river mile 192.9 …............................................ 96

25. Station 391351081412201, Ohio River at river mile 194.0 ........................................ 109

26. Station 391302081425101, Ohio River at river mile 195.8 ......................................... 111

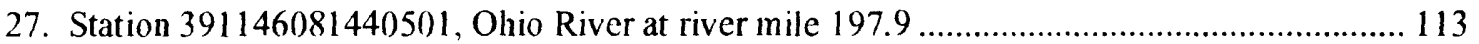

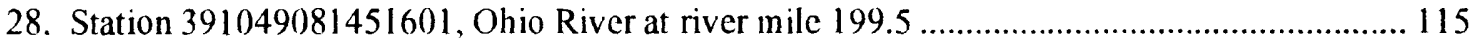

29. Station 390803081443501, Ohio River at river mile 202.8 ........................................... 117

30. Station 390721081443001 , Ohio River at river milc 203.6 .............................................. 119

31. Daily maximum, minimum, and mean specific conductance at station 392145081185601 , from the Willow Island Dam (upstream) continuous-recording water-quality monitor, June to October 1995

32. Daily maximum, minimum, and median $\mathrm{pH}$ at station 392145081185601 , from the Willow Island Dam (upstream) continuous-recording water-quality monitor, June to October 1995.

33. Daily maximum, minimum, and mean water temperature at station 392145081185601, from the Willow Island Dam (upstream) continuous-recording water-quality monitor, June to October 1995.

34. Daily maximum, minimum, and mean dissolved oxygen concentrations at station 392145081185601, from the Willow Island Dam (upstream) continuous-recording water-quality monitor, June to October 1995.

35. Daily maximum, minimum, and mean specific conductance at station 392125081193601, from the Willow Island Dam (downstream) continuous-recording water-quality monitor, June to October 1995.

36. Daily maximum, minimum, and median $\mathrm{pH}$ at station 392125081193601 , from the Willow Island Dam (downstreain) continuous-recording water-quality monitor, June to October 1995.

37. Daily maximum, minimum, and mean water temperature at station 3921250811936) I, from the Willow Island Dam (downstream) continuous-recording water-quality monitor, June to October 1995.

38. Daily maximum, minimum, and mean dissolved oxygen concentrations at station 392125081193601, from the Willow Island Dain (downstream) continuous-recording water-quality monitor, June to October 1995. 


\title{
WATER-QUALITY DATA FOR THE OHIO RIVER FROM WILLOW ISLAND DAM TO BELLEVILLE DAM, WEST VIRGINIA AND OHIO, JUNE-OCTOBER 1995
}

\author{
By Kimberly F. Miller and John T. Atkins
}

\begin{abstract}
This report contains water-quality data for the Ohio River from river mile $160.6(1.1 \mathrm{mi}$ upstream from Willow Island Dam) to river mile 203.6 (0.3 mi upstream from Belleville Dam) that were collected during the summer and fall 1995. The data were collected to define the water quality of the Ohio River and to use in assessing the proposed effects of hydropower development on the water quality of the Ohio River. Water quality was determined by a combination of synoptic field measurements and continuous-record monitoring. Field measurements of waterquality characteristics were made along a longitudinal transect with 24 mid-channel sampling sites; cross-sectional transects of water-quality measurements were made at 6 of these sites. Water-quality measurements also were made at six sites located on the back-channel (West Virginia) sides of Marietta, Muskingum, and Blennerhassett Islands. At each longitudinaltransect and back-channel sampling site, measurements of specific conductance, $\mathrm{pH}$, water temperature, and dissolved-oxygen concentration were made at four depths (surface of the water, about 3.0 feet below the surface, middle of the water column, and near the bottom of the river) constituting a four-point vertical profile. Cross-sectional transects consisted of three or four detailed vertical profiles of the same characteristics. Estimates of the depth of light penetration (Secchi disk transparency) were made at cross-sectional sampling locations whenever light and river-surface conditions were appropriate. Each synoptic sampling period was completed in two days or less. The entire network was sampled nine times from June 28 to October 19, 1995.

Continuous-record monitoring of water quality consisted of hourly measurements of specific conductance, $\mathrm{pH}$, water temperature, and dissolved oxygen concentration, that were recorded at a depth of approximately 6.6 feet at the ends of the upstream and downstream wingwalls at Willow Island Dam. Continuous-recording monitors were operated from June through October 1995. Maximum and minimum measurements at the upstream monitor for specific conductance were 667 microsiemens per centimeter and 260 microsiemens per centimeter, for $\mathrm{pH}$ were 8.4 and 7.0 , for water temperature were $32.4^{\circ} \mathrm{Celsius}$ and $15.8^{\circ} \mathrm{Celsius,}$ and for dissolved oxygen were 11.5 milligrams per liter and 5.3 milligrams per liter, respectively. The downstream monitor maximum and minimum measurements for specific conductance were 675 microsiemens per centimeter and 257 microsiemens per centimeter, for $\mathrm{pH}$ were 8.1 and 7.1 , for water temperature were $31.2^{\circ}$ Celsius and $16.0^{\circ}$ Celsius, and for dissolved oxygen 10.7 milligrams per liter and 5.6 milligrams per liter, respectively.
\end{abstract}




\section{INTRODUCTION}

The U.S. Army Corps of Engineers has constructed and operates more than 60 lock-anddam facilities in the Ohio River Basin, with 20 facilities on the Ohio River mainstem and the rest on major tributaries in the basin (U.S. Army Corps of Engineers, 1990). The lock-and-dam structures form a system of contiguous navigation pools that ensure year-round navigation on the river. Many dams also contain hydroelectric generators that were installed after construction of the navigation structures. In 1989, the Federal Energy Regulatory Commission (FERC) issued licenses for retrofitting of hydropower at 19 dams in the upper Ohio River Basin, which includes the Allegheny and Monongahela Rivers, and the Ohio River mainstem from Pittsburgh, Pennsylvania, to Huntington, West Virginia (fig. 1). However, many of these licenses have since been surrendered, indicating that perhaps the original licensees do not intend to develop projects.

Some dams scheduled for hydropower development currently are thought to improve the quality of the river by increasing the rate of gas transfer from the atmosphere to the water (Federal Energy Regulatory Commission, 1988). Water from deep, slow-moving upstream pools is mixed as it passes over or through navigation structures, and the amount of surface area in contact with the atmosphere is increased. If the dissolved-oxygen (DO) concentration is less than the saturation concentration, the potential exists for absorption of oxygen into the water, a process known as reaeration.

The amount of oxygen added to the water by reaeration at a dam depends, in part, on flow conditions and design characteristics of the structure (Avery and Novak, 1978). Dams on the upper Ohio River downstream from Wheeling, W. Va., are gated structures that discharge several feet below the surface of the downstream pool and so provide little reaeration (Federal Energy Regulatory Commission, 1988). Other dams, including overflow dams and gated dams with discharge above the downstream pool level, are more efficient aerators and can be important sources of DO during low-flow conditions of summer and early fall. Dams upstream from Wheeling are of the latter type. Hydropower operation at these surface-discharging structures will divert riverflow through underwater intakes where the opportunity for atmospheric gas exchange is smaller. For dams upstream from Wheeling, the loss of reaeration at low flows, combined with the oxygen consumption associated with waste assimilation and the failure of other oxygen-generating processes such as algal photosynthesis, could reduce DO concentrations below acceptable levels and diminish the wasteassimilation capacity of the river (West Virginia Department of Natural Resources, 1989).

A water-quality monitoring program was begun in 1991 in cooperation with the city of New Martinsville, W. Va., and was designed, in part, to address license requirements for development of hydropower at Willow Island Dam (FERC Project No. 6902). This dam is located upstream from Parkersburg, W. Va., and is of the deep-discharge type. The program uses continuous-record monitoring and synoptic sampling of water-quality characteristics near the dam and throughout the downstream navigation pool during the summer and fall to provide basic hydrologic and ecological data on the possible environmental effects of hydropower operation. Synoptic surveys, where water-quality characteristics are analyzed quickly at many locations and depths, have been recommended for incorporation into water-quality impact assessments of proposed hydropower projects at dams and other control structures (Gulliver and others, 1990; Daniil and others, 1991).

The study described in this report was conducted in the Belleville navigation pool, a 43mi section of the Ohio River that begins at river mile 160.6 (1.1 mi upstream from Willow Island Dam) and extends downstream to river mile 203.6 (0.3 mi upstream from Belleville Dam) (fig. 2). The final environmental impact statement for development of hydropower at Willow Island Dam concluded that the dam provides little reaeration to the downstream pool (Federal Energy Regulatory Commission, 1988. p. 3-64). 


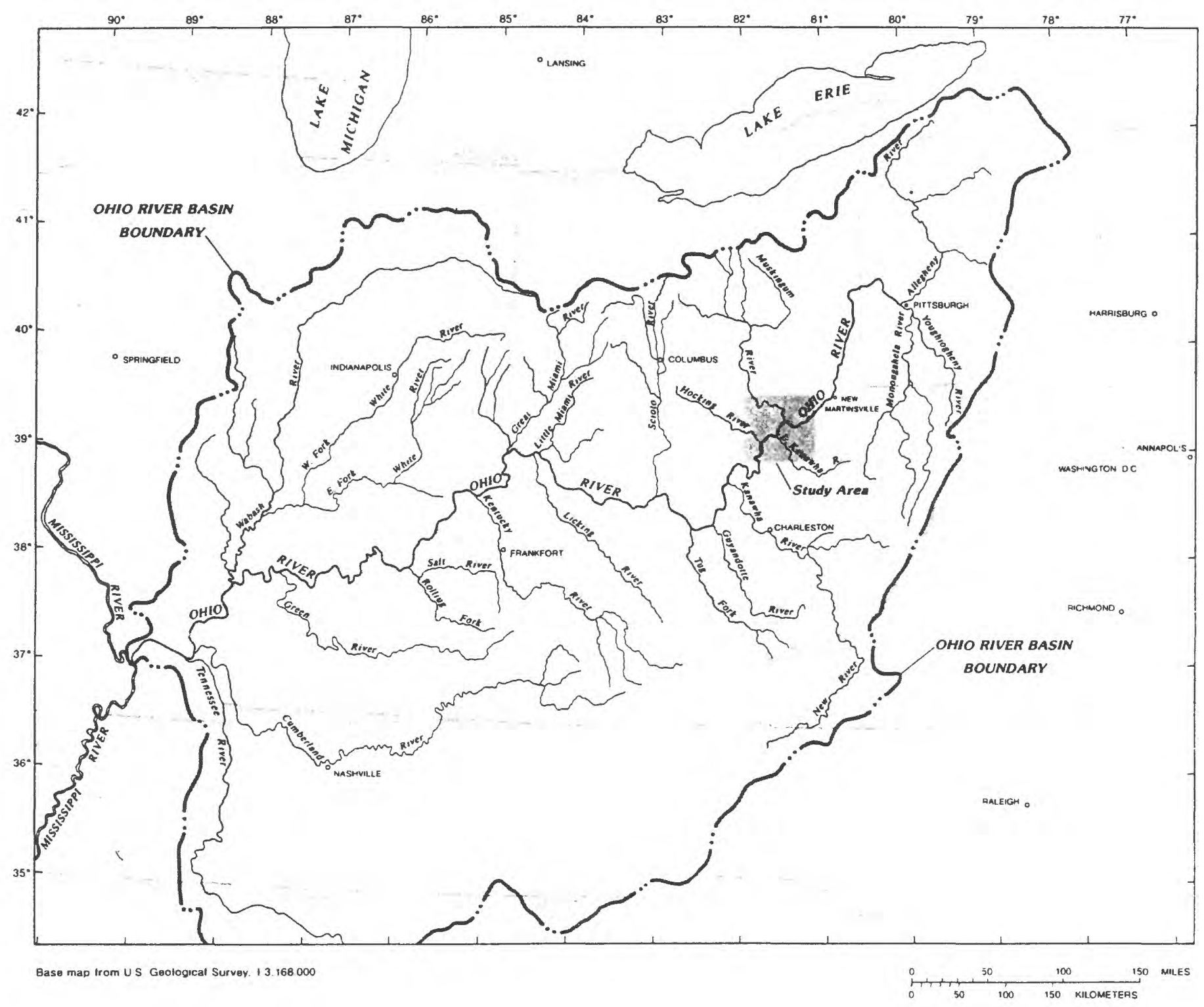

Figure 1. Ohio River drainage basin. 


\section{Description of Study Area}

Drainage area for the Ohio River at Belleville Dam is $39,300 \mathrm{mi}^{2}$. Most of the drainage basin up to the dam consists of narrow flood plains and deeply incised tributary valleys. Major tributaries in the study reach include the Muskingum River (drainage area: $8,040 \mathrm{mi}^{2}$ ), the Little Kanawha River (drainage area: 2,320 $\mathrm{mi}^{2}$ ), and the Hocking River (drainage area: $1,190 \mathrm{mi}^{2}$ ) (fig. 2). The basin is underlain by bedrock that consists of shale, sandstone, siltstone, limestone, and coal (West Virginia Department of Natural Resources, 1988). The average width of the Belleville pool is 1,327 $\mathrm{ft}$. The average bottom slope is $0.5 \mathrm{ft} / \mathrm{mi}$ (Ohio River Valley Water Sanitation Commission, 1988). Although the average depth of the pool is $24 \mathrm{ft}$, the depth of the main channel increases with distance downstream from the dam.

The climate of the region is considered temperate with distinct seasonal changes. Mean minimum air temperatures $\left(-4.8^{\circ} \mathrm{C}\right)$ are generally recorded during January; mean maximum air temperatures $\left(30.2^{\circ} \mathrm{C}\right)$ are generally recorded during July. Average annual air temperature is about $12^{\circ} \mathrm{C}$. Annual precipitation in the basin ranges from 20 to 72 in., with heaviest amounts occurring in June or July and minimum amounts occurring in October (West Virginia Department of Natural Resources, 1988). The U.S. Army Corps of Engineers has constructed a system of multipurpose reservoirs on four main tributaries for flood control. These reservoirs also are used to augment flow, and maintain navigation during critical periods.

Land use in the study reach is about 16 percent cropland, 12 percent pasture, 46 percent forest, 6 percent urban, and 20 percent other uses (Ohio River Valley Water Sanitation Commission, 1988). Major urban and industrial centers in the reach include Parkersburg, W. Va., and Marietta, Ohio (fig. 2). The reach includes one municipal drinking-water intake (Ranney well at Parkersburg) and seven industrial water intakes. Industrial activity along the reach is associated mainly with chemical manufacturing and coal-fired electric-power generation. This section of the river is also used to transport petroleum products, chemicals, and other materials. There are 16 river terminals in the study reach, most of which are located between Marietta and Parkersburg (Ohio River Valley Water Sanitation Commission, 1988). The States of West Virginia and Ohio have issued permits for 8 municipal and 22 industrial effluent discharges in the study reach.

\section{DATA-COLLECTION METHODS}

Water quality of the Belleville pool was determined by a combination of synoptic field measurements, laboratory analyses, and continuous monitoring. Field measurements were made on June 28, July 13, July 27, August 24, September 6, September 20, October 5, and October 19, 1995.

A partial set of field data was collected on August 10, 1995. Two continuous-recording monitors were in operation at Willow Island Dam from June 1 through October 31, 1995.

The field-data-collection network for the study consisted of a longitudinal transect with 24 mid-channel sampling sites; cross-sectional transects of water-quality characteristics were made at 6 of these sites. Three of these crosssectional transects (river miles 183.0, 184.6, and 192.9) had water-quality measurements taken at near sunrise and during the afternoon of the same day. Water-quality measurements also were made at six sites located on the back-channel (West Virginia) sides of the three largest islands (Marietta, Muskingum, and Blennerhassett) in the Belleville pool. Measurements made at each longitudinal-transect and back-channel sampling site included vertical profiles of specific conductance, $\mathrm{pH}$, water temperature, and $\mathrm{DO}$ concentration, with readings taken at the surface of the water, $3 \mathrm{ft}$ from the surface, from the middle of the water column, and at the bottom of the river. Cross-sectional transects consisted of three or four detailed vertical profiles of the same characteristics. Estimates of the depth of light penetration (Secchi disk transparency) were made at cross-sectional sampling locations whenever light and river-surface conditions were appropriate. 


\section{Sampling Cross-Sectional Transects}

During each sampling period, water-quality measurements were made in cross-sectional transects at six locations shown in figures 3a-3c. Two cross sections were located near Willow Island Dam at the ends of the upstream (river mile 161.4) and downstream (river mile 162.1) wingwalls (fig. 3a). Additional cross sections were located at Parkersburg, W. Va. (river mile 183.0), near the mouth of the Little Kanawha River (river mile 184.6), and at the downstream end of a large chemical manufacturing complex near Little Hocking, Ohio (river mile 192.9) (figs. 3b and 3c). One cross section was located at Belleville Dam at the end of the upstream wingwall (river mile 203.6) (fig. 3c). As weather permitted, the cross sections at river miles 183.0, 184.6, and 192.9 consisted of near-sunrise cross-sectional transect measurements of water quality. These same sampling sites were also measured during the afternoon of the same day of the near-sunrise measurements.

Cross-sectional transects at the Willow Island Dam and Belleville Dam sites usually consisted of four vertical profiles of specific conductance, $\mathrm{pH}$, water temperature, and DO concentration measurements. Positions of the vertical profiles were located by estimating $25,50,75$ and 100 percent of the distance from the left bank to the edge of the wingwall and were sampled in random order to minimize the effects of diel changes (changes associated with a 24-hour period which includes both day and night). Cross-sectional transects at other locations consisted of three vertical profiles, with positions determined by estimating 25,50 , and 75 percent of the total width of the river. Weather and river-surface conditions occasionally prevented completion of all vertical profiles in a transect. Complete vertical-profile measurements were made at the surface of the water, about 3.0 and $5.0 \mathrm{ft}$ below the surface, and then at depth intervals of about $5.0 \mathrm{ft}$ until just off of the bottom of the river, using a portable, multiparameter water-quality monitoring system (Hydrolab ${ }^{l}$ Surveyor 3). Measuring was begun either at the bottom of the river or at the surface. Barometric pressure was recorded before each set of field measurements by use of a Thommen TX altimeter-barometer.

\section{Sampling Longitudinal Transects}

Longitudinal transects consisted of measurements of specific conductance, $\mathrm{pH}$, water temperature, and DO concentration made at four depths (surface of the water, about $3.0 \mathrm{ft}$ below the surface, middle of the water column, and near the bottom of the river) at 24 mid-channel sampling sites distributed throughout the Belleville pool. A complete depth profile (surface of the water, $3.0 \mathrm{ft}$ below the surface, and then at intervals of $5.0 \mathrm{ft}$ down to the bottom of the river) was measured at three of the original 24 mid-channel longitudinal sampling sites (river miles 160.6, 163.0, and 202.8). Six additional sampling sites were located on the back-channel (West Virginia) sides of Marietta, Muskingum, and Blennerhassett Islands. The locations of the sampling sites are shown in figures $3 a-3 c$. Each location corresponds to the position of a U.S. Coast Guard navigation light or daymark. Sampling methods and instruments were the same as for the crosssectional transects.

\section{Light-Penetration Measurements}

At each cross-sectional sampling site, an estimate of the depth of light penetration was made lowering a 9-in.-diameter Secchi disk into the water until the disk was no longer visible from the surface, and recording the depth. All Secchi disk measurements were made between the hours of 1000 and 1600 Eastern Daylight Savings Time (EDT). Secchi-disk depths were not recorded if the sampling time was outside this time window or if high surface waves made accurate measurement impossible.

\footnotetext{
1. The use of brand, firm, or trade names in this report is for identification purposes and does not constitute endorsement by the U. S. Geological Survey.
} 


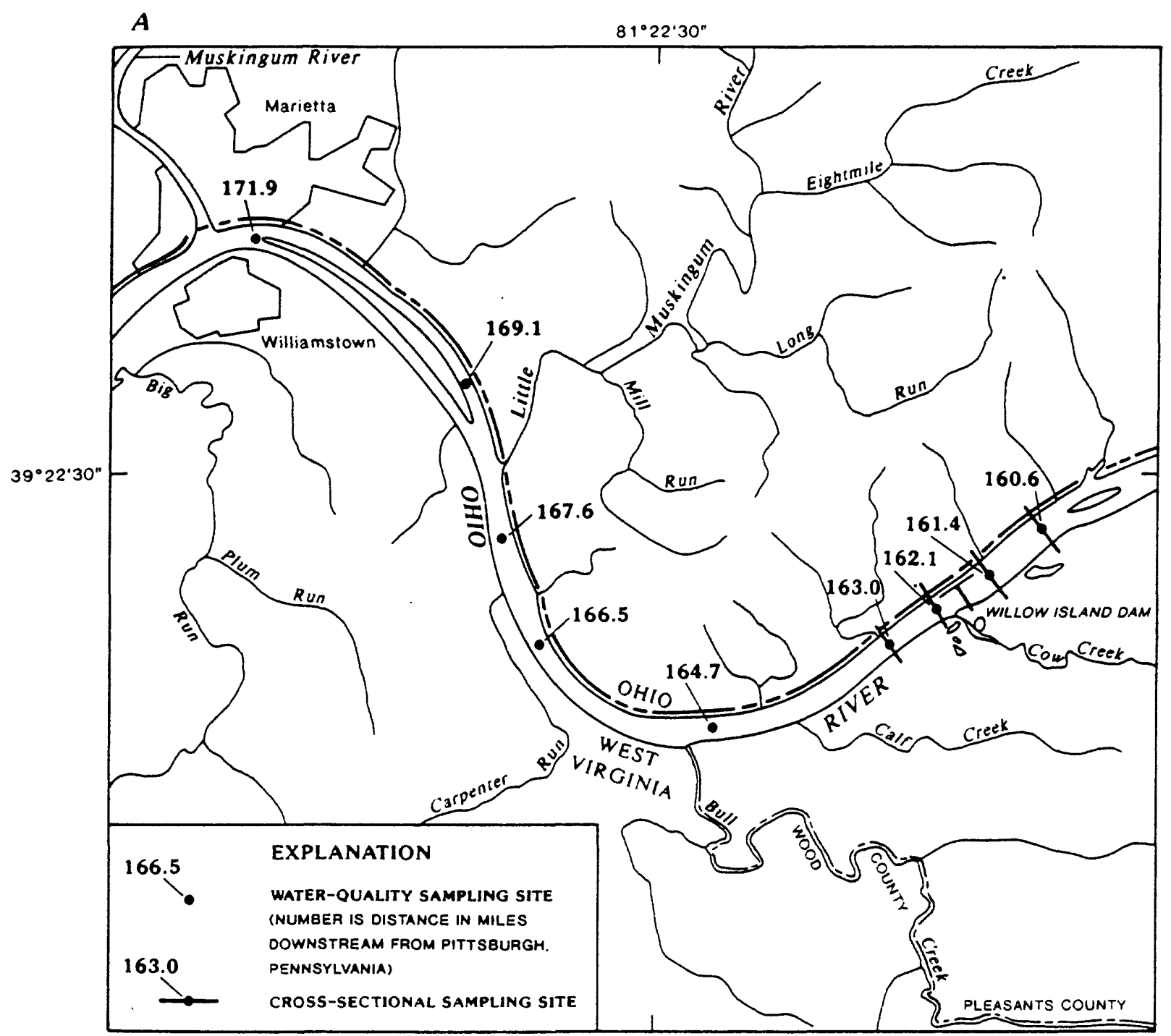

Base map trom US Geological Survey. $1 \cdot 100.000$

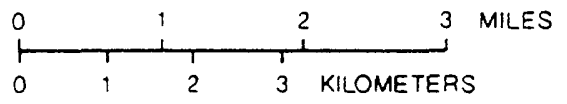

Figure 3a. Water-quality and cross-sectional sampling sites in the eastern (upstream) section of the study reach. 


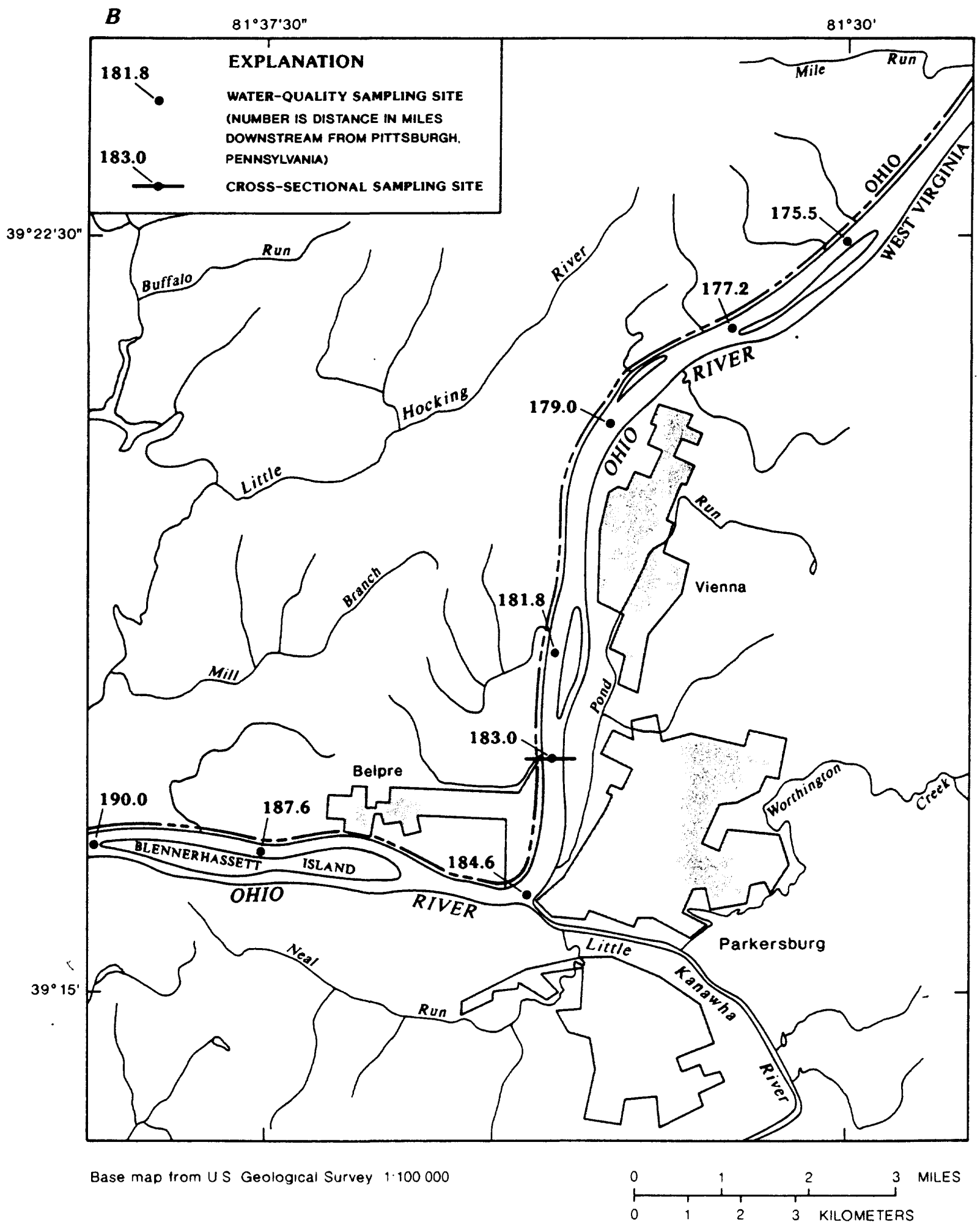

Figure 3b. Water-quality and cross-sectional sampling sites in the middle section of the study reach. 


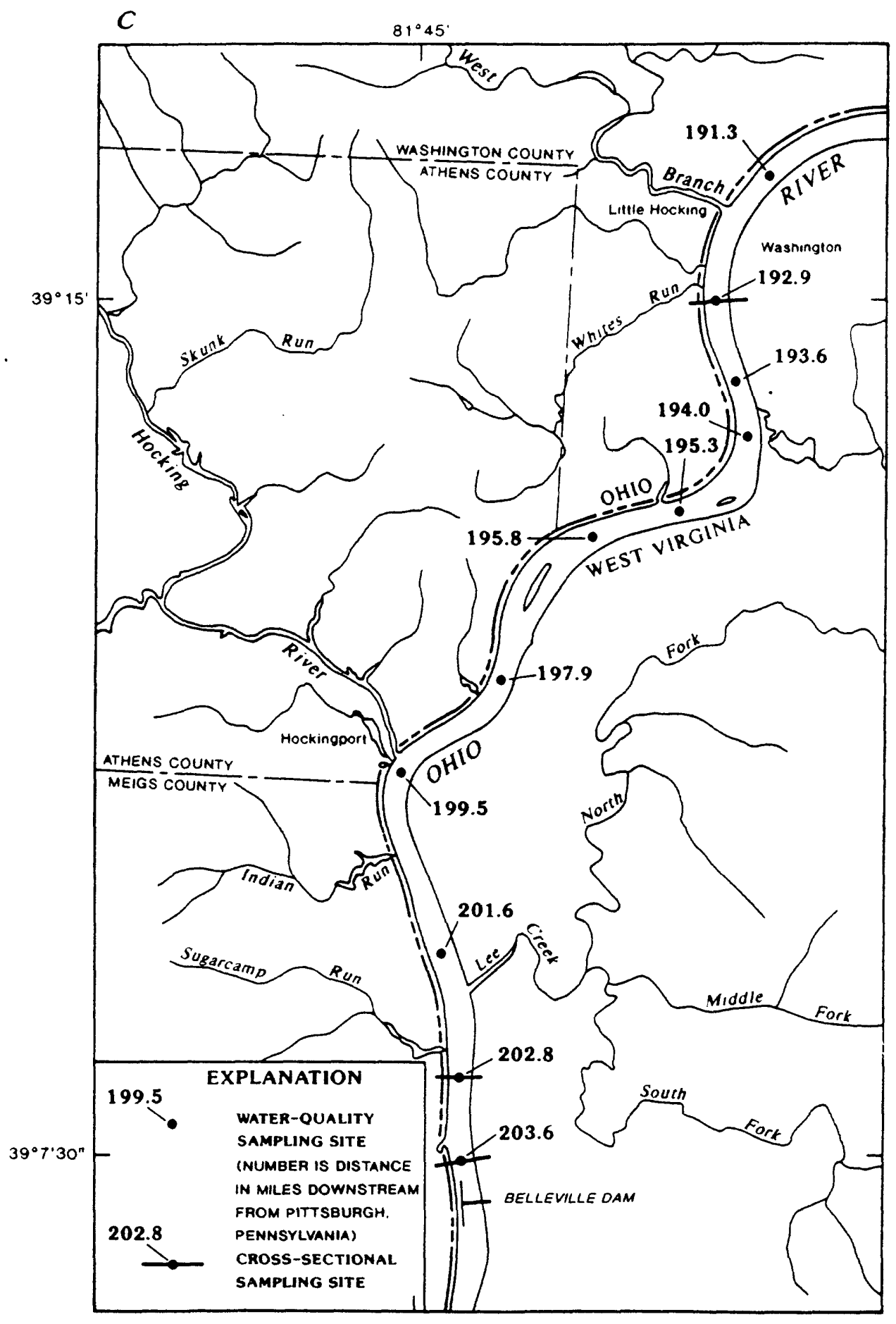

Base map from U.S Geological Survey $1 \cdot 100.000$

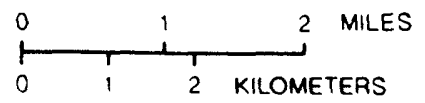

Figure 3c Water-quality and cross-sectional sampling sites in the western (downstream) section of the study reach. 


\section{Continuous-Record Water-quality Monitoring}

Continuous-recording water-quality monitors were installed in June 1995 at sites upstream and downstream from Willow Island Dam (fig. 4). The monitors consisted of Hydrolab H20 multiparameter data transmitters connected to Handar $570 \mathrm{~A}$ data-collection platforms that recorded hourly measurements of specific conductance, $\mathrm{pH}$, water temperature, and DO concentration, and transmitted data at 4-hour intervals by way of the Geostationary Operational Environmental Satellite (GOES). The upstream monitor was located at the end of the upstream wingwall on the riverside, about $1,200 \mathrm{ft}$ from the dam (latitude $39^{\circ} 21^{\prime} 45^{\prime \prime} \mathrm{N}$, longitude $81^{\circ} 18^{\prime} 56^{\prime \prime} \mathrm{W}$ ), in a section of 6 -in. PVC pipe at a fixed depth of $6.6 \mathrm{ft}$. The downstream monitor was located in a similar position at the end of the downstream wingwall.

\section{Quality Assurance}

The portable water-quality monitoring system was calibrated at the beginning of each sampling period in accordance with the recommendations of the manufacturer (Hydrolab Corporation, 1991), and all parameters were checked periodically during the day for meter drift. Barometric pressure was recorded before each set of field measurements by use of an analog barometer that was calibrated against a mercury barometer maintained by the National Weather Service Forecast Office in Charleston, W. Va.

The portable monitoring system measures $\mathrm{DO}$ concentration electrometrically with a standard membrane electrode. The electrode was calibrated by reading the meter against water-saturated air at known temperature and barometric pressure. As a further check of the accuracy of the DO

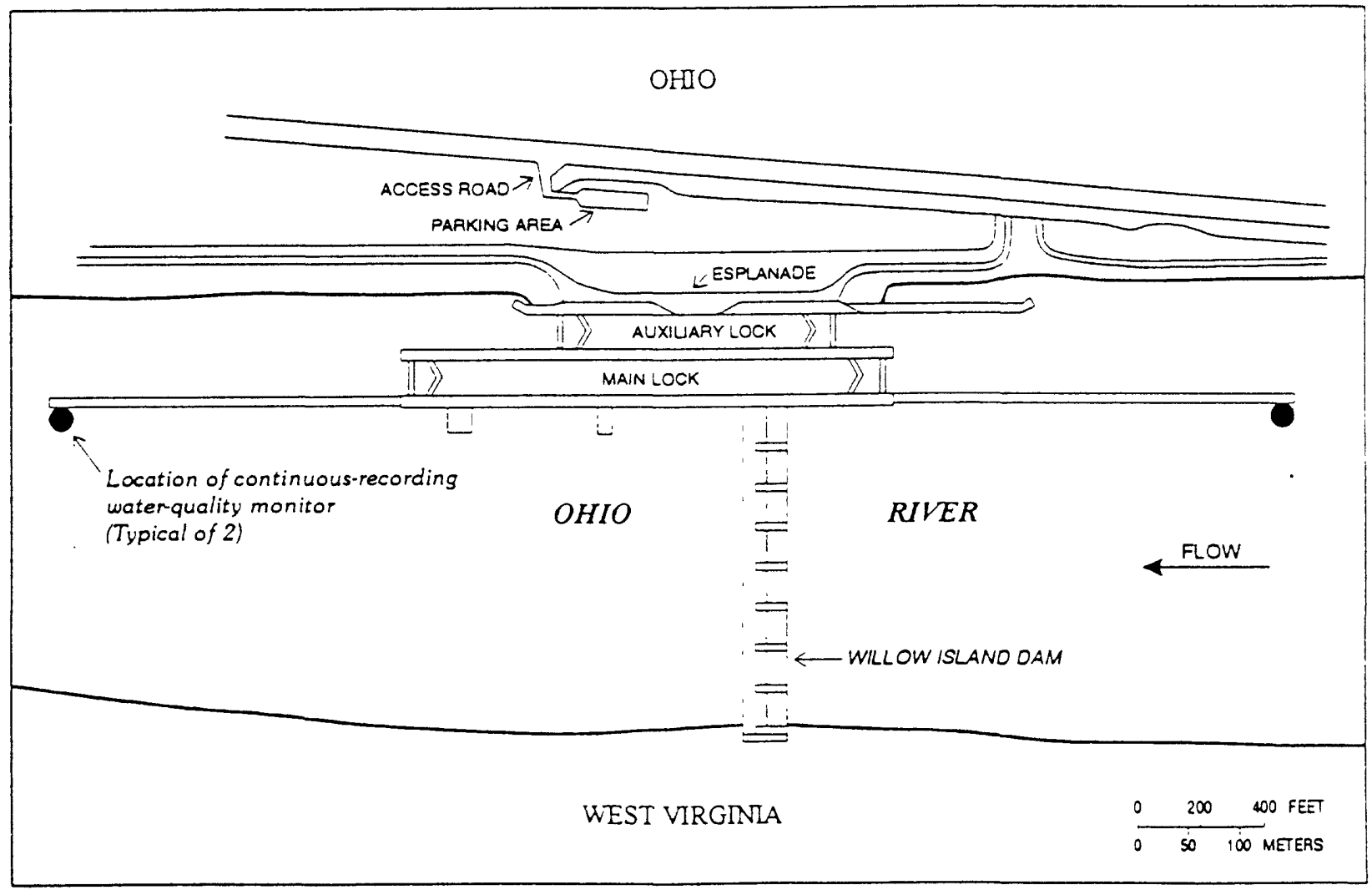

Figure 4. Diagram of Willow Island Dam showing locations of continuous-recording water-quality monitors. 
concentration measurements, the electrode response was tested with a solution of sodium sulfite of sufficient concentration (about $1 \mathrm{~g} / \mathrm{L}$ ) to reduce $\mathrm{DO}$ concentration to below the detection limit $(0.2 \mathrm{mg} / \mathrm{L})$ of the meter (Skougstad and others, 1979).

At least once during each set of crosssectional transect measurements, a water sample was collected from a point in the cross section at the same time that electrode measurements were recorded, and the DO concentration of the water sample was determined by the Winkler method with azide modification (American Public Health Association and others, 1992, p. 4-100). The meter response was considered accurate if it differed from the results of the Winkler test by no more than $0.2 \mathrm{mg} / \mathrm{L}$. Differences of less than $0.2 \mathrm{mg} / \mathrm{L}$ in reported DO concentrations probably are not significant. DO concentration as a percentage of the saturation concentration was calculated using the equations and tables of Weiss (1970).

Secchi disk measurements were made by the same individual between the hours of 1000 and 1600 EDT. Secchi disk depths were not recorded if the sampling time was outside this time window or if high flows or surface waves made measuring impossible.

The continuous-recording water-quality monitors were serviced and recalibrated according to the manufacturer's instructions at least once every 2 weeks, and more frequently during periods of high water temperatures and low river flows. Two sensor packages were available for each monitoring location so that a precalibrated unit could be installed at a site and the existing unit removed and returned to the laboratory for servicing. Data were transmitted from the Data Collection Platform (DCP) by way of the GOES satellite to a local read-out ground station and from there by way of Internet to prlme, a minicomputer located in Towson, Md. After being transmitted to prlme, the data were processed through Device Conversion \& Delivery System (DECODES) and loaded into standard data format into the Automated Data Processing System (ADAPS). Occasionally, there were interruptions to the satellite transmissions and the process was altered. Amendments to the process included downloading the data from the DCP to a disk and manually processing it through DECODES. Personnel from the USGS West Virginia District performed daily quality control by scanning the unedited data, and checking for data interruptions and erroneous values. Raw data were checked for meter drift and corrected, when necessary, by assuming a linear rate of change between successive recalibrations.

\section{WATER-QUALITY DATA}

Water-quality data collected in the Ohio River from Willow Island Dam to Belleville Dam during June through October 1995 are presented in tables 1 through 38. Data for the cross-sectional and longitudinal transects are presented in tables 1 through 30 . The data are arranged according to location of sampling, date, and depth of sampling. Summaries of continuously recorded water-quality data are presented in tables 31 through 38 and are arranged according to date, parameter sampled, and location of sample.

\section{Cross-sectional and Longitudinal- Transect Data}

Tables 1 through 30 present water-quality data for cross-sectional and longitudinal transects. Each table contains all water-quality data collected during 1995 at the sampling point indicated. Sampling points are identified by site number (latitude and longitude) and by river mile. The main shipping channel in the Belleville pool is always to the right (the Ohio side) of large islands; the back channel is always to the left (the West Virginia side) of the islands. In this report, locations for both main-channel and back-channel sampling sites are always given as the total distance from the left bank to the middle of the channel. Data are stored electronically in the U.S. Geological Survey Water Data Storage and Retrieval System (WATSTORE). At locations where cross-sectional-transect data were collected, the location of each depth profile is given as the estimated distance in feet from the left bank of the river and the sampling depth is given in feet below the surface of the water. 
Complete sets of field measurements are reported for the June 28, July 13, July 27, August 24 , September 6, September 20, October 5, and October 19 sampling periods. Severe fog on the river precluded near-sunrise sampling during the August 10 sampling period.

Secchi disk transparency is a measure of the relative amount of light available for photosynthesis (Wetzel and Likens, 1979). The depth at which the Secchi disk disappears from view is affected by the concentration of suspended particles and by light-absorbing characteristics of the water. Occasionally, Secchi disk measurements were missing because the sampling time was not between the hours of 1000 and 1600 or because of adverse weather conditions.

\section{Continuous-Recording Monitor Data}

Continuously monitored water-quality data for the Ohio River at the Willow Island Dam monitors from June through October 1995 are summarized in tables 31 through 38 . These tables contain daily maximum, minimum, and mean values for specific conductance, water temperature, and DO concentration, and daily maximum, minimum, and median values for $\mathrm{pH}$, for both upstream and downstream continuous-recording monitors. The location of the monitors are identified by site number and as either the upstream or the downstream location (fig. 4). If less than 80 percent of hourly values were recorded for a day, a mean value was not reported for that day. Hourly records are stored permanently in the USGS National Water Information System (NWIS) data base, and are available from the Charleston, W.Va. district office. 


\section{SUMMARY}

The water-quality data presented in this report were collected during the summer and fall of 1995 as part of a monitoring program designed to assess the effects of hydropower development on water quality in the Belleville navigation pool of the Ohio River (Ohio River miles 160.6 to 203.6). Data-collection methods combined synoptic sampling throughout the pool and continuous monitoring upstream and downstream from Willow Island Dam. The data were collected, in part, to satisfy license requirements (FERC Project No. 6902) for development of hydropower at Willow Island Dam.

Synoptic sampling consisted of collecting measurements of specific conductance, $\mathrm{pH}$, water temperature, and dissolved oxygen (DO) concentration along a longitudinal transect consisting of 24 main-channel sampling sites and 6 sites on the back-channel (West Virginia) sides of Marietta, Muskingum, and Blennerhassett Islands. Longitudinal-transect and back-channel sites were sampled in the middle of the channel at depths of about surface, $3.0 \mathrm{ft}$ below the surface of the water, at the middle of the water column, and near the bottom of the river. Cross-sectional transects of the same water-quality measurements were made at 6 of the 24 main-channel sites. Three of these cross-sectional transects (river miles 183.0, 184.6, and 192.9) had water-quality measurements taken at near-sunrise and during the afternoon of the same day. Cross-sectional transects consisted of three or four vertical profiles with measurements at the surface of the water, about $3.0 \mathrm{ft}$ below the surface, and then at depth intervals of about $5.0 \mathrm{ft}$ to the bottom of the river. An estimate of the depth of light penetration (Secchi disk depth) was made at each cross-section sampling site whenever light and river-surface conditions were appropriate. The entire network was sampled 9 times from June 28 to October 19 , 1995.
As weather permitted, each synoptic sampling period consisted of near-sunrise crosssectional transect measurements of water quality at river miles $183.0,184.6$, and 192.9. These same sampling sites were also measured during the afternoon of the same day of the near-sunrise measurements. An exception to this protocol was the August 10 sampling period, when fog prohibited all near-sunrise water-quality measurements.

Continuous-recording water-quality monitors were installed at the ends of the upstream and downstream wingwalls at Willow Island Dam. Hourly measurements of specific conductance, $\mathrm{pH}$, water temperature, and DO concentration were recorded beginning in June and continued through October 1995. Maximum, minimum, and mean daily values of specific conductance, water temperature, and DO concentration are reported. Maximum, minimum, and median daily values of $\mathrm{pH}$ are reported. At the upstream monitor maximum and minimum measurements for specific conductance were $667 \mathrm{uS} / \mathrm{cm}$ and $260 \mathrm{uS} /$ $\mathrm{cm}$, for $\mathrm{pH}$ were 8.4 and 7.0 , for water temperature were $32.4^{\circ} \mathrm{C}$ and $15.8^{\circ} \mathrm{C}$, and for dissolved oxygen were $11.5 \mathrm{mg} / \mathrm{l}$ and $5.3 \mathrm{mg} /$, respectively . At the downstream monitor maximum and minimum measurements for specific conductance were $675 \mathrm{uS} / \mathrm{cm}$ and $257 \mathrm{uS} / \mathrm{cm}$, for $\mathrm{pH}$ were 8.1 and 7.1 , for water temperature were $31.2^{\circ} \mathrm{C}$ and $16.0^{\circ} \mathrm{C}$, and for dissolved oxygen $10.7 \mathrm{mg} / \mathrm{l}$ and $5.6 \mathrm{mg} / \mathrm{l}$, respectively. 
American Public Health Association, American Water Works Association, and Water Pollution Control Association, 1992, Standard methods for the examination of water and wastewater, 18th ed.:

Washington, D.C., p. 4-100.

Avery, S.T., and Novak, Pavel, 1978, Oxygen transfer at hydraulic structures: Journal of the Hydraulics Division, Proceedings of the American Society of Civil Engineers, v. 104, no. HY11, p. 1521-1540.

Daniil, E.I., Gulliver, J.S., and Thene, J.R., 1991, Water-quality impact assessment for hydropower: Journal of Environmental Engineering, v. 117, no. 2, p. 179-192.

Federal Energy Regulatory Commission, 1988, Hydroelectric development in the upper Ohio River basin--Final environmental impact statement, FERC Docket No. EL85-19-114: Washington, D.C., Federal Energy Regulatory Commission, Office of Hydropower Licensing [variously paged].

Gulliver, J.S., Daniil, E.I., and Thene, J.R., 1990, Assessing hydro projects' effect on DO concentration: Hydro-Review, v. 9 , no. 6 , p. 62-69.

Hydrolab Corporation, 1991, Surveyor 3. Multiparameter water quality logging system. Operating manual: Austin, Tex., Hydrolab Corporation, $87 \mathrm{p}$.
Ohio River Valley Water Sanitation Commission, 1988, Ohio River water quality fact book 1988: Cincinnati, Ohio, Ohio River Valley Water Sanitation Commission, 157 p.

Skougstad, M.W., and others, 1979, Methods for determination of inorganic substances in water and fluvial sediments: U.S. Geological Survey Techniques of Water-Resources Investigations, book 5, chap. A 1, p. 537-544.

U.S. Army Corps of Engineers, 1990, Ohio River navigation system, 1990: Cincinnati, Ohio, U.S. Army Corps of Engineers, Ohio River Division, $55 \mathrm{p}$.

Weiss, R.F., 1970, The solubility of nitrogen, oxygen and argon in water and seawater: Deep Sea Research, v. 17, no. 4, p. 721-735.

West Virginia Department of Natural Resources, 1988, Ohio River basin plan: Charleston, W. Va., Department of Natural Resources. $350 \mathrm{p}$. 1989, West Virginia water quality status assessment, 1987-1989: Charleston, W. Va., West Virginia Department of Natural Resources, $131 \mathrm{p}$.

Wetzel, R.G., and Likens, G.E., 1979, Limnological analysis: Philadelphia, W.B. Saunders Company, 357 p. 


\section{Tables 1-30 Headnote explanation}

At each longitudinal-transect and back-channel sampling site, measurements of specific conductance, $\mathrm{pH}$, water temperature, and dissolved oxygen concentration were made at four depths (surface of the water, about 3.0 feet below the surface, middle of the water column, and near the bottom of the river) constituting a four-point vertical profile. Cross-sectional transects consisted of three or four detailed vertical profiles of the same characteristics. Estimates of the depth of light penetration (Secchi disk transparency) were made at cross-sectional sampling locations whenever light and river-surface conditions were appropriate. Each synoptic sampling period was completed in two days or less. The entire network was sampled nine times from June 28 to October 19, 1995. 
Table 1. Water-quality data for station 392211081181201, Ohio River at river mile 160.6, June to October, 1995.

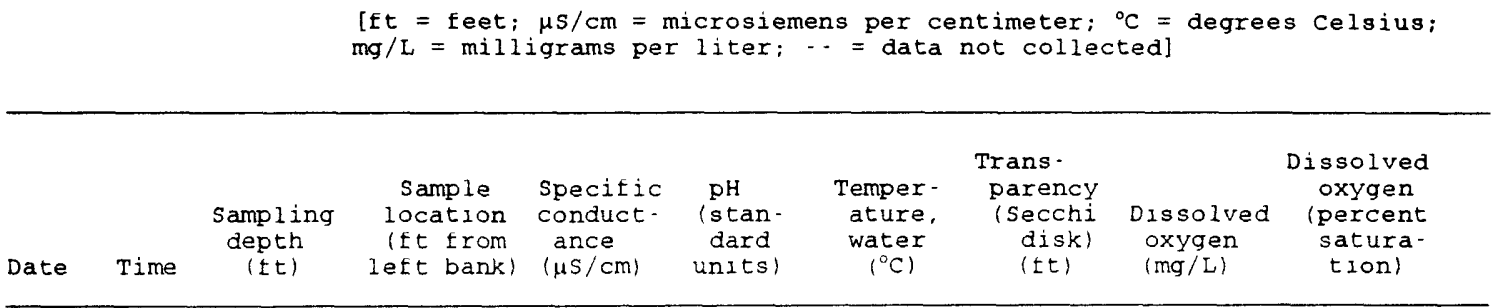

June

\begin{tabular}{|c|c|c|c|c|c|c|c|c|}
\hline 0955 & 0.3 & 700 & 349 & 7.3 & 27.3 & -- & 6.9 & 89 \\
\hline 0956 & 3.0 & 700 & 350 & 7.3 & 27.3 & $\cdots$ & 6.8 & 88 \\
\hline 0957 & 12 & 700 & 350 & 7.2 & 27.2 & $\cdots$ & 6.6 & 84 \\
\hline 0956 & & & & 72 & 372 & . & 66 & \\
\hline
\end{tabular}

29

$0956 \quad 24$

$\begin{array}{ll}700 & 347\end{array}$

$7.2 \quad 27.2$

6.6

84

Ju1y

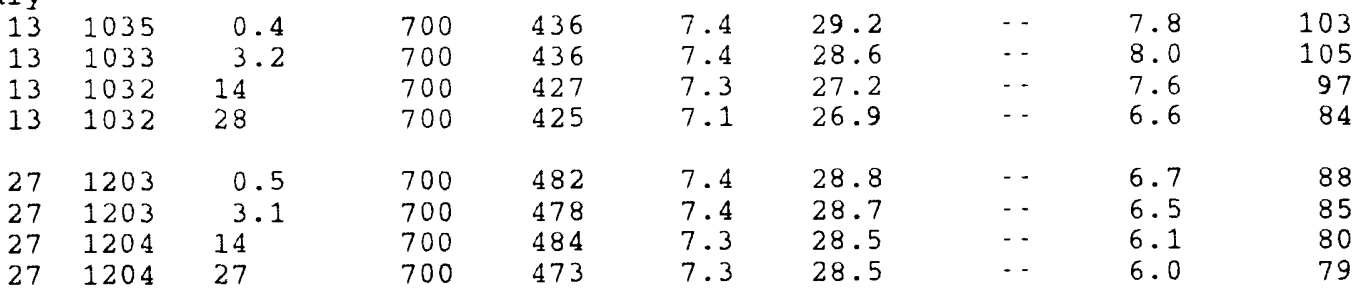

August

\begin{tabular}{|c|c|c|c|c|c|c|c|c|c|}
\hline $9 u=$ & & & & & & & & & \\
\hline 10 & 1147 & 0.4 & 700 & 473 & 7.5 & 30.5 & $\cdots$ & 6.3 & 86 \\
\hline 10 & 1148 & 3.1 & 700 & 474 & 7.5 & 30.2 & $\cdots$ & 6.3 & 85 \\
\hline 10 & 1149 & 12 & 700 & 472 & 7.4 & 29.3 & $\cdots$ & 6.1 & 82 \\
\hline 10 & 1148 & 25 & 700 & 473 & 7.4 & 29.0 & $\cdots$ & 5.9 & \\
\hline 24 & 1847 & 0.5 & 700 & 523 & 7.3 & 31.6 & $\cdots$ & 6.6 & \\
\hline 24 & 1848 & 3.3 & 700 & 525 & 7.3 & 31.3 & $\cdots$ & 6.6 & \\
\hline 24 & 1849 & 14 & 700 & 519 & 7.3 & 30.0 & $\cdots$ & 6.7 & \\
\hline 24 & 1848 & 27 & 700 & 516 & 7.3 & 29.5 & $\cdots$ & 6.0 & \\
\hline$p t$ & ber & & & & & & & & \\
\hline 06 & 0944 & 0.3 & 700 & 580 & 7.2 & 29.4 & $\cdots$ & 7.2 & \\
\hline 06 & 0944 & 3.3 & 700 & 580 & 7.2 & 29.2 & $\cdots$ & 7.2 & \\
\hline 06 & 0946 & 14 & 700 & 580 & 7.2 & 27.6 & $\cdots$ & 6.7 & \\
\hline 06 & 0945 & 28 & 700 & 577 & 7.2 & 27.5 & - & 6.6 & \\
\hline 19 & 1348 & 0.3 & 700 & 645 & $7 \cdot 3$ & 24.9 & $\cdots$ & 6.4 & \\
\hline 19 & 1349 & 3.1 & 700 & 645 & $7 \cdot 3$ & 24.7 & $\cdots$ & 6.2 & \\
\hline 19 & 1350 & 13 & 700 & 642 & 7.3 & 24.1 & $\cdots$ & 6.1 & \\
\hline 19 & 1349 & 26 & 700 & 639 & 7.3 & 24.1 & $\cdots$ & 6.0 & \\
\hline
\end{tabular}


Table 1. Water-quality data for station 392211081181201, Ohio River at river mile 160.6, June to October, 1995, Continued.

\begin{tabular}{|c|c|c|c|c|c|c|c|c|c|}
\hline Date & Time & $\begin{array}{l}\text { Sampling } \\
\text { depth } \\
\text { (ft) }\end{array}$ & $\begin{array}{l}\text { Sample } \\
\text { location } \\
\text { (ft from } \\
\text { lett bank) }\end{array}$ & $\begin{array}{l}\text { Specific } \\
\text { conduct- } \\
\text { ance } \\
(\mu \mathrm{S} / \mathrm{cm})\end{array}$ & $\begin{array}{l}\mathrm{pH} \\
\text { (stan- } \\
\text { dard } \\
\text { units) }\end{array}$ & $\begin{array}{c}\text { Temper- } \\
\text { ature, } \\
\text { water } \\
\left({ }^{\circ} \mathrm{C}\right)\end{array}$ & $\begin{array}{c}\text { Trans- } \\
\text { parency } \\
\text { (Secchl } \\
\text { disk) } \\
\text { (tt) }\end{array}$ & $\begin{array}{l}\text { Dissolved } \\
\text { oxygen } \\
(\mathrm{mg} / \mathrm{L})\end{array}$ & $\begin{array}{c}\text { Dissolved } \\
\text { oxygen } \\
\text { (percent } \\
\text { satura- } \\
\text { tion) }\end{array}$ \\
\hline \multicolumn{10}{|c|}{ September } \\
\hline 21 & 1346 & 0.5 & 700 & 640 & 7.2 & 26.9 & $\cdots$ & 5.7 & 72 \\
\hline 21 & 1346 & 3.1 & 700 & 643 & 7.2 & 25.5 & $\cdots$ & 5.8 & 72 \\
\hline 21 & 1347 & 13 & 700 & 638 & 7.2 & 24.1 & - & 5.7 & 69 \\
\hline 21 & 1347 & 27 & 700 & 632 & 7.2 & 24.0 & $\cdots$ & 5.6 & 67 \\
\hline \multicolumn{10}{|c|}{ October } \\
\hline 05 & 1907 & 0.2 & 700 & 579 & 7.5 & 22.1 & $\cdots$ & 6.8 & 81 \\
\hline 05 & 1909 & 2.9 & 700 & 577 & 7.4 & 22.1 & $\cdots$ & 6.7 & 78 \\
\hline 05 & 1910 & 15 & 700 & 587 & 7.4 & 22.1 & $\cdots$ & 6.7 & 78 \\
\hline 05 & 1911 & 29 & 700 & 584 & 7.4 & 22.1 & $\cdots$ & 6.6 & 79 \\
\hline 19 & 1249 & 0.6 & 700 & 586 & 7.5 & 21.5 & $\cdots$ & 7.6 & 87 \\
\hline 19 & 1249 & 3.2 & 700 & 589 & 7.5 & 21.2 & -- & 7.5 & 86 \\
\hline 19 & 1250 & 14 & 700 & 585 & 7.5 & 19.5 & $\cdots$ & 7.3 & 81 \\
\hline 19 & 1250 & 28 & 700 & 587 & 7.5 & 19.2 & -- & 7.2 & 79 \\
\hline
\end{tabular}


Table 2. Water-quality data for station 392142081185201, Ohio River at river mile 161.4, June to October, 1995.

\begin{tabular}{|c|c|c|c|c|c|c|c|c|c|}
\hline Date & Time & $\begin{array}{l}\text { Sampling } \\
\text { depth } \\
\text { (ft) }\end{array}$ & $\begin{array}{c}\text { Sample } \\
\text { location } \\
\text { (ft Erom } \\
\text { left bank) }\end{array}$ & $\begin{array}{l}\text { Specific } \\
\text { conduct- } \\
\text { ance } \\
(\mu \mathrm{s} / \mathrm{cm})\end{array}$ & $\begin{array}{l}\mathrm{pH} \\
\text { (stan- } \\
\text { dard } \\
\text { units) }\end{array}$ & $\begin{array}{c}\text { Temper- } \\
\text { ature, } \\
\text { water } \\
\left({ }^{\circ} \mathrm{C}\right)\end{array}$ & $\begin{array}{l}\text { Trans- } \\
\text { parency } \\
\text { (Secchi } \\
\text { drsk) } \\
\text { (ft) }\end{array}$ & $\begin{array}{l}\text { Dissolved } \\
\text { oxygen } \\
(\mathrm{mg} / \mathrm{L})\end{array}$ & $\begin{array}{c}\text { Dissolved } \\
\text { oxygen } \\
\text { (percent } \\
\text { satura- } \\
\text { tion) }\end{array}$ \\
\hline \multicolumn{10}{|l|}{ June } \\
\hline 29 & 1003 & 0.3 & 300 & 352 & 7.3 & 27.6 & $\cdots$ & 6.9 & 89 \\
\hline 29 & 1003 & 2.8 & 300 & 352 & 7.3 & 27.6 & - & 6.9 & 89 \\
\hline 29 & 1004 & 4.9 & 300 & 350 & 7.2 & 27.3 & $\cdots$ & 6.6 & 85 \\
\hline 29 & 1005 & 9.6 & 300 & 353 & 7.2 & 27.2 & - & 6.6 & 84 \\
\hline 29 & 1005 & 15 & 300 & 354 & 7.2 & 27.2 & $\cdots$ & 6.6 & 84 \\
\hline 29 & 1006 & 20 & 300 & 352 & 7.2 & 27.2 & $-\cdot$ & 6.6 & 84 \\
\hline 29 & 1007 & 25 & 300 & 349 & 7.2 & 27.2 & -- & 6.6 & 84 \\
\hline 29 & 1011 & 0.3 & 600 & 351 & 7.3 & 27.5 & - - & 6.8 & 88 \\
\hline 29 & 1011 & 2.6 & 600 & 351 & 7.3 & 27.4 & 1.5 & 6.7 & 86 \\
\hline 29 & 1012 & 5.3 & 600 & 353 & 7.2 & 27.2 & - & 6.6 & 85 \\
\hline 29 & 1013 & 10 & 600 & 352 & 7.2 & 27.2 & $\cdots$ & 6.6 & 84 \\
\hline 29 & 1013 & 15 & 600 & 352 & 7.2 & 27.2 & $\cdots$ & 6.6 & 85 \\
\hline 29 & 1014 & 20 & 600 & 352 & 7.2 & 27.2 & 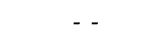 & 6.6 & 84 \\
\hline 29 & 1015 & 25 & 600 & 351 & 7.2 & 27.2 & $\cdots$ & 6.6 & 84 \\
\hline 29 & 1016 & 30 & 600 & 355 & 7.2 & 27.2 & $\cdots$ & 6.6 & 84 \\
\hline 29 & 1020 & 0.3 & 900 & 353 & 7.3 & 27.5 & $\cdots$ & 7.5 & 97 \\
\hline 29 & 1020 & 3.3 & 900 & 352 & 7.3 & 27.4 & $\cdots$ & 6.8 & 88 \\
\hline 29 & 1021 & 5.1 & 900 & 350 & 7.3 & 27.4 & $\cdots$ & 6.6 & 86 \\
\hline 29 & 1021 & 9.9 & 900 & 352 & 7.2 & 27.3 & $\cdots$ & 6.6 & 85 \\
\hline 29 & 1022 & 15 & 900 & 351 & 7.2 & 27.2 & $\cdots$ & 6.6 & 84 \\
\hline 29 & 1022 & 20 & 900 & 351 & 7.2 & 27.2 & $\cdots$ & 6.5 & 84 \\
\hline 29 & 1023 & 25 & 900 & 350 & 7.2 & 27.2 & $\cdots$ & 6.5 & 84 \\
\hline 29 & 1023 & 30 & 900 & 352 & 7.2 & 27.2 & $\cdots$ & 6.5 & 84 \\
\hline 29 & 1028 & 0.3 & 1,300 & 352 & 7.3 & 27.4 & $\cdots$ & 6.8 & 87 \\
\hline 29 & 1028 & 2.9 & 1,300 & 351 & 7.3 & 27.3 & $\cdots$ & 6.7 & 86 \\
\hline 29 & 1029 & 5.1 & 1,300 & 351 & 7.3 & 27.3 & $\cdots$ & 6.7 & 86 \\
\hline 29 & 1029 & 9.8 & 1,300 & 351 & 7.3 & 27.4 & $\cdots$ & 6.7 & 86 \\
\hline 29 & 1030 & 15 & 1,300 & 353 & 7.2 & 27.4 & $\cdots$ & 6.7 & 86 \\
\hline 29 & 1031 & 20 & 1,300 & 351 & 7.2 & 27.4 & - & 6.7 & 86 \\
\hline 29 & 1030 & 25 & 1,300 & 348 & 7.2 & 27.3 & -- & 6.6 & 85 \\
\hline 29 & 1031 & 30 & 1,300 & 352 & 7.3 & 27.3 & $\cdots$ & 6.6 & 85 \\
\hline 29 & 1032 & 33 & 1,300 & 352 & 7.3 & 27.3 & $\cdots$ & 6.6 & 85 \\
\hline
\end{tabular}


Table 2. Water-quality data for station 392142081185201, Ohio River at river mile 161.4, June to October, 1995, Continued.

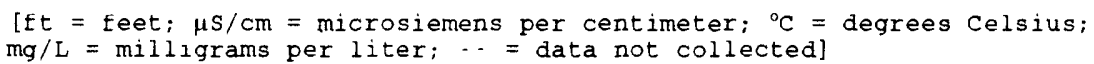

\begin{tabular}{|c|c|c|c|c|c|c|c|c|c|}
\hline Date & Time & $\begin{array}{l}\text { Sampling } \\
\text { depth } \\
\text { (ft) }\end{array}$ & $\begin{array}{c}\text { Sample } \\
\text { location } \\
\text { (tt trom } \\
\text { left bank) }\end{array}$ & $\begin{array}{l}\text { Specific } \\
\text { conduct. } \\
\text { ance } \\
(\mu \mathrm{s} / \mathrm{cm})\end{array}$ & $\begin{array}{l}\mathrm{pH} \\
\text { (stan- } \\
\text { dard } \\
\text { unzts) }\end{array}$ & $\begin{array}{c}\text { Temper- } \\
\text { ature, } \\
\text { water } \\
\left({ }^{\circ} \mathrm{C}\right)\end{array}$ & $\begin{array}{l}\text { Trans - } \\
\text { parency } \\
\text { (Secchi } \\
\text { disk) } \\
\text { (Et) }\end{array}$ & $\begin{array}{c}\text { Dissolved } \\
\text { oxygen } \\
\text { (mg/L) }\end{array}$ & $\begin{array}{c}\text { Dissolved } \\
\text { oxygen } \\
\text { (percent } \\
\text { satura- } \\
\text { tion) }\end{array}$ \\
\hline
\end{tabular}

\begin{tabular}{|c|c|c|c|c|c|c|c|c|c|}
\hline $\begin{array}{r}\text { July } \\
13\end{array}$ & 1044 & 0.7 & 300 & 428 & 7.7 & 28.1 & $\cdots$ & 8.8 & 114 \\
\hline 13 & 1044 & 3.0 & 300 & 427 & 7.7 & 28.0 & $\ldots$ & 8.7 & 113 \\
\hline 13 & 1045 & 5.3 & 300 & 425 & 7.7 & 27.6 & $\ldots$ & 8.7 & 112 \\
\hline 13 & 1045 & 10 & 300 & 424 & 7.6 & 27.5 & - - & 8.3 & 106 \\
\hline 13 & 1046 & 15 & 300 & 425 & 7.4 & 27.2 & - & 7.1 & 91 \\
\hline 13 & 1047 & 20 & 300 & 423 & 7.3 & 27.1 & - & 7.0 & 89 \\
\hline 13 & 1047 & 25 & 300 & 420 & 7.3 & 27.0 & $\cdots$ & 6.6 & 84 \\
\hline 13 & 1048 & 27 & 300 & 424 & 7.3 & 27.0 & $\cdots$ & 6.5 & 83 \\
\hline 13 & 1051 & 0.5 & 600 & 429 & 7.7 & 28.3 & $\cdots$ & 8.7 & 114 \\
\hline 13 & 1051 & 3.4 & 600 & 428 & 7.7 & 28.2 & - & 8.7 & 113 \\
\hline 13 & 1052 & 5.4 & 600 & 424 & 7.6 & 27.6 & -- & 8.4 & 109 \\
\hline 13 & 1053 & 10 & 600 & 425 & 7.5 & 27.5 & -- & 8.0 & 103 \\
\hline 13 & 1053 & 15 & 600 & 424 & 7.4 & 27.3 & $\cdots$ & 7.7 & 99 \\
\hline 13 & 1054 & 20 & 600 & 424 & 7.3 & 27.1 & -- & 7.1 & 90 \\
\hline 13 & 1055 & 25 & 600 & 424 & 7.3 & 27.0 & -- & 6.8 & 87 \\
\hline 13 & 1055 & 30 & 600 & 423 & 7.3 & 27.0 & - & 6.6 & 84 \\
\hline 13 & 1056 & 33 & 600 & 423 & 7.2 & 26.9 & $\cdots$ & 6.6 & 84 \\
\hline 13 & 1059 & 0.6 & 900 & 428 & 7.7 & 28.5 & -- & 8.8 & 116 \\
\hline 13 & 1059 & 3.3 & 900 & 430 & 7.7 & 28.3 & -- & 8.7 & 114 \\
\hline 13 & 1100 & 5.4 & 900 & 428 & 7.7 & 28.3 & $\cdots$ & 8.6 & 112 \\
\hline 13 & 1100 & 10 & 900 & 424 & 7.6 & 27.7 & - & 8.4 & 109 \\
\hline 13 & 1101 & 15 & 900 & 424 & 7.5 & 27.5 & $\cdots$ & 8.1 & 104 \\
\hline 13 & 1101 & 20 & 900 & 424 & 7.4 & 27.3 & $\cdots$ & 7.7 & 99 \\
\hline 13 & 1102 & 25 & 900 & 422 & 7.4 & 27.2 & $\cdots$ & 7.6 & 98 \\
\hline 13 & 1102 & 30 & 900 & 426 & 7.4 & 27.2 & $\cdots$ & 7.4 & 94 \\
\hline 13 & 1103 & 34 & 900 & 427 & 7.3 & 27.1 & $\cdots$ & 7.2 & 91 \\
\hline 13 & 1106 & 0.6 & 1,300 & 430 & 7.8 & 28.5 & $\cdots$ & 8.8 & 115 \\
\hline 13 & 1106 & 3.3 & 1,300 & 427 & 7.8 & 28.3 & -- & 8.7 & 113 \\
\hline 13 & 1107 & 5.2 & 1,300 & 423 & 7.5 & 27.4 & $\cdots$ & 7.8 & 100 \\
\hline 13 & 1107 & 10 & 1,300 & 422 & 7.4 & 27.2 & $\cdots$ & 7.3 & 93 \\
\hline 13 & 1108 & 15 & 1,300 & 422 & 7.4 & 27.2 & - & 7.3 & 93 \\
\hline 13 & 1108 & 20 & 1,300 & 422 & 7.3 & 27.2 & $\cdots$ & 7.2 & 92 \\
\hline 13 & 1109 & 25 & 1,300 & 423 & 7.3 & 27.2 & $\cdots$ & 7.3 & 93 \\
\hline 13 & 1109 & 30 & 1,300 & 423 & 7.3 & 27.2 & $\cdots$ & 7.2 & 9 \\
\hline 13 & 1110 & 34 & 1,300 & 422 & 7.3 & 27.1 & $\cdots$ & 7.1 & 90 \\
\hline
\end{tabular}


Table 2. Water-quality data for station 392142081185201, Ohio River at river mile 161.4, June to October, 1995, Continued.

$\left[\mathrm{Et}=\mathrm{Eeet} ; \mu \mathrm{S} / \mathrm{cm}=\right.$ microsiemens per centimeter $;{ }^{\circ} \mathrm{C}=$ degrees Celsius;

$\mathrm{mg} / \mathrm{L}=$ milligrams per liter $;-$ = data not collected]

\begin{tabular}{|c|c|c|c|c|c|c|c|c|c|}
\hline Date & Time & $\begin{array}{l}\text { Samp I Ing } \\
\text { depth } \\
\text { (tt) }\end{array}$ & $\begin{array}{l}\text { Sample } \\
\text { location } \\
\text { ( } \pm \text { t trom } \\
\text { left bank) }\end{array}$ & $\begin{array}{l}\text { Specific } \\
\text { conduct- } \\
\text { ance } \\
(\mu \mathrm{s} / \mathrm{cm})\end{array}$ & $\begin{array}{l}\mathrm{pH} \\
\text { (stan- } \\
\text { dard } \\
\text { units) }\end{array}$ & $\begin{array}{c}\text { Temper- } \\
\text { ature. } \\
\text { water } \\
\left({ }^{\circ} \mathrm{C}\right)\end{array}$ & $\begin{array}{c}\text { Trans- } \\
\text { parency } \\
\text { (Secch1 } \\
\text { disk) } \\
(t t)\end{array}$ & $\begin{array}{c}\text { Dissolved } \\
\text { oxygen } \\
\text { (mg/L) }\end{array}$ & $\begin{array}{c}\text { Dissolved } \\
\text { oxygen } \\
\text { (percent } \\
\text { satura- } \\
\text { tion) }\end{array}$ \\
\hline
\end{tabular}

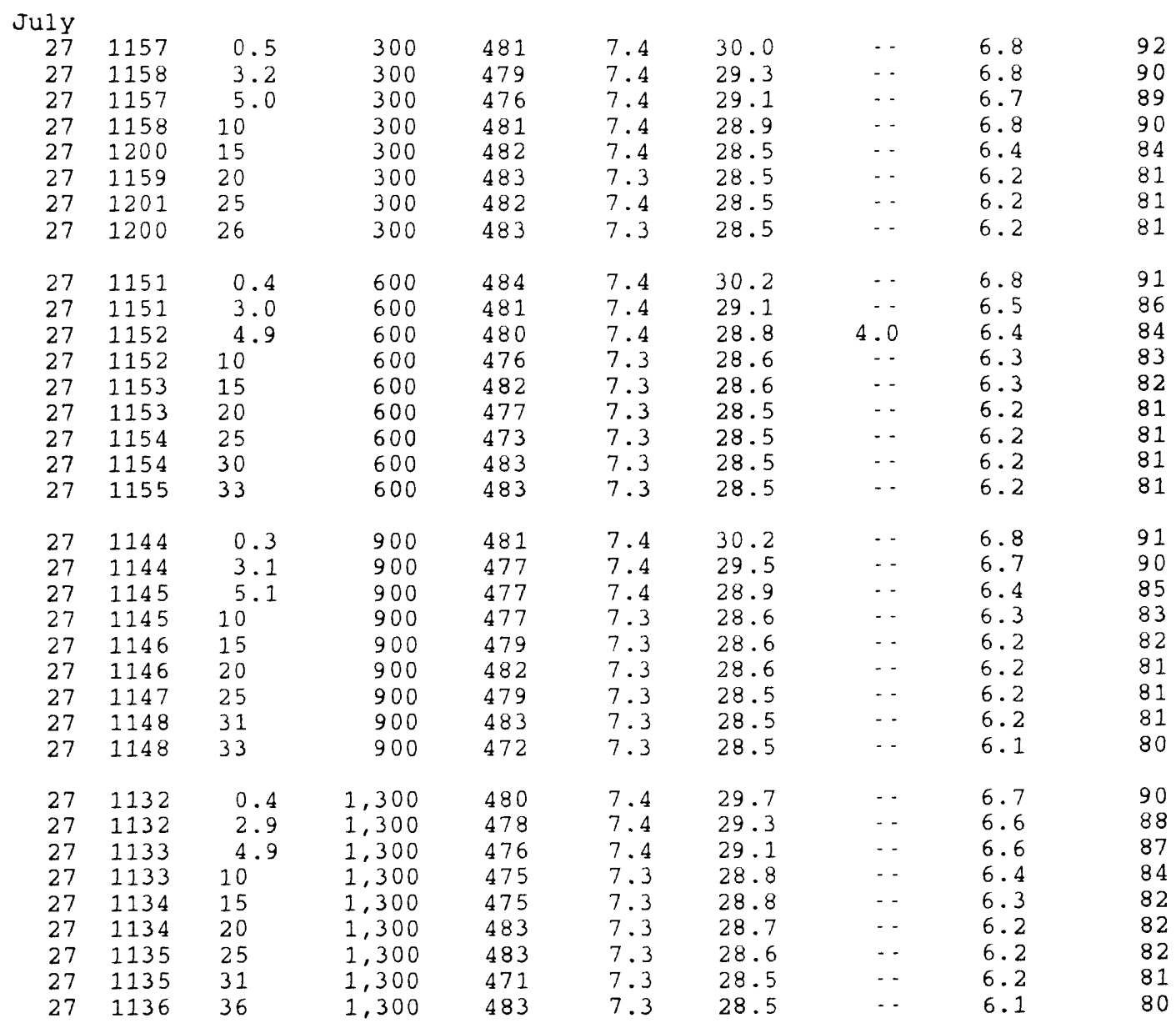


Table 2. Water-quality data for station 392142081185201, Ohio River at river mile 161.4, June to October, 1995, Continued.

$\left[\mathrm{ft}=\mathrm{feet} ; \mu \mathrm{S} / \mathrm{cm}=\mathrm{microsiemens}\right.$ per centimeter $;{ }^{\circ} \mathrm{C}=$ degrees Celsius; $\mathrm{mg} / \mathrm{L}=\mathrm{milligrams}$ per liter: - = data not collected]

\begin{tabular}{|c|c|c|c|c|c|c|c|c|c|}
\hline Date & Time & $\begin{array}{l}\text { Sampling } \\
\text { depth } \\
\text { (ft) }\end{array}$ & $\begin{array}{c}\text { Sample } \\
\text { location } \\
\text { (ft from } \\
\text { left bank) }\end{array}$ & $\begin{array}{l}\text { Specific } \\
\text { conduct- } \\
\text { ance } \\
(\mu \mathrm{S} / \mathrm{cm})\end{array}$ & $\begin{array}{l}\mathrm{pH} \\
\text { (stan- } \\
\text { dard } \\
\text { unlts) }\end{array}$ & $\begin{array}{c}\text { Temper- } \\
\text { ature, } \\
\text { water } \\
\left({ }^{\circ} \mathrm{C}\right)\end{array}$ & $\begin{array}{l}\text { Trans- } \\
\text { parency } \\
\text { (Secchl } \\
\text { disk) } \\
\text { (Et) }\end{array}$ & $\begin{array}{c}\text { Dissolved } \\
\text { oxygen } \\
\text { (mg/L) }\end{array}$ & $\begin{array}{c}\text { Dissolved } \\
\text { oxygen } \\
\text { (percent } \\
\text { satura- } \\
\text { tion) }\end{array}$ \\
\hline
\end{tabular}

\begin{tabular}{|c|c|c|c|c|c|c|c|c|c|}
\hline Augus & & & & & & & & & \\
\hline 10 & 1142 & 0.3 & 300 & 476 & 7.6 & 30.4 & $\cdots$ & 6.8 & 93 \\
\hline 10 & 1142 & 3.0 & 300 & 475 & 7.5 & 30.0 & $\cdots$ & 6.7 & 90 \\
\hline 10 & 1143 & 5.0 & 300 & 472 & 7.5 & 29.5 & $\cdots$ & 6.6 & 88 \\
\hline 10 & 1143 & 9.6 & 300 & 470 & 7.5 & 29.4 & $\cdots$ & 6.3 & 84 \\
\hline 10 & 1144 & 15 & 300 & 482 & 7.5 & 29.3 & $\ldots$ & 6.2 & 83 \\
\hline 10 & 1144 & 20 & 300 & 481 & 7.5 & 29.3 & $\cdots$ & 6.2 & 82 \\
\hline 10 & 1145 & 24 & 300 & 476 & 7.5 & 29.3 & $\cdots$ & 6.2 & 82 \\
\hline 10 & 1137 & 0.5 & 600 & 475 & 7.5 & 30.3 & - & 6.7 & 91 \\
\hline 10 & 1137 & 3.0 & 600 & 474 & 7.5 & 30.0 & 3.0 & 6.7 & 1 \\
\hline 10 & 1138 & 5.1 & 600 & 475 & 7.5 & 29.6 & - & 6.4 & 86 \\
\hline 10 & 1138 & 9.6 & 600 & 475 & 7.5 & 29.3 & $\cdots$ & 6.3 & 84 \\
\hline 10 & 1139 & 15 & 600 & 478 & 7.5 & 29.3 & $\cdots$ & 6.2 & 82 \\
\hline 10 & 1139 & 19 & 600 & 477 & 7.5 & 29.3 & - & 6.2 & 83 \\
\hline 10 & 1140 & 24 & 600 & 478 & 7.5 & 29.3 & $\cdots$ & 6.3 & 83 \\
\hline 10 & 1140 & 29 & 600 & 479 & 7.4 & 29.1 & $\cdots$ & 5.9 & 79 \\
\hline 10 & 1131 & 0.4 & 900 & 476 & 7.5 & 30.2 & $\cdots$ & 6.6 & 89 \\
\hline 10 & 1131 & 3.0 & 900 & 475 & 7.5 & 29.5 & - & 6.5 & 86 \\
\hline 10 & 1132 & 4.9 & 900 & 474 & 7.5 & 29.5 & $\cdots$ & 6.3 & 84 \\
\hline 10 & 1132 & 9.6 & 900 & 475 & 7.4 & 29.3 & $\cdots$ & 6.1 & 82 \\
\hline 10 & 1133 & 15 & 900 & 477 & 7.4 & 29.3 & $\cdots$ & 6.1 & 81 \\
\hline 10 & 1133 & 20 & 900 & 476 & 7.4 & 29.3 & $\cdots$ & 6.1 & 81 \\
\hline 10 & 1134 & 24 & 900 & 477 & 7.4 & 29.2 & $\cdots$ & 6.1 & 81 \\
\hline 10 & 1134 & 29 & 900 & 479 & 7.4 & 29.2 & $\cdots$ & 6.2 & 82 \\
\hline 10 & 1135 & 33 & 900 & 479 & 7.4 & 29.1 & $\cdots$ & 5.9 & 79 \\
\hline 10 & 1123 & 0.2 & 1,300 & 474 & 7.5 & 29.7 & $\cdots$ & 6.6 & 88 \\
\hline 10 & 1123 & 3.0 & 1,300 & 475 & 7.5 & 29.6 & $\cdots$ & 6.4 & 85 \\
\hline 10 & 1124 & 4.9 & 1,300 & 474 & 7.5 & 29.6 & $\cdots$ & 6.4 & 86 \\
\hline 10 & 1124 & 9.5 & 1,300 & 476 & 7.4 & 29.3 & $\cdots$ & 6.2 & 3 \\
\hline 10 & 1125 & 15 & 1,300 & 478 & 7.4 & 29.3 & $\cdots$ & 6.2 & 83 \\
\hline 10 & 1125 & 19 & 1,300 & 480 & 7.4 & 29.2 & $\cdots$ & 6.2 & 82 \\
\hline 10 & 1126 & 24 & 1,300 & 474 & 7.4 & 29.1 & $\cdots$ & 6.0 & 80 \\
\hline 10 & 1126 & 30 & 1,300 & 479 & 7.4 & 29.1 & $\cdots$ & 6.0 & 80 \\
\hline 10 & 1127 & 34 & 1,300 & 476 & 7.4 & 29.1 & $\cdots$ & 5.9 & 78 \\
\hline
\end{tabular}


Table 2. Water-quality data for station 392142081185201, Ohio River at river mile 161.4, June to October, 1995, Continued.

[Et $=$ Eeet $; \mu \mathrm{S} / \mathrm{cm}=$ microsiemens per centimeter $;{ }^{\circ} \mathrm{C}=$ degrees Celsius:

$\mathrm{mg} / \mathrm{L}=$ milligrams per liter; $\cdots$ = data not collected]

\begin{tabular}{|c|c|c|c|c|c|c|c|c|c|}
\hline Date & Time & $\begin{array}{l}\text { Sampling } \\
\text { depth } \\
(f t)\end{array}$ & $\begin{array}{l}\text { Sample } \\
\text { location } \\
\text { (ft from } \\
\text { left bank) }\end{array}$ & $\begin{array}{l}\text { specific } \\
\text { conduct- } \\
\text { ance } \\
(\mu s / \mathrm{cm})\end{array}$ & $\begin{array}{l}\mathrm{pH} \\
\text { (stan- } \\
\text { dard } \\
\text { units) }\end{array}$ & $\begin{array}{c}\text { Temper- } \\
\text { ature, } \\
\text { water } \\
\left({ }^{\circ} \mathrm{C}\right)\end{array}$ & $\begin{array}{c}\text { Trans- } \\
\text { parency } \\
\text { (secchl } \\
\text { disk) } \\
(t t)\end{array}$ & $\begin{array}{l}\text { Dissolved } \\
\text { oxygen } \\
\text { (mg/L) }\end{array}$ & $\begin{array}{c}\text { Dissolved } \\
\text { oxygen } \\
\text { (percent } \\
\text { satura- } \\
\text { tron) }\end{array}$ \\
\hline
\end{tabular}

\begin{tabular}{|c|c|c|c|c|c|c|c|c|c|}
\hline \multicolumn{10}{|c|}{ August } \\
\hline 24 & 1839 & 0.3 & 300 & 523 & 7.8 & 31.4 & $\cdots$ & 8.3 & 114 \\
\hline 24 & 1840 & 3.3 & 300 & 521 & 7.6 & 31.1 & $\cdots$ & 7.5 & 102 \\
\hline 24 & 1840 & 5.0 & 300 & 523 & 7.6 & 31.1 & $\cdots$ & 6.9 & 95 \\
\hline 24 & 1841 & 9.8 & 300 & 522 & 7.3 & 29.9 & $\cdots$ & 6.0 & 81 \\
\hline 24 & 1842 & 15 & 300 & 522 & 7.3 & 29.8 & $\cdots$ & 6.1 & 81 \\
\hline 24 & 1843 & 20 & 300 & 525 & 7.3 & 29.7 & $\cdots$ & 5.9 & 79 \\
\hline 24 & 1844 & 22 & 300 & 518 & 7.3 & 29.7 & $\cdots$ & 5.9 & 78 \\
\hline 24 & 1831 & 0.5 & 600 & 521 & 7.7 & 31.3 & $\cdots$ & 8.0 & 110 \\
\hline 24 & 1831 & 3.1 & 600 & 526 & 7.7 & 31.4 & - & 7.9 & 109 \\
\hline 24 & 1832 & 5.0 & 600 & 525 & 7.5 & 30.7 & $\cdots$ & 6.9 & 93 \\
\hline 24 & 1832 & 10 & 600 & 523 & 7.3 & 29.9 & - & 6.2 & 83 \\
\hline 24 & 1833 & 15 & 600 & 516 & 7.3 & 29.8 & $\cdots$ & 5.9 & 8 \\
\hline 24 & 1833 & 20 & 600 & 515 & 7.3 & 29.7 & $\cdots$ & 6.0 & 80 \\
\hline 24 & 1834 & 25 & 600 & 523 & 7.3 & 29.6 & - & 5.9 & 9 \\
\hline 24 & 1834 & 30 & 600 & 514 & 7.2 & 29.5 & - & 5.8 & 77 \\
\hline 24 & 1835 & 31 & 600 & 519 & 7.2 & 29.5 & $\cdots$ & 5.7 & 76 \\
\hline 24 & 1824 & 0.3 & 900 & 514 & 7.8 & 31.3 & - & 8.1 & 111 \\
\hline 24 & 1824 & 3.2 & 900 & 523 & 7.5 & 30.8 & $\cdots$ & 7.2 & 98 \\
\hline 24 & 1825 & 5.2 & 900 & 522 & 7.4 & 30.4 & $\cdots$ & 6.5 & 88 \\
\hline 24 & 1825 & 9.8 & 900 & 522 & 7.3 & 30.0 & $\cdots$ & 6.1 & 8 \\
\hline 24 & 1826 & 15 & 900 & 521 & 7.3 & 29.9 & $\cdots$ & 6.2 & 8 \\
\hline 24 & 1826 & 20 & 900 & 521 & 7.3 & 29.8 & $\cdots$ & 6.1 & 2 \\
\hline 24 & 1827 & 25 & 900 & 520 & 7.3 & 29.6 & $\cdots$ & 5.9 & 7 \\
\hline 24 & 1827 & 30 & 900 & 519 & 7.2 & 29.5 & $\cdots$ & 5.8 & 78 \\
\hline 24 & 1828 & 32 & 900 & 518 & 7.2 & 29.4 & $\cdots$ & 5.5 & 73 \\
\hline 24 & 1810 & 0.9 & 1,300 & 521 & 7.4 & 30.5 & $\cdots$ & 6.9 & 94 \\
\hline 24 & 1810 & 3.2 & 1,300 & 521 & 7.4 & 30.5 & $\cdots$ & 6.7 & 90 \\
\hline 24 & 1811 & 5.1 & 1,300 & 522 & 7.4 & 30.5 & $\cdots$ & 6.6 & 89 \\
\hline 24 & 1811 & 9.8 & 1,300 & 522 & 7.4 & 30.4 & -- & 6.6 & 85 \\
\hline 24 & 1812 & 15 & 1.300 & 521 & 7.3 & 30.0 & $\cdots$ & 6.1 & 8 \\
\hline 24 & 1812 & 20 & 1,300 & 521 & 7.3 & 29.8 & $\cdots$ & 6.0 & 81 \\
\hline 24 & 1813 & 25 & 1,300 & 519 & 7.2 & 29.6 & $\cdots$ & 5.8 & 77 \\
\hline 24 & 1813 & 30 & 1,300 & 518 & 7.2 & 29.5 & $\cdots$ & 5.6 & 7 \\
\hline 24 & 1814 & 34 & 1,300 & 518 & 7.2 & 29.5 & - & 5.5 & 73 \\
\hline
\end{tabular}


Table 2. Water-quality data for station 392142081185201, Ohio River at river mile 161.4, June to October, 1995, Continued.

[ft $=$ feet $; \mu \mathrm{s} / \mathrm{cm}=$ macrosiemens per centimeter $;{ }^{\circ} \mathrm{C}=$ degrees Celsius:

$\mathrm{mg} / \mathrm{L}=\mathrm{mili}$ igrams per liter: $\cdots=$ data not collected]

\begin{tabular}{|c|c|c|c|c|c|c|c|c|c|}
\hline Date & Time & $\begin{array}{l}\text { Sampling } \\
\text { depth } \\
\text { (ft) }\end{array}$ & $\begin{array}{c}\text { Sample } \\
\text { location } \\
\text { (tt from } \\
\text { lett bank) }\end{array}$ & $\begin{array}{l}\text { Specific } \\
\text { conduct- } \\
\text { ance } \\
(\mu \mathrm{s} / \mathrm{cm})\end{array}$ & $\begin{array}{l}\mathrm{pH} \\
\text { (stan- } \\
\text { dard } \\
\text { units) }\end{array}$ & $\begin{array}{c}\text { Temper- } \\
\text { ature, } \\
\text { water } \\
\left({ }^{\circ} \mathrm{C}\right)\end{array}$ & $\begin{array}{c}\text { Trans- } \\
\text { parency } \\
\text { (Secchi } \\
\text { disk) } \\
\text { (ft) }\end{array}$ & $\begin{array}{l}\text { Dissolved } \\
\text { oxygen } \\
\text { (mg/L) }\end{array}$ & $\begin{array}{c}\text { Dissolved } \\
\text { oxygen } \\
\text { (percent } \\
\text { satura- } \\
\text { tion) }\end{array}$ \\
\hline
\end{tabular}

$\begin{array}{llcll}\text { September } & & & \\ 06 & 1030 & 1.1 & 300 & 579 \\ 06 & 1030 & 3.6 & 300 & 580 \\ 06 & 1031 & 5.5 & 300 & 579 \\ 06 & 1031 & 10 & 300 & 579 \\ 06 & 1032 & 15 & 300 & 579 \\ 06 & 1032 & 20 & 300 & 579 \\ 06 & 1033 & 21 & 300 & 578 \\ 06 & 1023 & 0.5 & 600 & 579 \\ 06 & 1023 & 3.4 & 600 & 579 \\ 06 & 1024 & 5.5 & 600 & 578 \\ 06 & 1024 & 10 & 600 & 579 \\ 06 & 1025 & 15 & 600 & 577 \\ 06 & 1025 & 20 & 600 & 577 \\ 06 & 1026 & 25 & 600 & 578 \\ 06 & 1026 & 30 & 600 & 577 \\ 06 & 1027 & 33 & 600 & 577 \\ 06 & 1013 & 1.0 & 900 & 580 \\ 06 & 1013 & 3.3 & 900 & 580 \\ 06 & 1014 & 5.1 & 900 & 579 \\ 06 & 1015 & 10 & 900 & 579 \\ 06 & 1016 & 15 & 900 & 578 \\ 06 & 1016 & 20 & 900 & 579 \\ 06 & 1017 & 25 & 900 & 579 \\ 06 & 1018 & 30 & 900 & 579 \\ 06 & 1019 & 33 & 900 & 579 \\ 06 & 0959 & 0.5 & 1,300 & 577 \\ 06 & 0959 & 2.9 & 1,300 & 577 \\ 06 & 1000 & 4.9 & 1,300 & 577 \\ 06 & 1000 & 10 & 1,300 & 578 \\ 06 & 1001 & 15 & 1,300 & 579 \\ 06 & 1001 & 20 & 1,300 & 580 \\ 06 & 1002 & 25 & 1.300 & 577 \\ 06 & 1002 & 30 & 1.300 & 577 \\ 06 & 1003 & 35 & 1,300 & 580 \\ 06 & 1003 & 38 & 1,300 & 580\end{array}$

\begin{tabular}{|c|c|}
\hline 7.3 & 28.3 \\
\hline 7.3 & 28.3 \\
\hline 7.3 & 28.2 \\
\hline 7.2 & 28.0 \\
\hline 7.2 & 28.0 \\
\hline 7.3 & 27.9 \\
\hline 7.3 & 27.9 \\
\hline 7.3 & 28.3 \\
\hline 7.3 & 28.2 \\
\hline 7.2 & 28.1 \\
\hline 7.2 & 28.0 \\
\hline 7. & 28.0 \\
\hline 7. & 28.0 \\
\hline 7 . & 27.9 \\
\hline 7. & 27.9 \\
\hline 7.1 & 27.9 \\
\hline 7.3 & 28.2 \\
\hline 7. & 28.2 \\
\hline 7.3 & 28.2 \\
\hline 7.2 & 28.0 \\
\hline 7.2 & 28.0 \\
\hline 7.2 & 28.0 \\
\hline 7 & 28.0 \\
\hline 7.2 & 28.0 \\
\hline 7.2 & 28.0 \\
\hline 7.3 & 28.1 \\
\hline 7.3 & 28.1 \\
\hline 7.3 & 28.1 \\
\hline 7.3 & 28.1 \\
\hline 7.3 & 28.1 \\
\hline 7.2 & 28.1 \\
\hline 7.3 & 28.1 \\
\hline 7.2 & 28.1 \\
\hline 7 & $\begin{array}{l}28.0 \\
28.0\end{array}$ \\
\hline
\end{tabular}

$-7.4$

$7 \cdot 4$

7.2

6.8

- 6.8

- 7.0

- 6.9

7.2

$\begin{array}{ll}\cdots & 7.2 \\ \cdots & 6.8\end{array}$

$4.0 \quad 6.6$

-. 6.7

- 6.5

6.5

6.4

- 6.2

.. 6.0

$\begin{array}{ll}\text { - } & 7.2 \\ \text { - } & 7.3\end{array}$

- 7.1

- 6.8

. 6.8

6.7

-. 6.8

- 6.6

- 6.6

97

- 7.1

. 7.1

- 7.1

. 7.0

- 6.9

- 6.9

- 7.0

- 7.0

- 6.9

$\begin{array}{lllll}7.3 & 28.0 & \ldots & 6.9 & 89 \\ 7.2 & 28.0 & \ldots & 6.9 & 89\end{array}$

93

88

87
90

88

93

88

86

86

84

84

83

80

93

95

92

88

87

87

88

86

86

92

91

92

90

90

89

90

90
91 
Table 2. Water-quality data for station 392142081185201 , ohio River at river mile 161.4, June to october, 1995, Continued.

$\left[E t=\right.$ feet; $\mu \mathrm{s} / \mathrm{cm}=$ microsiemens per centimeter $;{ }^{\circ} \mathrm{C}=$ degrees Celsius:

$\mathrm{mg} / \mathrm{L}=$ milligrams per liter; .. = data not collected]

\begin{tabular}{|c|c|c|c|c|c|c|c|c|c|}
\hline Date & Time & $\begin{array}{l}\text { Sampling } \\
\text { depth } \\
\text { (ft) }\end{array}$ & $\begin{array}{c}\text { Sample } \\
\text { location } \\
\text { (ft from } \\
\text { left bank) }\end{array}$ & $\begin{array}{c}\text { Specific } \\
\text { conduct- } \\
\text { ance } \\
(\mu \mathrm{s} / \mathrm{cm})\end{array}$ & $\begin{array}{l}\mathrm{pH} \\
\text { (stan- } \\
\text { dard } \\
\text { units) }\end{array}$ & $\begin{array}{c}\text { Temper- } \\
\text { ature, } \\
\text { water } \\
\left({ }^{\circ} \mathrm{C}\right)\end{array}$ & $\begin{array}{c}\text { Trans - } \\
\text { parency } \\
\text { (Secchl } \\
\text { disk) } \\
\text { (Et) }\end{array}$ & $\begin{array}{c}\text { Dissolved } \\
\text { oxygen } \\
\text { (mg } / \mathrm{L}\rangle\end{array}$ & $\begin{array}{c}\text { Dissolved } \\
\text { oxygen } \\
\text { (percent } \\
\text { satura- } \\
\text { tion) }\end{array}$ \\
\hline
\end{tabular}

September

\begin{tabular}{|c|c|c|c|c|c|c|c|c|}
\hline $19 \quad 1341$ & 0.9 & 300 & 643 & 7.3 & 24.4 & $\cdots$ & 6.3 & 76 \\
\hline $19 \quad 1341$ & 2.9 & 300 & 641 & 7.3 & 24.5 & $\cdots$ & 6.4 & 78 \\
\hline 1342 & 4.8 & 300 & 641 & 7.3 & 24.3 & $\cdots$ & 6.4 & 77 \\
\hline 1342 & 9.5 & 300 & 640 & 7.3 & 24.1 & -- & 6.2 & 74 \\
\hline 1343 & 15 & 300 & 647 & 7.2 & 24.1 & $\cdots$ & 6.0 & 73 \\
\hline 1343 & 20 & 300 & 646 & 7.2 & 24.1 & - & 6.1 & 73 \\
\hline 1344 & 25 & 300 & 643 & 7.2 & 24.1 & -- & 6.1 & 73 \\
\hline 1344 & 27 & 300 & 647 & 7.2 & 24.0 & $\cdots$ & 6.0 & 72 \\
\hline 1335 & 1.0 & 600 & 642 & 7.3 & 24.8 & $\cdots$ & 6.6 & 80 \\
\hline 1335 & 2.9 & 600 & 640 & $7 \cdot 3$ & 24.6 & - & 6.3 & 77 \\
\hline 1336 & 4.9 & 600 & 642 & 7.3 & 24.3 & 4.0 & 6.3 & \\
\hline 1337 & 9.7 & 600 & 646 & 7.3 & 24.1 & - & 6.1 & 73 \\
\hline 1337 & 15 & 600 & 646 & 7.2 & 24.1 & - & 6.0 & 72 \\
\hline 1338 & 20 & 600 & 642 & 7.2 & 24.1 & $\cdots$ & 6.0 & \\
\hline 1338 & 25 & 600 & 643 & 7.2 & 24.0 & $\cdots$ & 6.0 & 72 \\
\hline 1339 & 30 & 600 & 643 & 7.2 & 24.0 & -- & 6.0 & 72 \\
\hline 1339 & 33 & 600 & 649 & 7.2 & 23.9 & $\cdots$ & 5.9 & 71 \\
\hline 1333 & 0.3 & 900 & 643 & $7 \cdot 3$ & 24.8 & -- & 6.3 & \\
\hline 1332 & 2.9 & 900 & 640 & 7.3 & 24.6 & - & 6.2 & 7 \\
\hline 1332 & 4.9 & 900 & 638 & 7.3 & 24.3 & - & 6.2 & \\
\hline 1331 & 9.7 & 900 & 639 & 7.2 & 24.1 & $\cdots$ & 6.2 & 74 \\
\hline 1331 & 15 & 900 & 638 & 7.2 & 24.1 & - & 6.0 & 72 \\
\hline 1330 & 20 & 900 & 644 & 7.2 & 24.1 & -- & 5.9 & 73 \\
\hline 1330 & 25 & 900 & 647 & 7.2 & 24.0 & $\cdots$ & 5.9 & 7 \\
\hline 1329 & 30 & 900 & 644 & 7.2 & 24.0 & $\cdots$ & 6.1 & 7 \\
\hline 1329 & 33 & 900 & 637 & 7.2 & 23.8 & $\cdots$ & 6.0 & \\
\hline 1250 & 0.2 & 1,300 & 641 & $7 \cdot 3$ & 24.6 & $\cdots$ & 6.5 & \\
\hline 1250 & 3.0 & 1,300 & 643 & 7.3 & 24.3 & -- & 6.2 & 7 \\
\hline 1251 & 5.0 & 1,300 & 645 & $7 \cdot 3$ & 24.2 & $\cdots$ & 6.1 & \\
\hline 1251 & 10 & 1,300 & 645 & 7.3 & 24.1 & $\cdots$ & 6.0 & 7 \\
\hline 1252 & 15 & 1,300 & 645 & 7.2 & 24.0 & $\cdots$ & 6.0 & 7 \\
\hline 1252 & 20 & 1,300 & 643 & 7.2 & 24.0 & $\cdots$ & 6.0 & \\
\hline 1253 & 25 & 1,300 & 650 & 7.2 & 24.0 & - & 6.0 & 7 \\
\hline 1253 & 30 & 1,300 & 649 & 7.2 & 23.9 & $\cdots$ & 6.0 & \\
\hline 1254 & 35 & 1,300 & 650 & 7.3 & 23.9 & - & 5.9 & \\
\hline
\end{tabular}


Table 2. Water-quality data for station 392142081185201, Ohio River at river mile 161.4, June to October, 1995, Continued.

[ft $=$ feet; $\mu \mathrm{s} / \mathrm{cm}=$ microsiemens per centimeter $;{ }^{\circ} \mathrm{C}=$ degrees Celsius; $\mathrm{mg} / \mathrm{L}=\mathrm{mili}$ grams per liter; $\cdots$ = data not collected]

\begin{tabular}{|c|c|c|c|c|c|c|c|c|c|}
\hline Date & Time & $\begin{array}{l}\text { Sampling } \\
\text { depth } \\
\text { (ft) }\end{array}$ & $\begin{array}{c}\text { Sample } \\
\text { location } \\
\text { (tt trom } \\
\text { left bank) }\end{array}$ & $\begin{array}{l}\text { Specitic } \\
\text { conduct- } \\
\text { ance } \\
(\mu \mathrm{s} / \mathrm{cm})\end{array}$ & $\begin{array}{l}\mathrm{pH} \\
\text { (stan- } \\
\text { dard } \\
\text { units) }\end{array}$ & $\begin{array}{c}\text { Temper- } \\
\text { ature, } \\
\text { water } \\
\left({ }^{\circ} \mathrm{C}\right)\end{array}$ & $\begin{array}{l}\text { Trans - } \\
\text { parency } \\
\text { (Secchi } \\
\text { disk) } \\
\text { (Et) }\end{array}$ & $\begin{array}{l}\text { Dissolved } \\
\text { oxygen } \\
(\mathrm{mg} / \mathrm{L})\end{array}$ & $\begin{array}{c}\text { Dissolved } \\
\text { oxygen } \\
\text { (percent } \\
\text { satura- } \\
\text { tion) }\end{array}$ \\
\hline
\end{tabular}

September

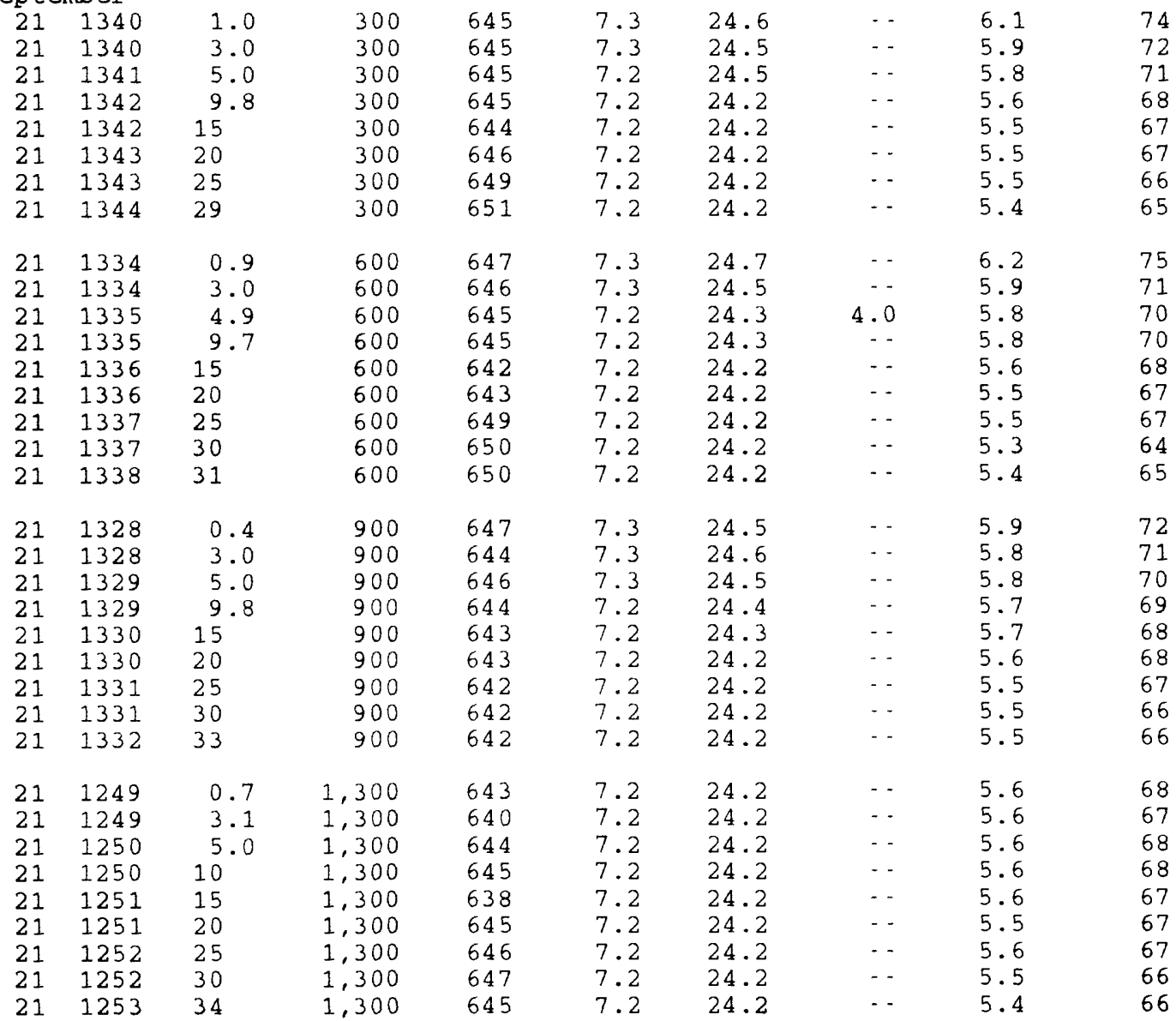


Table 2. Water-quality data for station 392142081185201, Ohio River at river mile 161.4, June to October, 1995, Continued.

\begin{tabular}{|c|c|c|c|c|c|c|c|c|c|}
\hline Date & Time & $\begin{array}{l}\text { Sampling } \\
\text { depth } \\
\text { (ft) }\end{array}$ & $\begin{array}{c}\text { Sample } \\
\text { location } \\
\text { (ft from } \\
\text { left bank) }\end{array}$ & $\begin{array}{l}\text { Specific } \\
\text { conduct- } \\
\text { ance } \\
(\mu \mathrm{S} / \mathrm{cm})\end{array}$ & $\begin{array}{l}\mathrm{pH} \\
\text { (stan- } \\
\text { dard } \\
\text { undts) }\end{array}$ & $\begin{array}{c}\text { Temper- } \\
\text { ature, } \\
\text { water } \\
\left({ }^{\circ} \mathrm{C}\right)\end{array}$ & $\begin{array}{l}\text { Trans- } \\
\text { parency } \\
\text { (Secch } \\
\text { disk) } \\
( \pm t)\end{array}$ & $\begin{array}{l}\text { Dissolved } \\
\text { oxygen } \\
\text { (mg/L) }\end{array}$ & $\begin{array}{r}\text { Dissolv } \\
\text { oxyge } \\
\text { (perce } \\
\text { satur } \\
\text { tron) }\end{array}$ \\
\hline \multicolumn{10}{|c|}{ October } \\
\hline 05 & 1901 & 1.1 & 300 & 571 & 7.4 & 22.1 & $\cdots$ & 6.8 & 79 \\
\hline 05 & 1901 & 3.2 & 300 & 573 & 7.4 & 22.1 & -- & 6.7 & 78 \\
\hline 05 & 1902 & 4.8 & 300 & 578 & 7.4 & 22.1 & -- & 6.7 & 78 \\
\hline 05 & 1903 & 9.8 & 300 & 579 & 7.4 & 22.1 & $\cdots$ & 6.7 & 78 \\
\hline 05 & 1903 & 15 & 300 & 583 & 7.4 & 22.1 & -- & 6.7 & 78 \\
\hline 05 & 1904 & 20 & 300 & 584 & 7.4 & 22.1 & $\cdots$ & 6.6 & 77 \\
\hline 05 & 1904 & 25 & 300 & 589 & 7.4 & 22.1 & $\cdots$ & 6.6 & 77 \\
\hline 05 & 1905 & 29 & 300 & 584 & 7.4 & 22.1 & $\cdots$ & 6.6 & 77 \\
\hline 05 & 1855 & 0.8 & 600 & 573 & 7.4 & 22.1 & $\cdots$ & 6.7 & 78 \\
\hline 05 & 1855 & 3.3 & 600 & 575 & 7.4 & 22.1 & -- & 6.7 & 78 \\
\hline 05 & 1856 & 5.1 & 600 & 577 & 7.4 & 22.1 & $\cdots$ & 6.7 & 78 \\
\hline 05 & 1856 & 9.8 & 600 & 580 & 7.4 & 22.1 & -- & 6.6 & 77 \\
\hline 05 & 1857 & 15 & 600 & 581 & 7.4 & 22.1 & $\cdots$ & 6.6 & 77 \\
\hline 05 & 1857 & 19 & 600 & 582 & 7.4 & 22.1 & $\cdots$ & 6.6 & 77 \\
\hline 05 & 1858 & 25 & 600 & 586 & 7.4 & 22.1 & $\cdots$ & 6.6 & 77 \\
\hline 05 & 1858 & 30 & 600 & 585 & 7.4 & 22.1 & -- & 6.6 & 77 \\
\hline 05 & 1859 & 33 & 600 & 587 & 7.4 & 22.1 & -- & 6.6 & 77 \\
\hline 05 & 1849 & 0.8 & 900 & 570 & 7.5 & 22.1 & $\cdots$ & 6.7 & 78 \\
\hline 05 & 1849 & 3.2 & 900 & 571 & 7.4 & 22.1 & $\cdots$ & 6.7 & 78 \\
\hline 05 & 1850 & 5.2 & 900 & 575 & 7.4 & 22.1 & $\cdots$ & 6.7 & 78 \\
\hline 05 & 1850 & 10 & 900 & 583 & 7.4 & 22.1 & $\cdots$ & 6.6 & 77 \\
\hline 05 & 1851 & 15 & 900 & 579 & 7.4 & 22.1 & $\cdots$ & 6.6 & 77 \\
\hline 05 & 1851 & 20 & 900 & 588 & 7.4 & 22.1 & $\cdots$ & 6.5 & 76 \\
\hline 05 & 1852 & 25 & 900 & 588 & 7.4 & 22.1 & $\cdots$ & 6.5 & 76 \\
\hline 05 & 1852 & 30 & 900 & 578 & 7.4 & 22.1 & $\cdots$ & 6.5 & 76 \\
\hline 05 & 1853 & 34 & 900 & 580 & 7.4 & 22.1 & $\cdots$ & 6.6 & 77 \\
\hline 05 & 1840 & 1.2 & 1,300 & 571 & 7.4 & 22.1 & $\cdots$ & 6.6 & 77 \\
\hline 05 & 1840 & 3.1 & 1,300 & 573 & 7.4 & 22.1 & - & 6.6 & 77 \\
\hline 05 & 1841 & 5.0 & 1,300 & 572 & 7.4 & 22.1 & -- & 6.6 & 77 \\
\hline 05 & 1841 & 10 & 1,300 & 575 & 7.4 & 22.1 & $\cdots$ & 6.6 & 77 \\
\hline 05 & 1842 & 15 & 1,300 & 582 & 7.4 & 22.1 & -- & 6.6 & 77 \\
\hline 05 & 1842 & 20 & 1,300 & 576 & 7.4 & 22.1 & -- & 6.6 & 76 \\
\hline 05 & 1843 & 25 & 1,300 & 581 & 7.4 & 22.1 & $\cdots$ & 6.6 & 76 \\
\hline 05 & 1843 & 30 & 1,300 & 576 & 7.4 & 22.1 & - & 6.5 & 76 \\
\hline 05 & 1844 & 35 & 1,300 & 582 & 7.4 & 22.1 & -- & 6.5 & 75 \\
\hline
\end{tabular}


Table 2. Water-quality data for station 392142081185201, Ohio River at river mile 161.4, June to October, 1995, Continued.

\begin{tabular}{|c|c|c|c|c|c|c|c|c|c|}
\hline Date & Time & $\begin{array}{l}\text { Sampling } \\
\text { depth } \\
(t t)\end{array}$ & $\begin{array}{c}\text { Sample } \\
\text { location } \\
\text { (tt trom } \\
\text { lett bank) }\end{array}$ & $\begin{array}{l}\text { Specitic } \\
\text { conduct- } \\
\text { ance } \\
(\mu \mathrm{S} / \mathrm{cm})\end{array}$ & $\begin{array}{l}\text { pH } \\
\text { (stan- } \\
\text { dard } \\
\text { undts) }\end{array}$ & $\begin{array}{c}\text { Temper- } \\
\text { ature, } \\
\text { water } \\
\left({ }^{\circ} \mathrm{C}\right)\end{array}$ & $\begin{array}{l}\text { Trans - } \\
\text { parency } \\
\text { (Secchi } \\
\text { disk) } \\
\text { (tt) }\end{array}$ & $\begin{array}{l}\text { Dissolved } \\
\text { oxygen } \\
(\mathrm{mg} / \mathrm{L})\end{array}$ & $\begin{array}{c}\text { Dissolved } \\
\text { oxygen } \\
\text { (percent } \\
\text { satura- } \\
\text { tion) }\end{array}$ \\
\hline \multicolumn{10}{|c|}{ October } \\
\hline 19 & 1221 & 1.0 & 300 & 579 & 7.6 & 20.8 & $\cdots$ & 7.6 & 86 \\
\hline 19 & 1222 & 3.2 & 300 & 581 & 7.6 & 20.5 & . & 7.5 & 85 \\
\hline 19 & 1222 & 5.4 & 300 & 577 & 7.6 & 19.9 & $\cdots$ & 7.5 & 83 \\
\hline 19 & 1223 & 10 & 300 & 573 & 7.5 & 19.6 & $\cdots$ & 7.3 & 81 \\
\hline 19 & 1224 & 15 & 300 & 573 & 7.5 & 19.5 & $\cdots$ & 7.3 & 80 \\
\hline 19 & 1224 & 20 & 300 & 572 & 7.5 & 19.5 & $\cdots$ & 7.2 & 80 \\
\hline 19 & 1225 & 23 & 300 & 572 & 7.5 & 19.5 & $\cdots$ & 7.0 & 78 \\
\hline 19 & 1230 & 0.5 & 600 & 581 & 7.5 & 20.8 & - & 7.4 & 84 \\
\hline 19 & 1230 & 3.0 & 600 & 577 & 7.5 & 20.0 & $\cdots$ & 7.4 & 83 \\
\hline 19 & 1229 & 5.1 & 600 & 577 & 7.5 & 19.8 & -- & 7.3 & 82 \\
\hline 19 & 1229 & 9.9 & 600 & 576 & 7.5 & 19.6 & $\cdots$ & 7.3 & 81 \\
\hline 19 & 1228 & 15 & 600 & 574 & 7.5 & 19.6 & $\cdots$ & 7.2 & 80 \\
\hline 19 & 1228 & 20 & 600 & 573 & 7.5 & 19.5 & - & 7.2 & 79 \\
\hline 19 & 1227 & 25 & 600 & 573 & 7.5 & 19.5 & $\cdots$ & 7.1 & 79 \\
\hline 19 & 1227 & 31 & 600 & 573 & 7.5 & 19.5 & $\cdots$ & 7.0 & 77 \\
\hline 19 & 1232 & 0.6 & 900 & 579 & 7.6 & 21.1 & $\cdots$ & 7.7 & 87 \\
\hline 19 & 1232 & 3.3 & 900 & 577 & 7.5 & 20.2 & $\cdots$ & 7.6 & 85 \\
\hline 19 & 1233 & 5.2 & 900 & 577 & 7.5 & 20.1 & 4.5 & 7.5 & 83 \\
\hline 19 & 1233 & 10 & 900 & 574 & 7.5 & 19.7 & - - & 7.3 & 80 \\
\hline 19 & 1234 & 15 & 900 & 572 & 7.5 & 19.6 & $\cdots$ & 7.3 & 80 \\
\hline 19 & 1234 & 20 & 900 & 573 & 7.5 & 19.5 & $\cdots$ & 7.3 & 80 \\
\hline 19 & 1235 & 25 & 900 & 573 & 7.5 & 19.5 & $\cdots$ & 7.2 & 79 \\
\hline 19 & 1235 & 30 & 900 & 573 & 7.5 & 19.5 & - & 7.2 & 80 \\
\hline 19 & 1236 & 33 & 900 & 573 & 7.5 & 19.5 & $\cdots$ & 7.1 & 78 \\
\hline 19 & 1238 & 1.0 & 1,300 & 578 & 7.5 & 20.3 & $\cdots$ & 7.4 & 83 \\
\hline 19 & 1238 & 3.0 & 1,300 & 578 & 7.5 & 20.4 & $\cdots$ & 7.5 & 84 \\
\hline 19 & 1239 & 5.1 & 1,300 & 576 & 7.5 & 20.2 & $-\cdot$ & 7.5 & 83 \\
\hline 19 & 1240 & 10 & 1,300 & 577 & 7.5 & 20.0 & $\cdots$ & 7.3 & 82 \\
\hline 19 & 1240 & 15 & 1,300 & 574 & 7.5 & 19.6 & -- & 7.3 & 81 \\
\hline 19 & 1241 & 20 & 1,300 & 574 & 7.5 & 19.6 & -- & 7.1 & 79 \\
\hline 19 & 1241 & 25 & 1,300 & 574 & 7.5 & 19.6 & - & 7.2 & 80 \\
\hline 19 & 1242 & 30 & 1,300 & 574 & 7.5 & 19.6 & - & 7.2 & 80 \\
\hline 19 & 1242 & 33 & 1,300 & 573 & 7.5 & 19.6 & -- & 7.1 & 78 \\
\hline
\end{tabular}


Table 3. Water-quality data for station 392121081193401, Ohio River at river mile 162.1, June to October, 1995.

[Et $=$ feet $\mu \mu \mathrm{s} / \mathrm{cm}=$ microsiemens per centimeter $;{ }^{\circ} \mathrm{C}=$ degrees Celsius: $\mathrm{mg} / \mathrm{L}=\mathrm{milligrams}$ per liter: - = data not collected]

\begin{tabular}{|c|c|c|c|c|c|c|c|c|c|}
\hline Date & Time & $\begin{array}{l}\text { Sampling } \\
\text { depth } \\
(E t)\end{array}$ & $\begin{array}{c}\text { Sample } \\
\text { location } \\
\text { (ft trom } \\
\text { left bank) }\end{array}$ & $\begin{array}{l}\text { Specific } \\
\text { conduct - } \\
\text { ance } \\
(\mu \mathrm{s} / \mathrm{cm})\end{array}$ & $\begin{array}{l}\mathrm{pH} \\
\text { (stan- } \\
\text { dard } \\
\text { units) }\end{array}$ & $\begin{array}{c}\text { Temper- } \\
\text { ature, } \\
\text { water } \\
\left({ }^{\circ} \mathrm{C}\right)\end{array}$ & $\begin{array}{c}\text { Trans - } \\
\text { parency } \\
\text { (secchi } \\
\text { disk) } \\
\text { (tt) }\end{array}$ & $\begin{array}{l}\text { Dissolved } \\
\text { oxygen } \\
(\mathrm{mg} / \mathrm{L})\end{array}$ & $\begin{array}{c}\text { Dissolved } \\
\text { oxygen } \\
\text { (percent } \\
\text { satura- } \\
\text { tion) }\end{array}$ \\
\hline
\end{tabular}

\begin{tabular}{|c|c|c|c|c|c|c|c|c|c|}
\hline \multicolumn{10}{|l|}{ June } \\
\hline 29 & 1120 & 0.2 & 300 & 352 & 7.3 & 27.4 & $\cdots$ & 7.2 & 93 \\
\hline 29 & 1121 & 3.0 & 300 & 351 & 7.3 & 27.3 & $\cdots$ & 6.7 & 87 \\
\hline 29 & 1121 & 4.8 & 300 & 352 & 7.3 & 27.3 & $\cdots$ & 6.7 & 86 \\
\hline 29 & 1122 & 10 & 300 & 353 & 7.3 & 27.3 & $\cdots$ & 6.7 & 87 \\
\hline 29 & 1123 & 15 & 300 & 349 & 7.2 & 27.3 & $\cdots$ & 6.7 & 87 \\
\hline 29 & 1123 & 20 & 300 & 355 & 7.2 & 27.3 & $\cdots$ & 6.7 & 87 \\
\hline 29 & 1124 & 22 & 300 & 351 & 7.2 & 27.3 & $\cdots$ & 6.7 & 87 \\
\hline 29 & 1115 & 0.5 & 500 & 353 & 7.3 & 27.3 & - & 6.7 & 87 \\
\hline 29 & 1114 & 3.0 & 500 & 352 & 7.3 & 27.3 & 1.5 & 6.7 & 87 \\
\hline 29 & 1114 & 5.1 & 500 & 354 & 7.3 & 27.3 & $\ldots$ & 6.7 & 86 \\
\hline 29 & 1113 & 9.9 & 500 & 354 & 7.2 & 27.3 & $\cdots$ & 6.7 & 86 \\
\hline 29 & 1113 & 16 & 500 & 356 & 7.2 & 27.3 & $\cdots$ & 6.7 & 87 \\
\hline 29 & 1109 & 0.2 & 800 & 352 & 7.3 & 27.4 & $\cdots$ & 7.0 & 90 \\
\hline 29 & 1108 & 3.0 & 800 & 352 & 7.3 & 27.3 & $\cdots$ & 6.8 & 88 \\
\hline 29 & 1108 & 5.0 & 800 & 352 & 7.2 & 27.3 & -- & 6.8 & 88 \\
\hline 29 & 1110 & 10 & 800 & 351 & 7.2 & 27.3 & - & 6.8 & 88 \\
\hline 29 & 1110 & 14 & 800 & 351 & 7.2 & 27.3 & - & 6.7 & 87 \\
\hline 29 & 1109 & 17 & 800 & 354 & 7.2 & 27.3 & $\cdots$ & 6.7 & 87 \\
\hline 29 & 1100 & 0.2 & 1,000 & 356 & 7.1 & 27.0 & $\cdots$ & 7.0 & 89 \\
\hline 29 & 1101 & 3.1 & 1,000 & 352 & 7.3 & 27.4 & $\cdots$ & 6.8 & 88 \\
\hline 29 & 1101 & 4.8 & 1,000 & 354 & 7.3 & 27.4 & $\cdots$ & 6.8 & 88 \\
\hline 29 & 1103 & 8.7 & 1,000 & 355 & 7.3 & 27.4 & - & 6.8 & 87 \\
\hline 29 & 1102 & 14 & 1,000 & 353 & 7.3 & 27.4 & $\cdots$ & 6.8 & 87 \\
\hline \multicolumn{10}{|l|}{ July } \\
\hline 13 & 1232 & 0.9 & 300 & 424 & 7.4 & 27.6 & $\cdots$ & 7.9 & 102 \\
\hline 13 & 1234 & 2.9 & 300 & 424 & 7.4 & 27.6 & $\cdots$ & 8.2 & 106 \\
\hline 13 & 1235 & 4.9 & 300 & 426 & 7.4 & 27.6 & $\cdots$ & 8.0 & 103 \\
\hline 13 & 1235 & 9.9 & 300 & 426 & 7.4 & 27.6 & $\cdots$ & 7.9 & 102 \\
\hline 13 & 1236 & 15 & 300 & 426 & 7.4 & 27.6 & $\cdots$ & 7.9 & 101 \\
\hline 13 & 1237 & 20 & 300 & 425 & 7.4 & 27.6 & . & 7.8 & 101 \\
\hline
\end{tabular}


Table 3. Water-quality data for station 392121081193401, Ohio River at river mile 162.1, June to October, 1995, Continued.

$\left[\mathrm{Et}=\right.$ Eeet $; \mu \mathrm{s} / \mathrm{cm}=$ microsiemens per centimeter: ${ }^{\circ} \mathrm{C}=$ degrees celsius; $\mathrm{mg} / \mathrm{L}=$ milligrams per liter; $\cdots$ = data not collected]

\begin{tabular}{|c|c|c|c|c|c|c|c|c|c|}
\hline Date & Time & $\begin{array}{l}\text { Sampling } \\
\text { depth } \\
\text { (ft) }\end{array}$ & $\begin{array}{c}\text { Sample } \\
\text { location } \\
\text { (Et trom } \\
\text { left bank) }\end{array}$ & $\begin{array}{l}\text { Specific } \\
\text { conduct- } \\
\text { ance } \\
(\mu \mathrm{S} / \mathrm{cm})\end{array}$ & $\begin{array}{l}\mathrm{pH} \\
\text { (stan- } \\
\text { dard } \\
\text { units) }\end{array}$ & $\begin{array}{c}\text { Temper- } \\
\text { ature, } \\
\text { water } \\
\left({ }^{\circ} \mathrm{C}\right)\end{array}$ & $\begin{array}{c}\text { Trans- } \\
\text { parency } \\
\text { (SecchI } \\
\text { disk) } \\
(I t)\end{array}$ & $\begin{array}{l}\text { Dissolved } \\
\text { oxygen } \\
\text { (mg/L) }\end{array}$ & $\begin{array}{c}\text { Dissolv } \\
\text { oxyge } \\
\text { (perce } \\
\text { satur } \\
\text { tion }\end{array}$ \\
\hline \multicolumn{10}{|l|}{ July } \\
\hline 13 & 1155 & 0.6 & 500 & 424 & 7.4 & 27.6 & $\cdots$ & 7.9 & 102 \\
\hline 13 & 1157 & 3.2 & 500 & 425 & 7.4 & 27.6 & 3.0 & 7.9 & 102 \\
\hline 13 & 1157 & 5.4 & 500 & 425 & 7.4 & 27.6 & - & 7.9 & 101 \\
\hline 13 & 1159 & 10 & 500 & 422 & 7.4 & 27.6 & $\cdots$ & 7.8 & 101 \\
\hline 13 & 1217 & 15 & 500 & 423 & 7.4 & 27.6 & - & 8.1 & 104 \\
\hline 13 & 1149 & 0.5 & 800 & 424 & 7.4 & 27.5 & $\cdots$ & 7.7 & 99 \\
\hline 13 & 1149 & 3.0 & 800 & 426 & 7.4 & 27.6 & $\cdots$ & 7.7 & 99 \\
\hline 13 & 1150 & 4.9 & 800 & 426 & 7.4 & 27.6 & $\cdots$ & 7.7 & 99 \\
\hline 13 & 1150 & 10 & 800 & 425 & 7.4 & 27.5 & $\cdots$ & 7.7 & 99 \\
\hline 13 & 1151 & 15 & 800 & 427 & 7.4 & 27.5 & $\cdots$ & 7.7 & 99 \\
\hline 13 & 1141 & 0.4 & 1,000 & 424 & 7.4 & 27.6 & $\cdots$ & 7.7 & 99 \\
\hline 13 & 1142 & 3.0 & 1,000 & 425 & 7.4 & 27.6 & $\cdots$ & 7.8 & 100 \\
\hline 13 & 1142 & 5.1 & 1,000 & 425 & 7.4 & 27.6 & $\cdots$ & 7.8 & 100 \\
\hline 13 & 1143 & 10 & 1,000 & 425 & 7.4 & 27.6 & $\cdots$ & 7.8 & 101 \\
\hline 13 & 1144 & 16 & 1,000 & 425 & 7.4 & 27.6 & $\cdots$ & 7.8 & 100 \\
\hline 13 & 1145 & 18 & 1,000 & 425 & 7.4 & 27.6 & $\cdots$ & 7.7 & 100 \\
\hline 27 & 1020 & 0.5 & 300 & 477 & 7.3 & 28.7 & $\cdots$ & 6.4 & 84 \\
\hline 27 & 1021 & 3.3 & 300 & 475 & 7.3 & 28.7 & $\cdots$ & 6.4 & 84 \\
\hline 27 & 1021 & 4.8 & 300 & 478 & 7.3 & 28.7 & $\cdots$ & 6.5 & 85 \\
\hline 27 & 1022 & 10 & 300 & 483 & 7.3 & 28.7 & $\cdots$ & 6.5 & 85 \\
\hline 27 & 1022 & 15 & 300 & 473 & 7.3 & 28.7 & $\cdots$ & 6.5 & 85 \\
\hline 27 & 1023 & 20 & 300 & 486 & 7.3 & 28.7 & $\cdots$ & 6.5 & 85 \\
\hline 27 & 1023 & 21 & 300 & 479 & 7.3 & 28.7 & $\cdots$ & 6.4 & 85 \\
\hline 27 & 1026 & 0.5 & 500 & 477 & 7.3 & 28.8 & $\cdots$ & 6.5 & 85 \\
\hline 27 & 1026 & 3.1 & 500 & 478 & 7.3 & 28.8 & $\cdots$ & 6.5 & 85 \\
\hline 27 & 1027 & 5.0 & 500 & 476 & 7.3 & 28.7 & $\cdots$ & 6.5 & 85 \\
\hline 27 & 1027 & 10 & 500 & 479 & 7.3 & 28.7 & $\cdots$ & 6.4 & 85 \\
\hline 27 & 1028 & 14 & 500 & 474 & 7.3 & 28.7 & $\cdots$ & 6.4 & 84 \\
\hline
\end{tabular}


Table 3. Water-quality data for station 392121081193401, Ohio River at river mile 162.1, June to October, 1995, Continued.

[ft $=$ feet $; \mu \mathrm{S} / \mathrm{cm}=$ microsiemens per centimeter $;{ }^{\circ} \mathrm{C}=$ degrees celsius;

$\mathrm{mg} / \mathrm{L}=\mathrm{mill}$ igrams per liter: . = data not collected]

\begin{tabular}{|c|c|c|c|c|c|c|c|c|c|}
\hline Date & Time & $\begin{array}{l}\text { Sampling } \\
\text { depth } \\
\text { (ft) }\end{array}$ & $\begin{array}{c}\text { Sample } \\
\text { location } \\
\text { (ft from } \\
\text { lett bank) }\end{array}$ & $\begin{array}{l}\text { Specific } \\
\text { conduct - } \\
\text { ance } \\
(\mu \mathrm{s} / \mathrm{cm})\end{array}$ & $\begin{array}{l}\mathrm{pH} \\
\text { (stan- } \\
\text { dard } \\
\text { units) }\end{array}$ & $\begin{array}{c}\text { Temper- } \\
\text { ature. } \\
\text { water } \\
\left({ }^{\circ} \mathrm{C}\right)\end{array}$ & $\begin{array}{l}\text { Trans - } \\
\text { parency } \\
\text { (Secchi } \\
\text { disk) } \\
\text { (tt) }\end{array}$ & $\begin{array}{c}\text { Dissolved } \\
\text { oxygen } \\
(\mathrm{mg} / \mathrm{L})\end{array}$ & $\begin{array}{c}\text { Dissolved } \\
\text { oxygen } \\
\text { (percent } \\
\text { satura- } \\
\text { tion) }\end{array}$ \\
\hline
\end{tabular}

\begin{tabular}{|c|c|c|c|c|c|c|c|c|c|}
\hline \multicolumn{10}{|l|}{ July } \\
\hline $\begin{array}{r}\text { Ju1y } \\
27\end{array}$ & 1030 & 0.4 & 800 & 476 & 7.3 & 28.7 & $\cdots$ & 6.5 & 85 \\
\hline 27 & 1031 & 3.2 & 800 & 476 & 7.3 & 28.7 & - & 6.5 & 85 \\
\hline 27 & 1031 & 5.0 & 800 & 476 & 7.3 & 28.7 & $\cdots$ & 6.5 & 85 \\
\hline 27 & 1032 & 10 & 800 & 476 & 7.3 & 28.7 & - & 6.5 & 85 \\
\hline 27 & 1032 & 15 & 800 & 477 & 7.3 & 28.7 & - - & 6.4 & 85 \\
\hline 27 & 1033 & 20 & 800 & 475 & 7.3 & 28.7 & $\cdots$ & 6.5 & 85 \\
\hline 27 & 1038 & 0.5 & 1,000 & 479 & 7.4 & 28.8 & $\cdots$ & 6.6 & 86 \\
\hline 27 & 1038 & 3.1 & 1,000 & 478 & 7.3 & 28.7 & $-\cdot$ & 6.6 & 86 \\
\hline 27 & 1039 & 5.0 & 1,000 & 478 & 7.3 & 28.7 & $\cdots$ & 6.6 & 86 \\
\hline 27 & 1039 & 10 & 1,000 & 477 & 7.4 & 28.8 & -- & 6.6 & 86 \\
\hline 27 & 1040 & 13 & 1,000 & 478 & 7.4 & 28.8 & $\cdots$ & 6.5 & 86 \\
\hline \multicolumn{10}{|c|}{ August } \\
\hline 10 & 1315 & 1.1 & 300 & 472 & 7.5 & 29.4 & - & 6.5 & 87 \\
\hline 10 & 1315 & 3.6 & 300 & 479 & 7.5 & 29.3 & $\cdots$ & 6.4 & 85 \\
\hline 10 & 1316 & 5.7 & 300 & 478 & 7.5 & 29.3 & $\cdots$ & 6.4 & 85 \\
\hline 10 & 1316 & 10 & 300 & 482 & 7.5 & 29.3 & - & 6.3 & 84 \\
\hline 10 & 1317 & 15 & 300 & 483 & 7.5 & 29.3 & $\cdots$ & 6.3 & 84 \\
\hline 10 & 1317 & 20 & 300 & 483 & 7.5 & 29.3 & $\cdots$ & 6.3 & 84 \\
\hline 10 & 1318 & 23 & 300 & 477 & 7.5 & 29.3 & $\cdots$ & 6.3 & 83 \\
\hline 10 & 1310 & 1.5 & 500 & 478 & 7.5 & 29.5 & - & 6.4 & 86 \\
\hline 10 & 1310 & 3.5 & 500 & 478 & 7.5 & 29.4 & 2.5 & 6.4 & \\
\hline 10 & 1311 & 5.4 & 500 & 478 & 7.5 & 29.4 & - & 6.3 & 8 \\
\hline 10 & 1311 & 10 & 500 & 482 & 7.5 & 29.3 & $\cdots$ & 6.3 & 8 \\
\hline 10 & 1312 & 15 & 500 & 480 & 7.5 & 29.3 & $\cdots$ & 6.3 & 8 \\
\hline 10 & 1312 & 16 & 500 & 478 & 7.5 & 29.2 & $\cdots$ & 6.2 & 83 \\
\hline 10 & 1305 & 1.1 & 800 & 478 & 7.5 & 29.3 & $\cdots$ & 6.4 & 85 \\
\hline 10 & 1305 & 3.4 & 800 & 478 & 7.5 & 29.3 & $\cdots$ & 6.3 & \\
\hline 10 & 1306 & 5.4 & 800 & 479 & 7.5 & 29.3 & $\cdots$ & 6.3 & 8 \\
\hline 10 & 1306 & 10 & 800 & 479 & 7.5 & 29.3 & $\cdots$ & 6.3 & 8 \\
\hline 10 & 1307 & 15 & 800 & 478 & 7.5 & 29.3 & $\cdots$ & 6.3 & 8 \\
\hline 10 & 1307 & 20 & 800 & 478 & 7.5 & 29.3 & $\cdots$ & 6.3 & \\
\hline
\end{tabular}


Table 3. Water-quality data for station 392121081193401, Ohio River at river mile 162.1, June to October, 1995, Continued.

$\left[\mathrm{ft}=\right.$ feet $\boldsymbol{\mu \mathrm { S }} / \mathrm{cm}=$ microsiemens per centimeter $;{ }^{\circ} \mathrm{C}=$ degrees Celsius; $\mathrm{mg} / \mathrm{L}=\mathrm{milligrams}$ per liter; $\cdots=$ data not collected]

\begin{tabular}{|c|c|c|c|c|c|c|c|c|c|}
\hline Date & Time & $\begin{array}{c}\text { Sampling } \\
\text { depth } \\
\text { (Et) }\end{array}$ & $\begin{array}{c}\text { Sample } \\
\text { location } \\
\text { (ft trom } \\
\text { lett bank) }\end{array}$ & $\begin{array}{l}\text { Specitic } \\
\text { conduct - } \\
\text { ance } \\
(\mu \mathrm{s} / \mathrm{cm})\end{array}$ & $\begin{array}{l}\mathrm{pH} \\
\text { (stan- } \\
\text { dard } \\
\text { units) }\end{array}$ & $\begin{array}{c}\text { Temper- } \\
\text { ature, } \\
\text { water } \\
\left({ }^{\circ} \mathrm{C}\right)\end{array}$ & $\begin{array}{c}\text { Trans - } \\
\text { parency } \\
\text { (secchl } \\
\text { disk) } \\
\text { (ft) }\end{array}$ & $\begin{array}{l}\text { Dissolved } \\
\text { oxygen } \\
(\mathrm{mg} / \mathrm{L})\end{array}$ & $\begin{array}{c}\text { Dissolved } \\
\text { oxygen } \\
\text { (percent } \\
\text { satura- } \\
\text { tron) }\end{array}$ \\
\hline
\end{tabular}

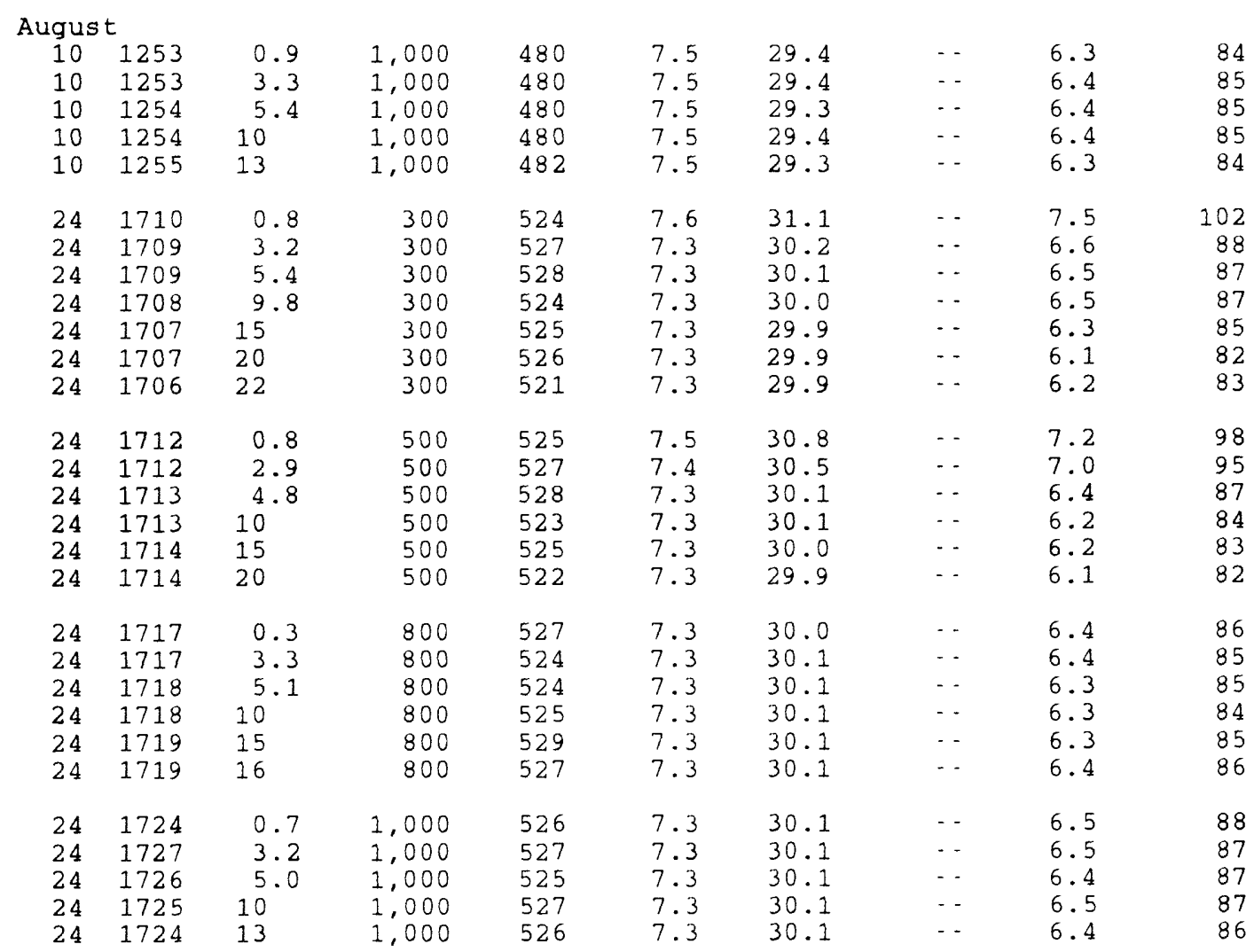


Table 3. Water-quality data for station 392121081193401, Ohio River at river mile 162.1, June to October, 1995, Continued.

\begin{tabular}{|c|c|c|c|c|c|c|c|c|c|}
\hline Date & Time & $\begin{array}{l}\text { Sampling } \\
\text { depth } \\
(t t)\end{array}$ & $\begin{array}{l}\text { Sample } \\
\text { location } \\
\text { (ft from } \\
\text { left bank) }\end{array}$ & $\begin{array}{l}\text { Specific } \\
\text { conduct- } \\
\text { ance } \\
(\mu \mathrm{S} / \mathrm{cm})\end{array}$ & $\begin{array}{l}\text { pH } \\
\text { (stan- } \\
\text { dard } \\
\text { units) }\end{array}$ & $\begin{array}{c}\text { Temper- } \\
\text { ature, } \\
\text { water } \\
\left({ }^{\circ} \mathrm{C}\right)\end{array}$ & $\begin{array}{l}\text { Trans- } \\
\text { parency } \\
\text { (Secchi } \\
\text { disk) } \\
(t t)\end{array}$ & $\begin{array}{l}\text { Dissolved } \\
\text { oxygen } \\
(\mathrm{mg} / \mathrm{L})\end{array}$ & $\begin{array}{c}\text { Dissolv } \\
\text { oxyge } \\
\text { (perce } \\
\text { satur } \\
\text { tion) }\end{array}$ \\
\hline \multicolumn{10}{|c|}{ September } \\
\hline 06 & 1121 & 0.5 & 300 & 576 & 7.2 & 28.1 & $\cdots$ & 7.0 & 90 \\
\hline 06 & 1121 & 3.1 & 300 & 579 & 7.2 & 28.1 & $\ldots$ & 7.0 & 90 \\
\hline 06 & 1122 & 4.9 & 300 & 579 & 7.2 & 28.1 & $\cdots$ & 7.0 & 90 \\
\hline 06 & 1122 & 9.8 & 300 & 580 & 7.2 & 28.1 & - & 6.9 & 89 \\
\hline 06 & 1123 & 15 & 300 & 576 & 7.2 & 28.1 & $\cdots$ & 6.8 & 88 \\
\hline 06 & 1123 & 19 & 300 & 580 & 7.2 & 28.1 & $\cdots$ & 6.7 & 87 \\
\hline 06 & 1116 & 0.2 & 500 & 577 & 7.2 & 28.1 & $\cdots$ & 7.0 & 90 \\
\hline 06 & 1116 & 3.0 & 500 & 577 & 7.2 & 28.1 & -- & 6.9 & 90 \\
\hline 06 & 1117 & 4.8 & 500 & 579 & 7.2 & 28.1 & $\cdots$ & 6.9 & 89 \\
\hline 06 & 1117 & 9.2 & 500 & 580 & 7.2 & 28.1 & $\cdots$ & 6.9 & 90 \\
\hline 06 & 1118 & 15 & 500 & 579 & 7.2 & 28.1 & $\cdots$ & 7.0 & 90 \\
\hline 06 & 1110 & 0.6 & 800 & 577 & 7.2 & 28.1 & -- & 7.0 & 90 \\
\hline 06 & 1110 & 2.9 & 800 & 578 & 7.2 & 28.1 & $\cdots$ & 7.0 & 90 \\
\hline 06 & 1111 & 4.9 & 800 & 577 & 7.2 & 28.1 & - & 6.8 & 88 \\
\hline 06 & 1111 & 9.7 & 800 & 576 & 7.2 & 28.1 & $\cdots$ & 6.8 & 88 \\
\hline 06 & 1112 & 14 & 800 & 575 & 7.2 & 28.1 & - & 6.9 & 89 \\
\hline 06 & 1105 & 0.5 & 1,000 & 578 & 7.2 & 28.1 & - & 6.8 & 88 \\
\hline 06 & 1105 & 3.2 & 1,000 & 577 & 7.2 & 28.1 & - & 6.8 & 88 \\
\hline 06 & 1106 & 4.9 & 1,000 & 577 & 7.2 & 28.1 & -- & 6.7 & 86 \\
\hline 06 & 1106 & 9.8 & 1,000 & 575 & 7.2 & 28.1 & $\cdots$ & 6.8 & 88 \\
\hline 06 & 1107 & 13 & 1,000 & 575 & 7.2 & 28.1 & $\cdots$ & 6.7 & 87 \\
\hline 19 & 1435 & 0.8 & 300 & 651 & 7.3 & 24.3 & $\cdots$ & 6.3 & 76 \\
\hline 19 & 1435 & 3.0 & 300 & 651 & 7.3 & 24.3 & $\cdots$ & 6.5 & 79 \\
\hline 19 & 1436 & 4.8 & 300 & 647 & 7.3 & 24.2 & $\cdots$ & 6.1 & 74 \\
\hline 19 & 1436 & 9.5 & 300 & 650 & 7.3 & 24.2 & $\cdots$ & 6.1 & 74 \\
\hline 19 & 1437 & 15 & 300 & 650 & 7.2 & 24.2 & $\cdots$ & 6.2 & 75 \\
\hline 19 & 1437 & 20 & 300 & 651 & 7.2 & 24.2 & $\cdots$ & 6.1 & 74 \\
\hline 19 & 1438 & 23 & 300 & $64 \overline{6}$ & 7.2 & 24.2 & $\cdots$ & 6.2 & 74 \\
\hline
\end{tabular}


Table 3. Water-quality data for station 392121081193401, ohio River at river mile 162.1, June to october, 1995, Continued.

[ft $=$ feet $; \mu \mathrm{S} / \mathrm{cm}=$ microsiemens per centimeter $;{ }^{\circ} \mathrm{C}=$ degrees Celsius; $\mathrm{mg} / \mathrm{L}=\mathrm{milligrams}$ per liter; $\cdots$ = data not collected]

\begin{tabular}{|c|c|c|c|c|c|c|c|c|c|}
\hline Date & Time & $\begin{array}{l}\text { Sampling } \\
\text { depth } \\
\text { (Et) }\end{array}$ & $\begin{array}{l}\text { Sample } \\
\text { location } \\
\text { (Et from } \\
\text { lett bank) }\end{array}$ & $\begin{array}{l}\text { Specific } \\
\text { conduct- } \\
\text { ance } \\
(\mu \mathrm{s} / \mathrm{cm})\end{array}$ & $\begin{array}{l}\mathrm{pH} \\
\text { (stan- } \\
\text { dard } \\
\text { units) }\end{array}$ & $\begin{array}{c}\text { Temper- } \\
\text { ature, } \\
\text { water } \\
\left({ }^{\circ} \mathrm{C}\right)\end{array}$ & $\begin{array}{c}\text { Trans - } \\
\text { parency } \\
\text { (secchl } \\
\text { disk) } \\
\text { (tt) }\end{array}$ & $\begin{array}{l}\text { Dissolved } \\
\text { oxygen } \\
(\mathrm{mg} / \mathrm{L})\end{array}$ & $\begin{array}{c}\text { Dissolved } \\
\text { oxygen } \\
\text { (percent } \\
\text { satura- } \\
\text { tion) }\end{array}$ \\
\hline
\end{tabular}

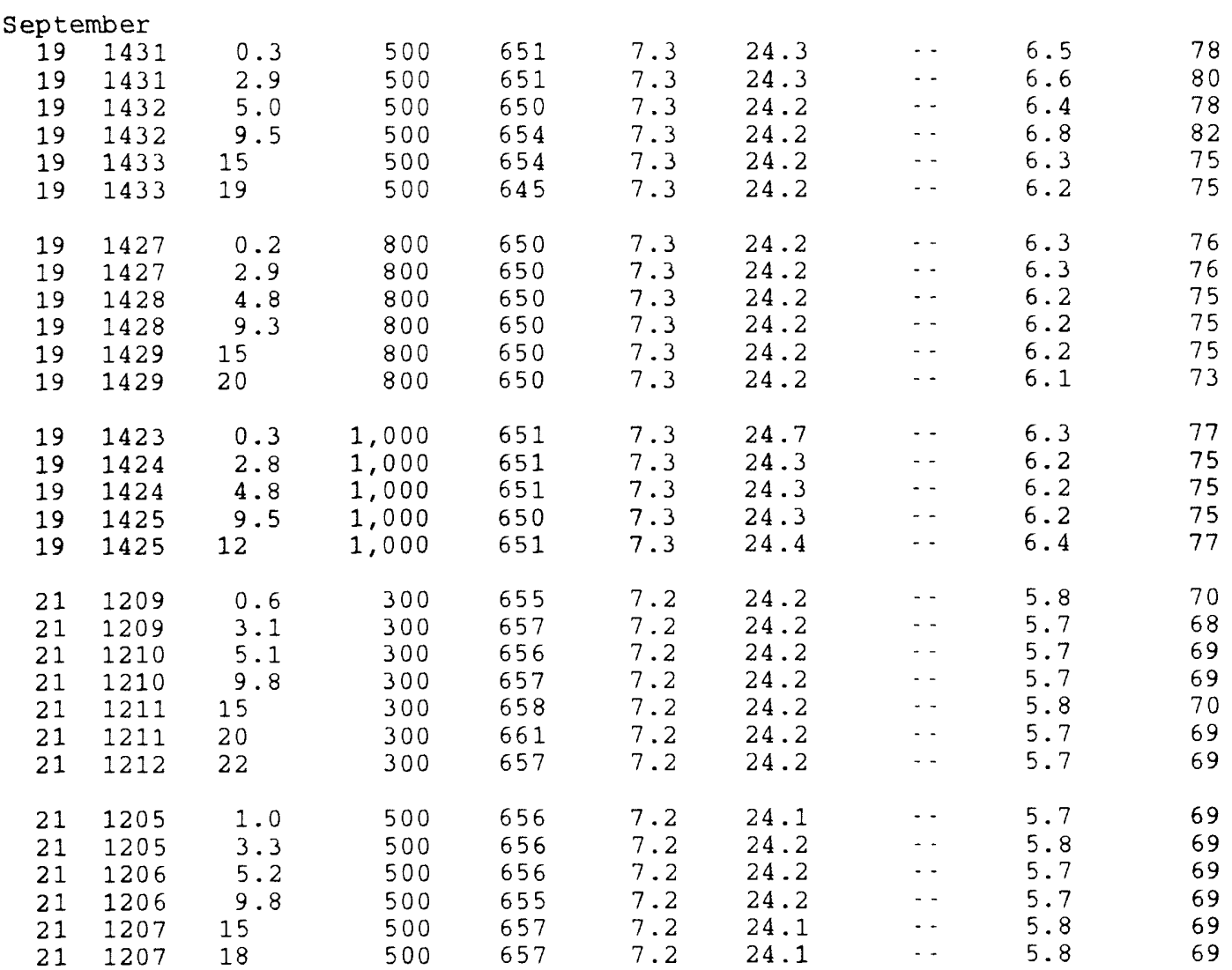


Table 3. Water-quality data for station 392121081193401, Ohio River at river mile 162.1, June to october, 1995, Continued.

$\left[\mathrm{ft}=\mathrm{feet} ; \mu \mathrm{S} / \mathrm{cm}=\right.$ microsiemens per centımeter $;{ }^{\circ} \mathrm{C}=$ degrees Celsius; $\mathrm{mg} / \mathrm{L}=\mathrm{mIlligrams}$ per liter;.. = data not collected]

\begin{tabular}{|c|c|c|c|c|c|c|c|c|c|}
\hline Date & Time & $\begin{array}{l}\text { Sampling } \\
\text { depth } \\
\text { ( } t t)\end{array}$ & $\begin{array}{c}\text { Sample } \\
\text { location } \\
\text { (Et from } \\
\text { left bank) }\end{array}$ & $\begin{array}{l}\text { Speciflc } \\
\text { conduct- } \\
\text { ance } \\
(\mu \mathrm{S} / \mathrm{cm})\end{array}$ & $\begin{array}{l}\text { pH } \\
\text { (stan- } \\
\text { dard } \\
\text { units) }\end{array}$ & $\begin{array}{c}\text { Temper- } \\
\text { ature, } \\
\text { water } \\
\left({ }^{\circ} \mathrm{C}\right)\end{array}$ & $\begin{array}{c}\text { Trans- } \\
\text { parency } \\
\text { (secchi } \\
\text { disk) } \\
\text { (tt) }\end{array}$ & $\begin{array}{c}\text { Dissolved } \\
\text { oxygen } \\
\text { (mg/L) }\end{array}$ & $\begin{array}{c}\text { Dissolved } \\
\text { oxygen } \\
\text { (percent } \\
\text { satura- } \\
\text { tion) }\end{array}$ \\
\hline
\end{tabular}

September

211201

211201

211202

211202

211203

$21 \quad 1203$

$21 \quad 1055$

211056

$21 \quad 1056$

211059

$21 \quad 1059$

$$
\begin{array}{r}
1.0 \\
3.2 \\
5.1 \\
9.9 \\
15 \\
18
\end{array}
$$

0.7

3.1

5.0

9.6

13

october

051740

051740

$05 \quad 1741$

$05 \quad 1741$

$05 \quad 1742$

$05 \quad 1742$

$$
0.9
$$

3.1

5.2

9.9

15

$05 \quad 1744$

051745

$05 \quad 1745$

$05 \quad 1746$

051747

051748

$05 \quad 1747$

18

\section{1}

2.8

5.3

8.3

16

20

25

051750

051750

051751

$05 \quad 1752$

051753

$05 \quad 1752$

1.1

3.7

5.0

9.4

15

18

051759

051759

051800

051800

051801

\section{8}

3.3

5.2

9.9

13

800
800
800
800
800
800
1,000
1,000
1,000
1,000
1,000

657
657
656
656
657
657
656
656
656
654
656

300

300

300

300

300

300

500

500

500

500

500

500

500

800

800

800

800

800

800 $\begin{array}{ll}800 & 564 \\ 800 & 566 \\ 800 & 569 \\ 800 & 573 \\ 800 & 575\end{array}$

$\begin{array}{ll}7.2 & 24.2 \\ 7.2 & 24.2 \\ 7.2 & 24.1 \\ 7.2 & 24.1 \\ 7.2 & 24.1 \\ 7.2 & 24.1 \\ 7.2 & 24.2 \\ 7.2 & 24.2 \\ 7.2 & 24.2 \\ 7.2 & 24.2 \\ 7.2 & 24.2\end{array}$

$7.2 \quad 24.2$

$$
7.5
$$$$
7.4
$$$$
7.4
$$$$
7.4
$$$$
7.4
$$$$
7.4
$$$$
22.1
$$$$
22.1
$$$$
22.1
$$$$
22.1
$$$$
22.1
$$$$
22.1
$$$$
7.4 \quad 22.1
$$$$
7.3 \quad 22.1
$$$$
7.3 \quad 22.1
$$$$
7.4 \quad 22.1
$$$$
7.3 \quad 22.1
$$$$
7.3 \quad 22.1
$$$$
7.3 \quad 22.1
$$$$
7.4 \quad 22.1
$$$$
7.3 \quad 22.1
$$$$
7.3 \quad 22.1
$$$$
7.3 \quad 22.1
$$$$
7.3 \quad 22.1
$$$$
7.3 \quad 22.1
$$$$
7.4 \quad 22.1
$$$$
\begin{aligned}
& 22.1 \\
& 22.1
\end{aligned}
$$$$
\begin{array}{ll}
7.3 & 22.1 \\
7.3 & 22.1
\end{array}
$$$$
7.3 \quad 22.1
$$$$
\begin{array}{ll}
7.3 & 22.1
\end{array}
$$

$\begin{array}{lll}- & 5.8 & 71 \\ - & 5.7 & 69 \\ -. & 5.7 & 69 \\ - & 5.7 & 69 \\ -. & 5.8 & 70 \\ - & 5.8 & 70 \\ - & & \\ -. & 5.8 & 70 \\ - & 5.8 & 70 \\ - & 5.8 & 70 \\ - & 5.8 & 70 \\ & 5.8 & 70\end{array}$

$\begin{array}{lll}\ldots & 6.8 & 79\end{array}$

6.8
$\ldots \quad 6.79$

. 6.796

. 6.7
$\ldots$

- 6.7 .78

$\begin{array}{ll}-6.7 & 78\end{array}$

$\begin{array}{ll}\text {. } & 6.8 \\ \text {. }\end{array}$

. 6.8 .89

. 6.879

$\begin{array}{ll}\ldots & 6.7\end{array}$

. 6.879

- 6.7 .78

- 6.781

$\begin{array}{lll}\cdots & 6.9 & 81\end{array}$

- 6.8 .80

- $6.8 \quad 80$

. 6.8 .89

-6.7
- 68

- 6.781

$\begin{array}{ll}- & 6.7\end{array}$

$\begin{array}{ll}\text {.. } & 6.7 \\ \ldots\end{array}$

. 6.769

$\begin{array}{ll}\text {. } 6.8 & 79\end{array}$

. 6.879 
Table 3. Water-quality data for station 392121081193401, Ohio River at river mile 162.1, June to October, 1995, Continued.

$\left[\mathrm{ft}=\right.$ feet; $\mu \mathrm{S} / \mathrm{cm}=$ microsiemens per centimeter $;{ }^{\circ} \mathrm{C}=$ degrees Celsius; $\mathrm{mg} / \mathrm{L}=$ milifigrams per liter; - = data not collected]

\begin{tabular}{|c|c|c|c|c|c|c|c|c|c|}
\hline Date & Time & $\begin{array}{l}\text { Sampling } \\
\text { depth } \\
\text { (ft) }\end{array}$ & $\begin{array}{l}\text { Sample } \\
\text { location } \\
\text { (Et from } \\
\text { left bank) }\end{array}$ & $\begin{array}{l}\text { Specitic } \\
\text { conduct } \\
\text { ance } \\
(\mu \mathrm{S} / \mathrm{cm})\end{array}$ & $\begin{array}{l}\mathrm{pH} \\
\text { (stan- } \\
\text { dard } \\
\text { units) }\end{array}$ & $\begin{array}{c}\text { Temper- } \\
\text { ature, } \\
\text { water } \\
\left({ }^{\circ} \mathrm{C}\right)\end{array}$ & $\begin{array}{l}\text { Trans- } \\
\text { parency } \\
\text { (Secchi } \\
\text { disk) } \\
\text { (ft) }\end{array}$ & $\begin{array}{l}\text { Dissolved } \\
\text { oxygen } \\
\text { (mg/L) }\end{array}$ & $\begin{array}{c}\text { Dissolved } \\
\text { oxygen } \\
\text { (percent } \\
\text { satura- } \\
\text { tion) }\end{array}$ \\
\hline \multicolumn{10}{|c|}{ October } \\
\hline 19 & 1108 & 0.6 & 300 & 572 & 7.5 & 19.6 & $\cdots$ & 7.5 & 84 \\
\hline 19 & 1108 & 3.3 & 300 & 572 & 7.5 & 19.5 & - - & 7.4 & 82 \\
\hline 19 & 1109 & 5.2 & 300 & 571 & 7.5 & 19.5 & - - & 7.4 & 81 \\
\hline 19 & 1109 & 10 & 300 & 573 & 7.5 & 19.5 & - - & 7.4 & 81 \\
\hline 19 & 1110 & 15 & 300 & 569 & 7.5 & 19.5 & $\cdots$ & 7.4 & 81 \\
\hline 19 & 1110 & 20 & 300 & 570 & 7.5 & 19.5 & $\cdots$ & 7.4 & 82 \\
\hline 19 & 1111 & 22 & 300 & 572 & 7.5 & 19.5 & - & 7.3 & 81 \\
\hline 19 & 1113 & 0.6 & 500 & 569 & 7.5 & 19.7 & $\cdots$ & 7.6 & 84 \\
\hline 19 & 1113 & 3.2 & 500 & 571 & 7.5 & 19.6 & - - & 7.5 & 83 \\
\hline 19 & 1114 & 5.2 & 500 & 571 & 7.5 & 19.6 & 4.0 & 7.5 & 83 \\
\hline 19 & 1114 & 10 & 500 & 573 & 7.5 & 19.5 & - & 7.4 & 82 \\
\hline 19 & 1115 & 15 & 500 & 574 & 7.5 & 19.5 & $\cdots$ & 7.4 & 81 \\
\hline 19 & 1117 & 0.6 & 800 & 569 & 7.5 & 19.7 & $\cdots$ & 7.6 & 85 \\
\hline 19 & 1117 & 3.3 & 800 & 571 & 7.5 & 19.6 & - & 7.5 & 83 \\
\hline 19 & 1118 & 5.4 & 800 & 572 & 7.5 & 19.6 & -- & 7.5 & 82 \\
\hline 19 & 1119 & 10 & 800 & 568 & 7.5 & 19.6 & $\cdots$ & 7.4 & 82 \\
\hline 19 & 1119 & 15 & 800 & 574 & 7.5 & 19.6 & -- & 7.4 & 82 \\
\hline 19 & 1120 & 20 & 800 & 580 & 7.5 & 19.6 & $\cdots$ & 7.4 & 81 \\
\hline 19 & 1120 & 21 & 800 & 571 & 7.5 & 19.6 & -- & 7.3 & 81 \\
\hline 19 & 1122 & 0.9 & 1,000 & 574 & 7.5 & 19.6 & - & 7.5 & 83 \\
\hline 19 & 1122 & 3.2 & 1,000 & 578 & 7.5 & 19.6 & $\cdots$ & 7.5 & 83 \\
\hline 19 & 1123 & 5.1 & 1,000 & 575 & 7.5 & 19.6 & - & 7.5 & 83 \\
\hline 19 & 1123 & 9.9 & 1,000 & 577 & 7.5 & 19.6 & - & 7.5 & 83 \\
\hline 19 & 1124 & 13 & 1,000 & 571 & 7.5 & 19.6 & -- & 7.5 & 83 \\
\hline
\end{tabular}


Table 4. Water-quality data for station 392055081202001, Ohio River at river mile 163.0, June to October, 1995.

$\left[\mathrm{ft}=\mathrm{feet} ; \mu \mathrm{S} / \mathrm{cm}=\mathrm{microsiemens}\right.$ per centimeter ${ }^{\circ} \mathrm{C}=$ degrees Celsius; $\mathrm{mg} / \mathrm{L}=\mathrm{m} l \mathrm{lligrams}$ per liter;. = data not collected]

\begin{tabular}{|c|c|c|c|c|c|c|c|c|c|}
\hline Date & Time & $\begin{array}{l}\text { Sampling } \\
\text { depth } \\
(t t)\end{array}$ & $\begin{array}{c}\text { Sample } \\
\text { location } \\
\text { (Et from } \\
\text { left bank) }\end{array}$ & $\begin{array}{l}\text { Specific } \\
\text { conduct. } \\
\text { ance } \\
(\mu \mathrm{S} / \mathrm{cm})\end{array}$ & $\begin{array}{l}\mathrm{pH} \\
\text { (stan- } \\
\text { dard } \\
\text { units) }\end{array}$ & $\begin{array}{c}\text { Temper- } \\
\text { ature, } \\
\text { water } \\
\left({ }^{\circ} \mathrm{C}\right)\end{array}$ & $\begin{array}{l}\text { Trans - } \\
\text { parency } \\
\text { (Secchi } \\
\text { disk) } \\
(E t)\end{array}$ & $\begin{array}{l}\text { Dissolved } \\
\text { oxygen } \\
(\mathrm{mg} / \mathrm{L})\end{array}$ & $\begin{array}{c}\text { Dissolved } \\
\text { oxygen } \\
\text { (percent } \\
\text { satura- } \\
\text { tion) }\end{array}$ \\
\hline
\end{tabular}

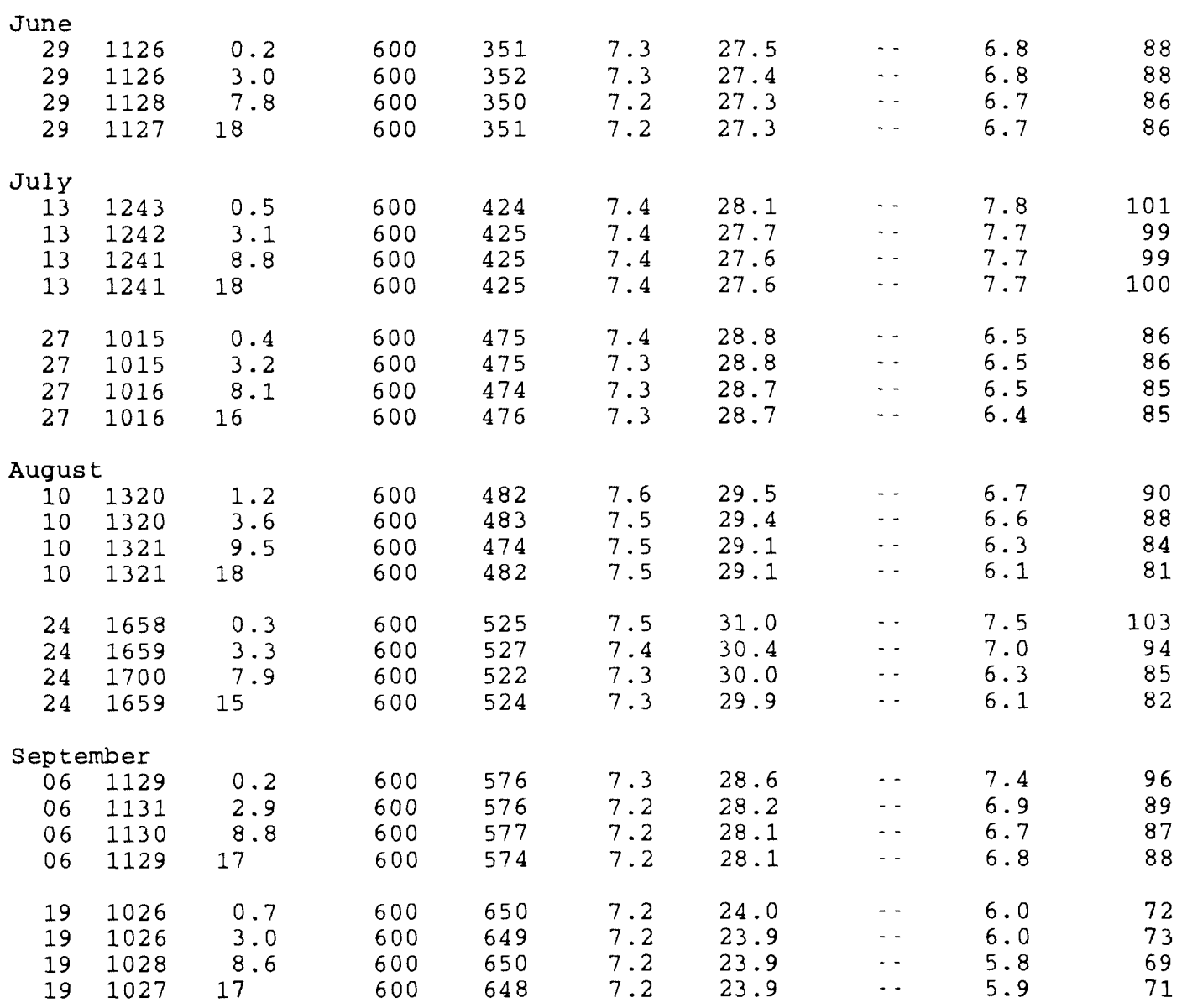


Table 4. Water-quality data for station 392055081202001, Ohio River at river mile 163.0, June to October, 1995, Continued.

[ft $=$ feet $; \mu \mathrm{s} / \mathrm{cm}=$ microsiemens per centimeter $;{ }^{\circ} \mathrm{C}=$ degrees Celsius; $\mathrm{mg} / \mathrm{L}=\mathrm{mili}$ igrams per liter; $\cdot-$ = data not collected]

\begin{tabular}{|c|c|c|c|c|c|c|c|c|c|}
\hline Date & Time & $\begin{array}{l}\text { Sampling } \\
\text { depth } \\
(\mathrm{ft})\end{array}$ & $\begin{array}{c}\text { Sample } \\
\text { location } \\
\text { (tt trom } \\
\text { lett bank) }\end{array}$ & $\begin{array}{l}\text { Specitic } \\
\text { conduct- } \\
\text { ance } \\
(\mu \mathrm{S} / \mathrm{cm})\end{array}$ & $\begin{array}{l}\text { pH } \\
\text { (stan- } \\
\text { dard } \\
\text { units) }\end{array}$ & $\begin{array}{c}\text { Temper- } \\
\text { ature, } \\
\text { water } \\
\left({ }^{\circ} \mathrm{C}\right)\end{array}$ & $\begin{array}{l}\text { Trans- } \\
\text { parency } \\
\text { (SecchI } \\
\text { disk) } \\
\text { (Et) }\end{array}$ & $\begin{array}{c}\text { Dissolved } \\
\text { oxygen } \\
(\mathrm{mg} / \mathrm{L})\end{array}$ & $\begin{array}{c}\text { Dissolved } \\
\text { oxygen } \\
\text { (percent } \\
\text { satura- } \\
\text { tion) }\end{array}$ \\
\hline \multicolumn{10}{|c|}{ September } \\
\hline 20 & 1808 & 0.7 & 600 & 656 & 7.4 & 24.1 & $\cdots$ & 6.1 & 73 \\
\hline 20 & 1808 & 3.2 & 600 & 661 & 7.3 & 24.1 & $\cdots$ & 6.0 & 72 \\
\hline 20 & 1810 & 7.9 & 600 & 663 & 7.3 & 24.1 & $\cdots$ & 5.9 & 71 \\
\hline 20 & 1809 & 16 & 600 & 663 & 7.3 & 24.1 & $\cdots$ & 6.0 & 73 \\
\hline \multicolumn{10}{|c|}{ October } \\
\hline 05 & 1733 & 1.1 & 600 & 565 & 7.4 & 22.1 & $\cdots$ & 6.8 & 79 \\
\hline 05 & 1733 & 3.1 & 600 & 564 & 7.4 & 22.1 & $\cdots$ & 6.8 & 79 \\
\hline 05 & 1735 & 8.7 & 600 & 575 & 7.4 & 22.1 & $\cdots$ & 6.7 & 79 \\
\hline 05 & 1734 & 18 & 600 & 578 & 7.4 & 22.1 & - & 6.7 & 78 \\
\hline 19 & 1102 & 0.8 & 600 & 573 & 7.5 & 19.7 & $\cdots$ & 7.7 & 86 \\
\hline 19 & 1103 & 3.3 & 600 & 572 & 7.5 & 19.6 & $\cdots$ & 7.6 & 84 \\
\hline 19 & 1104 & 8.6 & 600 & 573 & 7.5 & 19.5 & $\cdots$ & 7.3 & 81 \\
\hline 19 & 1103 & 17 & 600 & 574 & 7.5 & 19.5 & $\cdots$ & 7.4 & 82 \\
\hline
\end{tabular}


Table 5. Water-quality data for station 392025081220701, Ohio River at river mile 164.7, June to October, 1995.

[ft $=$ Eeet; $\mu \mathrm{s} / \mathrm{cm}=$ microsiemens per centimeter $;{ }^{\circ} \mathrm{C}=$ degrees Celsius: $\mathrm{mg} / \mathrm{L}=\mathrm{mill}$ igrams per liter; $\ldots$ = data not collected]

\begin{tabular}{|c|c|c|c|c|c|c|c|c|c|}
\hline Date & Time & $\begin{array}{l}\text { Samp I ing } \\
\text { depth } \\
\text { (ft) }\end{array}$ & $\begin{array}{c}\text { Sample } \\
\text { location } \\
\text { (tt trom } \\
\text { left bank) }\end{array}$ & $\begin{array}{l}\text { Specific } \\
\text { conduct. } \\
\text { ance } \\
(\mu \mathrm{s} / \mathrm{cm})\end{array}$ & $\begin{array}{l}\mathrm{pH} \\
\text { (stan- } \\
\text { dard } \\
\text { units) }\end{array}$ & $\begin{array}{l}\text { Temper- } \\
\text { ature, } \\
\text { water } \\
\left({ }^{\circ} \mathrm{C}\right)\end{array}$ & $\begin{array}{c}\text { Trans- } \\
\text { parency } \\
\text { (secchI } \\
\text { dIsk) } \\
(I t)\end{array}$ & $\begin{array}{l}\text { Dissolved } \\
\text { oxygen } \\
\text { (mg/L) }\end{array}$ & $\begin{array}{c}\text { Dissolved } \\
\text { oxygen } \\
\text { (percent } \\
\text { satura- } \\
\text { tion) }\end{array}$ \\
\hline
\end{tabular}

\begin{tabular}{|c|c|c|c|c|c|c|c|c|c|}
\hline \multicolumn{10}{|l|}{ June } \\
\hline 29 & 1133 & 0.4 & 800 & 355 & 7.3 & 27.3 & $-\cdot$ & 6.9 & 88 \\
\hline 29 & 1134 & 3.2 & 800 & 355 & 7.3 & 27.3 & $\cdots$ & 6.9 & 88 \\
\hline 29 & 1135 & 7.0 & 800 & 352 & 7.3 & 27.3 & - & 6.8 & 88 \\
\hline 29 & 1134 & 14 & 800 & 358 & 7.3 & 27.3 & $-\cdot$ & 6.8 & 88 \\
\hline \multicolumn{10}{|l|}{ July } \\
\hline 13 & 1248 & 0.6 & 800 & 424 & 7.4 & 27.8 & $\cdots$ & 7.6 & 98 \\
\hline 13 & 1248 & 3.0 & 800 & 423 & 7.4 & 27.7 & -- & 7.6 & 98 \\
\hline 13 & 1247 & 7.4 & 800 & 425 & 7.4 & 27.5 & - & 7.5 & 96 \\
\hline 13 & 1247 & 15 & 800 & 424 & 7.4 & 27.5 & - & 7.4 & 96 \\
\hline 27 & 1010 & 0.5 & 800 & 477 & 7.4 & 28.7 & $\cdots$ & 6.5 & 86 \\
\hline 27 & 1010 & 3.1 & 800 & 478 & 7.4 & 28.7 & $\cdots$ & 6.6 & 86 \\
\hline 27 & 1011 & 7.1 & 800 & 479 & 7.4 & 28.7 & $\cdots$ & 6.6 & 86 \\
\hline 27 & 1011 & 14 & 800 & 478 & 7.3 & 28.7 & $-\cdot$ & 6.5 & 86 \\
\hline \multicolumn{10}{|c|}{ August } \\
\hline 10 & 1326 & 0.9 & 800 & 449 & 7.6 & 29.3 & $\cdots$ & 7.1 & 94 \\
\hline 10 & 1327 & 3.5 & 800 & 472 & 7.6 & 29.2 & $-\cdot$ & 6.8 & 91 \\
\hline 10 & 1328 & 7.2 & 800 & 473 & 7.5 & 29.0 & $-\cdot$ & 6.5 & 86 \\
\hline 10 & 1327 & 14 & 800 & 474 & 7.5 & 28.9 & $\cdots$ & 6.4 & 84 \\
\hline 24 & 1652 & 0.3 & 800 & 525 & 7.8 & 31.2 & - & 8.7 & 119 \\
\hline 24 & 1652 & 3.2 & 800 & 522 & 7.4 & 30.2 & $\cdots$ & 7.3 & 98 \\
\hline 24 & 1654 & 7.1 & 800 & 522 & 7.3 & 29.9 & $\cdots$ & 6.6 & 88 \\
\hline 24 & 1653 & 14 & 800 & 522 & 7.3 & 29.9 & $\cdots$ & 6.3 & 85 \\
\hline \multicolumn{10}{|c|}{ September } \\
\hline 06 & 1146 & 0.8 & 800 & 573 & 7.2 & 28.5 & $\cdots$ & 7.1 & 93 \\
\hline 06 & 1147 & 3.0 & 800 & 574 & 7.2 & 28.3 & $\cdots$ & 7.0 & 91 \\
\hline 06 & 1148 & 6.3 & 800 & 572 & 7.2 & 28.0 & $\cdots$ & 6.7 & 86 \\
\hline 06 & 1147 & 13 & 800 & 570 & 7.2 & 27.9 & $\cdots$ & 6.6 & 85 \\
\hline 19 & 1021 & 1.1 & 800 & 654 & 7.2 & 23.7 & - & 5.9 & 71 \\
\hline 19 & 1021 & 3.0 & 800 & 653 & 7.2 & 23.7 & $\cdots$ & 5.9 & 71 \\
\hline 19 & 1022 & 6.6 & 800 & 653 & 7.2 & 23.7 & $\cdots$ & 5.8 & 70 \\
\hline 19 & 1022 & 13 & 800 & 651 & 7.2 & 23.7 & $\cdots$ & 5.9 & 70 \\
\hline
\end{tabular}


Table 5. Water-quality data for station 392025081220701, Ohio River at river mile 164.7, June to October, 1995, Continued. $\left[\mathrm{ft}=\mathrm{feet} ; \mu \mathrm{s} / \mathrm{cm}=\right.$ microsiemens per centimeter; ${ }^{\circ} \mathrm{C}=$ degrees celsius;
$\mathrm{mg} / \mathrm{L}=\mathrm{mili}$ igrams per liter; - = data not collected]

\begin{tabular}{|c|c|c|c|c|c|c|c|c|c|}
\hline Date & Time & $\begin{array}{l}\text { Sampling } \\
\text { depth } \\
(\mathrm{ft})\end{array}$ & $\begin{array}{c}\text { Sample } \\
\text { location } \\
\text { (ft trom } \\
\text { left bank) }\end{array}$ & $\begin{array}{l}\text { Specific } \\
\text { conduct- } \\
\text { ance } \\
(\mu \mathrm{S} / \mathrm{cm})\end{array}$ & $\begin{array}{l}\text { pH } \\
\text { (stan- } \\
\text { dard } \\
\text { units) }\end{array}$ & $\begin{array}{c}\text { Temper- } \\
\text { ature, } \\
\text { water } \\
\left({ }^{\circ} \mathrm{C}\right)\end{array}$ & $\begin{array}{l}\text { Trans- } \\
\text { parency } \\
\text { (Secchl } \\
\text { disk) } \\
\text { (士t) }\end{array}$ & $\begin{array}{l}\text { Dissolved } \\
\text { oxygen } \\
(\mathrm{mg} / \mathrm{L})\end{array}$ & $\begin{array}{c}\text { Dissolved } \\
\text { oxygen } \\
\text { (percent } \\
\text { satura- } \\
\text { tion) }\end{array}$ \\
\hline \multicolumn{10}{|c|}{ September } \\
\hline 20 & 1801 & 0.3 & 800 & 660 & 7.4 & 24.0 & $\cdots$ & 6.2 & 74 \\
\hline 20 & 1802 & 3.0 & 800 & 660 & 7.3 & 24.0 & - & 6.2 & 74 \\
\hline 20 & 1803 & 6.8 & 800 & 661 & 7.3 & 24.0 & - - & 6.0 & 73 \\
\hline 20 & 1802 & 14 & 800 & 662 & 7.3 & 24.0 & $\cdots$ & 6.0 & 73 \\
\hline \multicolumn{10}{|c|}{ October } \\
\hline 05 & 1724 & 1.2 & 800 & 558 & 7.4 & 22.1 & $\cdots$ & 6.8 & 79 \\
\hline 05 & 1724 & 3.2 & 800 & 562 & 7.4 & 22.1 & - & 6.8 & 79 \\
\hline 05 & 1726 & 7.2 & 800 & 566 & 7.4 & 22.1 & $\cdots$ & 6.8 & 79 \\
\hline 05 & 1725 & 14 & 800 & 569 & 7.4 & 22.1 & $\cdots$ & 6.8 & 79 \\
\hline 19 & 1058 & 0.6 & 800 & 563 & 7.5 & 19.4 & $\cdots$ & 7.7 & 85 \\
\hline 19 & 1058 & 3.4 & 800 & 567 & 7.5 & 19.3 & $\cdots$ & 7.6 & 84 \\
\hline 19 & 1059 & 6.5 & 800 & 560 & 7.5 & 19.3 & $\cdots$ & 7.4 & 82 \\
\hline 19 & 1059 & 14 & 800 & 569 & 7.5 & 19.3 & $-\cdot$ & 7.4 & 82 \\
\hline
\end{tabular}


Table 6. Water-quality data for station 392110081234201, Ohio River at river mile 166.5, June to October, 1995.

$\left[\mathrm{ft}=\right.$ feet; $\mu \mathrm{S} / \mathrm{cm}=$ microsiemens per centimeter; ${ }^{\circ} \mathrm{C}=$ degrees Celsius; $\mathrm{mg} / \mathrm{L}=\mathrm{milligrams}$ per liter; . = data not collected]

\begin{tabular}{|c|c|c|c|c|c|c|c|c|c|}
\hline Date & Time & $\begin{array}{l}\text { Sampling } \\
\text { depth } \\
\text { (ft) }\end{array}$ & $\begin{array}{l}\text { Sample } \\
\text { location } \\
\text { (Et from } \\
\text { left bank) }\end{array}$ & $\begin{array}{l}\text { Specific } \\
\text { conduct } \\
\text { ance } \\
(\mu \mathrm{S} / \mathrm{cm})\end{array}$ & $\begin{array}{l}\text { pH } \\
\text { (stan- } \\
\text { dard } \\
\text { units) }\end{array}$ & $\begin{array}{c}\text { Temper- } \\
\text { ature, } \\
\text { water } \\
\left({ }^{\circ} \mathrm{C}\right)\end{array}$ & $\begin{array}{l}\text { Trans - } \\
\text { parency } \\
\text { (Secchi } \\
\text { disk) } \\
(\mathrm{tt})\end{array}$ & $\begin{array}{l}\text { Dissolved } \\
\text { oxygen } \\
(\mathrm{mg} / \mathrm{L})\end{array}$ & $\begin{array}{c}\text { Dissolved } \\
\text { oxygen } \\
\text { (percent } \\
\text { satura- } \\
\text { tion) }\end{array}$ \\
\hline
\end{tabular}

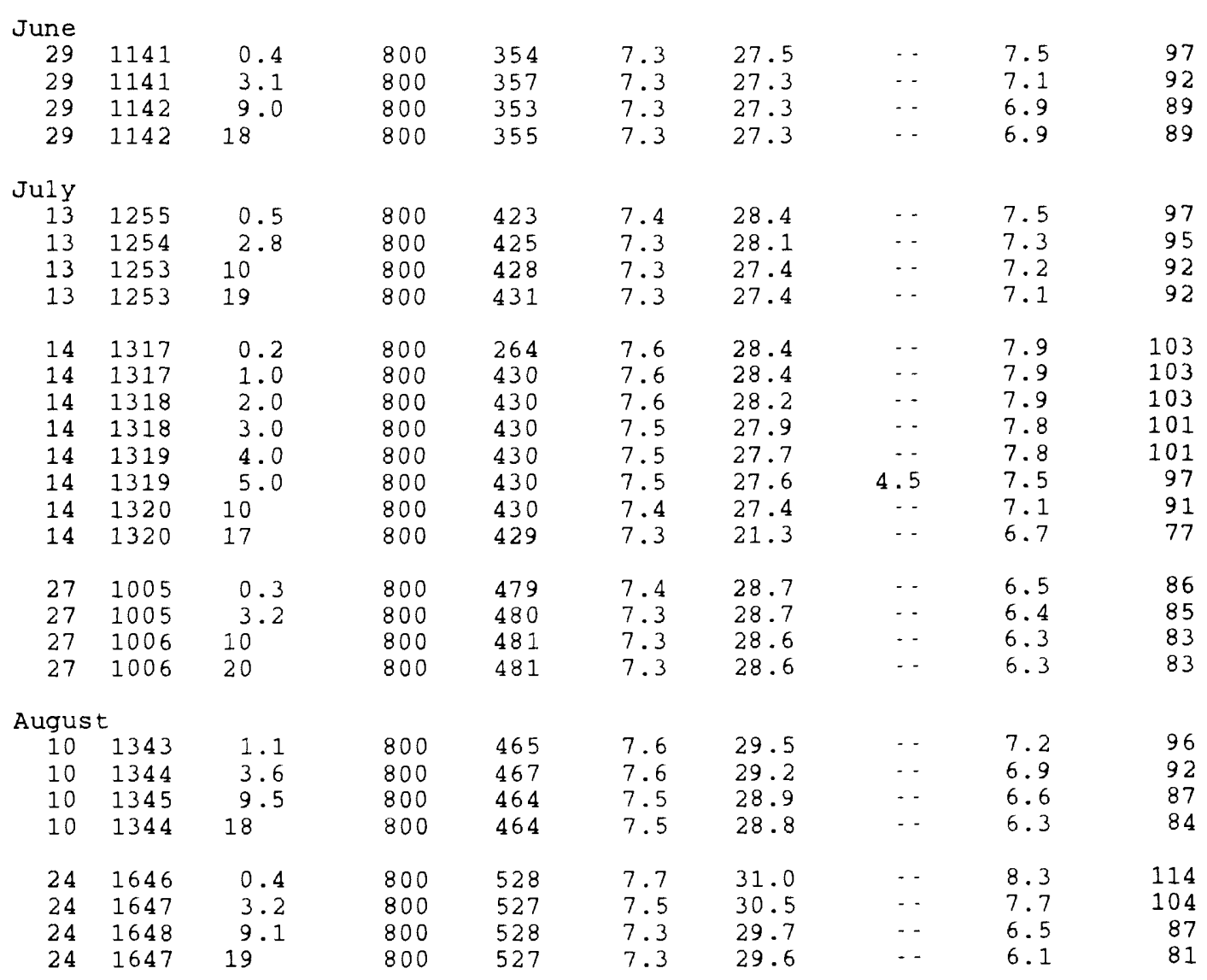


Table 6. Water-quality data for station 392110081234201, Ohio River at river mile 166.5, June to October, 1995, Continued.

[ft $=$ Eeet $; \mu \mathrm{s} / \mathrm{cm}=$ microsiemens per centimeter $;{ }^{\circ} \mathrm{C}=$ degrees Celsius; $\mathrm{mg} / \mathrm{L}=\mathrm{mili}$ igrams per liter; $\cdots$ = data not collected]

\begin{tabular}{|c|c|c|c|c|c|c|c|c|c|}
\hline Date & Time & $\begin{array}{l}\text { Sampling } \\
\text { depth } \\
\text { (Et) }\end{array}$ & $\begin{array}{c}\text { Sample } \\
\text { location } \\
\text { (ft trom } \\
\text { left bank) }\end{array}$ & $\begin{array}{l}\text { Specific } \\
\text { conduct- } \\
\text { ance } \\
(\mu \mathrm{S} / \mathrm{cm})\end{array}$ & $\begin{array}{l}\text { pH } \\
\text { (stan- } \\
\text { dard } \\
\text { units) }\end{array}$ & $\begin{array}{l}\text { Temper- } \\
\text { ature, } \\
\text { water } \\
\left({ }^{\circ} \mathrm{C}\right)\end{array}$ & $\begin{array}{l}\text { Trans - } \\
\text { parency } \\
\text { (Secchl } \\
\text { disk) } \\
\text { (ft) }\end{array}$ & $\begin{array}{c}\text { Dissolved } \\
\text { oxygen } \\
\text { (mg/L) }\end{array}$ & $\begin{array}{c}\text { Dissolved } \\
\text { oxygen } \\
\text { (percent } \\
\text { satura. } \\
\text { tion) }\end{array}$ \\
\hline
\end{tabular}

September

$06 \quad 1152$

061153

$0.5 \quad 800$

\title{
800
800
}

800

800

\section{9}

7.2

28.0

- 7.1

7.0

569
566

7.2

27.9

$-$

6.5

564

$7 \cdot \frac{1}{7}$

$27 \cdot 4$

-

6.6

061153

800

800

654

654

$7.2 \quad 23.7$

- 6.1

$\begin{array}{ll}\cdots & 6.1 \\ \cdots & 6.0\end{array}$

$-$

5.9
5.9

800
800

653

$\begin{array}{ll}7.2 & 23.7\end{array}$

$7.2 \quad 23.7$

$\cdots$

.. 6.2

6.2
6.1

658

7.4

23.9

-

6. $\frac{1}{0}$

661

$7 \cdot 3$

23.9

800

800

658

$7 \cdot 3$

23.9

$\cdots$

6. 1

$-$

6.9

$7.4 \quad 22.1$

$7.4 \quad 22.1$

$7.4 \quad 22.1$

$-$

6.9

.. 6.8

- 6.8

573

$7.4 \quad 22.1$

- 7.6

$7.5 \quad 19.2$

556

$\begin{array}{ll}7.5 & 19.2 \\ 7.5 & 19.1\end{array}$

-

$\cdots$

7.5

$\begin{array}{lll}556 & 7.5 & 19.1\end{array}$

$\cdots$

7.4

7.4

80

$7.5 \quad 19.1$

7.4

79

79

84

83

81

$\begin{array}{ll}800 & 556 \\ 800 & 556\end{array}$

\begin{abstract}
19.1
\end{abstract}

\author{
(n)
}

81 
Table 7. Water-quality data for station 392318081243001, Ohio River at river mile 169.1, main channel, June to October, 1995.

[ft $=$ feet; $\mu \mathrm{S} / \mathrm{cm}=$ microsiemens per centımeter; ${ }^{\circ} \mathrm{C}=$ degrees Celsius: $\mathrm{mg} / \mathrm{L}=\mathrm{milligrams}$ per liter; .. = data not coliected]

\begin{tabular}{|c|c|c|c|c|c|c|c|c|c|}
\hline Date & Tume & $\begin{array}{l}\text { Sampling } \\
\text { depth } \\
\text { (ft) }\end{array}$ & $\begin{array}{l}\text { Sample } \\
\text { location } \\
\text { (Et trom } \\
\text { lett bank) }\end{array}$ & $\begin{array}{l}\text { Specitic } \\
\text { conduct- } \\
\text { ance } \\
(\mu \mathrm{s} / \mathrm{cm})\end{array}$ & $\begin{array}{l}\text { pH } \\
\text { (stan- } \\
\text { dard } \\
\text { units) }\end{array}$ & $\begin{array}{c}\text { Temper- } \\
\text { ature, } \\
\text { water } \\
\left({ }^{\circ} \mathrm{C}\right)\end{array}$ & $\begin{array}{c}\text { Trans- } \\
\text { parency } \\
\text { (secchl } \\
\text { disk) } \\
\text { (tt) }\end{array}$ & $\begin{array}{l}\text { Dissolved } \\
\text { oxygen } \\
(\mathrm{mg} / \mathrm{L})\end{array}$ & $\begin{array}{c}\text { Dissolved } \\
\text { oxygen } \\
\text { (percent } \\
\text { satura- } \\
\text { tion) }\end{array}$ \\
\hline
\end{tabular}

June

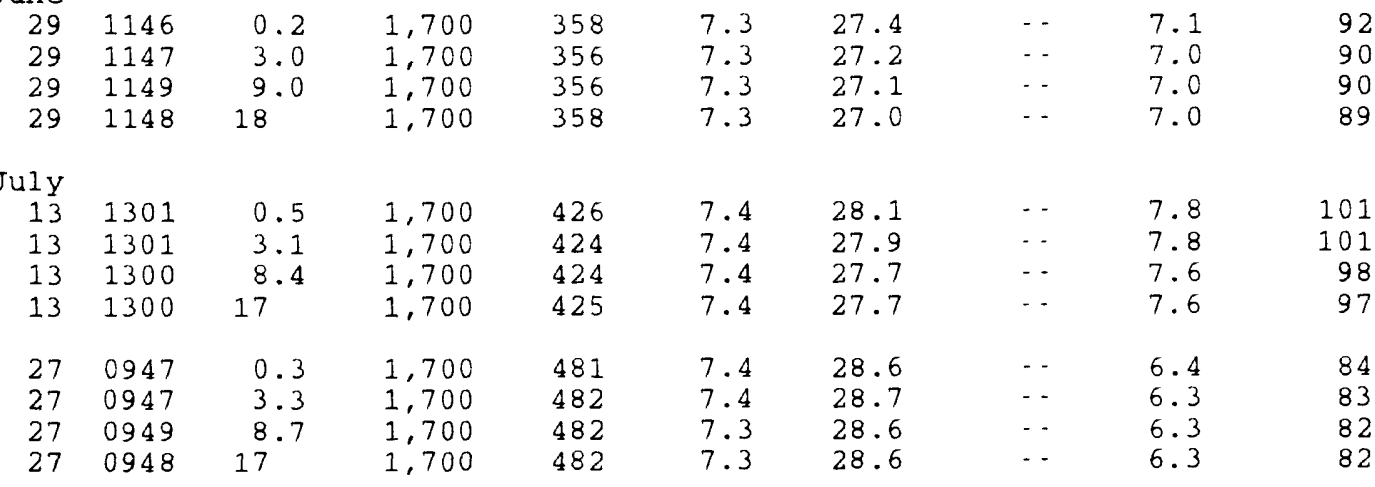

August

\begin{tabular}{|c|c|c|c|c|c|c|c|c|c|}
\hline 10 & 1356 & 1.1 & 1,700 & 433 & 7.6 & 28.5 & $\cdots$ & 7.1 & 93 \\
\hline 10 & 1356 & 3.8 & 1,700 & 442 & 7.6 & 28.8 & $\cdots$ & 7.2 & 95 \\
\hline 10 & 1357 & 9.6 & 1,700 & 435 & 7.5 & 28.4 & $-\cdot$ & 6.9 & 91 \\
\hline 10 & 1357 & 18 & 1,700 & 407 & 7.5 & 27.5 & $\cdots$ & 6.8 & 88 \\
\hline 24 & 1636 & 0.5 & 1,700 & 519 & 7.7 & 31.1 & $\cdots$ & 8.5 & 116 \\
\hline 24 & 1636 & 3.2 & 1,700 & 531 & 7.7 & 31.0 & $\cdots$ & 8.4 & 114 \\
\hline 24 & 1638 & 8.6 & 1,700 & 531 & 7.4 & 30.1 & $-\cdot$ & 7.0 & 95 \\
\hline 24 & 1637 & 17 & 1,700 & 531 & 7.3 & 29.9 & $\cdots$ & 6.5 & 87 \\
\hline epte & nber & & & & & & & & \\
\hline 06 & 1159 & 0.3 & 1,700 & 554 & 7.2 & 28.0 & $\cdots$ & 7.6 & 98 \\
\hline 06 & 1200 & 3.1 & 1,700 & 556 & 7.2 & 27.8 & $\cdots$ & 7.6 & 98 \\
\hline 06 & 1201 & 8.4 & 1,700 & 557 & 7.2 & 27.6 & $\cdots$ & 7.5 & 96 \\
\hline 06 & 1200 & 17 & 1.700 & 555 & 7.2 & 27.6 & $\cdots$ & 7.5 & 96 \\
\hline 19 & 1008 & 0.8 & 1,700 & 661 & 7.2 & 23.7 & $\cdots$ & 6.1 & 73 \\
\hline 19 & 1009 & 3.0 & 1.700 & 665 & 7.2 & 23.7 & $\cdots$ & 5.9 & 71 \\
\hline 19 & 1010 & 8.1 & 1.700 & 665 & 7.2 & 23.6 & $\cdots$ & 5.9 & 71 \\
\hline 19 & 1009 & 16 & 1,700 & 661 & 7.2 & 23.6 & $\cdots$ & 6.0 & 71 \\
\hline
\end{tabular}


Table 7. Water-quality data for station 392318081243001, Ohio River at river mile 169.1, main channel, June to october, 1995, Continued.

$\left[\mathrm{ft}=\mathrm{feet} ; \mu \mathrm{s} / \mathrm{cm}=\right.$ microsiemens per centimeter $;{ }^{\circ} \mathrm{C}=$ degrees Celsius;

$\mathrm{mg} / \mathrm{L}=\mathrm{mlli}$ igrams per liter; $\cdots$ = data not collected]

\begin{tabular}{|c|c|c|c|c|c|c|c|c|c|}
\hline Date & Time & $\begin{array}{l}\text { Sampling } \\
\text { depth } \\
\text { (Et) }\end{array}$ & $\begin{array}{c}\text { Sample } \\
\text { location } \\
\text { (tt Erom } \\
\text { lett bank) }\end{array}$ & $\begin{array}{l}\text { Specific } \\
\text { conduct } \\
\text { ance } \\
(\mu S / \mathrm{cm})\end{array}$ & $\begin{array}{l}\mathrm{pH} \\
\text { (stan- } \\
\text { dard } \\
\text { units) }\end{array}$ & $\begin{array}{c}\text { Temper- } \\
\text { ature. } \\
\text { water } \\
\left({ }^{\circ} \mathrm{C}\right)\end{array}$ & $\begin{array}{l}\text { Trans - } \\
\text { parency } \\
\text { (Secchi } \\
\text { disk) } \\
\text { (ft) }\end{array}$ & $\begin{array}{l}\text { Dissolved } \\
\text { oxygen } \\
(\mathrm{mg} / \mathrm{L})\end{array}$ & $\begin{array}{c}\text { Dissolved } \\
\text { oxygen } \\
\text { (percent } \\
\text { satura- } \\
\text { tion) }\end{array}$ \\
\hline
\end{tabular}

\section{September}

$20 \quad 1746$

$20 \quad 1746$

$20 \quad 1748$

$0.6 \quad 1,700$

661

$3.21,700$

659

$\begin{array}{ll}7.4 & 23.7\end{array}$

- 6.3

6.3
6.3

23.8

$\begin{array}{lll}661 & 7.3 & 23.8\end{array}$

$17 \quad 1,700$

662

7.3

23.8

$-$

$6 \cdot 3$

76

$20 \quad 1747$

october

\begin{tabular}{|c|c|c|c|c|c|c|c|c|}
\hline $\begin{array}{ll}05 & 2003 \\
05 & 2003 \\
05 & 2004 \\
05 & 2004\end{array}$ & $\begin{array}{l}0.6 \\
3.2 \\
7.7 \\
15\end{array}$ & $\begin{array}{l}1,700 \\
1,700 \\
1,700 \\
1,700\end{array}$ & $\begin{array}{l}553 \\
557 \\
562 \\
564\end{array}$ & $\begin{array}{l}7.4 \\
7.3 \\
7.4 \\
7.4\end{array}$ & $\begin{array}{l}21.9 \\
22.0 \\
22.0 \\
22.0\end{array}$ & $\begin{array}{l}\cdots \\
\cdots \\
\cdots\end{array}$ & $\begin{array}{l}6.8 \\
6.8 \\
6.9 \\
6.9\end{array}$ & $\begin{array}{l}79 \\
79 \\
80 \\
80\end{array}$ \\
\hline $\begin{array}{ll}19 & 1047 \\
19 & 1047 \\
19 & 1048 \\
19 & 1048\end{array}$ & $\begin{array}{r}0.6 \\
2.9 \\
8.7 \\
16\end{array}$ & $\begin{array}{l}1,700 \\
1,700 \\
1,700 \\
1,700\end{array}$ & $\begin{array}{l}552 \\
552 \\
552 \\
552\end{array}$ & $\begin{array}{l}7.5 \\
7.5 \\
7.5 \\
7.5\end{array}$ & $\begin{array}{l}19.2 \\
19.2 \\
19.2 \\
19.2\end{array}$ & $\begin{array}{l}- \\
\cdots \\
- \\
-\end{array}$ & $\begin{array}{l}7.8 \\
7.6 \\
7.4 \\
7.5\end{array}$ & $\begin{array}{l}85 \\
83 \\
82 \\
82\end{array}$ \\
\hline
\end{tabular}


Table 8. Water-quality data for station 392313081244601, Ohio River at river mile 169.1, back channel, June to October, 1995.

[ft $=$ feet; $\mu \mathrm{s} / \mathrm{cm}=$ microsiemens per centımeter; ${ }^{\circ} \mathrm{C}=$ degrees Celsius; $\mathrm{mg} / \mathrm{L}=\mathrm{m} I l i$ igrams per liter; $\cdots$ = data not collected]

\begin{tabular}{|c|c|c|c|c|c|c|c|c|c|}
\hline Date & Time & $\begin{array}{l}\text { Sampling } \\
\text { depth } \\
\text { (ft) }\end{array}$ & $\begin{array}{c}\text { Sample } \\
\text { location } \\
\text { (tt trom } \\
\text { left bank) }\end{array}$ & $\begin{array}{l}\text { Specific } \\
\text { conduct- } \\
\text { ance } \\
(\mu \mathrm{s} / \mathrm{cm})\end{array}$ & $\begin{array}{l}\mathrm{pH} \\
\text { (stan- } \\
\text { dard } \\
\text { units) }\end{array}$ & $\begin{array}{c}\text { Temper- } \\
\text { ature, } \\
\text { water } \\
\left({ }^{\circ} \mathrm{C}\right)\end{array}$ & $\begin{array}{l}\text { Trans- } \\
\text { parency } \\
\text { (Secchl } \\
\text { disk) } \\
\text { (tt) }\end{array}$ & $\begin{array}{l}\text { Dissolved } \\
\text { oxygen } \\
(\mathrm{mg} / \mathrm{L})\end{array}$ & $\begin{array}{c}\text { D)ssolved } \\
\text { oxygen } \\
\text { (percent } \\
\text { satura- } \\
\text { tion) }\end{array}$ \\
\hline \multicolumn{10}{|l|}{ June } \\
\hline 29 & 1157 & 0.4 & 400 & 360 & 7.3 & 27.3 & $\cdots$ & 6.9 & 88 \\
\hline 29 & 1156 & 3.1 & 400 & 359 & 7.3 & 27.3 & - & 6.8 & 88 \\
\hline 29 & 1156 & 5.0 & 400 & 359 & 7.3 & 27.2 & $\cdots$ & 6.8 & 88 \\
\hline 29 & 1157 & 11 & 400 & 356 & 7.2 & 27.2 & - & 6.8 & 87 \\
\hline \multicolumn{10}{|l|}{ Ju1y } \\
\hline 13 & 1306 & 0.5 & 400 & 425 & 7.4 & 28.5 & $\cdots$ & 7.9 & 104 \\
\hline 13 & 1305 & 3.1 & 400 & 425 & 7.4 & 28.3 & $\cdots$ & 7.8 & 102 \\
\hline 13 & 1305 & 10 & 400 & 427 & 7.3 & 27.6 & $\cdots$ & 7.4 & 95 \\
\hline 13 & 1304 & 20 & 400 & 424 & 7.3 & 27.6 & $\cdots$ & 7.3 & 95 \\
\hline 27 & 0951 & 0.4 & 400 & 482 & 7.3 & 28.6 & $\cdots$ & 6.3 & 83 \\
\hline 27 & 0951 & 3.2 & 400 & 482 & 7.3 & 28.6 & - & 6.2 & 82 \\
\hline 27 & 0952 & 5.8 & 400 & 482 & 7.3 & 28.5 & $\cdots$ & 6.2 & 81 \\
\hline 27 & 0952 & 12 & 400 & 482 & 7.3 & 28.5 & $\cdots$ & 6.2 & 81 \\
\hline \multicolumn{10}{|c|}{ August } \\
\hline 10 & 1350 & 1.1 & 400 & 453 & 7.6 & 29.6 & $\cdots$ & 7.2 & 96 \\
\hline 10 & 1350 & 3.6 & 400 & 454 & 7.6 & 28.9 & $\cdots$ & 7.2 & 95 \\
\hline 10 & 1353 & 9.5 & 400 & 420 & 7.4 & 27.6 & $\cdots$ & 6.4 & 83 \\
\hline 10 & 1353 & 19 & 400 & 252 & 7.3 & 23.6 & -- & 6.2 & 75 \\
\hline 24 & 1640 & 0.2 & 400 & 531 & 7.6 & 31.2 & $\cdots$ & 8.0 & 110 \\
\hline 24 & 1640 & 3.2 & 400 & 531 & 7.4 & 30.5 & $\cdots$ & 7.2 & 97 \\
\hline 24 & 1641 & 9.0 & 400 & 531 & 7.3 & 29.9 & $\cdots$ & 6.5 & 87 \\
\hline 24 & 1641 & 19 & 400 & 530 & 7.2 & 29.8 & $-\cdot$ & 6.1 & 81 \\
\hline \multicolumn{10}{|c|}{ September } \\
\hline 06 & 1203 & 0.2 & 400 & 551 & 7.2 & 28.0 & $\cdots$ & 7.5 & 97 \\
\hline 06 & 1204 & 2.9 & 400 & 552 & 7.2 & 27.6 & $\cdots$ & 7.3 & 93 \\
\hline 06 & 1206 & 5.4 & 400 & 554 & 7.2 & 27.5 & $\cdots$ & 7.2 & 92 \\
\hline 06 & 1205 & 11 & 400 & 554 & 7.1 & 27.4 & $\cdots$ & 6.9 & 88 \\
\hline 19 & 1004 & 0.3 & 400 & 664 & 7.2 & 23.6 & $\cdots$ & 6.2 & 74 \\
\hline 19 & 1005 & 3.0 & 400 & 662 & 7.2 & 23.6 & -- & 6.0 & 72 \\
\hline 19 & 1006 & 5.4 & 400 & 665 & 7.2 & 23.6 & $\cdots$ & 5.9 & 71 \\
\hline 19 & 1005 & 11 & 400 & 663 & 7.2 & 23.6 & $\cdots$ & 5.9 & 70 \\
\hline
\end{tabular}


Table 8. Water-quality data for station 392313081244601, Ohio River at river mile 169.1, back channel, June to October, 1995, Continued.

[ft $=$ feet $; \mu \mathrm{s} / \mathrm{cm}=$ microsiemens per centimeter $;{ }^{\circ} \mathrm{C}=$ degrees Celsius: $\mathrm{mg} / \mathrm{L}=\mathrm{mili}$ igrams per liter; $\cdot-$ = data not collected]

\begin{tabular}{|c|c|c|c|c|c|c|c|c|c|}
\hline Date & Time & $\begin{array}{l}\text { Sampling } \\
\text { depth } \\
\text { (ft) }\end{array}$ & $\begin{array}{l}\text { Sample } \\
\text { location } \\
\text { (tt trom } \\
\text { left bank) }\end{array}$ & $\begin{array}{l}\text { Specific } \\
\text { conduct. } \\
\text { ance } \\
(\mu \mathrm{S} / \mathrm{cm})\end{array}$ & $\begin{array}{l}\text { pH } \\
\text { (stan- } \\
\text { dard } \\
\text { units) }\end{array}$ & $\begin{array}{c}\text { Temper- } \\
\text { ature, } \\
\text { water } \\
\left({ }^{\circ} \mathrm{C}\right)\end{array}$ & $\begin{array}{c}\text { Trans. } \\
\text { parency } \\
\text { (Secchl } \\
\text { disk) } \\
(\mathrm{t} t)\end{array}$ & $\begin{array}{l}\text { Dissolved } \\
\text { oxygen } \\
\text { (mg/L) }\end{array}$ & $\begin{array}{c}\text { Dissolved } \\
\text { oxygen } \\
\text { (percent } \\
\text { satura- } \\
\text { tion) }\end{array}$ \\
\hline \multicolumn{10}{|c|}{ september } \\
\hline 20 & 1823 & 0.6 & 400 & 659 & 7.3 & 23.8 & - & 6.2 & 74 \\
\hline 20 & 1823 & 3.0 & 400 & 660 & 7.3 & 23.8 & $\cdots$ & 6.1 & 74 \\
\hline 20 & 1825 & 6.4 & 400 & 662 & 7.3 & 23.8 & - & 6.0 & 72 \\
\hline 20 & 1824 & 12 & 400 & 659 & 7.3 & 23.8 & $\cdots$ & 6.1 & 73 \\
\hline \multicolumn{10}{|c|}{ October } \\
\hline 05 & 1657 & 0.7 & 400 & 549 & 7.4 & 21.9 & $\cdots$ & 6.8 & 79 \\
\hline 05 & 1657 & 2.5 & 400 & 558 & 7.3 & 21.9 & $\cdots$ & 6.8 & 79 \\
\hline 05 & 1659 & 6.6 & 400 & 559 & 7.3 & 21.9 & $\cdots$ & 6.7 & 77 \\
\hline 05 & 1658 & 12 & 400 & 559 & 7.3 & 21.9 & $\cdots$ & 6.6 & 77 \\
\hline 19 & 1044 & 0.8 & 400 & 551 & 7.5 & 19.3 & $\cdots$ & 7.7 & 85 \\
\hline 19 & 1044 & 3.5 & 400 & 551 & 7.4 & 19.2 & -- & 7.5 & 83 \\
\hline 19 & 1045 & 5.6 & 400 & 551 & 7.4 & 19.2 & $\cdots$ & 7.3 & 80 \\
\hline 19 & 1045 & 11 & 400 & 551 & 7.4 & 19.2 & - & 7.4 & 81 \\
\hline
\end{tabular}


Table 9. Water-quality data for station 392419081255001, Ohio River at river mile 170.8, main channel, June to october, 1995.

IEt $=$ Eeet; $\mu \mathrm{S} / \mathrm{cm}=$ microsiemens per centımeter $;{ }^{\circ} \mathrm{C}=$ degrees Celsius; $\mathrm{mg} / \mathrm{L}=\mathrm{milligrams}$ per liter; $\cdots$ = data not coliected]

\begin{tabular}{|c|c|c|c|c|c|c|c|c|c|}
\hline Date & Time & $\begin{array}{l}\text { Sampling } \\
\text { depth } \\
\text { (ft) }\end{array}$ & $\begin{array}{l}\text { Sample } \\
\text { location } \\
\text { (Et from } \\
\text { left bank) }\end{array}$ & $\begin{array}{l}\text { specific } \\
\text { conduct- } \\
\text { ance } \\
(\mu \mathrm{S} / \mathrm{cm})\end{array}$ & $\begin{array}{l}\mathrm{pH} \\
\text { (stan- } \\
\text { dard } \\
\text { units) }\end{array}$ & $\begin{array}{c}\text { Temper- } \\
\text { ature, } \\
\text { water } \\
\left({ }^{\circ} \mathrm{C}\right)\end{array}$ & $\begin{array}{l}\text { Trans - } \\
\text { parency } \\
\text { (Secchl } \\
\text { disk) } \\
\text { (Et) }\end{array}$ & $\begin{array}{l}\text { Dissolved } \\
\text { oxygen } \\
\text { (mg/L) }\end{array}$ & $\begin{array}{c}\text { Dissolved } \\
\text { oxygen } \\
\text { (percent } \\
\text { satura- } \\
\text { tion) }\end{array}$ \\
\hline
\end{tabular}

June

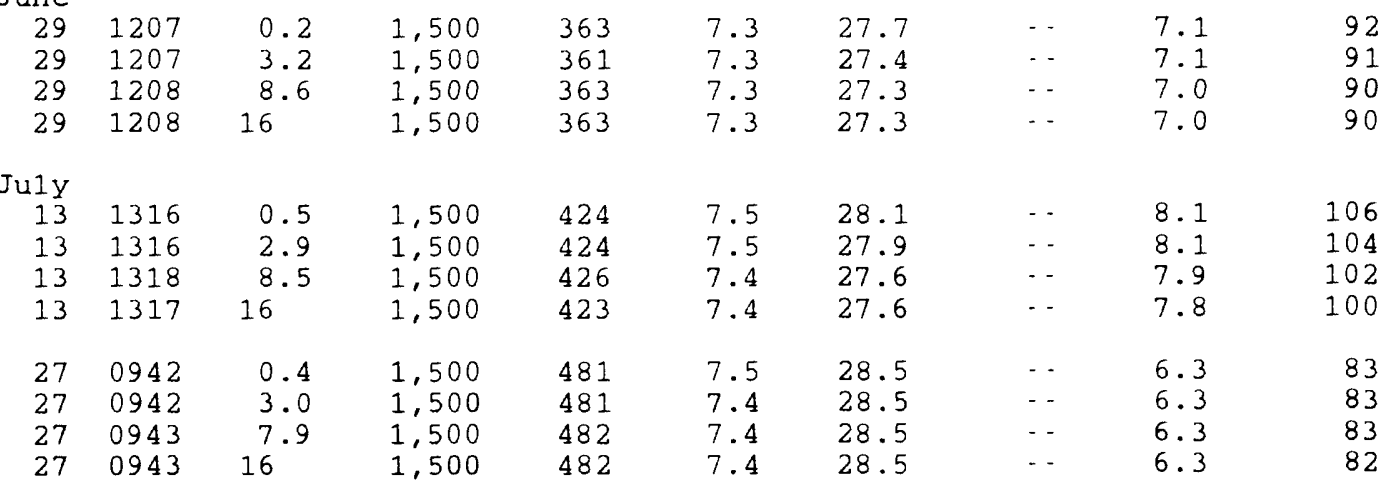

August

\begin{tabular}{|c|c|c|c|c|c|c|c|c|c|}
\hline 10 & 1401 & 1.1 & 1,500 & 429 & 7.6 & 28.6 & $\cdots$ & 7.2 & 94 \\
\hline 10 & 1401 & 3.0 & 1,500 & 422 & 7.5 & 27.8 & - & 6.6 & 85 \\
\hline 10 & 1402 & 8.6 & 1,500 & 418 & 7.5 & 27.6 & $\cdots$ & 6.6 & 86 \\
\hline 10 & 1402 & 16 & 1,500 & 393 & 7.5 & 26.2 & $\cdots$ & 6.2 & 79 \\
\hline 24 & 1630 & 0.4 & 1,500 & 532 & 7.7 & 31.3 & $\cdots$ & 8.4 & 116 \\
\hline 24 & 1631 & 3.1 & 1,500 & 532 & 7.5 & 30.3 & - & 7.8 & 106 \\
\hline 24 & 1632 & 8.1 & 1,500 & 533 & 7.3 & 29.8 & $\cdots$ & 6.7 & 90 \\
\hline 24 & 1631 & 15 & 1,500 & 528 & 7.3 & 29.4 & - & 6.1 & 82 \\
\hline
\end{tabular}

September

\begin{tabular}{|c|c|c|c|c|c|c|c|c|}
\hline $06 \quad 1217$ & 0.2 & 1,500 & 446 & 7.3 & 27.4 & -- & 7.6 & 96 \\
\hline 1217 & 2.8 & 1,500 & 544 & 7.2 & 27.2 & $\cdots$ & 7.3 & 93 \\
\hline 1218 & 7.6 & 1,500 & 544 & 7.2 & 27.2 & $\cdots$ & 7.1 & 91 \\
\hline 1219 & 15 & 1,500 & 544 & 7.2 & 27.2 & - & 7.1 & 91 \\
\hline 0952 & 1.2 & 1,500 & 664 & 7.3 & 23.5 & $\cdots$ & 6.6 & 78 \\
\hline 0953 & 3.7 & 1.500 & 664 & 7.3 & 23.6 & $\cdots$ & 7.1 & 85 \\
\hline
\end{tabular}


Table 9. Water-quality data for station 392419081255001, ohio River at river mile 170.8, main channel, June to October, 1995, Continued.

\begin{tabular}{|c|c|c|c|c|c|c|c|c|c|}
\hline Date & Time & $\begin{array}{l}\text { Sampling } \\
\text { depth } \\
(\mathrm{f} t)\end{array}$ & $\begin{array}{c}\text { Sample } \\
\text { location } \\
\text { (tt trom } \\
\text { left bank) }\end{array}$ & $\begin{array}{l}\text { Specific } \\
\text { conduct- } \\
\text { ance } \\
(\mu \mathrm{S} / \mathrm{cm})\end{array}$ & $\begin{array}{l}\mathrm{pH} \\
\text { (stan- } \\
\text { dard } \\
\text { undts) }\end{array}$ & $\begin{array}{c}\text { Temper- } \\
\text { ature, } \\
\text { water } \\
\left({ }^{\circ} \mathrm{C}\right)\end{array}$ & $\begin{array}{l}\text { Trans- } \\
\text { parency } \\
\text { (secchd } \\
\text { disk) } \\
\text { (ft) }\end{array}$ & $\begin{array}{l}\text { Dissolved } \\
\text { oxygen } \\
(\mathrm{mg} / \mathrm{L})\end{array}$ & $\begin{array}{c}\text { Dissolved } \\
\text { oxygen } \\
\text { (percent } \\
\text { satura- } \\
\text { tion) }\end{array}$ \\
\hline \multicolumn{10}{|c|}{ September } \\
\hline 20 & 1740 & 1.5 & 1,500 & 662 & 7.4 & 23.7 & $\cdots$ & 6.5 & 78 \\
\hline 20 & 1740 & 3.1 & 1,500 & 661 & 7.3 & 23.7 & - & 6.4 & 77 \\
\hline 20 & 1741 & 7.6 & 1,500 & 661 & 7.3 & 23.7 & $\ldots$ & 6.4 & 77 \\
\hline 20 & 1741 & 16 & 1,500 & 664 & 7.3 & 23.7 & -- & 6.4 & 77 \\
\hline \multicolumn{10}{|c|}{ October } \\
\hline 05 & 2009 & 0.7 & 1,500 & 554 & 7.4 & 21.9 & - & 6.8 & 79 \\
\hline 05 & 2009 & 3.2 & 1,500 & 552 & 7.3 & 22.0 & $\cdots$ & 6.8 & 79 \\
\hline 05 & 2010 & 8.4 & 1,500 & 555 & 7.3 & 22.0 & $\cdots$ & 6.8 & 79 \\
\hline 05 & 2010 & 17 & 1,500 & 569 & 7.3 & 22.0 & $\cdots$ & 6.7 & 78 \\
\hline 19 & 1034 & 1.1 & 1,500 & 552 & 7.4 & 19.2 & - & 7.8 & 85 \\
\hline 19 & 1034 & 3.2 & 1,500 & 552 & 7.4 & 19.1 & $\cdots$ & 7.8 & 85 \\
\hline 19 & 1035 & 7.5 & 1,500 & 552 & 7.4 & 19.2 & $\cdots$ & 7.4 & 81 \\
\hline 19 & 1035 & 14 & 1,500 & 552 & 7.4 & 19.1 & - & 7.9 & 87 \\
\hline
\end{tabular}


Table 10. Water-quality data for station 392411081255901, Ohio River at river mile 170.8, back channel, June to October, 1995.

[ft $=$ feet $; \mu \mathrm{S} / \mathrm{cm}=$ microsiemens per centımeter $;{ }^{\circ} \mathrm{C}=$ degrees Celsius; $\mathrm{mg} / \mathrm{L}=\mathrm{milligrams}$ per liter;.. = data not collected]

\begin{tabular}{|c|c|c|c|c|c|c|c|c|c|}
\hline Date & Time & $\begin{array}{l}\text { Sampling } \\
\text { depth } \\
\text { (ft) }\end{array}$ & $\begin{array}{c}\text { Sample } \\
\text { location } \\
\text { (ft trom } \\
\text { lett bank) }\end{array}$ & $\begin{array}{l}\text { Specific } \\
\text { conduct- } \\
\text { ance } \\
(\mu \mathrm{S} / \mathrm{cm})\end{array}$ & $\begin{array}{l}\mathrm{pH} \\
\text { (stan- } \\
\text { dard } \\
\text { units) }\end{array}$ & $\begin{array}{c}\text { Temper- } \\
\text { ature, } \\
\text { water } \\
\left({ }^{\circ} \mathrm{C}\right)\end{array}$ & $\begin{array}{l}\text { Trans- } \\
\text { parency } \\
\text { (Secchl } \\
\text { disk) } \\
\text { (ft) }\end{array}$ & $\begin{array}{l}\text { Dissolved } \\
\text { oxygen } \\
(\mathrm{mg} / \mathrm{L})\end{array}$ & $\begin{array}{c}\text { Dissolved } \\
\text { oxygen } \\
\text { (percent } \\
\text { satura- } \\
\text { tion) }\end{array}$ \\
\hline \multicolumn{10}{|l|}{ June } \\
\hline 29 & 1201 & 0.5 & 400 & 361 & 7.3 & 27.4 & $\cdots$ & 7.0 & 90 \\
\hline 29 & 1201 & 3.0 & 400 & 363 & 7.3 & 27.4 & $\cdots$ & 6.9 & 89 \\
\hline 29 & 1203 & 9.0 & 400 & 364 & 7.3 & 27.3 & $\cdots$ & 6.9 & 89 \\
\hline 29 & 1202 & 18 & 400 & 359 & 7.3 & 27.3 & $\cdots$ & 6.9 & 89 \\
\hline \multicolumn{10}{|l|}{ July } \\
\hline 13 & 1310 & 0.4 & 400 & 423 & 7.4 & 28.0 & $\cdots$ & 7.9 & 103 \\
\hline 13 & 1310 & 3.5 & 400 & 424 & 7.4 & 27.8 & $\cdots$ & 8.0 & 103 \\
\hline 13 & 1311 & 8.2 & 400 & 423 & 7.4 & 27.6 & $\cdots$ & 7.7 & 100 \\
\hline 13 & 1311 & 16 & 400 & 425 & 7.4 & 27.6 & $\cdots$ & 7.7 & 99 \\
\hline 27 & 0955 & 0.3 & 400 & 476 & 7.3 & 28.6 & $\cdots$ & 6.3 & 83 \\
\hline 27 & 0956 & 3.2 & 400 & 480 & 7.3 & 28.5 & $\cdots$ & 6.3 & 82 \\
\hline 27 & 0957 & 7.1 & 400 & 482 & 7.3 & 28.5 & $\cdots$ & 6.2 & 82 \\
\hline 27 & 0956 & 14 & 400 & 481 & 7.3 & 28.5 & $\cdots$ & 6.2 & 81 \\
\hline \multicolumn{10}{|c|}{ August } \\
\hline 10 & 1408 & 1.1 & 400 & 440 & 7.6 & 28.8 & $\cdots$ & 7.2 & 95 \\
\hline 10 & 1408 & 3.6 & 400 & 438 & 7.6 & 28.4 & $\cdots$ & 7.1 & 93 \\
\hline 10 & 1409 & 8.5 & 400 & 432 & 7.4 & 27.8 & $\cdots$ & 6.4 & 83 \\
\hline 10 & 1409 & 15 & 400 & 431 & 7.4 & 27.7 & $\cdots$ & 6.2 & 81 \\
\hline 24 & 1623 & 0.3 & 400 & 532 & 7.6 & 31.3 & $-\cdot$ & 8.1 & 111 \\
\hline 24 & 1623 & 3.2 & 400 & 532 & 7.5 & 31.0 & $-\cdot$ & 8.0 & 109 \\
\hline 24 & 1624 & 7.5 & 400 & 530 & 7.2 & 29.8 & $\cdots$ & 6.3 & 85 \\
\hline 24 & 1624 & 15 & 400 & 531 & 7.2 & 29.7 & $\cdots$ & 5.9 & 78 \\
\hline \multicolumn{10}{|c|}{ September } \\
\hline 06 & 1210 & 0.3 & 400 & 544 & 7.2 & 27.8 & $\cdots$ & 7.5 & 96 \\
\hline 06 & 1210 & 3.0 & 400 & 543 & 7.2 & 27.4 & $\cdots$ & 7.1 & 91 \\
\hline 06 & 1212 & 7.6 & 400 & 542 & 7.1 & 27.2 & -- & 6.8 & 87 \\
\hline 06 & 1211 & 15 & 400 & 539 & 7.1 & 27.2 & $\cdots$ & 6.8 & 86 \\
\hline 19 & 0959 & 1.1 & 400 & 664 & 7.2 & 23.6 & $\cdots$ & 6.2 & 74 \\
\hline 19 & 0959 & 3.2 & 400 & 666 & 7.2 & 23.6 & $\cdots$ & 6.0 & 71 \\
\hline 19 & 1001 & 7.3 & 400 & 667 & 7.2 & 23.6 & $\cdots$ & 6.0 & 72 \\
\hline 19 & 1000 & 15 & 400 & 663 & 7.2 & 23.5 & $\cdots$ & 6.0 & 72 \\
\hline
\end{tabular}


Table 10. Water-quality data for station 392411081255901, Ohio River at river mile 170.8, back channel, June to October, 1995, Continued.

[ft $=$ feet; $\mu \mathrm{s} / \mathrm{cm}=$ microsiemens per centimeter; ${ }^{\circ} \mathrm{C}=$ degrees Celsius; $\mathrm{mg} / \mathrm{L}=$ mlligrams per liter; .. = data not collected]

\begin{tabular}{|c|c|c|c|c|c|c|c|c|c|c|}
\hline Date & Time & $\begin{array}{l}\text { Sampling } \\
\text { depth } \\
\text { (ft) }\end{array}$ & $\begin{array}{r}\text { San } \\
\text { loca } \\
\text { let } \\
\text { left }\end{array}$ & $\begin{array}{l}\text { mple } \\
\text { ation } \\
\text { trom } \\
\text { bankl }\end{array}$ & $\begin{array}{l}\text { Specific } \\
\text { conduct - } \\
\text { ance } \\
(\mu \mathrm{S} / \mathrm{cm})\end{array}$ & $\begin{array}{l}\mathrm{pH} \\
\text { (stan- } \\
\text { dard } \\
\text { units) }\end{array}$ & $\begin{array}{c}\text { Temper- } \\
\text { ature, } \\
\text { water } \\
\left({ }^{\circ} \mathrm{C}\right)\end{array}$ & $\begin{array}{l}\text { Trans- } \\
\text { parency } \\
\text { (Secchl } \\
\text { disk) } \\
\text { (ft) }\end{array}$ & $\begin{array}{l}\text { Dissolved } \\
\text { oxygen } \\
(\mathrm{mg} / \mathrm{L})\end{array}$ & $\begin{array}{c}\text { Dissolved } \\
\text { oxygen } \\
\text { (percent } \\
\text { satura- } \\
\text { tion) }\end{array}$ \\
\hline \multicolumn{11}{|c|}{ September } \\
\hline 20 & 1829 & 1.5 & & 400 & 652 & 7.3 & 23.7 & $\cdots$ & 6.2 & 75 \\
\hline 20 & 1829 & 3.1 & & 400 & 663 & 7.3 & 23.7 & - & 6.2 & 75 \\
\hline 20 & 1831 & 8.4 & & 400 & 660 & 7.3 & 23.7 & $\cdots$ & 6.1 & 73 \\
\hline 20 & 1830 & 16 & & 400 & 660 & 7.3 & 23.7 & $\cdots$ & 6.1 & 74 \\
\hline 21 & 1033 & 0.5 & & 400 & 656 & 7.2 & 23.8 & $\cdots$ & 5.8 & 70 \\
\hline 21 & 1033 & 3.0 & & 400 & 658 & 7.2 & 23.8 & - & 5.9 & 70 \\
\hline 21 & 1034 & 8.9 & & 400 & 659 & 7.2 & 23.8 & - & 5.9 & 70 \\
\hline 21 & 1034 & 19 & & 400 & 655 & 7.2 & 23.8 & $\cdots$ & 5.9 & 71 \\
\hline \multicolumn{11}{|c|}{ October } \\
\hline 05 & 1650 & 0.6 & & 400 & 552 & 7.4 & 21.9 & $\cdots$ & 6.7 & 78 \\
\hline 05 & 1651 & 3.3 & & 400 & 556 & 7.3 & 21.9 & - & 6.7 & 77 \\
\hline 05 & 1652 & 9.0 & & 400 & 561 & 7.3 & 21.9 & $\cdots$ & 6.6 & 76 \\
\hline 05 & 1651 & 17 & & 400 & 551 & 7.3 & 21.9 & $-\cdot$ & 6.6 & 77 \\
\hline 19 & 1039 & 0.6 & & 400 & 552 & 7.4 & 19.3 & $\cdots$ & 7.6 & 84 \\
\hline 19 & 1040 & 3.4 & & 400 & 552 & 7.4 & 19.2 & $\cdots$ & 7.5 & 82 \\
\hline 19 & 1041 & 7.2 & & 400 & 551 & 7.4 & 19.1 & $\cdots$ & 7.2 & 79 \\
\hline 19 & 1040 & 16 & & 400 & 552 & 7.4 & 19.1 & $\cdots$ & 7.3 & 80 \\
\hline
\end{tabular}


Table 11. Water-quality data for station 392232081295601, Ohio River at river mile 175.5, main channel, June to October, 1995.

Ift $=$ feet; $\mu \mathrm{s} / \mathrm{cm}=$ microsiemens per centımeter $;{ }^{\circ} \mathrm{C}=$ degrees Celsius:

$\mathrm{mg} / \mathrm{L}=\mathrm{m}$ lligrams per liter; - = data not collected]

\begin{tabular}{|c|c|c|c|c|c|c|c|c|c|}
\hline Date & Time & $\begin{array}{l}\text { Sampling } \\
\text { depth } \\
\text { (Et) }\end{array}$ & $\begin{array}{c}\text { Sample } \\
\text { location } \\
\text { (tt from } \\
\text { left bank) }\end{array}$ & $\begin{array}{l}\text { Specleic } \\
\text { conduct - } \\
\text { ance } \\
(\mu \mathrm{S} / \mathrm{cm})\end{array}$ & $\begin{array}{l}\text { pH } \\
\text { (stan- } \\
\text { dard } \\
\text { units) }\end{array}$ & $\begin{array}{c}\text { Temper- } \\
\text { ature, } \\
\text { water } \\
\left({ }^{\circ} \mathrm{C}\right)\end{array}$ & $\begin{array}{c}\text { Trans - } \\
\text { parency } \\
\text { (Secchi } \\
\text { disk) } \\
(t t)\end{array}$ & $\begin{array}{l}\text { Dissolved } \\
\text { oxygen } \\
(\mathrm{mg} / \mathrm{L})\end{array}$ & $\begin{array}{c}\text { Dissolved } \\
\text { oxygen } \\
\text { (percent } \\
\text { satura- } \\
\text { tion) }\end{array}$ \\
\hline
\end{tabular}

\begin{tabular}{|c|c|c|c|c|c|c|c|c|c|}
\hline \multicolumn{10}{|l|}{ June } \\
\hline 29 & 1219 & 0.2 & 1,700 & 432 & 7.6 & 27.5 & $\cdots$ & 7.3 & 94 \\
\hline 29 & 1219 & 3.2 & 1,700 & 431 & 7.6 & 27.4 & $\cdots$ & 7.3 & 94 \\
\hline 29 & 1221 & 10 & 1,700 & 437 & 7.6 & 27.3 & $\cdots$ & 7.2 & 93 \\
\hline 29 & 1220 & 21 & 1,700 & 435 & 7.6 & 27.3 & $\cdots$ & 7.2 & 93 \\
\hline \multicolumn{10}{|l|}{ July } \\
\hline 13 & 1420 & 0.4 & 1,700 & 467 & 8.5 & 29.6 & $\cdots$ & 10.4 & 13 \\
\hline 13 & 1420 & 3.4 & 1,700 & 471 & 8.2 & 27.9 & $\cdots$ & 9.2 & 11 \\
\hline 13 & 1422 & 11 & 1,700 & 470 & 8.0 & 27.8 & $\cdots$ & 8.6 & 11 \\
\hline 13 & 1421 & 19 & 1,700 & 468 & 8.1 & 27.8 & $\cdots$ & 8.6 & 11 \\
\hline 14 & 1407 & 0.3 & 1,700 & 470 & 8.5 & 29.2 & $\cdots$ & 10.6 & 14 \\
\hline 14 & 1408 & 1.0 & 1,700 & 470 & 8.5 & 29.3 & - - & 10.9 & \\
\hline 14 & 1408 & 2.1 & 1,700 & 469 & 8.5 & 29.3 & 2.0 & 10.9 & \\
\hline 14 & 1409 & 3.0 & 1,700 & 471 & 8.4 & 29.0 & - & 10.5 & \\
\hline 14 & 1410 & 3.7 & 1,700 & 473 & 8.2 & 28.4 & $\cdots$ & 9.5 & \\
\hline 14 & 1411 & 5.1 & 1,700 & 473 & 8.2 & 28.3 & $\cdots$ & 9.5 & \\
\hline 14 & 1412 & 10 & 1,700 & 476 & 7.9 & 28.1 & $\cdots$ & 8.5 & 11 \\
\hline 14 & 1413 & 19 & 1,700 & 475 & 7.9 & 28.1 & $\cdots$ & 8.4 & 109 \\
\hline 27 & 1305 & 0.4 & 1,700 & 553 & 7.6 & 29.2 & $\cdots$ & 6.9 & \\
\hline 27 & 1305 & 3.1 & 1,700 & 553 & 7.6 & 29.1 & $\cdots$ & 6.9 & \\
\hline 27 & 1306 & 10 & 1,700 & 547 & 7.6 & 29.1 & $\cdots$ & 6.8 & \\
\hline 27 & 1306 & 20 & 1,700 & 546 & 7.6 & 29.0 & $\cdots$ & 6.8 & \\
\hline \multicolumn{10}{|c|}{ August } \\
\hline 10 & 1418 & 1.1 & 1,700 & 357 & 7.7 & 24.9 & $\cdots$ & 7.7 & \\
\hline 10 & 1418 & 3.8 & 1.700 & 354 & 7.7 & 23.9 & $\cdots$ & 7.7 & \\
\hline 10 & 1420 & 11 & 1,700 & 351 & 7.7 & 23.9 & $\cdots$ & 7.6 & 9 \\
\hline 10 & 1419 & 20 & 1,700 & 352 & 7.7 & 23.9 & $-\cdot$ & 7.6 & 9 \\
\hline 24 & 1144 & 0.5 & 1,700 & 513 & 7.8 & 29.8 & $\cdots$ & 8.0 & 107 \\
\hline 24 & 1145 & 3.4 & 1,700 & 514 & 7.7 & 29.2 & $\cdots$ & 7.5 & \\
\hline 24 & 1146 & 10 & 1,700 & 513 & 7.6 & 29.1 & $\cdots$ & 7.0 & \\
\hline 24 & 1145 & 20 & 1.700 & 512 & 7.6 & 29.0 & $\cdots$ & 7.1 & \\
\hline
\end{tabular}


Table 11. Water-quality data for station 392232081295601, Ohio River at river mile 175.5, main channel, June to October, 1995, Continued.

\begin{tabular}{|c|c|c|c|c|c|c|c|c|c|}
\hline Date & Time & $\begin{array}{l}\text { Sampling } \\
\text { depth } \\
\text { (ft) }\end{array}$ & $\begin{array}{c}\text { Sample } \\
\text { location } \\
\text { (tt from } \\
\text { left bank) }\end{array}$ & $\begin{array}{l}\text { Specific } \\
\text { conduct- } \\
\text { ance } \\
(\mu \mathrm{S} / \mathrm{cm})\end{array}$ & $\begin{array}{l}\mathrm{pH} \\
\text { (stan- } \\
\text { dard } \\
\text { units) }\end{array}$ & $\begin{array}{c}\text { Temper- } \\
\text { ature, } \\
\text { water } \\
\left({ }^{\circ} \mathrm{C}\right)\end{array}$ & $\begin{array}{l}\text { Trans- } \\
\text { parency } \\
\text { (Secchl } \\
\text { disk) } \\
\text { (ft) }\end{array}$ & $\begin{array}{l}\text { Dissolved } \\
\text { oxygen } \\
(\mathrm{mg} / \mathrm{L})\end{array}$ & $\begin{array}{c}\text { Dissolved } \\
\text { oxygen } \\
\text { (percent } \\
\text { satura- } \\
\text { tion) }\end{array}$ \\
\hline \multicolumn{10}{|c|}{ September } \\
\hline 06 & 1228 & 0.2 & 1,700 & 555 & 8.1 & 28.5 & $\ldots$ & 9.8 & 128 \\
\hline 06 & 1228 & 3.0 & 1,700 & 554 & 8.0 & 27.9 & $\cdots$ & 9.4 & 121 \\
\hline 06 & 1229 & 8.5 & 1,700 & 554 & 7.5 & 27.1 & -- & 7.5 & 95 \\
\hline 06 & 1229 & 18 & 1,700 & 554 & 7.5 & 27.0 & $\cdots$ & 7.4 & 94 \\
\hline 20 & 1107 & 0.2 & 1,700 & 684 & 7.6 & 23.5 & $\cdots$ & 6.9 & 83 \\
\hline 20 & 1108 & 2.3 & 1.700 & 685 & 7.6 & 23.5 & $\cdots$ & 7.1 & 84 \\
\hline 20 & 1110 & 10 & 1,700 & 685 & 7.6 & 23.5 & $\cdots$ & 7.0 & 83 \\
\hline 20 & 1109 & 20 & 1,700 & 685 & 7.6 & 23.5 & - & 7.0 & 84 \\
\hline \multicolumn{10}{|c|}{ October } \\
\hline 05 & 1632 & 0.7 & 1,700 & 601 & 7.5 & 21.7 & $\cdots$ & 6.7 & 78 \\
\hline 05 & 1632 & 3.3 & 1,700 & 596 & 7.4 & 21.7 & $\cdots$ & 6.6 & 76 \\
\hline 05 & 1633 & 7.9 & 1,700 & 605 & 7.4 & 21.7 & - & 6.6 & 76 \\
\hline 05 & 1633 & 16 & 1,700 & 608 & 7.4 & 21.7 & $-\cdot$ & 6.5 & 76 \\
\hline 19 & 1340 & 0.6 & 1,700 & 544 & 7.7 & 19.3 & - & 8.4 & 92 \\
\hline 19 & 1341 & 3.2 & 1,700 & 545 & 7.6 & 19.0 & $-\cdot$ & 8.2 & 89 \\
\hline 19 & 1342 & 9.3 & 1,700 & 545 & 7.6 & 18.4 & - & 7.8 & 84 \\
\hline 19 & 1341 & 18 & 1.700 & 542 & 7.6 & 18.4 & -- & 7.7 & 83 \\
\hline
\end{tabular}


Table 12. Water-quality data for station 392227081293701, Ohio River at river mile 175.5, back channel, June to October, 1995.

$\left[\mathrm{ft}=\mathrm{feet} ; \boldsymbol{\mu S} / \mathrm{cm}=\right.$ microsiemens per centimeter $;{ }^{\circ} \mathrm{C}=$ degrees Celsius: $\mathrm{mg} / \mathrm{L}=$ milligrams per liter;..$=$ data not collected]

\begin{tabular}{|c|c|c|c|c|c|c|c|c|c|}
\hline Date & Time & $\begin{array}{l}\text { Sampling } \\
\text { depth } \\
\text { (Et) }\end{array}$ & $\begin{array}{c}\text { Sample } \\
\text { location } \\
\text { ( } \mathrm{ft} \text { from } \\
\text { left bank) }\end{array}$ & $\begin{array}{l}\text { Specific } \\
\text { conduct- } \\
\text { ance } \\
(\mu \mathrm{S} / \mathrm{cm})\end{array}$ & $\begin{array}{l}\mathrm{pH} \\
\text { (stan- } \\
\text { dard } \\
\text { units) }\end{array}$ & $\begin{array}{c}\text { Temper- } \\
\text { ature, } \\
\text { water } \\
\left({ }^{\circ} \mathrm{C}\right)\end{array}$ & $\begin{array}{c}\text { Trans - } \\
\text { parency } \\
\text { (secchl } \\
\text { dIsk) } \\
\text { (tt) }\end{array}$ & $\begin{array}{l}\text { Dissolved } \\
\text { oxygen } \\
(\mathrm{mg} / \mathrm{L})\end{array}$ & $\begin{array}{c}\text { Dissolved } \\
\text { oxygen } \\
\text { (percent } \\
\text { satura- } \\
\text { tion) }\end{array}$ \\
\hline
\end{tabular}

\begin{tabular}{|c|c|c|c|c|c|c|c|c|c|}
\hline \multicolumn{10}{|l|}{ June } \\
\hline 29 & 1222 & 0.2 & 500 & 414 & 7.6 & 27.7 & $\cdots$ & 7.4 & 96 \\
\hline 29 & 1223 & 2.9 & 500 & 425 & 7.6 & 27.4 & $\cdots$ & 7.3 & 94 \\
\hline 29 & 1224 & 10 & 500 & 429 & 7.6 & 27.3 & $\cdots$ & 7.2 & 92 \\
\hline 29 & 1223 & 21 & 500 & 429 & 7.6 & 27.3 & $\cdots$ & 7.1 & 92 \\
\hline \multicolumn{10}{|l|}{ July } \\
\hline 13 & 1425 & 0.5 & 500 & 451 & 8.3 & 29.5 & $\cdots$ & 10.1 & 134 \\
\hline 13 & 1425 & 3.2 & 500 & 461 & 8.2 & 28.4 & $\cdots$ & 9.6 & 125 \\
\hline 13 & 1427 & 14 & 500 & 480 & 8.2 & 27.8 & $\cdots$ & 8.9 & 116 \\
\hline 13 & 1426 & 28 & 500 & 481 & 8.1 & 27.7 & $\cdots$ & 8.6 & 111 \\
\hline 27 & 1309 & 0.4 & 500 & 501 & 7.5 & 29.4 & $\cdots$ & 7.0 & 93 \\
\hline 27 & 1309 & 3.3 & 500 & 496 & 7.5 & 29.0 & $\cdots$ & 6.9 & 91 \\
\hline 27 & 1310 & 12 & 500 & 493 & 7.4 & 28.9 & $\cdots$ & 6.8 & 90 \\
\hline 27 & 1310 & 22 & 500 & 496 & 7.4 & 28.8 & $\cdots$ & 6.7 & 89 \\
\hline \multicolumn{10}{|c|}{ August } \\
\hline 10 & 1422 & 1.2 & 500 & 358 & 7.7 & 24.9 & $\cdots$ & 7.6 & 93 \\
\hline 10 & 1423 & 3.8 & 500 & 366 & 7.7 & 24.3 & $\cdots$ & 7.5 & 91 \\
\hline 10 & 1424 & 11 & 500 & 357 & 7.7 & 23.8 & $\cdots$ & 7.6 & 92 \\
\hline 10 & 1423 & 21 & 500 & 357 & 7.7 & 23.8 & $\cdots$ & 7.6 & 92 \\
\hline 24 & 1128 & 0.8 & 500 & 515 & 7.8 & 29.7 & $\cdots$ & 8.2 & 109 \\
\hline 24 & 1128 & 3.3 & 500 & 512 & 7.8 & 29.2 & $\cdots$ & 8.0 & 106 \\
\hline 24 & 1129 & 13 & 500 & 515 & 7.6 & 28.9 & $\cdots$ & 7.1 & 94 \\
\hline 24 & 1129 & 27 & 500 & 516 & 7.6 & 28.8 & $\cdots$ & 7.0 & 93 \\
\hline \multicolumn{10}{|c|}{ September } \\
\hline 20 & 1102 & 1.5 & 500 & 684 & 7.6 & 23.5 & $\cdots$ & 6.9 & 82 \\
\hline 20 & 1102 & 6.0 & 500 & 684 & 7.6 & 23.5 & -- & 7.0 & 83 \\
\hline 20 & 1104 & 10 & 500 & 684 & 7.6 & 23.5 & $\cdots$ & 6.9 & 82 \\
\hline 20 & 1103 & 20 & 500 & 684 & 7.6 & 23.5 & $\cdots$ & 6.7 & 80 \\
\hline \multicolumn{10}{|c|}{ October } \\
\hline 05 & 1627 & 1.1 & 500 & 605 & 7.4 & 21.7 & $\cdots$ & 6.6 & 76 \\
\hline 05 & 1627 & 3.4 & 500 & 609 & 7.4 & 21.7 & $\cdots$ & 6.5 & 75 \\
\hline 05 & 1628 & 12 & 500 & 615 & 7.4 & 21.6 & $\cdots$ & 6.3 & 73 \\
\hline 05 & 1628 & 23 & 500 & 618 & 7.4 & 21.7 & $\cdots$ & 6.4 & 74 \\
\hline
\end{tabular}


Table 12. Water-quality data for station 392227081293701, Ohio River at river mile 175.5, back channel, June to October, 1995, Continued.

\begin{tabular}{|c|c|c|c|c|c|c|c|c|c|}
\hline Date & Time & $\begin{array}{l}\text { Sampling } \\
\text { depth } \\
\text { (ft) }\end{array}$ & $\begin{array}{c}\text { Sample } \\
\text { location } \\
\text { (ft from } \\
\text { left bank) }\end{array}$ & $\begin{array}{l}\text { Specific } \\
\text { conduct- } \\
\text { ance } \\
(\mu \mathrm{S} / \mathrm{cm})\end{array}$ & $\begin{array}{l}\mathrm{pH} \\
\text { (stan- } \\
\text { dard } \\
\text { units) }\end{array}$ & $\begin{array}{c}\text { Temper- } \\
\text { ature, } \\
\text { water } \\
\left({ }^{\circ} \mathrm{C}\right)\end{array}$ & $\begin{array}{c}\text { Trans- } \\
\text { parency } \\
\text { (Secchi } \\
\text { disk) } \\
(t t)\end{array}$ & $\begin{array}{l}\text { Dissolved } \\
\text { oxygen } \\
(\mathrm{mg} / \mathrm{L})\end{array}$ & $\begin{array}{c}\text { Dissolved } \\
\text { oxygen } \\
\text { (percent } \\
\text { satura- } \\
\text { tion) }\end{array}$ \\
\hline \multicolumn{10}{|c|}{ October } \\
\hline 19 & 1344 & 1.2 & 500 & 545 & 7.6 & 19.0 & - & 8.3 & 90 \\
\hline 19 & 1345 & 3.0 & 500 & 543 & 7.6 & 18.6 & - & 8.2 & 88 \\
\hline 19 & 1346 & 10 & 500 & 544 & 7.6 & 18.3 & - & 7.8 & 84 \\
\hline 19 & 1345 & 21 & 500 & 545 & 7.5 & 18.2 & - - & 7.8 & 84 \\
\hline
\end{tabular}


Table 13. Water-quality data for station 392139081312801, Ohio River at river mile 177.2, main channe1, June to October, 1995.

[Et $=$ feet; $\mu \mathrm{s} / \mathrm{cm}=$ microsiemens per centimeter $;{ }^{\circ} \mathrm{C}=$ degrees Celsius; $\mathrm{mg} / \mathrm{L}=\mathrm{mili}$ igrams per liter; - - = data not collected]

\begin{tabular}{|c|c|c|c|c|c|c|c|c|c|}
\hline Date & Time & $\begin{array}{l}\text { Sampling } \\
\text { depth } \\
\text { (ft) }\end{array}$ & $\begin{array}{c}\text { Sample } \\
\text { location } \\
\text { (tt from } \\
\text { left bank) }\end{array}$ & $\begin{array}{l}\text { Specific } \\
\text { conduct } \\
\text { ance } \\
(\mu \mathrm{s} / \mathrm{cm})\end{array}$ & $\begin{array}{l}\mathrm{pH} \\
\text { (stan- } \\
\text { dard } \\
\text { units\} }\end{array}$ & $\begin{array}{c}\text { Tempez- } \\
\text { ature, } \\
\text { water } \\
\left({ }^{\circ} \mathrm{C}\right)\end{array}$ & $\begin{array}{c}\text { Trans- } \\
\text { parency } \\
\text { (Secchi } \\
\text { disk) } \\
\text { (tt) }\end{array}$ & $\begin{array}{l}\text { Dissolved } \\
\text { oxygen } \\
(\mathrm{mg} / \mathrm{L})\end{array}$ & $\begin{array}{c}\text { Dissolved } \\
\text { oxygen } \\
\text { (percent } \\
\text { satura- } \\
\text { tion) }\end{array}$ \\
\hline
\end{tabular}

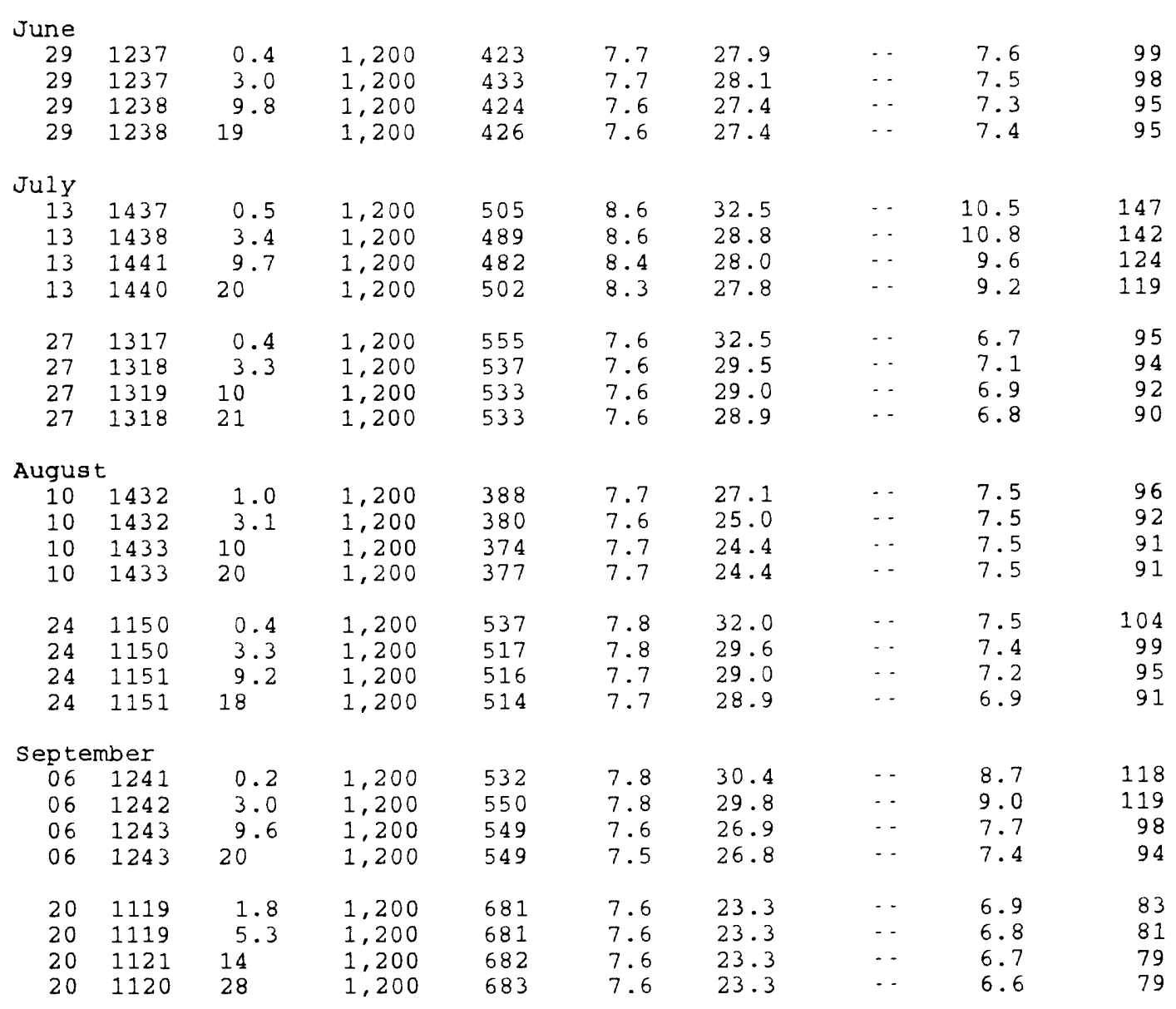


Table 13. Water-quality data for station 392139081312801, Ohio River at river mile 177.2, main channel, June to October, 1995, Continued.

[ft $=$ feet $; \mu \mathrm{S} / \mathrm{cm}=$ microsiemens per centimeter $;{ }^{\circ} \mathrm{C}=$ degrees Celsius; $\mathrm{mg} / \mathrm{L}=\mathrm{milligrams}$ per liter; $\cdots$ = data not collected]

\begin{tabular}{|c|c|c|c|c|c|c|c|c|c|}
\hline Date & Time & $\begin{array}{l}\text { Sampling } \\
\text { depth } \\
\text { (ft) }\end{array}$ & $\begin{array}{c}\text { Sample } \\
\text { location } \\
\text { (tt from } \\
\text { left bank) }\end{array}$ & $\begin{array}{l}\text { Specific } \\
\text { conduct- } \\
\text { ance } \\
(\mu \mathrm{S} / \mathrm{cm})\end{array}$ & $\begin{array}{l}\mathrm{pH} \\
\text { (stan- } \\
\text { dard } \\
\text { units) }\end{array}$ & $\begin{array}{c}\text { Temper- } \\
\text { ature, } \\
\text { water } \\
\left({ }^{\circ} \mathrm{C}\right)\end{array}$ & $\begin{array}{l}\text { Trans - } \\
\text { parency } \\
\text { (Secch1 } \\
\text { dısk) } \\
(f t)\end{array}$ & $\begin{array}{l}\text { Dissolved } \\
\text { oxygen } \\
\text { (mg/L) }\end{array}$ & $\begin{array}{c}\text { Dissolved } \\
\text { oxygen } \\
\text { (percent } \\
\text { satura- } \\
\text { tion) }\end{array}$ \\
\hline \multicolumn{10}{|c|}{ October } \\
\hline 05 & 1617 & 0.6 & 1,200 & 599 & 7.5 & 24.2 & $\cdots$ & 6.8 & 82 \\
\hline 05 & 1617 & 3.1 & 1,200 & 644 & 7.5 & 22.4 & $\cdots$ & 6.8 & 79 \\
\hline 05 & 1618 & 10 & 1,200 & 619 & 7.4 & 21.7 & - & 6.5 & 76 \\
\hline 05 & 1618 & 19 & 1,200 & 622 & 7.4 & 21.7 & - & 6.6 & 76 \\
\hline 19 & 1352 & 0.4 & 1,200 & 551 & 7.6 & 20.7 & $\cdots$ & 8.2 & 93 \\
\hline 19 & 1353 & 3.1 & 1,200 & 552 & 7.6 & 20.5 & - - & 8.1 & 91 \\
\hline 19 & 1354 & 9.7 & 1,200 & $54 \overline{5}$ & 7.6 & 18.4 & - - & 7.9 & 85 \\
\hline 19 & 1353 & 20 & 1,200 & 549 & 7.6 & 18.3 & - & 7.9 & 85 \\
\hline
\end{tabular}


Table 14. Water-quality data for station 392131081312301, Ohio River at river mile 177.2, back channel, June to October, 1995.

[ft $=$ feet $; \mu \mathrm{S} / \mathrm{cm}=$ microsiemens per centimeter $;{ }^{\circ} \mathrm{C}=$ degrees Celsius; $\mathrm{mg} / \mathrm{L}=$ milligrams per liter; . = data not collected]

\begin{tabular}{|c|c|c|c|c|c|c|c|c|c|}
\hline Date & Time & $\begin{array}{l}\text { Sampling } \\
\text { depth } \\
\text { (Et) }\end{array}$ & $\begin{array}{l}\text { Sample } \\
\text { location } \\
\text { (Et from } \\
\text { left bank) }\end{array}$ & $\begin{array}{l}\text { Specific } \\
\text { conduct- } \\
\text { ance } \\
(\mu \mathrm{S} / \mathrm{cm})\end{array}$ & $\begin{array}{l}\mathrm{pH} \\
\text { (stan- } \\
\text { dard } \\
\text { units) }\end{array}$ & $\begin{array}{l}\text { Temper- } \\
\text { ature, } \\
\text { water } \\
\left({ }^{\circ} \mathrm{C}\right)\end{array}$ & $\begin{array}{c}\text { Trans - } \\
\text { parency } \\
\text { (Secchi } \\
\text { disk) } \\
(t t)\end{array}$ & $\begin{array}{l}\text { Dissolved } \\
\text { oxygen } \\
(\mathrm{mg} / \mathrm{L})\end{array}$ & $\begin{array}{c}\text { Dissolved } \\
\text { oxygen } \\
\text { (percent } \\
\text { satura- } \\
\text { tion) }\end{array}$ \\
\hline
\end{tabular}

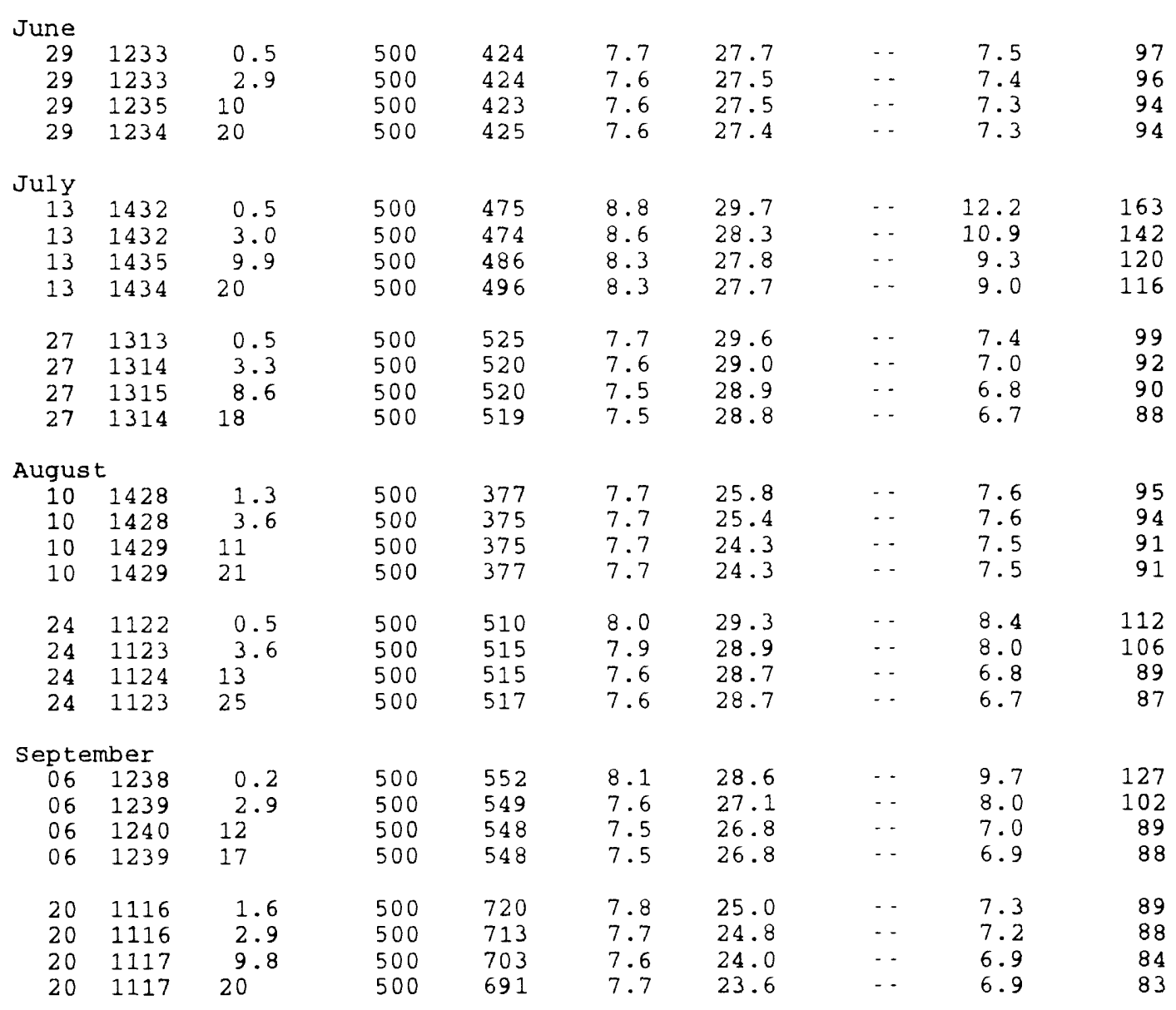


Table 14. Water-quality data for station 392131081312301, Ohio River at river mile 177.2, back channel, June to October, 1995, Continued.

[ft $=$ feet $; \mu \mathrm{S} / \mathrm{cm}=$ microsiemens per centımeter $;{ }^{\circ} \mathrm{C}=$ degrees Celsius; $\mathrm{mg} / \mathrm{L}=\mathrm{mili}$ igrams per liter; - - data not collected]

\begin{tabular}{|c|c|c|c|c|c|c|c|c|c|c|}
\hline Date & Time & $\begin{array}{l}\text { Sampling } \\
\text { depth } \\
\text { (ft) }\end{array}$ & $\begin{array}{r}\text { San } \\
100 a \\
1 \pm t \\
\text { lett }\end{array}$ & $\begin{array}{l}\text { mple } \\
\text { at ion } \\
\text { trom } \\
\text { bank) }\end{array}$ & $\begin{array}{l}\text { Specitic } \\
\text { conduct- } \\
\text { ance } \\
(\mu \mathrm{S} / \mathrm{cm})\end{array}$ & $\begin{array}{l}\mathrm{pH} \\
\text { (stan- } \\
\text { dard } \\
\text { units) }\end{array}$ & $\begin{array}{c}\text { Temper- } \\
\text { ature, } \\
\text { water } \\
\left({ }^{\circ} \mathrm{C}\right)\end{array}$ & $\begin{array}{c}\text { Trans - } \\
\text { parency } \\
\text { (Secchi } \\
\text { disk) } \\
\text { (tt) }\end{array}$ & $\begin{array}{l}\text { Dissolved } \\
\text { oxygen } \\
\text { (mg/L) }\end{array}$ & $\begin{array}{c}\text { Dissolved } \\
\text { oxygen } \\
\text { (percent } \\
\text { satura. } \\
\text { tion) }\end{array}$ \\
\hline \multicolumn{11}{|c|}{ October } \\
\hline 05 & 1620 & 0.7 & & 500 & 586 & 7.5 & 21.6 & $\cdots$ & 6.8 & 79 \\
\hline 05 & 1620 & 3.4 & & 500 & 612 & 7.4 & 21.6 & - & 6.6 & 77 \\
\hline 05 & 1622 & 14 & & 500 & 620 & 7.4 & 21.5 & $\cdots$ & 6.5 & 75 \\
\hline 05 & 1621 & 27 & & 500 & 622 & 7.4 & 21.5 & $\cdots$ & 6.6 & 76 \\
\hline 19 & 1349 & 0.5 & & 500 & 547 & 7.6 & 18.7 & $\cdots$ & 8.4 & 91 \\
\hline 19 & 1349 & 3.2 & & 500 & 548 & 7.6 & 18.3 & $\cdots$ & 8.1 & 87 \\
\hline 19 & 1350 & 7.5 & & 500 & 546 & 7.6 & 18.3 & $\cdots$ & 8.0 & 85 \\
\hline 19 & 1350 & 15 & & 500 & 547 & 7.5 & 18.1 & $\cdots$ & 7.6 & 81 \\
\hline
\end{tabular}


Table 15. Water-quality data for station 392042081330101, Ohio River at river mile 179.0, June to October, 1995.

ft $=$ feet; $\mu \mathrm{S} / \mathrm{cm}=$ microsiemens per centimeter; ${ }^{\circ} \mathrm{C}=$ degrees Celsius; $\mathrm{mg} / \mathrm{L}=\mathrm{mili}$ igrams per liter; . - = data not collected]

\begin{tabular}{|c|c|c|c|c|c|c|c|c|c|}
\hline Date & Time & $\begin{array}{l}\text { Sampling } \\
\text { depth } \\
\text { (Et) }\end{array}$ & $\begin{array}{c}\text { Sample } \\
\text { location } \\
\text { (Et from } \\
\text { left bank) }\end{array}$ & $\begin{array}{l}\text { Specitic } \\
\text { conduct - } \\
\text { ance } \\
(\mu \mathrm{s} / \mathrm{cm})\end{array}$ & $\begin{array}{l}\text { pH } \\
\text { (stan- } \\
\text { dard } \\
\text { units) }\end{array}$ & $\begin{array}{c}\text { Temper- } \\
\text { ature, } \\
\text { water } \\
\left({ }^{\circ} \mathrm{C}\right)\end{array}$ & $\begin{array}{l}\text { Trans- } \\
\text { parency } \\
\text { (secchi } \\
\text { disk) } \\
\text { (ft) }\end{array}$ & $\begin{array}{c}\text { Dissolved } \\
\text { oxygen } \\
(\mathrm{mg} / \mathrm{L})\end{array}$ & $\begin{array}{c}\text { Dissolved } \\
\text { oxygen } \\
\text { (percent } \\
\text { satura- } \\
\text { tion) }\end{array}$ \\
\hline
\end{tabular}

\begin{tabular}{|c|c|c|c|c|c|c|c|c|c|}
\hline $\begin{array}{r}\text { June } \\
29 \\
29 \\
29 \\
29\end{array}$ & $\begin{array}{l}1245 \\
1245 \\
1246 \\
1246\end{array}$ & $\begin{array}{r}0.2 \\
3.1 \\
11 \\
23\end{array}$ & $\begin{array}{l}800 \\
800 \\
800 \\
800\end{array}$ & $\begin{array}{l}431 \\
432 \\
436 \\
438\end{array}$ & $\begin{array}{l}7.6 \\
7.6 \\
7.6 \\
7.6\end{array}$ & $\begin{array}{l}27.5 \\
27.5 \\
27.5 \\
27.4\end{array}$ & $\begin{array}{l}\cdots \\
\cdots \\
\cdots\end{array}$ & $\begin{array}{l}7.4 \\
7.3 \\
7.3 \\
7.3\end{array}$ & $\begin{array}{l}95 \\
95 \\
94 \\
94\end{array}$ \\
\hline $\begin{array}{r}\text { July } \\
13 \\
13 \\
13 \\
13\end{array}$ & $\begin{array}{l}1447 \\
1448 \\
1452 \\
1450\end{array}$ & $\begin{array}{r}0.5 \\
3.5 \\
14 \\
19\end{array}$ & $\begin{array}{l}800 \\
800 \\
800 \\
800\end{array}$ & $\begin{array}{l}513 \\
512 \\
514 \\
515\end{array}$ & $\begin{array}{l}8.8 \\
8.6 \\
8.3 \\
8.2\end{array}$ & $\begin{array}{l}29.2 \\
28.2 \\
27.4 \\
27.4\end{array}$ & $\begin{array}{l}\cdots \\
\cdots \\
\cdots\end{array}$ & $\begin{array}{r}13.0 \\
10.9 \\
8.7 \\
8.6\end{array}$ & $\begin{array}{l}172 \\
143 \\
112 \\
110\end{array}$ \\
\hline $\begin{array}{l}27 \\
27 \\
27 \\
27\end{array}$ & $\begin{array}{l}1322 \\
1322 \\
1323 \\
1323\end{array}$ & $\begin{array}{r}0.3 \\
3.3 \\
13 \\
26\end{array}$ & $\begin{array}{l}800 \\
800 \\
800 \\
800\end{array}$ & $\begin{array}{l}530 \\
530 \\
531 \\
528\end{array}$ & $\begin{array}{l}7.7 \\
7.6 \\
7.5 \\
7.5\end{array}$ & $\begin{array}{l}30.0 \\
29.4 \\
29.0 \\
29.0\end{array}$ & $\begin{array}{l}\cdots \\
\cdots \\
\cdots\end{array}$ & $\begin{array}{l}7.5 \\
7.4 \\
6.7 \\
6.7\end{array}$ & $\begin{array}{r}100 \\
98 \\
89 \\
89\end{array}$ \\
\hline $\begin{array}{r}\text { Augu } \\
10 \\
10 \\
10 \\
10\end{array}$ & $\begin{array}{l}1438 \\
1438 \\
1440 \\
1439\end{array}$ & $\begin{array}{r}1.1 \\
3.8 \\
11 \\
21\end{array}$ & $\begin{array}{l}800 \\
800 \\
800 \\
800\end{array}$ & $\begin{array}{l}383 \\
379 \\
377 \\
381\end{array}$ & $\begin{array}{l}7.7 \\
7.7 \\
7.7 \\
7.7\end{array}$ & $\begin{array}{l}26.1 \\
24.7 \\
24.6 \\
24.6\end{array}$ & $\begin{array}{l}\cdots \\
\cdots \\
\cdots\end{array}$ & $\begin{array}{l}7.5 \\
7.4 \\
7.3 \\
7.4\end{array}$ & $\begin{array}{l}95 \\
91 \\
90 \\
90\end{array}$ \\
\hline $\begin{array}{l}24 \\
24 \\
24 \\
24\end{array}$ & $\begin{array}{l}1117 \\
1117 \\
1118 \\
1118\end{array}$ & $\begin{array}{l}0.4 \\
3.4 \\
13 \\
26\end{array}$ & $\begin{array}{l}800 \\
800 \\
800 \\
800\end{array}$ & $\begin{array}{l}523 \\
524 \\
520 \\
521\end{array}$ & $\begin{array}{l}7.8 \\
7.7 \\
7.7 \\
7.7\end{array}$ & $\begin{array}{l}29.4 \\
29.1 \\
29.0 \\
28.9\end{array}$ & $\begin{array}{l}\cdots \\
\cdots \\
\cdots\end{array}$ & $\begin{array}{l}7.7 \\
7.5 \\
6.8 \\
5.8\end{array}$ & $\begin{array}{r}102 \\
99 \\
90 \\
89\end{array}$ \\
\hline $\begin{array}{r}\text { Septe } \\
06 \\
06 \\
06 \\
06\end{array}$ & $\begin{array}{l}\text { ber } \\
1250 \\
1250 \\
1252 \\
1251\end{array}$ & $\begin{array}{r}0.3 \\
2.9 \\
9.7 \\
20\end{array}$ & $\begin{array}{l}800 \\
800 \\
800 \\
800\end{array}$ & $\begin{array}{l}552 \\
554 \\
553 \\
554\end{array}$ & $\begin{array}{l}8.3 \\
8.3 \\
7.7 \\
7.7\end{array}$ & $\begin{array}{l}28.7 \\
28.4 \\
27.6 \\
27.6\end{array}$ & $\begin{array}{l}\cdots \\
\cdots \\
\cdots\end{array}$ & $\begin{array}{r}10.7 \\
10.7 \\
8.2 \\
7.9\end{array}$ & $\begin{array}{l}139 \\
139 \\
105 \\
102\end{array}$ \\
\hline $\begin{array}{l}20 \\
20 \\
20 \\
20\end{array}$ & $\begin{array}{l}1126 \\
1126 \\
1128 \\
1127\end{array}$ & $\begin{array}{l}1.0 \\
3.4 \\
13 \\
25\end{array}$ & $\begin{array}{l}800 \\
800 \\
800 \\
800\end{array}$ & $\begin{array}{l}690 \\
688 \\
690 \\
692\end{array}$ & $\begin{array}{l}7.6 \\
7.6 \\
7.6 \\
7.6\end{array}$ & $\begin{array}{l}23.8 \\
23.8 \\
23.8 \\
23.8\end{array}$ & $\begin{array}{l}\cdots \\
\cdots \\
\cdots\end{array}$ & $\begin{array}{l}6.7 \\
6.8 \\
6.8 \\
6.8\end{array}$ & $\begin{array}{l}81 \\
81 \\
81 \\
81\end{array}$ \\
\hline
\end{tabular}


Table 15. Water-quality data for station 392042081330101, Ohio River at river mile 179.0, June to October, 1995, Continued.

$\left[t t=\right.$ feet $; \mu \mathrm{s} / \mathrm{cm}=$ microsiemens per centimeter; ${ }^{\circ} \mathrm{C}=$ degrees Celsius;

$\mathrm{mg} / \mathrm{L}=\mathrm{milligrams}$ per liter; $\cdots$ = data not collected]

\begin{tabular}{|c|c|c|c|c|c|c|c|c|c|}
\hline Date & Time & $\begin{array}{l}\text { Sampling } \\
\text { depth } \\
(\mathfrak{f t})\end{array}$ & $\begin{array}{c}\text { Sample } \\
\text { location } \\
\text { (ft from } \\
\text { left bank) }\end{array}$ & $\begin{array}{l}\text { Specific } \\
\text { conduct. } \\
\text { ance } \\
(\mu \mathrm{S} / \mathrm{cm})\end{array}$ & $\begin{array}{l}\mathrm{pH} \\
\text { (stan- } \\
\text { dard } \\
\text { units) }\end{array}$ & $\begin{array}{c}\text { Temper- } \\
\text { ature, } \\
\text { water } \\
\left({ }^{\circ} \mathrm{C}\right)\end{array}$ & $\begin{array}{l}\text { Trans- } \\
\text { parency } \\
\text { (Secchl } \\
\text { disk) } \\
(\mathrm{ft})\end{array}$ & $\begin{array}{l}\text { Dissolved } \\
\text { oxygen } \\
\text { (mg/L) }\end{array}$ & $\begin{array}{c}\text { Dissolved } \\
\text { oxygen } \\
\text { (percent } \\
\text { satura- } \\
\text { tron) }\end{array}$ \\
\hline \multicolumn{10}{|c|}{ October } \\
\hline 05 & 1609 & 0.2 & 800 & 614 & 7.5 & 21.8 & $\cdots$ & 7.1 & 83 \\
\hline 05 & 1609 & 2.7 & 800 & 617 & 7.5 & 21.8 & - - & 7.0 & 81 \\
\hline 05 & 1611 & 10 & 800 & 621 & 7.4 & 21.7 & $\cdots$ & 7.0 & 81 \\
\hline 05 & 1610 & 20 & 800 & 624 & 7.4 & 21.7 & $-\cdot$ & 6.9 & 80 \\
\hline 19 & 1359 & 0.5 & 800 & 553 & 7.6 & 19.6 & $\cdots$ & 8.1 & 90 \\
\hline 19 & 1359 & 3.2 & 800 & 551 & 7.5 & 19.3 & $\cdots$ & 7.8 & 86 \\
\hline 19 & 1401 & 12 & 800 & 542 & 7.5 & 18.7 & $\cdots$ & 7.6 & 83 \\
\hline 19 & 1400 & 25 & 800 & 546 & 7.5 & 18.7 & $\cdots$ & 7.5 & 82 \\
\hline
\end{tabular}


Table 16. Water-quality data for station 391822081334701, Ohio River at river mile 181.8, June to October, 1995.

[ft $=$ feet $; \mu \mathrm{s} / \mathrm{cm}=$ microsiemens per centimeter; ${ }^{\circ} \mathrm{C}=$ degrees Celsius; $\mathrm{mg} / \mathrm{L}=$ milligrams per liter; - - = data not collected]

\begin{tabular}{|c|c|c|c|c|c|c|c|c|c|}
\hline Date & Trme & $\begin{array}{l}\text { Sampling } \\
\text { depth } \\
\text { (ft) }\end{array}$ & $\begin{array}{c}\text { Sample } \\
\text { location } \\
\text { (ft Erom } \\
\text { left bank) }\end{array}$ & $\begin{array}{l}\text { Specific } \\
\text { conduct- } \\
\text { ance } \\
(\mu \mathrm{s} / \mathrm{cm})\end{array}$ & $\begin{array}{l}\text { pH } \\
\text { (stan- } \\
\text { dard } \\
\text { units) }\end{array}$ & $\begin{array}{c}\text { Temper- } \\
\text { ature, } \\
\text { water } \\
\left({ }^{\circ} \mathrm{C}\right)\end{array}$ & $\begin{array}{c}\text { Trans- } \\
\text { parency } \\
\text { (secchl } \\
\text { dlsk) } \\
(\mathrm{tt})\end{array}$ & $\begin{array}{c}\text { Dissolved } \\
\text { oxygen } \\
\text { (mg/L) }\end{array}$ & $\begin{array}{c}\text { Dissolved } \\
\text { oxygen } \\
\text { (percent } \\
\text { satura. } \\
\text { tion) }\end{array}$ \\
\hline
\end{tabular}

June

$\begin{array}{rrrrr}29 & 1337 & 0.4 & 500 & 42 \\ 29 & 1337 & 3.1 & 500 & 42 \\ 29 & 1338 & 11 & 500 & 42 \\ 29 & 1338 & 24 & 500 & 43\end{array}$

July

$\begin{array}{lllll}13 & 1459 & 0.4 & 500 & 49\end{array}$

$13 \quad 1500 \quad 3.0 \quad 500 \quad 49$

$\begin{array}{lllll}13 & 1501 & 12 & 500 & 492\end{array}$

$13 \quad 1501 \quad 23 \quad 500 \quad 493$

$428 \quad 7.7$

$\begin{array}{llll}27 & 1329 & 0.4 & 500\end{array}$

$27 \quad 1329 \quad 3.4$

500

$27 \quad 1330 \quad 12$

$27 \quad 1330 \quad 24$

500

547

547

542

7.6

27.7

27.6

$\begin{array}{ll}7.6 & 27.5 \\ 7.6 & 27.5\end{array}$

$\begin{array}{ll}\cdots & 7.6 \\ \cdots & 7.6\end{array}$

- 7.3

. $\quad 7.3$

98

428
433

27.5

500

542

$8.6 \quad 29.8$

- 11.7

157

$8.5 \quad 29.0$

8.128 .0

- $\quad 11.1$

147

$8.0 \quad 27.9$

8.9

116

August

$10 \quad 1446$

1.

$10 \quad 1446$

$10 \quad 1447$

3.6

500
500

500
500

7.7

30.2

.-

113

$10 \quad 1447 \quad 23$

500

424

7.7

29.7

- 7.6

103

$24 \quad 1108$

23

422
422

7.6

29.3

7.6

101

.
$\ldots$

- 6.9

93

$24 \quad 1109$

0.8

500

500

500

7.6

29.3

91

$24 \quad 1110 \quad 11$

$24 \quad 1109 \quad 23$

500

7.6

26.7

-

. 6.9

88

7.6

26.1

25.8

6.9

7.6

25.8

$-$

6.9

87

548

551

8.2

29.6

8.0

29.1

$7.9 \quad 29.0$

․ 6.9

86

september

061300

061300

0.2

500

553

7.9

29.0

․ 8.3

8.3
7.6

86

$06 \quad 1302$

2.9

500

551

552

552

7.8

28.1

7.7

27.8

$\begin{array}{ll}7.6 & 27.6\end{array}$

27.6

7.3

111

$06 \quad 1301 \quad 23$

500

552

7.6

23.7

500

674

7.6

23.7
23.7

500

676

7.6

23.8

674

7.6

23.8

7.2

96

95

$\begin{array}{lll}20 & 1137 & 23\end{array}$

500

676

7.6

$-$

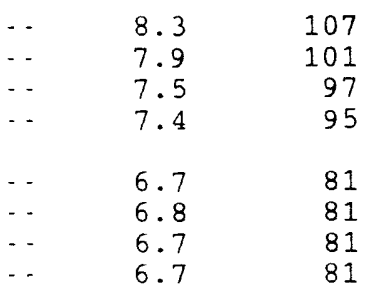


Table 16. Water-quality data for station 391822081334701, Ohio River at river mile 181.8, June to October, 1995, Continued.

$\left[\mathrm{ft}=\mathrm{feet} ; \mu \mathrm{s} / \mathrm{cm}=\right.$ microsiemens per centimeter $;{ }^{\circ} \mathrm{C}=$ degrees Celsius; $\mathrm{mg} / \mathrm{L}=\mathrm{milligrams}$ per liter: $\cdot$ = data not collected]

\begin{tabular}{|c|c|c|c|c|c|c|c|c|c|}
\hline Date & Time & $\begin{array}{l}\text { Sampling } \\
\text { depth } \\
\text { (ft) }\end{array}$ & $\begin{array}{l}\text { Sample } \\
\text { location } \\
\text { (tt trom } \\
\text { left bank) }\end{array}$ & $\begin{array}{l}\text { Specific } \\
\text { conduct- } \\
\text { ance } \\
(\mu \mathrm{s} / \mathrm{cm})\end{array}$ & $\begin{array}{l}\text { pH } \\
\text { (stan- } \\
\text { dard } \\
\text { units) }\end{array}$ & $\begin{array}{c}\text { Temper- } \\
\text { ature, } \\
\text { water } \\
\left({ }^{\circ} \mathrm{C}\right)\end{array}$ & $\begin{array}{l}\text { Trans - } \\
\text { parency } \\
\text { (Secchl } \\
\text { disk) } \\
\text { (tt) }\end{array}$ & $\begin{array}{c}\text { Dissolved } \\
\text { oxygen } \\
\text { (mg/L) }\end{array}$ & $\begin{array}{c}\text { Dissolved } \\
\text { oxygen } \\
\text { (percent } \\
\text { satura- } \\
\text { tron) }\end{array}$ \\
\hline
\end{tabular}

October

\begin{tabular}{|c|c|c|c|c|c|c|c|c|}
\hline $05 \quad 1559$ & 0.3 & 500 & 612 & 7.5 & 21.7 & $\cdots$ & 6.7 & 78 \\
\hline $05 \quad 1600$ & 2.9 & 500 & 619 & 7.5 & 21.7 & $\cdots$ & 6.7 & 77 \\
\hline 1601 & 11 & 500 & 626 & 7.5 & 21.7 & $\cdots$ & 6.7 & 78 \\
\hline 1600 & 23 & 500 & 622 & 7.5 & 21.7 & $\cdots$ & 6.7 & 77 \\
\hline 1407 & 0.6 & 500 & 557 & 7.6 & 19.9 & $\cdots$ & 8.2 & 90 \\
\hline 1407 & 3.3 & 500 & 555 & 7.5 & 19.0 & $\cdots$ & 7.8 & 85 \\
\hline 1408 & 11 & 500 & 553 & 7.5 & 18.8 & -- & 7.7 & 84 \\
\hline 1408 & 23 & 500 & 554 & 7.5 & 18.7 & - & 7.7 & 84 \\
\hline
\end{tabular}


Table 17. Water-quality data for station 391720081334701, Ohio River at river mile 183.0, June to October, 1995.

$\left[\mathrm{Et}=\mathrm{feet} ; \mu \mathrm{s} / \mathrm{cm}=\right.$ microsiemens per centimeter; ${ }^{\circ} \mathrm{C}=$ degrees celsius;

$\mathrm{mg} / \mathrm{L}=$ milligrams per liter: - = data not collected]

\begin{tabular}{|c|c|c|c|c|c|c|c|c|c|}
\hline Date & Time & $\begin{array}{l}\text { Sampling } \\
\text { depth } \\
\text { (ft) }\end{array}$ & $\begin{array}{c}\text { Sample } \\
\text { location } \\
\text { (Et from } \\
\text { left bank) }\end{array}$ & $\begin{array}{l}\text { Specific } \\
\text { conduct- } \\
\text { ance } \\
(\mu \mathrm{S} / \mathrm{cm})\end{array}$ & $\begin{array}{l}\mathrm{pH} \\
\text { (stan- } \\
\text { dard } \\
\text { units) }\end{array}$ & $\begin{array}{c}\text { Temper- } \\
\text { ature, } \\
\text { water } \\
\left({ }^{\circ} \mathrm{C}\right)\end{array}$ & $\begin{array}{c}\text { Trans- } \\
\text { parency } \\
\text { (secchi } \\
\text { disk) } \\
\text { (it) }\end{array}$ & $\begin{array}{l}\text { Dissolved } \\
\text { oxygen } \\
(\mathrm{mg} / \mathrm{L})\end{array}$ & $\begin{array}{c}\text { Dissolved } \\
\text { oxygen } \\
\text { (percent } \\
\text { satura- } \\
\text { tion) }\end{array}$ \\
\hline
\end{tabular}

\begin{tabular}{|c|c|c|c|c|c|c|c|c|c|}
\hline June & & & & & & & & & \\
\hline 29 & 0458 & 0.2 & 300 & 401 & 7.5 & 27.3 & $\cdots$ & 7.1 & 92 \\
\hline 29 & 0458 & 3.1 & 300 & 402 & 7.5 & 27.3 & $\cdots$ & 7.1 & 92 \\
\hline 29 & 0459 & 5.3 & 300 & 398 & 7.5 & 27.3 & $\cdots$ & 7.1 & 92 \\
\hline 29 & 0459 & 10 & 300 & 397 & 7.4 & 27.3 & $\cdots$ & 7.1 & 91 \\
\hline 29 & 0500 & 15 & 300 & 398 & 7.4 & 27.3 & $\cdots$ & 7.1 & 91 \\
\hline 29 & 0500 & 20 & 300 & 397 & 7.4 & 27.3 & $\cdots$ & 7.0 & 91 \\
\hline 29 & 0501 & 23 & 300 & 389 & 7.4 & 27.3 & $\cdots$ & 7.0 & 90 \\
\hline 29 & 0447 & 0.2 & 700 & 433 & 7.7 & 27.5 & $\cdots$ & 7.2 & 93 \\
\hline 29 & 0447 & 2.9 & 700 & 433 & 7.6 & 27.5 & $\cdots$ & 7.2 & 93 \\
\hline 29 & 0448 & 5.3 & 700 & 432 & 7.6 & 27.5 & $\cdots$ & 7.2 & 93 \\
\hline 29 & 0448 & 9.9 & 700 & 434 & 7.6 & 27.5 & $\cdots$ & 7.2 & 93 \\
\hline 29 & 0449 & 15 & 700 & 435 & 7.6 & 27.5 & $\cdots$ & 7.2 & 93 \\
\hline 29 & 0449 & 20 & 700 & 440 & 7.6 & 27.5 & $\cdots$ & 7.2 & 93 \\
\hline 29 & 0450 & 25 & 700 & 438 & 7.6 & 27.5 & $\cdots$ & 7.2 & 93 \\
\hline 29 & 0450 & 26 & 700 & 435 & 7.7 & 27.5 & $\cdots$ & 7.2 & 93 \\
\hline 29 & 0452 & 0.4 & 1,000 & 469 & 7.7 & 27.5 & $\cdots$ & 7.0 & 90 \\
\hline 29 & 0452 & 3.2 & 1,000 & 468 & 7.7 & 27.6 & $\cdots$ & 7.0 & 90 \\
\hline 29 & 0453 & 5.4 & 1,000 & 470 & 7.7 & 27.6 & $\cdots$ & 7.0 & 91 \\
\hline 29 & 0453 & 10 & 1,000 & 470 & 7.7 & 27.6 & $\cdots$ & 7.0 & 90 \\
\hline 29 & 0454 & 15 & 1,000 & 473 & 7.7 & 27.6 & $\cdots$ & 7.0 & 90 \\
\hline 29 & 0454 & 20 & 1.000 & 470 & 7.7 & 27.6 & $\cdots$ & 7.0 & 90 \\
\hline 29 & 1355 & 0.3 & 300 & 400 & 7.5 & 27.8 & $\cdots$ & 7.6 & 98 \\
\hline 29 & 1355 & 3.2 & 300 & 400 & 7.5 & 27.7 & $\cdots$ & 7.6 & 98 \\
\hline 29 & 1356 & 5.3 & 300 & 401 & 7.5 & 27.6 & $\cdots$ & 7.4 & 95 \\
\hline 29 & 1356 & 9.6 & 300 & 406 & 7.5 & 27.5 & $\cdots$ & 7.3 & 94 \\
\hline 29 & 1357 & 15 & 300 & 409 & 7.5 & 27.5 & $\cdots$ & 7.3 & 94 \\
\hline 29 & 1357 & 18 & 300 & 405 & 7.5 & 27.5 & $\cdots$ & 7.3 & 94 \\
\hline 29 & 1349 & 0.4 & 700 & 428 & 7.7 & 27.8 & $\cdots$ & 7.4 & 96 \\
\hline 29 & 1349 & 2.9 & 700 & 420 & 7.7 & 27.9 & $\cdots$ & 7.8 & 102 \\
\hline 29 & 1350 & 5.1 & 700 & 428 & 7.7 & 27.7 & $\cdots$ & 7.6 & 99 \\
\hline 29 & 1350 & 9.6 & 700 & 436 & 7.6 & 27.5 & $\cdots$ & 7.4 & 95 \\
\hline 29 & 1351 & 15 & 700 & 437 & 7.6 & 27.5 & - & 7.3 & 9 \\
\hline 29 & 1352 & 20 & 700 & 434 & 7.6 & 27.5 & $\cdots$ & 7.3 & 4 \\
\hline 29 & 1352 & 26 & 700 & 434 & 7.6 & 27.5 & $\cdots$ & 7.3 & 94 \\
\hline 29 & 1353 & 28 & 700 & 432 & 7.6 & 27.5 & $\cdots$ & 7.3 & 94 \\
\hline
\end{tabular}


Table 17. Water-quality data for station 391720081334701, Ohio River at river mile 183.0, June to October, 1995, Continued.

[Et $=$ feet $; \mu \mathrm{S} / \mathrm{cm}=$ microsiemens per centimeter $;{ }^{\circ} \mathrm{C}=$ degrees Celsius:

$\mathrm{mg} / \mathrm{L}=\mathrm{milligrams}$ per liter; $\cdots$ = data not collected]

\begin{tabular}{|c|c|c|c|c|c|c|c|c|c|}
\hline Date & Time & $\begin{array}{l}\text { Sampling } \\
\text { depth } \\
\text { (ft) }\end{array}$ & $\begin{array}{c}\text { Sample } \\
\text { location } \\
\text { (ft trom } \\
\text { left bank) }\end{array}$ & $\begin{array}{l}\text { Specific } \\
\text { conduct. } \\
\text { ance } \\
(\mu \mathrm{s} / \mathrm{cm})\end{array}$ & $\begin{array}{l}\mathrm{pH} \\
\text { (stan- } \\
\text { dard } \\
\text { units) }\end{array}$ & $\begin{array}{c}\text { Temper- } \\
\text { ature, } \\
\text { water } \\
\left({ }^{\circ} \mathrm{C}\right)\end{array}$ & $\begin{array}{l}\text { Trans- } \\
\text { parency } \\
\text { (secchl } \\
\text { disk) } \\
\text { ( } \mathrm{Et})\end{array}$ & $\begin{array}{l}\text { Dissolved } \\
\text { oxygen } \\
(\mathrm{mg} / \mathrm{L})\end{array}$ & $\begin{array}{r}\text { Dissolv } \\
\text { oxyge } \\
\text { (perce } \\
\text { satur } \\
\text { tion) }\end{array}$ \\
\hline \multicolumn{10}{|l|}{ June } \\
\hline 29 & 1345 & 0.3 & 1,000 & 459 & 7.9 & 28.2 & - & 8.0 & 104 \\
\hline 29 & 1345 & 2.9 & 1,000 & 460 & 7.9 & 28.2 & $\cdots$ & 8.1 & 105 \\
\hline 29 & 1346 & 4.8 & 1,000 & 459 & 7.8 & 27.8 & $\cdots$ & 7.9 & 103 \\
\hline 29 & 1346 & 10 & 1,000 & 460 & 7.8 & 27.7 & $\cdots$ & 7.5 & 97 \\
\hline 29 & 1347 & 17 & 1,000 & 460 & 7.7 & 27.7 & - & 7.5 & 97 \\
\hline 29 & 1347 & 19 & 1,000 & 460 & 7.7 & 27.7 & -- & 7.4 & 95 \\
\hline \multicolumn{10}{|l|}{ Ju1y } \\
\hline 13 & 0532 & 0.6 & 300 & 495 & 8.0 & 27.5 & $\cdots$ & 8.2 & 106 \\
\hline 13 & 0532 & 3.7 & 300 & 495 & 8.0 & 27.5 & - & 8.2 & 106 \\
\hline 13 & 0533 & 5.3 & 300 & 494 & 8.0 & 27.5 & - & 8.2 & 106 \\
\hline 13 & 0533 & 10 & 300 & 495 & 8.0 & 27.6 & $\cdots$ & 8.2 & 106 \\
\hline 13 & 0534 & 15 & 300 & 496 & 8.0 & 27.6 & $\cdots$ & 8.2 & 106 \\
\hline 13 & 0534 & 20 & 300 & 495 & 7.9 & 27.5 & $\cdots$ & 7.9 & 102 \\
\hline 13 & 0535 & 25 & 300 & 492 & 7.8 & 27.3 & -- & 7.3 & 94 \\
\hline 13 & 0535 & 27 & 300 & 495 & 7.7 & 27.3 & -- & 7.3 & 94 \\
\hline 13 & 0527 & 0.5 & 700 & 496 & 7.9 & 27.5 & $\cdots$ & 8.1 & 105 \\
\hline 13 & 0527 & 3.3 & 700 & 496 & 7.9 & 27.5 & -- & 8.1 & 105 \\
\hline 13 & 0528 & 5.0 & 700 & 495 & 7.9 & 27.5 & - & 8.1 & 105 \\
\hline 13 & 0528 & 9.9 & 700 & 496 & 7.9 & 27.5 & $-\cdot$ & 8.2 & 105 \\
\hline 13 & 0529 & 15 & 700 & 496 & 7.9 & 27.5 & $\cdots$ & 8.1 & 104 \\
\hline 13 & 0529 & 20 & 700 & 496 & 7.9 & 27.5 & - & 8.0 & 103 \\
\hline 13 & 0530 & 25 & 700 & 494 & 7.8 & 27.4 & $\cdots$ & 7.4 & 96 \\
\hline 13 & 0530 & 26 & 700 & 494 & 7.8 & 27.4 & $\cdots$ & 7.4 & 95 \\
\hline 13 & 0522 & 0.5 & 1,000 & 496 & 8.0 & 27.6 & $\cdots$ & 8.5 & 109 \\
\hline 13 & 0522 & 3.1 & 1,000 & 496 & 8.0 & 27.6 & - & 8.5 & 110 \\
\hline 13 & 0523 & 5.3 & 1,000 & 496 & 8.0 & 27.6 & $\cdots$ & 8.4 & 109 \\
\hline 13 & 0523 & 10 & 1,000 & 496 & 8.0 & 27.6 & $\cdots$ & 8.3 & 108 \\
\hline 13 & 0524 & 15 & 1,000 & 496 & 8.0 & 27.6 & $\cdots$ & 8.3 & 107 \\
\hline 13 & 0524 & 20 & 1,000 & 496 & 8.0 & 27.6 & $\ldots$ & 8.3 & 107 \\
\hline 13 & 0525 & 23 & 1,000 & 496 & 8.0 & 27.5 & & 8.2 & 106 \\
\hline
\end{tabular}


Table 17. Water-quality data for station 391720081334701, Ohio River at river mile 183.0, June to October, 1995, Continued.

[ft = feet; $\mu \mathrm{S} / \mathrm{cm}=$ microsiemens per centimeter; ${ }^{\circ} \mathrm{C}=$ degrees Celsius; $\mathrm{mg} / \mathrm{L}=\mathrm{milligrams}$ per liter; - = data not collected]

\begin{tabular}{|c|c|c|c|c|c|c|c|c|c|}
\hline Date & Time & $\begin{array}{l}\text { Sampling } \\
\text { depth } \\
\text { (ft) }\end{array}$ & $\begin{array}{c}\text { Sample } \\
\text { location } \\
\text { (Et from } \\
\text { left bank) }\end{array}$ & $\begin{array}{l}\text { Specitic } \\
\text { conduct - } \\
\text { ance } \\
(\mu \mathrm{S} / \mathrm{cm})\end{array}$ & $\begin{array}{l}\mathrm{pH} \\
\text { (stan- } \\
\text { dard } \\
\text { units) }\end{array}$ & $\begin{array}{c}\text { Temper- } \\
\text { ature, } \\
\text { water } \\
\left({ }^{\circ} \mathrm{C}\right)\end{array}$ & $\begin{array}{l}\text { Trans - } \\
\text { parency } \\
\text { (Secchi } \\
\text { disk) } \\
\text { (ft) }\end{array}$ & $\begin{array}{c}\text { Dissolved } \\
\text { oxygen } \\
\text { (mg/L) }\end{array}$ & $\begin{array}{c}\text { Dissolved } \\
\text { oxygen } \\
\text { (percent } \\
\text { satura- } \\
\text { tion) }\end{array}$ \\
\hline
\end{tabular}

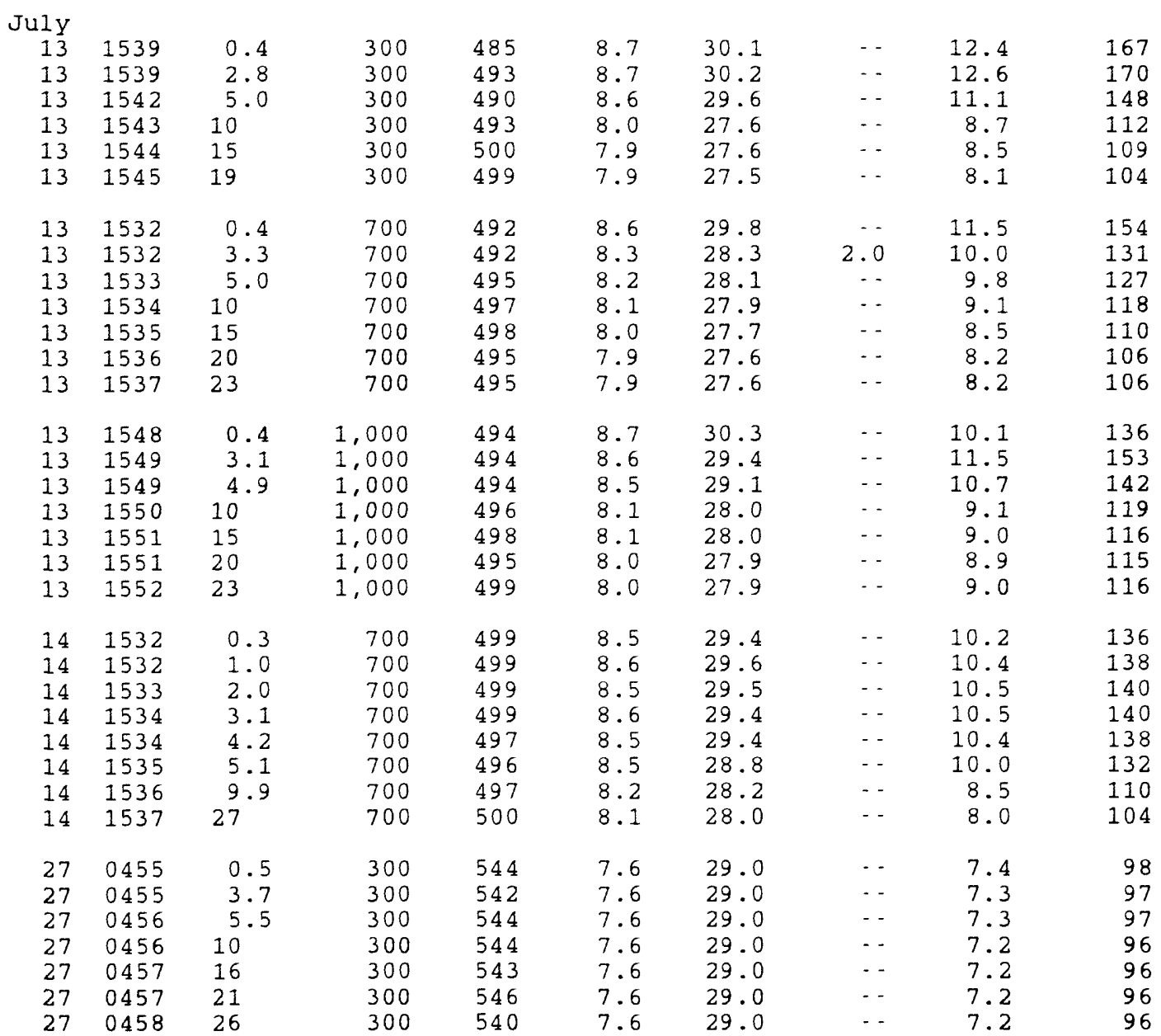


Table 17. Water-quality data for station 391720081334701, Ohio River at river mile 183.0, June to October, 1995, Continued.

[ft $=$ feet $; \mu \mathrm{s} / \mathrm{cm}=$ microsiemens per centumeter $;{ }^{\circ} \mathrm{C}=$ degrees celsius; $\mathrm{mg} / \mathrm{L}=\mathrm{mili}$ igrams per ilter; - = data not coliected]

\begin{tabular}{|c|c|c|c|c|c|c|c|c|c|}
\hline Date & Time & $\begin{array}{l}\text { Sampling } \\
\text { depth } \\
(f t)\end{array}$ & $\begin{array}{c}\text { Sample } \\
\text { location } \\
\text { (tt trom } \\
\text { lett bank) }\end{array}$ & $\begin{array}{l}\text { Specific } \\
\text { conduct- } \\
\text { ance } \\
(\mu \mathrm{S} / \mathrm{cm})\end{array}$ & $\begin{array}{l}\mathrm{pH} \\
\text { (stan- } \\
\text { dard } \\
\text { units) }\end{array}$ & $\begin{array}{c}\text { Temper- } \\
\text { ature, } \\
\text { water } \\
\left({ }^{\circ} \mathrm{C}\right)\end{array}$ & $\begin{array}{c}\text { Trans - } \\
\text { parency } \\
\text { (Secchi } \\
\text { disk) } \\
(\mathrm{ft})\end{array}$ & $\begin{array}{c}\text { Dissolved } \\
\text { oxygen } \\
(\mathrm{mg} / \mathrm{L})\end{array}$ & $\begin{array}{c}\text { Dissolved } \\
\text { oxygen } \\
\text { (percent } \\
\text { satura- } \\
\text { tron) }\end{array}$ \\
\hline \multicolumn{10}{|l|}{ July } \\
\hline 27 & 0450 & 0.5 & 700 & 550 & 7.6 & 29.1 & $\cdots$ & 7.4 & 99 \\
\hline 27 & 0450 & 3.6 & 700 & 548 & 7.6 & 29.1 & $\cdots$ & 7.4 & 98 \\
\hline 27 & 0451 & 5.6 & 700 & 548 & 7.6 & 29.1 & $\cdots$ & 7.3 & 97 \\
\hline 27 & 0451 & 11 & 700 & 548 & 7.6 & 29.1 & $\cdots$ & 7.3 & 97 \\
\hline 27 & 0452 & 16 & 700 & 547 & 7.6 & 29.1 & $\cdots$ & 7.3 & 97 \\
\hline 27 & 0452 & 21 & 700 & 547 & 7.6 & 29.1 & $\cdots$ & 7.3 & 97 \\
\hline 27 & 0453 & 25 & 700 & 548 & 7.6 & 29.1 & $\cdots$ & 7.2 & 96 \\
\hline 27 & 0453 & 26 & 700 & 548 & 7.6 & 29.1 & $\cdots$ & 7.3 & 97 \\
\hline 27 & 0446 & 0.5 & 1,000 & 562 & 7.7 & 29.1 & $\cdots$ & 7.5 & 99 \\
\hline 27 & 0446 & 3.6 & 1,000 & 558 & 7.6 & 29.1 & $\cdots$ & 7.4 & 98 \\
\hline 27 & 0447 & 5.9 & 1,000 & 559 & 7.6 & 29.2 & - & 7.4 & 98 \\
\hline 27 & 0447 & 10 & 1,000 & 558 & 7.6 & 29.2 & $\cdots$ & 7.4 & 98 \\
\hline 27 & 0448 & 16 & 1,000 & 563 & 7.6 & 29.2 & $\cdots$ & 7.3 & 98 \\
\hline 27 & 0448 & 20 & 1,000 & 561 & 7.6 & 29.1 & - & 7.3 & 97 \\
\hline 27 & 1334 & 0.6 & 300 & 531 & 7.8 & 29.9 & $\cdots$ & 8.4 & 112 \\
\hline 27 & 1334 & 3.1 & 300 & 530 & 7.7 & 29.3 & $\cdots$ & 7.6 & 101 \\
\hline 27 & 1335 & 5.1 & 300 & 528 & 7.6 & 29.2 & $-\cdot$ & 7.3 & 97 \\
\hline 27 & 1335 & 10 & 300 & 527 & 7.5 & 29.0 & $\cdots$ & 6.9 & 91 \\
\hline 27 & 1336 & 15 & 300 & 527 & 7.5 & 29.0 & $\cdots$ & 6.8 & 90 \\
\hline 27 & 1336 & 20 & 300 & 526 & 7.5 & 29.0 & $\cdots$ & 6.8 & 90 \\
\hline 27 & 1337 & 25 & 300 & 527 & 7.5 & 29.0 & $\cdots$ & 6.8 & 89 \\
\hline 27 & 1345 & 0.2 & 700 & 535 & 7.7 & 29.8 & -- & 7.7 & 103 \\
\hline 27 & 1345 & 3.0 & 700 & 535 & 7.7 & 30.0 & - & 7.8 & 104 \\
\hline 27 & 1346 & 5.1 & 700 & 537 & 7.7 & 29.6 & 3.5 & 7.5 & 100 \\
\hline 27 & 1347 & 10 & 700 & 538 & 7.6 & 29.4 & - & 7.2 & 96 \\
\hline 27 & 1347 & 15 & 700 & 539 & 7.6 & 29.2 & $\cdots$ & 6.8 & 91 \\
\hline 27 & 1348 & 20 & 700 & 543 & 7.5 & 29.1 & $\cdots$ & 6.6 & 88 \\
\hline 27 & 1348 & 25 & 700 & 547 & 7.5 & 29.1 & - & 6.6 & 87 \\
\hline 27 & 1349 & 27 & 700 & 545 & 7.5 & 29.1 & $\cdots$ & 6.6 & 88 \\
\hline
\end{tabular}


Table 17. Water-quality data for station 391720081334701, Ohio River at river mile 183.0, June to october, 1995, Continued.

[ft $=$ feet $; \mu \mathrm{S} / \mathrm{cm}=$ microsiemens per centimeter $;{ }^{\circ} \mathrm{C}=$ degrees Celsius: $\mathrm{mg} / \mathrm{L}=\mathrm{milligrams}$ per liter;.. = data not collected]

\begin{tabular}{|c|c|c|c|c|c|c|c|c|c|}
\hline Date & Time & $\begin{array}{l}\text { Sampling } \\
\text { depth } \\
\text { (ft) }\end{array}$ & $\begin{array}{c}\text { Sample } \\
\text { location } \\
\text { (ft from } \\
\text { left bank) }\end{array}$ & $\begin{array}{l}\text { Specific } \\
\text { conduct- } \\
\text { ance } \\
(\mu \mathrm{s} / \mathrm{cm})\end{array}$ & $\begin{array}{l}\mathrm{pH} \\
\text { (stan. } \\
\text { dard } \\
\text { units) }\end{array}$ & $\begin{array}{c}\text { Temper- } \\
\text { ature, } \\
\text { water } \\
\left({ }^{\circ} \mathrm{C}\right)\end{array}$ & $\begin{array}{l}\text { Trans- } \\
\text { parency } \\
\text { (secchl } \\
\text { disk) } \\
\text { (It) }\end{array}$ & $\begin{array}{l}\text { Dissolved } \\
\text { oxygen } \\
(\mathrm{mg} / \mathrm{L})\end{array}$ & $\begin{array}{c}\text { Dissolved } \\
\text { oxygen } \\
\text { (percent } \\
\text { satura- } \\
\text { tion) }\end{array}$ \\
\hline
\end{tabular}

$\begin{array}{rccc}\text { July } & & & \\ 27 & 1339 & 0.6 & 1,000 \\ 27 & 1339 & 3.1 & 1,000 \\ 27 & 1340 & 5.0 & 1,000 \\ 27 & 1340 & 10 & 1,000 \\ 27 & 1341 & 15 & 1,000 \\ 27 & 1341 & 20 & 1,000 \\ 27 & 1342 & 24 & 1,000\end{array}$

551
554
554
554
552
553
553

$\begin{array}{ll}7.9 & 30.5 \\ 7.9 & 29.8 \\ 7.8 & 29.5 \\ 7.6 & 29.2 \\ 7.6 & 29.2 \\ 7.6 & 29.2 \\ 7.6 & 29.2\end{array}$

$\begin{array}{llr}\ldots & 8.4 & 113 \\ \ldots & 8.3 & 111 \\ \ldots & 7.6 & 101 \\ \ldots & 7.0 & 92 \\ \ldots & 6.8 & 90 \\ \ldots & 6.8 & 90 \\ \ldots & 6.7 & 89\end{array}$

August

$\begin{array}{llllllllll}10 & 1513 & 0.9 & 300 & 419 & 7.6 & 27.6 & \ldots & 7.0 & 90 \\ 10 & 1513 & 3.5 & 300 & 415 & 7.6 & 26.2 & \ldots & 6.7 & 84 \\ 10 & 1514 & 5.5 & 300 & 415 & 7.5 & 26.1 & \ldots & 6.6 & 84 \\ 10 & 1514 & 10 & 300 & 414 & 7.5 & 26.0 & \ldots & 6.6 & 83 \\ 10 & 1515 & 15 & 300 & 416 & 7.5 & 26.0 & \ldots & 6.6 & 83 \\ 10 & 1515 & 20 & 300 & 411 & 7.5 & 25.9 & \ldots & 6.5 & 82 \\ 10 & 1516 & 23 & 300 & 410 & 7.5 & 25.9 & \ldots & 6.4 & 81 \\ 10 & 1518 & 1.1 & 700 & 420 & 7.6 & 27.5 & \ldots & 7.0 & 90 \\ 10 & 1518 & 3.4 & 700 & 417 & 7.6 & 26.4 & \ldots & 6.8 & 86 \\ 10 & 1519 & 5.5 & 700 & 415 & 7.6 & 26.2 & \ldots & 6.7 & 84 \\ 10 & 1519 & 10 & 700 & 417 & 7.6 & 26.1 & \ldots & 6.7 & 84 \\ 10 & 1520 & 15 & 700 & 415 & 7.5 & 26.1 & \ldots & 6.7 & 84 \\ 10 & 1520 & 20 & 700 & 420 & 7.6 & 26.0 & \ldots & 6.7 & 85 \\ 10 & 1521 & 25 & 700 & 420 & 7.6 & 26.1 & \ldots & 6.7 & 84 \\ 10 & 1521 & 28 & 700 & 417 & 7.6 & 26.1 & \ldots & 6.7 & 84 \\ 10 & 1523 & 1.0 & 1,000 & 424 & 7.6 & 26.7 & \ldots & 6.8 & 86 \\ 10 & 1523 & 3.4 & 1,000 & 419 & 7.6 & 26.3 & \ldots & 6.8 & 86 \\ 10 & 1524 & 5.5 & 1,000 & 419 & 7.6 & 26.1 & \ldots & 6.8 & 85 \\ 10 & 1524 & 10 & 1,000 & 419 & 7.6 & 26.1 & \ldots & 6.8 & 85 \\ 10 & 1525 & 15 & 1,000 & 416 & 7.6 & 26.1 & \ldots & 6.8 & 85 \\ 10 & 1525 & 20 & 1,000 & 417 & 7.6 & 26.1 & \ldots & 6.7 & 85 \\ 10 & 1526 & 25 & 1,000 & 418 & 7.6 & 26.1 & \ldots & 6.7 & 84\end{array}$


Table 17. Water-quality data for station 391720081334701, Ohio River at river mile 183.0, June to October, 1995, Continued.

[Et $=$ Eeet $; \mu \mathrm{S} / \mathrm{cm}=$ microslemens per centimeter; ${ }^{\circ} \mathrm{C}=$ degrees Celsius; $\mathrm{mg} / \mathrm{L}=\mathrm{mili}$ igrams per liter; $\cdots$ = data not collected]

\begin{tabular}{|c|c|c|c|c|c|c|c|c|c|}
\hline Date & Time & $\begin{array}{l}\text { Sampling } \\
\text { depth } \\
\text { (ft) }\end{array}$ & $\begin{array}{c}\text { Sample } \\
\text { location } \\
\text { (ft trom } \\
\text { left bank) }\end{array}$ & $\begin{array}{l}\text { Specific } \\
\text { conduct- } \\
\text { ance } \\
(\mu \mathrm{s} / \mathrm{cm})\end{array}$ & $\begin{array}{l}\mathrm{pH} \\
\text { (stan- } \\
\text { dard } \\
\text { units) }\end{array}$ & $\begin{array}{c}\text { Temper- } \\
\text { ature, } \\
\text { water } \\
\left({ }^{\circ} \mathrm{C}\right)\end{array}$ & $\begin{array}{l}\text { Trans - } \\
\text { parency } \\
\text { (secchl } \\
\text { disk) } \\
\text { (Et) }\end{array}$ & $\begin{array}{l}\text { Dissolved } \\
\text { oxygen } \\
(\mathrm{mg} / \mathrm{L})\end{array}$ & $\begin{array}{c}\text { Dissolved } \\
\text { oxygen } \\
\text { (percent } \\
\text { satura- } \\
\text { tlon) }\end{array}$ \\
\hline
\end{tabular}

\begin{tabular}{|c|c|c|c|c|c|c|c|c|c|}
\hline Augu & & & & & & & & & \\
\hline 24 & 0559 & 0.5 & 300 & 546 & 7.8 & 29.2 & $\cdots$ & 7.3 & 97 \\
\hline 24 & 0559 & 2.9 & 300 & 552 & 7.9 & 29.2 & $\cdots$ & 7.3 & 97 \\
\hline 24 & 0600 & 5.2 & 300 & 550 & 7.8 & 29.2 & $\cdots$ & 7.2 & 96 \\
\hline 24 & 0600 & 10 & 300 & 552 & 7.8 & 29.2 & $\cdots$ & 7.2 & 95 \\
\hline 24 & 0601 & 15 & 300 & 554 & 7.8 & 29.2 & $\cdots$ & 7.2 & 95 \\
\hline 24 & 0601 & 20 & 300 & 552 & 7.8 & 29.2 & - & 7.2 & 96 \\
\hline 24 & 0602 & 25 & 300 & 553 & 7.8 & 29.2 & - & 7.2 & 95 \\
\hline 24 & 0604 & 0.4 & 700 & 551 & 7.8 & 29.2 & $\cdots$ & 7.3 & 97 \\
\hline 24 & 0604 & 3.3 & 700 & 541 & 7.8 & 29.2 & $\cdots$ & 7.3 & 96 \\
\hline 24 & 0605 & 5.8 & 700 & 545 & 7.8 & 29.2 & - & 7.3 & 96 \\
\hline 24 & 0605 & 9.8 & 700 & 546 & 7.8 & 29.2 & $\cdots$ & 7.3 & 96 \\
\hline 24 & 0606 & 15 & 700 & 547 & 7.8 & 29.2 & $\cdots$ & 7.2 & 96 \\
\hline 24 & 0606 & 20 & 700 & 543 & 7.8 & 29.2 & $\cdots$ & 7.2 & 96 \\
\hline 24 & 0607 & 25 & 700 & 552 & 7.8 & 29.2 & $\cdots$ & 7.2 & 96 \\
\hline 24 & 0607 & 27 & 700 & 541 & 7.8 & 29.2 & $\cdots$ & 7.2 & 95 \\
\hline 24 & 0609 & 0.8 & 1,000 & 542 & 7.8 & 29.2 & $\cdots$ & 7.2 & 95 \\
\hline 24 & 0609 & 3.3 & 1,000 & 541 & 7.8 & 29.2 & $\cdots$ & 7.2 & 95 \\
\hline 24 & 0610 & 5.4 & 1,000 & 542 & 7.8 & 29.2 & $\cdots$ & 7.2 & 95 \\
\hline 24 & 0610 & 9.8 & 1,000 & 542 & 7.8 & 29.2 & - & 7.2 & 95 \\
\hline 24 & 0611 & 15 & 1,000 & 544 & 7.8 & 29.2 & $\cdots$ & 7.1 & 95 \\
\hline 24 & 0611 & 20 & 1,000 & 543 & 7.8 & 29.2 & $\cdots$ & 7.1 & 94 \\
\hline 24 & 0612 & 23 & 1,000 & 549 & 7.8 & 29.2 & $\cdots$ & 6.9 & 92 \\
\hline 24 & 1248 & 0.8 & 300 & 554 & 8.4 & 30.8 & $\cdots$ & 10.3 & 140 \\
\hline 24 & 1248 & 3.3 & 300 & 555 & 8.3 & 29.6 & $\cdots$ & 9.3 & 124 \\
\hline 24 & 1249 & 5.1 & 300 & 553 & 8.2 & 29.3 & $\cdots$ & 9.0 & 119 \\
\hline 24 & 1249 & 10 & 300 & 554 & 8.0 & 29.0 & - & 7.6 & 101 \\
\hline 24 & 1250 & 15 & 300 & 556 & 7.9 & 29.0 & - & 7.4 & 97 \\
\hline 24 & 1250 & 20 & 300 & 550 & 7.9 & 28.9 & $\cdots$ & 7.2 & 95 \\
\hline 24 & 1251 & 25 & 300 & 549 & 7.9 & 28.9 & $\cdots$ & 7.3 & 96 \\
\hline 24 & 1251 & 26 & 300 & 549 & 7.9 & 28.9 & $\cdots$ & 7.3 & 96 \\
\hline
\end{tabular}


Table 17. Water-quality data for station 391720081334701, Ohio River at river mile 183.0, June to October, 1995, Continued.

[ft $=$ feet; $\mu \mathrm{S} / \mathrm{cm}=$ microsiemens per centimeter; ${ }^{\circ} \mathrm{C}=$ degrees celsius;

$\mathrm{mg} / \mathrm{L}=\mathrm{mili}$ igrams per liter; $-\cdot$ = data not collected]

\begin{tabular}{|c|c|c|c|c|c|c|c|c|c|}
\hline Date & Time & $\begin{array}{l}\text { Sampling } \\
\text { depth } \\
\text { (Et) }\end{array}$ & $\begin{array}{c}\text { Sample } \\
\text { location } \\
\text { (ft from } \\
\text { left bank) }\end{array}$ & $\begin{array}{l}\text { Specific } \\
\text { conduct - } \\
\text { ance } \\
(\mu \mathrm{s} / \mathrm{cm})\end{array}$ & $\begin{array}{l}\mathrm{pH} \\
\text { (stan- } \\
\text { dard } \\
\text { units) }\end{array}$ & $\begin{array}{c}\text { Temper- } \\
\text { ature. } \\
\text { water } \\
\left({ }^{\circ} \mathrm{C}\right)\end{array}$ & $\begin{array}{l}\text { Trans- } \\
\text { parency } \\
\text { (Secchi } \\
\text { disk) } \\
\text { (Et) }\end{array}$ & $\begin{array}{c}\text { Dissolved } \\
\text { oxygen } \\
\text { (mg/L) }\end{array}$ & $\begin{array}{c}\text { Dissolved } \\
\text { oxygen } \\
\text { (percent } \\
\text { satura- } \\
\text { tion) }\end{array}$ \\
\hline
\end{tabular}

August

\begin{tabular}{|c|c|c|c|c|c|c|c|c|c|}
\hline \\
\hline 24 & 1242 & 1.1 & 700 & 554 & 8.1 & 29.6 & $\cdots$ & 8.3 & 111 \\
\hline 24 & 1242 & 3.4 & 700 & 553 & 8.0 & 29.1 & 2.5 & 7.6 & 100 \\
\hline 24 & 1243 & 5.4 & 700 & 555 & 7.9 & 29.1 & - - & 7.5 & 99 \\
\hline 24 & 1243 & 10 & 700 & 554 & 7.9 & 29.1 & -- & 7.3 & 97 \\
\hline 24 & 1244 & 15 & 700 & 553 & 7.9 & 29.0 & $\cdots$ & 7.3 & 96 \\
\hline 24 & 1244 & 20 & 700 & 551 & 7.9 & 29.0 & - & 7.2 & 95 \\
\hline 24 & 1245 & 25 & 700 & 550 & 7.9 & 28.9 & $\cdots$ & 7.1 & 93 \\
\hline 24 & 1245 & 28 & 700 & 550 & 7.8 & 28.9 & $\cdots$ & 7.0 & 92 \\
\hline 24 & 1238 & 0.4 & 1,000 & 550 & 8.5 & 30.7 & $\cdots$ & 10.6 & 144 \\
\hline 24 & 1238 & 3.3 & 1,000 & 553 & 8.5 & 29.9 & $\cdots$ & 10.8 & 145 \\
\hline 24 & 1239 & 5.2 & 1,000 & 553 & 8.1 & $29 \cdot 3$ & $\cdots$ & 8.4 & 112 \\
\hline 24 & 1239 & 9.9 & 1,000 & 556 & 7.9 & 29.1 & $\cdots$ & 7.4 & 98 \\
\hline 24 & 1240 & 15 & 1,000 & 556 & 7.9 & 29.1 & $\cdots$ & 7.4 & 97 \\
\hline 24 & 1240 & 16 & 1,000 & 550 & 7.9 & 29.1 & $\cdots$ & 7.2 & 96 \\
\hline \multicolumn{10}{|c|}{ Sep tember } \\
\hline 06 & 0517 & 0.3 & 300 & 546 & 7.7 & 27.3 & $\cdots$ & 7.6 & 97 \\
\hline 06 & 0517 & 2.5 & 300 & 549 & 7.7 & 27.3 & $\cdots$ & 7.6 & 97 \\
\hline 06 & 0518 & 4.7 & 300 & 546 & 7.7 & 27.4 & $\cdots$ & $7 \cdot 6$ & 97 \\
\hline 06 & 0518 & 9.7 & 300 & 545 & 7.7 & 27.3 & -- & $7 \cdot 2$ & 93 \\
\hline 06 & 0519 & 15 & 300 & 549 & 7.7 & 27.4 & $\cdots$ & 7.2 & 93 \\
\hline 06 & 0519 & 20 & 300 & 547 & 7.7 & 27.3 & $\cdots$ & 7.2 & 92 \\
\hline 06 & 0520 & 25 & 300 & 543 & 7.7 & $27 \cdot 3$ & $\cdots$ & 7.1 & 90 \\
\hline 06 & 0520 & 27 & 300 & 544 & 7.7 & 27.3 & $\cdots$ & 7.0 & 89 \\
\hline 06 & 0512 & 0.4 & 700 & 546 & 7.7 & 27.4 & $\cdots$ & 7.6 & 97 \\
\hline 06 & 0515 & 3.0 & 700 & 547 & 7.7 & 27.4 & $\cdots$ & 7.5 & 96 \\
\hline 06 & 0512 & 5.0 & 700 & 550 & 7.7 & 27.4 & $\cdots$ & 7.5 & 97 \\
\hline 06 & 0513 & 9.5 & 700 & 551 & 7.7 & 27.4 & $\cdots$ & 7.5 & 96 \\
\hline 06 & 0513 & 15 & 700 & 547 & 7.7 & 27.4 & $\cdots$ & 7.5 & 96 \\
\hline 06 & 0514 & 20 & 700 & 549 & 7.7 & 27.4 & $\cdots$ & 7.5 & 95 \\
\hline 06 & 0515 & 22 & 700 & 545 & 7.7 & 27.4 & $\cdots$ & 7.2 & 92 \\
\hline 06 & 0514 & 24 & 700 & 551 & 7.7 & 27.4 & -- & 7.4 & 95 \\
\hline
\end{tabular}


Table 17. Water-quality data for station 391720081334701, Ohio River at river mile 183.0, June to October, 1995, Continued.

\begin{tabular}{|c|c|c|c|c|c|c|c|c|c|}
\hline Date & Time & $\begin{array}{l}\text { Sampling } \\
\text { depth } \\
\text { (ft) }\end{array}$ & $\begin{array}{c}\text { Sample } \\
\text { location } \\
\text { (ft Erom } \\
\text { left bank) }\end{array}$ & $\begin{array}{l}\text { Specific } \\
\text { conduct- } \\
\text { ance } \\
(\mu \mathrm{S} / \mathrm{cm})\end{array}$ & $\begin{array}{l}\text { pH } \\
\text { (stan- } \\
\text { dard } \\
\text { units) }\end{array}$ & $\begin{array}{c}\text { Temper- } \\
\text { ature. } \\
\text { water } \\
\left({ }^{\circ} \mathrm{C}\right)\end{array}$ & $\begin{array}{l}\text { Trans } \\
\text { parency } \\
\text { (SecchI } \\
\text { disk) } \\
\text { (ft) }\end{array}$ & $\begin{array}{c}\text { Dissolved } \\
\text { oxygen } \\
(\mathrm{mg} / \mathrm{L})\end{array}$ & $\begin{array}{r}\text { Dissoly } \\
\text { oxyge } \\
\text { (perce } \\
\text { satur } \\
\text { tion. }\end{array}$ \\
\hline \multicolumn{10}{|c|}{ September } \\
\hline 06 & 0507 & 0.4 & 1,000 & 547 & 7.7 & 27.3 & $\cdots$ & 7.5 & 95 \\
\hline 06 & 0507 & 3.1 & 1,000 & 547 & 7.7 & 27.4 & $\ldots$ & 7.5 & 96 \\
\hline 06 & 0508 & 4.7 & 1,000 & 549 & 7.7 & 27.4 & $\cdots$ & 7.5 & 96 \\
\hline 06 & 0508 & 9.6 & 1,000 & 549 & 7.7 & 27.4 & - & 7.5 & 96 \\
\hline 06 & 0509 & 15 & 1,000 & 548 & 7.7 & 27.4 & $\cdots$ & 7.5 & 96 \\
\hline 06 & 0509 & 20 & 1,000 & 549 & 7.7 & 27.4 & - & 7.5 & 96 \\
\hline 06 & 0510 & 21 & 1,000 & 546 & 7.7 & 27.4 & - & 7.3 & 93 \\
\hline 06 & 1335 & 0.8 & 300 & 548 & 8.1 & 28.4 & $\cdots$ & 9.8 & 127 \\
\hline 06 & 1335 & 2.9 & 300 & 549 & 8.2 & 28.3 & -- & 9.9 & 129 \\
\hline 06 & 1336 & 5.2 & 300 & 549 & 8.1 & 28.0 & $\cdots$ & 9.7 & 125 \\
\hline 06 & 1336 & 9.4 & 300 & 548 & 7.8 & 27.6 & $\cdots$ & 8.4 & 107 \\
\hline 06 & 1337 & 15 & 300 & 548 & 7.6 & 27.2 & -- & 7.7 & 98 \\
\hline 06 & 1337 & 18 & 300 & 548 & 7.6 & 27.2 & $\cdots$ & 7.5 & 95 \\
\hline 06 & 1352 & 0.7 & 700 & 548 & 8.2 & 28.6 & $\cdots$ & 9.8 & 128 \\
\hline 06 & 1352 & 2.9 & 700 & 548 & 7.9 & 28.1 & - & 9.0 & 116 \\
\hline 06 & 1353 & 5.1 & 700 & 550 & 7.7 & 27.5 & $\cdots$ & 7.8 & 99 \\
\hline 06 & 1353 & 9.6 & 700 & 550 & 7.6 & 27.5 & - & 7.6 & 97 \\
\hline 06 & 1354 & 15 & 700 & 551 & 7.6 & 27.4 & $\cdots$ & 7.5 & 96 \\
\hline 06 & 1354 & 19 & 700 & 550 & 7.6 & 27.4 & - & 7.5 & 96 \\
\hline 06 & 1355 & 24 & 700 & 542 & 7.6 & 27.3 & - & 7.5 & 96 \\
\hline 06 & 1355 & 27 & 700 & 544 & 7.6 & 27.3 & $\cdots$ & 7.5 & 95 \\
\hline 06 & 1357 & 0.6 & 1,000 & 547 & 8.1 & 28.3 & $\cdots$ & 9.3 & 121 \\
\hline 06 & 1357 & 2.9 & 1,000 & 551 & 7.8 & 27.8 & - & 8.1 & 105 \\
\hline 06 & 1358 & 4.9 & 1,000 & 550 & 7.7 & 27.6 & -- & 7.9 & 101 \\
\hline 06 & 1358 & 9.7 & 1,000 & 548 & 7.6 & 27.5 & $\cdots$ & 7.5 & 96 \\
\hline 06 & 1359 & 15 & 1,000 & 551 & 7.6 & 27.4 & $\cdots$ & 7.5 & 96 \\
\hline 06 & 1359 & 20 & 1,000 & 551 & 7.6 & 27.3 & $\cdots$ & 7.4 & 95 \\
\hline 06 & 1400 & 24 & 1,000 & 551 & 7.6 & 27.2 & - & 7.3 & 93 \\
\hline 20 & 0520 & 0.4 & 300 & 681 & 7.5 & 24.0 & -- & 6.5 & 79 \\
\hline 20 & 0520 & 2.9 & 300 & 681 & 7.5 & 24.0 & $\cdots$ & 6.4 & 77 \\
\hline 20 & 0521 & 5.2 & 300 & 680 & 7.5 & 24.0 & - & 6.3 & 76 \\
\hline 20 & 0521 & 9.8 & 300 & 680 & 7.5 & 24.0 & - - & 6.3 & 77 \\
\hline 20 & 0522 & 15 & 300 & 681 & 7.5 & 24.0 & $\cdots$ & 6.3 & 76 \\
\hline 20 & 0522 & 20 & 300 & 682 & 7.5 & 24.0 & - & 6.3 & 76 \\
\hline 20 & 0523 & 25 & 300 & 680 & 7.5 & 24.0 & $\cdots$ & 6.3 & 76 \\
\hline 20 & 0523 & 27 & 300 & 680 & 7.5 & 24.0 & - & 6.1 & 74 \\
\hline
\end{tabular}


Table 17. Water-quality data for station 391720081334701, Ohio River at river mile 183.0, June to October, 1995, Continued.

[et $=$ feet $; \mu \mathrm{S} / \mathrm{cm}=$ microsiemens per centımeter $;{ }^{\circ} \mathrm{C}=$ degrees Celsius: $\mathrm{mg} / \mathrm{L}=\mathrm{mll}$ ligrams per liter; . = data not collected]

\begin{tabular}{|c|c|c|c|c|c|c|c|c|c|}
\hline Date & Time & $\begin{array}{l}\text { Sampling } \\
\text { depth } \\
\text { (ft) }\end{array}$ & $\begin{array}{l}\text { Sample } \\
\text { location } \\
\text { (ft from } \\
\text { left bank) }\end{array}$ & $\begin{array}{l}\text { Speciflc } \\
\text { conduct- } \\
\text { ance } \\
(\mu \mathrm{S} / \mathrm{cm})\end{array}$ & $\begin{array}{l}\text { pH } \\
\text { (stan- } \\
\text { dard } \\
\text { units) }\end{array}$ & $\begin{array}{c}\text { Temper- } \\
\text { ature, } \\
\text { water } \\
\left({ }^{\circ} \mathrm{C}\right)\end{array}$ & $\begin{array}{c}\text { Trans- } \\
\text { parency } \\
\text { (secchi } \\
\text { disk) } \\
(t t)\end{array}$ & $\begin{array}{c}\text { Dissolved } \\
\text { oxygen } \\
\text { (mg/L) }\end{array}$ & $\begin{array}{c}\text { Dissolved } \\
\text { oxygen } \\
\text { (percent } \\
\text { satura- } \\
\text { tion) }\end{array}$ \\
\hline
\end{tabular}

$\begin{array}{lcccc}\text { September } & & & \\ 20 & 0525 & 0.3 & 700 & 678 \\ 20 & 0525 & 3.3 & 700 & 684 \\ 20 & 0526 & 5.2 & 700 & 679 \\ 20 & 0526 & 9.8 & 700 & 678 \\ 20 & 0527 & 15 & 700 & 680 \\ 20 & 0527 & 20 & 700 & 678 \\ 20 & 0528 & 25 & 700 & 682 \\ 20 & 0528 & 27 & 700 & 684 \\ & & & & \\ 20 & 0530 & 0.5 & 1,000 & 681 \\ 20 & 0530 & 3.2 & 1,000 & 683 \\ 20 & 0531 & 5.4 & 1,000 & 681 \\ 20 & 0531 & 9.8 & 1,000 & 679 \\ 20 & 0532 & 15 & 1,000 & 681 \\ 20 & 0532 & 20 & 1,000 & 680 \\ 20 & 0533 & 24 & 1,000 & 681 \\ & & & & \\ 20 & 1217 & 0.6 & 300 & 684 \\ 20 & 1218 & 3.0 & 300 & 686 \\ 20 & 1218 & 5.1 & 300 & 685 \\ 20 & 1219 & 9.9 & 300 & 686 \\ 20 & 1219 & 14 & 300 & 686 \\ 20 & 1220 & 19 & 300 & 685 \\ & & & & \\ 20 & 1223 & 1.2 & 700 & 687 \\ 20 & 1223 & 3.2 & 700 & 686 \\ 20 & 1224 & 5.7 & 700 & 687 \\ 20 & 1224 & 10 & 700 & 686 \\ 20 & 1225 & 15 & 700 & 686 \\ 20 & 1225 & 20 & 700 & 687 \\ 20 & 1226 & 25 & 700 & 685 \\ 20 & 1226 & 26 & 700 & 686\end{array}$

7.5
7.5
7.5
7.5
7.5
7.5
7.5
7.5
7.5
7.5
7.5
7.5
7.5
7.5
7.5
7.6
7.5
7.5
7.5
7.5
7.5
7.6
7.5
7.5
7.5
7.5
7.5
7.5
7.5

23.9

24.0

24.0

23.9

24.0

24.0

24.0

24.0

23.9

23.9

23.9

23.9

23.9

23.9

23.9

23.7

23.8

23.8

23.8

23.8

23.8

23.8

23.8

23.8

23.8

23.8

23.8

23.8

23.8
-

6.5

6.5

․ 6.4

- 6.4

- 6.4

. 6.4

- 6.3

- 6.2

$\cdots$
.

5.8
6.2

.. 6.2

. 6.1

. 6.1

- 6.0

- 6.1

$\begin{array}{ll}- & 6.3 \\ \ldots & 6.2\end{array}$

. 6.2

. 6.2

. 6.1

6.1
5.9

6.4

6.4

6.3

6.4

6.3

6.3

6.3

-. 6.2

$\begin{array}{ll}5 & 79 \\ 5 & 79 \\ 4 & 78 \\ 4 & 77 \\ 4 & 78 \\ 4 & 77 \\ 3 & 76 \\ 2 & 75 \\ & \\ 8 & 71 \\ 2 & 75 \\ 2 & 75 \\ 1 & 74 \\ 1 & 74 \\ 0 & 73 \\ 1 & 74 \\ .3 & 75 \\ 2 & 75 \\ 2 & 74 \\ 2 & 74 \\ 1 & 74 \\ 9 & 71 \\ & \\ 4 & 77 \\ 4 & 77 \\ 3 & 76 \\ 4 & 77 \\ .3 & 76 \\ 3 & 76 \\ .3 & 76 \\ 2 & 75\end{array}$


Table 17. Water-quality data for station 391720081334701, Ohio River at river mile 183.0, June to October, 1995, Continued.

[Et $=$ feet $; \mu \mathrm{S} / \mathrm{cm}=$ microsiemens per centimeter; ${ }^{\circ} \mathrm{C}=$ degrees Celsius;

$\mathrm{mg} / \mathrm{L}=\mathrm{milligrams}$ per liter; $\cdot \cdot$ = data not collected]

\begin{tabular}{|c|c|c|c|c|c|c|c|c|c|}
\hline Date & Time & $\begin{array}{l}\text { Sampling } \\
\text { depth } \\
\text { (ft) }\end{array}$ & $\begin{array}{l}\text { Sample } \\
\text { location } \\
\text { (ft from } \\
\text { left bank) }\end{array}$ & $\begin{array}{l}\text { Specific } \\
\text { conduct- } \\
\text { ance } \\
(\mu \mathrm{S} / \mathrm{cm})\end{array}$ & $\begin{array}{l}\mathrm{pH} \\
\text { (stan- } \\
\text { dard } \\
\text { units) }\end{array}$ & $\begin{array}{c}\text { Temper- } \\
\text { ature, } \\
\text { water } \\
\left({ }^{\circ} \mathrm{C}\right)\end{array}$ & $\begin{array}{l}\text { Trans- } \\
\text { parency } \\
\text { (Secchi } \\
\text { disk) } \\
\text { (ft) }\end{array}$ & $\begin{array}{l}\text { Dissolved } \\
\text { oxygen } \\
(\mathrm{mg} / \mathrm{L})\end{array}$ & $\begin{array}{c}\text { Dissolved } \\
\text { oxygen } \\
\text { (percent } \\
\text { satura- } \\
\text { tron) }\end{array}$ \\
\hline
\end{tabular}

September

$\begin{array}{llllllllll}20 & 1228 & 0.5 & 1,000 & 673 & 7.5 & 23.8 & \ldots & 6.5 & 78 \\ 20 & 1229 & 3.0 & 1,000 & 686 & 7.5 & 23.8 & \ldots & 6.4 & 77 \\ 20 & 1229 & 5.0 & 1,000 & 687 & 7.5 & 23.8 & \ldots & 6.4 & 77 \\ 20 & 1230 & 10 & 1,000 & 687 & 7.5 & 23.9 & \ldots & 6.5 & 78 \\ 20 & 1231 & 15 & 1,000 & 686 & 7.5 & 23.9 & \ldots & 6.5 & 78 \\ 20 & 1231 & 20 & 1,000 & 686 & 7.5 & 23.9 & \ldots & 6.4 & 77 \\ 20 & 1232 & 23 & 1,000 & 687 & 7.5 & 23.9 & \ldots & 6.3 & 76\end{array}$

October

$\begin{array}{llllllllll}05 & 0816 & 0.5 & 300 & 621 & 7.3 & 21.7 & \ldots & 6.7 & 77 \\ 05 & 0816 & 3.1 & 300 & 627 & 7.3 & 21.7 & \ldots & 6.7 & 77 \\ 05 & 0817 & 5.0 & 300 & 627 & 7.3 & 21.7 & \ldots & 6.7 & 77 \\ 05 & 0817 & 9.9 & 300 & 629 & 7.3 & 21.7 & \ldots & 6.7 & 77 \\ 05 & 0818 & 15 & 300 & 634 & 7.3 & 21.7 & \ldots & 6.7 & 77 \\ 05 & 0818 & 20 & 300 & 639 & 7.3 & 21.7 & \ldots & 6.7 & 77 \\ 05 & 0819 & 21 & 300 & 636 & 7.3 & 21.7 & \ldots & 6.6 & 76 \\ 05 & 0809 & 0.5 & 700 & 631 & 7.3 & 21.8 & \ldots & 6.8 & 79 \\ 05 & 0809 & 3.2 & 700 & 641 & 7.3 & 21.8 & \ldots & 6.8 & 79 \\ 05 & 0810 & 5.2 & 700 & 642 & 7.3 & 21.8 & \ldots & 6.7 & 78 \\ 05 & 0810 & 10 & 700 & 642 & 7.3 & 21.8 & \ldots & 6.7 & 78 \\ 05 & 0811 & 15 & 700 & 645 & 7.3 & 21.8 & \ldots & 6.7 & 78 \\ 05 & 0811 & 20 & 700 & 652 & 7.3 & 21.8 & \ldots & 6.7 & 77 \\ 05 & 0812 & 25 & 700 & 636 & 7.3 & 21.8 & \ldots & 6.7 & 77 \\ 05 & 0804 & 0.2 & 1,000 & 630 & 7.3 & 21.7 & \ldots & 7.0 & 81 \\ 05 & 0804 & 2.9 & 1,000 & 635 & 7.3 & 21.8 & \ldots & 6.8 & 78 \\ 05 & 0805 & 5.1 & 1,000 & 637 & 7.3 & 21.8 & \ldots & 6.8 & 78 \\ 05 & 0806 & 10 & 1,000 & 642 & 7.3 & 21.8 & \ldots & 6.7 & 78 \\ 05 & 0806 & 15 & 1,000 & 641 & 7.3 & 21.7 & \ldots & 6.7 & 78 \\ 05 & 0807 & 17 & 1,000 & 640 & 7.3 & 21.7 & \ldots & 6.7 & 78 \\ 05 & 1539 & 0.4 & 300 & 617 & 7.5 & 21.7 & \ldots & 6.7 & 78 \\ 05 & 1539 & 3.2 & 300 & 622 & 7.5 & 21.7 & \ldots & 6.6 & 77 \\ 05 & 1540 & 4.9 & 300 & 622 & 7.5 & 21.7 & \ldots & 6.6 & 77 \\ 05 & 1540 & 10 & 300 & 629 & 7.5 & 21.7 & \ldots & 6.7 & 77 \\ 05 & 1541 & 15 & 300 & 625 & 7.5 & 21.6 & \ldots & 6.6 & 76 \\ 05 & 1541 & 20 & 300 & 620 & 7.5 & 21.6 & \ldots & 6.5 & 75 \\ 05 & 1542 & 24 & 300 & 621 & 7.5 & 21.6 & \ldots & 6.5 & 75\end{array}$


Table 17. Water-quality data for station 391720081334701, Ohio River at river mile 183.0, June to october, 1995, continued.

[ft $=$ feet $; \mu \mathrm{s} / \mathrm{cm}=$ microsiemens per centimeter; ${ }^{\circ} \mathrm{C}=$ degrees Celsius; $\mathrm{mg} / \mathrm{L}=$ milligrams per liter; . - = data not collected]

\begin{tabular}{|c|c|c|c|c|c|c|c|c|c|}
\hline Date & Time & $\begin{array}{l}\text { Samp I ing } \\
\text { depth } \\
\text { (Et) }\end{array}$ & $\begin{array}{c}\text { Sample } \\
\text { location } \\
\text { (tt from } \\
\text { left bank) }\end{array}$ & $\begin{array}{l}\text { Specitic } \\
\text { conduct - } \\
\text { ance } \\
(\mu \mathrm{s} / \mathrm{cm})\end{array}$ & $\begin{array}{l}\text { pH } \\
\text { (stan- } \\
\text { dard } \\
\text { units) }\end{array}$ & $\begin{array}{c}\text { Temper- } \\
\text { ature. } \\
\text { water } \\
\left({ }^{\circ} \mathrm{C}\right)\end{array}$ & $\begin{array}{l}\text { Trans- } \\
\text { parency } \\
\text { (Secch } \\
\text { disk) } \\
\text { (Et) }\end{array}$ & $\begin{array}{l}\text { Dissolved } \\
\text { oxygen } \\
(\mathrm{mg} / \mathrm{L})\end{array}$ & $\begin{array}{c}\text { Dissolved } \\
\text { oxygen } \\
\text { (percent } \\
\text { satura- } \\
\text { tion) }\end{array}$ \\
\hline
\end{tabular}

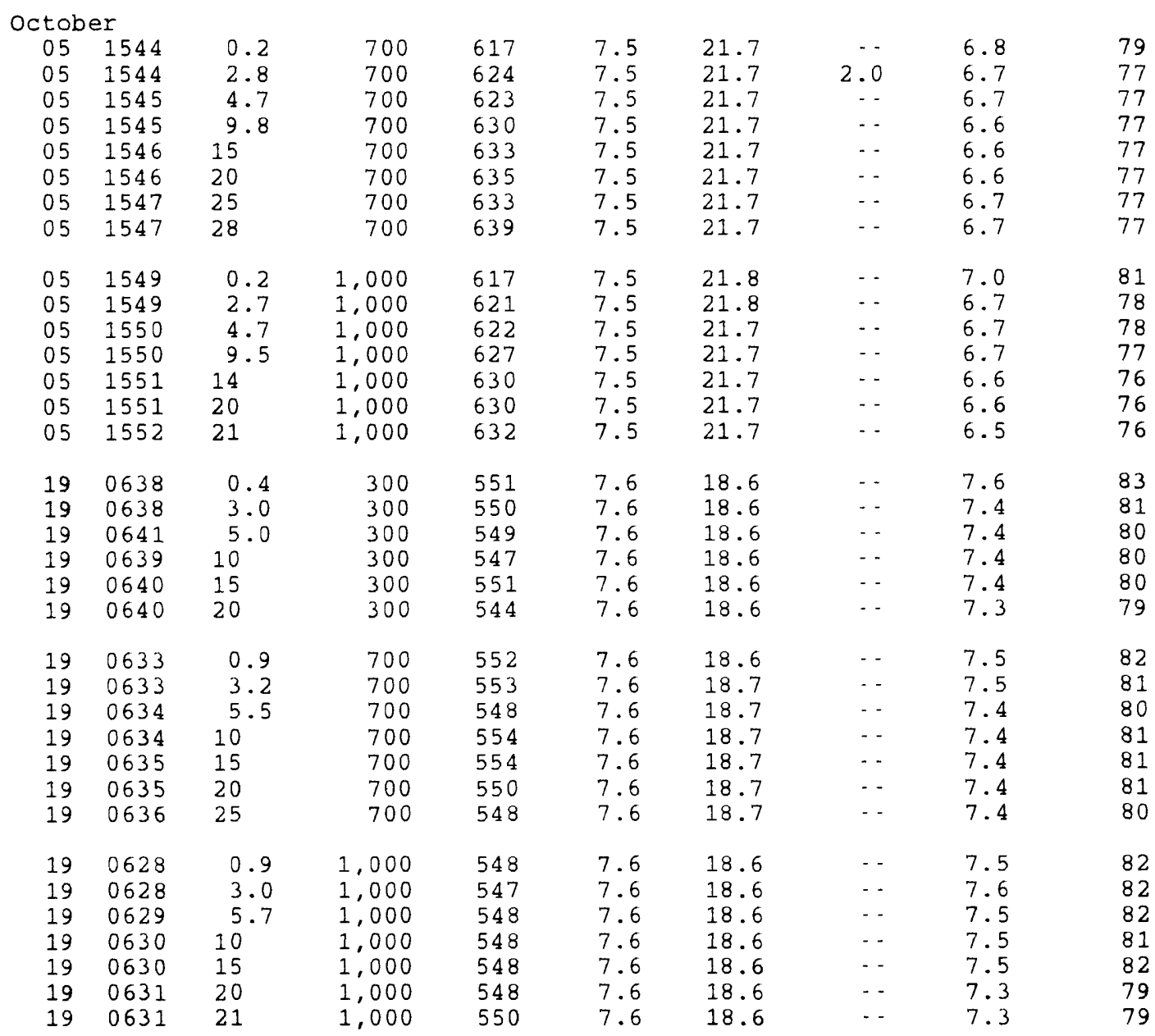


Table 17. Water-quality data for station 391720081334701, Ohio River at river mile 183.0, June to October, 1995, Continued.

[Et $=$ feet; $\mu \mathrm{s} / \mathrm{cm}=$ microsiemens per centimeter ${ }^{\circ} \mathrm{C}=$ degrees Celsius; $\mathrm{mg} / \mathrm{L}=\mathrm{mili}$ igrams per liter; - - = data not collected]

\begin{tabular}{|c|c|c|c|c|c|c|c|c|c|}
\hline Date & Time & $\begin{array}{l}\text { Sampling } \\
\text { depth } \\
( \pm t)\end{array}$ & $\begin{array}{c}\text { Sample } \\
\text { location } \\
\text { (tt from } \\
\text { left bank) }\end{array}$ & $\begin{array}{l}\text { Specific } \\
\text { conduct- } \\
\text { ance } \\
(\mu S / \mathrm{cm})\end{array}$ & $\begin{array}{l}\text { pH } \\
\text { (stan- } \\
\text { dard } \\
\text { units) }\end{array}$ & $\begin{array}{c}\text { Temper- } \\
\text { ature, } \\
\text { water } \\
\left({ }^{\circ} \mathrm{C}\right)\end{array}$ & $\begin{array}{l}\text { Trans- } \\
\text { parency } \\
\text { (Secchi } \\
\text { disk) } \\
( \pm t)\end{array}$ & $\begin{array}{l}\text { Dissolved } \\
\text { oxygen } \\
(\mathrm{mg} / \mathrm{L})\end{array}$ & $\begin{array}{c}\text { Dissolved } \\
\text { oxygen } \\
\text { (percent } \\
\text { satura- } \\
\text { tion) }\end{array}$ \\
\hline \multicolumn{10}{|c|}{ October } \\
\hline 19 & 1422 & 0.9 & 300 & 553 & 7.6 & 19.9 & - - & 8.1 & 90 \\
\hline 19 & 1422 & 3.3 & 300 & 552 & 7.6 & 19.6 & $\ldots$ & 8.0 & 88 \\
\hline 19 & 1423 & 5.1 & 300 & 554 & 7.6 & 19.3 & $\ldots$ & 8.0 & 87 \\
\hline 19 & 1423 & 9.9 & 300 & 553 & 7.5 & 19.0 & - - & 7.9 & 86 \\
\hline 19 & 1424 & 15 & 300 & 553 & 7.5 & 18.8 & $\cdots$ & 7.7 & 83 \\
\hline 19 & 1424 & 20 & 300 & 549 & 7.5 & 18.7 & -- & 7.7 & 83 \\
\hline 19 & 1425 & 24 & 300 & 553 & 7.5 & 18.7 & $\cdots$ & 7.5 & 81 \\
\hline 19 & 1417 & 0.4 & 700 & 554 & 7.6 & 19.9 & - & 8.2 & 90 \\
\hline 19 & 1417 & 3.1 & 700 & 555 & 7.6 & 19.4 & 2.5 & 8.0 & 87 \\
\hline 19 & 1418 & 5.1 & 700 & 552 & 7.5 & 18.9 & - - & 7.7 & 84 \\
\hline 19 & 1418 & 10 & 700 & 554 & 7.5 & 18.9 & $\cdots$ & 7.7 & 83 \\
\hline 19 & 1419 & 15 & 700 & 555 & 7.5 & 18.8 & $\cdots$ & 7.6 & 83 \\
\hline 19 & 1419 & 20 & 700 & 553 & 7.5 & 18.7 & - & 7.5 & 82 \\
\hline 19 & 1420 & 25 & 700 & 547 & 7.5 & 18.6 & - - & 7.6 & 82 \\
\hline 19 & 1420 & 28 & 700 & 552 & 7.5 & 18.6 & $\cdots$ & 7.5 & 81 \\
\hline 19 & 1412 & 1.1 & 1,000 & 554 & 7.6 & 19.7 & $\cdots$ & 8.1 & 90 \\
\hline 19 & 1412 & 3.1 & 1,000 & 555 & 7.6 & 19.5 & - & 8.0 & 88 \\
\hline 19 & 1413 & 5.0 & 1.000 & 555 & 7.5 & 19.0 & $\cdots$ & 7.8 & 85 \\
\hline 19 & 1413 & 10 & 1,000 & 553 & 7.5 & 18.9 & -- & 7.6 & 83 \\
\hline 19 & 1414 & 15 & 1,000 & 555 & 7.5 & 18.9 & $\cdots$ & 7.6 & 83 \\
\hline 19 & 1414 & 20 & 1,000 & 555 & 7.5 & 18.9 & - & 7.6 & 82 \\
\hline
\end{tabular}


Table 18. Water-quality data for station 391559081341201, Ohio River at river mile 184.6, June to October, 1995.

[ft $=$ feet $; \mu \mathrm{S} / \mathrm{cm}=$ microsiemens per centımeter; ${ }^{\circ} \mathrm{C}=$ degrees Celsius: $\mathrm{mg} / \mathrm{L}=$ milligrams per liter;.. = data not collected]

\begin{tabular}{|c|c|c|c|c|c|c|c|c|c|}
\hline Date & Time & $\begin{array}{c}\text { Sampling } \\
\text { depth } \\
\text { (ft) }\end{array}$ & $\begin{array}{c}\text { Sample } \\
\text { location } \\
\text { (Et from } \\
\text { left bank) }\end{array}$ & $\begin{array}{l}\text { Specific } \\
\text { conduct } \\
\text { ance } \\
(\mu \mathrm{S} / \mathrm{cm})\end{array}$ & $\begin{array}{l}\text { pH } \\
\text { (stan- } \\
\text { dard } \\
\text { units) }\end{array}$ & $\begin{array}{c}\text { Temper- } \\
\text { ature, } \\
\text { water } \\
\left({ }^{\circ} \mathrm{C}\right)\end{array}$ & $\begin{array}{c}\text { Trans - } \\
\text { parency } \\
\text { (Secchl } \\
\text { disk) } \\
\text { (Et) }\end{array}$ & $\begin{array}{l}\text { Dissolved } \\
\text { oxygen } \\
(\mathrm{mg} / \mathrm{L})\end{array}$ & $\begin{array}{c}\text { Dissolved } \\
\text { oxygen } \\
\text { (percent } \\
\text { satura- } \\
\text { tion) }\end{array}$ \\
\hline
\end{tabular}

\begin{tabular}{|c|c|c|c|c|c|c|c|c|c|}
\hline \multicolumn{10}{|l|}{ June } \\
\hline 29 & 0435 & 0.2 & 300 & 406 & 7.5 & 27.3 & $\cdots$ & 7.1 & 91 \\
\hline 29 & 0434 & 2.9 & 300 & 406 & 7.5 & 27.3 & . & 7.1 & 91 \\
\hline 29 & 0434 & 5.7 & 300 & 408 & 7.5 & 27.3 & $\cdots$ & 7.1 & 91 \\
\hline 29 & 0433 & 10 & 300 & 407 & 7.5 & 27.3 & $\cdots$ & 7.0 & 91 \\
\hline 29 & 0432 & 15 & 300 & 406 & 7.5 & 27.3 & $\cdots$ & 7.1 & 92 \\
\hline 29 & 0432 & 20 & 300 & 405 & 7.5 & 27.3 & $\cdots$ & 7.1 & 91 \\
\hline 29 & 0431 & 26 & 300 & 397 & 7.4 & 27.3 & $\cdots$ & 7.0 & 90 \\
\hline 29 & 0431 & 30 & 300 & 378 & 7.4 & 27.1 & $\cdots$ & 6.9 & 89 \\
\hline 29 & 0430 & 36 & 300 & 341 & 7.3 & 26.6 & $\cdots$ & 6.8 & 86 \\
\hline 29 & 0437 & 0.3 & 600 & 431 & 7.6 & 27.4 & -- & 7.1 & 92 \\
\hline 29 & 0437 & 3.1 & 600 & 428 & 7.6 & 27.5 & $\cdots$ & 7.1 & 92 \\
\hline 29 & 0438 & 4.9 & 600 & 435 & 7.6 & 27.4 & $\cdots$ & 7.1 & 92 \\
\hline 29 & 0438 & 9.6 & 600 & 436 & 7.6 & 27.5 & $\cdots$ & 7.1 & 92 \\
\hline 29 & 0439 & 15 & 600 & 439 & 7.6 & 27.5 & -- & 7.1 & 92 \\
\hline 29 & 0439 & 20 & 600 & 431 & 7.6 & 27.4 & $\cdots$ & 7.1 & 92 \\
\hline 29 & 0440 & 25 & 600 & 417 & 7.6 & 27.3 & $\cdots$ & 7.1 & 91 \\
\hline 29 & 0440 & 30 & 600 & 382 & 7.5 & 27.0 & $\cdots$ & 7.1 & 90 \\
\hline 29 & 0441 & 35 & 600 & 383 & .7 .4 & 26.9 & $\cdots$ & 6.9 & 88 \\
\hline 29 & 0441 & 37 & 600 & 356 & 7.4 & 26.7 & $\cdots$ & 6.7 & 86 \\
\hline 29 & 0443 & 0.3 & 900 & 467 & 7.7 & 27.5 & $\cdots$ & 6.9 & 89 \\
\hline 29 & 0443 & 3.0 & 900 & 472 & 7.7 & 27.5 & -- & 6.8 & 88 \\
\hline 29 & 0444 & 5.1 & 900 & 470 & 7.7 & 27.5 & $\cdots$ & 6.8 & 88 \\
\hline 29 & 0444 & 9.5 & 900 & 474 & 7.7 & 27.5 & $\cdots$ & 6.8 & 88 \\
\hline 29 & 0445 & 15 & 900 & 469 & 7.7 & 27.5 & $\cdots$ & 6.8 & 88 \\
\hline 29 & 0445 & 18 & 900 & 471 & 7.7 & 27.5 & $\cdots$ & 6.8 & 88 \\
\hline \multicolumn{10}{|l|}{ July } \\
\hline 13 & 0443 & 0.4 & 300 & 492 & 8.1 & 27.7 & $\cdots$ & 8.7 & 112 \\
\hline 13 & 0442 & 3.1 & 300 & 491 & 8.0 & 27.7 & $\cdots$ & 8.6 & 112 \\
\hline 13 & 0442 & 5.2 & 300 & 491 & 8.0 & 27.7 & $\cdots$ & 8.7 & 112 \\
\hline 13 & 0441 & 9.9 & 300 & 492 & 8.0 & 27.7 & $\cdots$ & 8.7 & 11 \\
\hline 13 & 0440 & 15 & 300 & 490 & 7.9 & 27.6 & $\cdots$ & 8.2 & 10 \\
\hline 13 & 0440 & 20 & 300 & 483 & 7.8 & 27.5 & $\cdots$ & 7.9 & 102 \\
\hline 13 & 0439 & 26 & 300 & 475 & 7.7 & 27.4 & $\cdots$ & 7.8 & 101 \\
\hline 13 & 0439 & 30 & 300 & 468 & 7.7 & 27.4 & $\cdots$ & 7.8 & 100 \\
\hline 13 & 0438 & 36 & 300 & 278 & 7.4 & 25.9 & $\cdots$ & 5.9 & 74 \\
\hline
\end{tabular}


Table 18. Water-quality data for station 391559081341201, Ohio River at river mile 184.6, June to October, 1995, Continued.

[ft $=$ feet $; \mu \mathrm{s} / \mathrm{cm}=$ microsiemens per centımeter $;{ }^{\circ} \mathrm{C}=$ degrees Celsius; $\mathrm{mg} / \mathrm{L}=\mathrm{mili}$ igrams per liter; $\cdots$ = data not collected]

\begin{tabular}{|c|c|c|c|c|c|c|c|c|c|}
\hline Date & Time & $\begin{array}{l}\text { Sampling } \\
\text { depth } \\
\text { (ft) }\end{array}$ & $\begin{array}{l}\text { Sample } \\
\text { location } \\
\text { (ft from } \\
\text { left bank) }\end{array}$ & $\begin{array}{l}\text { Specific } \\
\text { conduct- } \\
\text { ance } \\
(\mu S / \mathrm{cm})\end{array}$ & $\begin{array}{l}\mathrm{pH} \\
\text { (stan- } \\
\text { dard } \\
\text { unlts) }\end{array}$ & $\begin{array}{c}\text { Temper- } \\
\text { ature, } \\
\text { water } \\
\left({ }^{\circ} \mathrm{C}\right)\end{array}$ & $\begin{array}{l}\text { Trans- } \\
\text { parency } \\
\text { (secchl } \\
\text { disk) } \\
\text { (ft) }\end{array}$ & $\begin{array}{l}\text { Dissolved } \\
\text { oxygen } \\
(\mathrm{mg} / \mathrm{L})\end{array}$ & $\begin{array}{c}\text { Dissolved } \\
\text { oxygen } \\
\text { (percent } \\
\text { satura- } \\
\text { tion) }\end{array}$ \\
\hline
\end{tabular}

\begin{tabular}{|c|c|c|c|c|}
\hline JulY & & & & \\
\hline 13 & 0515 & 0.4 & 600 & 4 \\
\hline 13 & 0514 & 3.2 & 600 & 49 \\
\hline 13 & 0514 & 5.3 & 600 & $y$ \\
\hline 13 & 0513 & 10 & 600 & \\
\hline 13 & 0513 & 15 & 600 & \\
\hline 13 & 0512 & 20 & 600 & 49 \\
\hline 13 & 0512 & 25 & 600 & \\
\hline 13 & 0511 & 30 & 600 & \\
\hline 13 & 0511 & 34 & 600 & \\
\hline 13 & 0452 & 0.5 & 900 & \\
\hline 13 & 0451 & 3.4 & 900 & \\
\hline 13 & 0451 & 5.1 & 900 & \\
\hline 13 & 0450 & 10 & 900 & \\
\hline 13 & 0450 & 15 & 900 & \\
\hline 13 & 0449 & 20 & 900 & \\
\hline 13 & 0449 & 26 & 900 & \\
\hline 13 & 1626 & 0.4 & 300 & \\
\hline 13 & 1626 & 3.0 & 300 & \\
\hline 13 & 1627 & 5.2 & 300 & 49 \\
\hline 13 & 1628 & 9.9 & 300 & 49 \\
\hline 13 & 1628 & 15 & 300 & \\
\hline 13 & 1629 & 20 & 300 & 4 \\
\hline 13 & 1629 & 25 & 300 & \\
\hline 13 & 1630 & 30 & 300 & 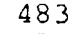 \\
\hline 13 & 1631 & 34 & 300 & \\
\hline 13 & 1619 & 0.9 & 600 & 4 \\
\hline 13 & 1618 & 3.0 & 600 & 4 \\
\hline 13 & 1618 & 5.2 & 600 & 4 \\
\hline 13 & 1617 & 10 & 600 & 4 \\
\hline 13 & 1616 & 15 & 600 & 4 \\
\hline 13 & 1616 & 20 & 600 & 9 \\
\hline 13 & 1615 & 25 & 600 & 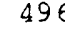 \\
\hline 13 & 1614 & 31 & 600 & \\
\hline 13 & 1614 & 34 & 600 & \\
\hline
\end{tabular}

\begin{tabular}{|c|c|c|c|c|}
\hline 8.1 & 27.7 & - & 8.7 & 112 \\
\hline 8.1 & 27.7 & -- & 8.7 & 112 \\
\hline 8.1 & 27.7 & $\cdots$ & 8.7 & 112 \\
\hline 8.0 & 27.7 & -- & 8.6 & 111 \\
\hline 8.0 & 27.6 & $\cdots$ & 8.3 & 108 \\
\hline 7.9 & 27.5 & $\cdots$ & 7.9 & 102 \\
\hline 7.8 & 27.4 & $\cdots$ & 7.7 & 99 \\
\hline 7.7 & 27.2 & $\cdots$ & 7.5 & 96 \\
\hline 7.5 & 26.6 & - & 6.5 & 83 \\
\hline 8.0 & 27.6 & $\cdots$ & 8.4 & 108 \\
\hline 8.0 & 27.6 & -- & 8.4 & 108 \\
\hline 8.0 & 27.6 & $\cdots$ & 8.4 & 108 \\
\hline 8.0 & 27.6 & $\cdots$ & 8.3 & 108 \\
\hline 7.9 & 27.6 & - & 8.3 & 108 \\
\hline 7.9 & 27.5 & - & 8.2 & 106 \\
\hline 7.6 & 27.1 & $\cdots$ & 7.2 & 92 \\
\hline 8.7 & 30.0 & - & 12.8 & 172 \\
\hline 8.7 & 29.6 & $\cdots$ & 12.8 & 170 \\
\hline 8.5 & 29.1 & - & 11.5 & 153 \\
\hline 8.2 & 28.0 & -- & 11.1 & 144 \\
\hline 8.0 & 27.6 & $\cdots$ & 8.4 & 109 \\
\hline 7.9 & 27.5 & $\cdots$ & 8.3 & 107 \\
\hline 7.9 & 27.5 & $\cdots$ & 8.2 & 106 \\
\hline 7.9 & 27.5 & $\cdots$ & 8.1 & 105 \\
\hline 7.9 & 27.5 & $\cdots$ & 8.1 & 104 \\
\hline 8.5 & 29.2 & $\cdots$ & 11.2 & 149 \\
\hline 8.5 & 29.1 & $\cdots$ & 11.6 & 153 \\
\hline 8.4 & 28.7 & $\cdots$ & 10.5 & 138 \\
\hline 8.0 & 27.7 & - & 8.4 & 109 \\
\hline 7.9 & 27.6 & $\cdots$ & 8.2 & 105 \\
\hline 7.9 & 27.5 & $\cdots$ & 8.1 & 105 \\
\hline 7.9 & 27.5 & $\cdots$ & 8.1 & 105 \\
\hline 7.9 & 27.5 & - & 8.0 & 103 \\
\hline 7.5 & 26.4 & - & 6.3 & 79 \\
\hline
\end{tabular}


Table 18. Water-quality data for station 391559081341201, Ohio River at river mile 184.6, June to October, 1995, Continued.

[ft $=$ feet $; \mu \mathrm{s} / \mathrm{cm}=$ microsiemens per centimeter $;{ }^{\circ} \mathrm{C}=$ degrees Celsius;

$\mathrm{mg} / \mathrm{L}=$ milligrams per liter; - = data not collected]

\begin{tabular}{|c|c|c|c|c|c|c|c|c|c|}
\hline Date & Time & $\begin{array}{l}\text { Sampling } \\
\text { depth } \\
\text { (ft) }\end{array}$ & $\begin{array}{c}\text { Sample } \\
\text { location } \\
\text { (Et from } \\
\text { left bank) }\end{array}$ & $\begin{array}{l}\text { Specific } \\
\text { conduct- } \\
\text { ance } \\
(\mu \mathrm{s} / \mathrm{cm})\end{array}$ & $\begin{array}{l}\text { pH } \\
\text { (stan- } \\
\text { dard } \\
\text { units) }\end{array}$ & $\begin{array}{c}\text { Temper- } \\
\text { ature, } \\
\text { water } \\
\left({ }^{\circ} \mathrm{C}\right)\end{array}$ & $\begin{array}{c}\text { Trans- } \\
\text { parency } \\
\text { (secchi } \\
\text { disk) } \\
\text { (Et) }\end{array}$ & $\begin{array}{l}\text { Dissolved } \\
\text { oxygen } \\
(\mathrm{mg} / \mathrm{L})\end{array}$ & $\begin{array}{c}\text { Dissolved } \\
\text { oxygen } \\
\text { (percent } \\
\text { satura- } \\
\text { tion) }\end{array}$ \\
\hline
\end{tabular}

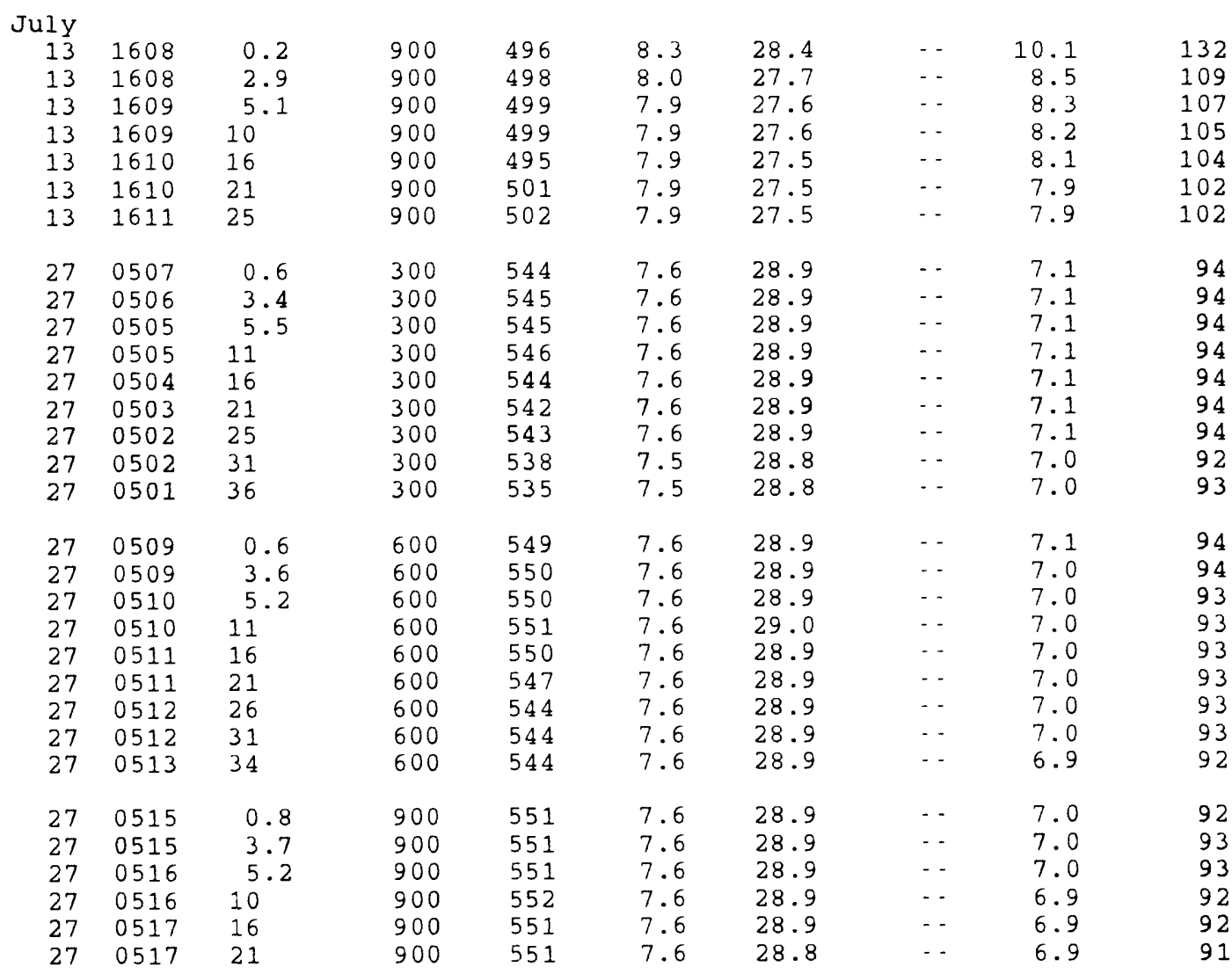


Table 18. Water-quality data for station 391559081341201, Ohio River at river mile 184.6, June to October, 1995, Continued.

[ft $=$ feet; $\mu \mathrm{S} / \mathrm{cm}=$ microsiemens per centimeter; ${ }^{\circ} \mathrm{C}=$ degrees Celsius;

$\mathrm{mg} / \mathrm{L}=$ militigrams per liter; $-\cdot$ = data not collected]

\begin{tabular}{|c|c|c|c|c|c|c|c|c|c|}
\hline Date & Time & $\begin{array}{l}\text { Sampling } \\
\text { depth } \\
(f t)\end{array}$ & $\begin{array}{c}\text { Sample } \\
\text { location } \\
\text { (ft from } \\
\text { left bank) }\end{array}$ & $\begin{array}{l}\text { Specitic } \\
\text { conduct. } \\
\text { ance } \\
(\mu \mathrm{S} / \mathrm{cm})\end{array}$ & $\begin{array}{l}\mathrm{pH} \\
\text { (stan- } \\
\text { dard } \\
\text { units) }\end{array}$ & $\begin{array}{c}\text { Temper- } \\
\text { ature, } \\
\text { water } \\
\left({ }^{\circ} \mathrm{C}\right)\end{array}$ & $\begin{array}{l}\text { Trans- } \\
\text { parency } \\
\text { (SecchI } \\
\text { disk) } \\
(\mathrm{ft})\end{array}$ & $\begin{array}{l}\text { Dissolved } \\
\text { oxygen } \\
(\mathrm{mg} / \mathrm{L})\end{array}$ & $\begin{array}{c}\text { Dissolved } \\
\text { oxygen } \\
\text { (percent } \\
\text { satura- } \\
\text { tion) }\end{array}$ \\
\hline
\end{tabular}

\begin{tabular}{|c|c|c|c|c|c|c|c|c|c|}
\hline $\begin{array}{r}\text { July } \\
27\end{array}$ & 1400 & 0.5 & 300 & 544 & 78 & 30.0 & $\ldots$ & 78 & 105 \\
\hline 27 & 1400 & 3.1 & 300 & 543 & 7.7 & 29.3 & . & 7.3 & 97 \\
\hline 27 & 1401 & 4.9 & 300 & 541 & 7.6 & 29.2 & $\cdots$ & 7.1 & 94 \\
\hline 27 & 1401 & 10 & 300 & 542 & 7.6 & 29.1 & $\ldots$ & 7.0 & 93 \\
\hline 27 & 1402 & 15 & 300 & 542 & 7.6 & 29.1 & $\cdots$ & 7.0 & 93 \\
\hline 27 & 1402 & 20 & 300 & 541 & 7.6 & 29.1 & $\cdots$ & 6.9 & 92 \\
\hline 27 & 1403 & 25 & 300 & 541 & 7.6 & 29.1 & $\cdots$ & 6.9 & 91 \\
\hline 27 & 1403 & 30 & 300 & 542 & 7.6 & 29.1 & $\cdots$ & 6.9 & 91 \\
\hline 27 & 1404 & 35 & 300 & 544 & 7.6 & 29.1 & $\cdots$ & 6.9 & 91 \\
\hline 27 & 1404 & 37 & 300 & 540 & 7.6 & 29.1 & - & 6.9 & 91 \\
\hline 27 & 1407 & 0.6 & 600 & 544 & 7.7 & 29.7 & - & 7.4 & 99 \\
\hline 27 & 1407 & 3.1 & 600 & 547 & 7.6 & 29.3 & - & 7.1 & 94 \\
\hline 27 & 1408 & 5.0 & 600 & 545 & 7.6 & 29.2 & 3.5 & 6.9 & 91 \\
\hline 27 & 1408 & 10 & 600 & 551 & 7.6 & 29.2 & $\ldots$ & 6.9 & 91 \\
\hline 27 & 1409 & 15 & 600 & 552 & 7.6 & 29.2 & $\cdots$ & 6.8 & 90 \\
\hline 27 & 1409 & 20 & 600 & 554 & 7.6 & 29.2 & $\cdots$ & 6.8 & 90 \\
\hline 27 & 1410 & 25 & 600 & 554 & 7.6 & 29.2 & $\cdots$ & 6.8 & 90 \\
\hline 27 & 1411 & 30 & 600 & 542 & 7.5 & 29.1 & - & 6.8 & 90 \\
\hline 27 & 1411 & 31 & 600 & 554 & 7.5 & 29.1 & - & 6.8 & 90 \\
\hline 27 & 1413 & 0.3 & 900 & 549 & 7.7 & 30.3 & $\cdots$ & 7.4 & 100 \\
\hline 27 & 1413 & 3.3 & 900 & 549 & 7.6 & 29.3 & $\cdots$ & 7.1 & 94 \\
\hline 27 & 1416 & 5.0 & 900 & 549 & 7.7 & 29.4 & - & 7.2 & 96 \\
\hline 27 & 1414 & 10 & 900 & 545 & 7.6 & 29.2 & - & 6.8 & 90 \\
\hline 27 & 1414 & 15 & 900 & 551 & 7.6 & 29.2 & $\cdots$ & 6.8 & 90 \\
\hline 27 & 1415 & 20 & 900 & 547 & 7.6 & 29.2 & $\cdots$ & 6.8 & 90 \\
\hline 27 & 1415 & 25 & 900 & 549 & 7.5 & 29.1 & $\cdots$ & 6.8 & 90 \\
\hline Augu & & & & & & & & & \\
\hline 10 & 1541 & 1.1 & 300 & 407 & 7.6 & 25.9 & - & 6.7 & 84 \\
\hline 10 & 1541 & 3.6 & 300 & 402 & 7.5 & 25.6 & $\cdots$ & 6.6 & 82 \\
\hline 10 & 1542 & 5.7 & 300 & 403 & 7.5 & 25.6 & $\cdots$ & 6.6 & 82 \\
\hline 10 & 1542 & 10 & 300 & 403 & 7.5 & 25.6 & $\cdots$ & 6.5 & 81 \\
\hline 10 & 1543 & 15 & 300 & 399 & 7.5 & 25.6 & $\cdots$ & 6.5 & 81 \\
\hline 10 & 1543 & 20 & 300 & 395 & 7.5 & 25.6 & $\cdots$ & 6.5 & 81 \\
\hline 10 & 1544 & 25 & 300 & 375 & 7.5 & 25.3 & $\cdots$ & 6.5 & 81 \\
\hline 10 & 1544 & 30 & 300 & 364 & 7.5 & 25.3 & $\cdots$ & 6.5 & 81 \\
\hline 10 & 1545 & 35 & 300 & 361 & 7.5 & 25.2 & $\cdots$ & 6.5 & 81 \\
\hline
\end{tabular}


Table 18. Water-quality data for station 391559081341201, Ohio River at river mile 184.6, June to October, 1995, Continued.

[ft = feet; $\mu \mathrm{s} / \mathrm{cm}=$ microsiemens per centimeter; ${ }^{\circ} \mathrm{C}=$ degrees Celsius; $\mathrm{mg} / \mathrm{L}=$ milligrams per liter;.. = data not collected]

\begin{tabular}{|c|c|c|c|c|c|c|c|c|c|}
\hline Date & Time & $\begin{array}{l}\text { Sampling } \\
\text { depth } \\
\text { (ft) }\end{array}$ & $\begin{array}{c}\text { Sample } \\
\text { location } \\
\text { (ft from } \\
\text { left bank) }\end{array}$ & $\begin{array}{l}\text { Specif } 1 \text { c } \\
\text { conduct- } \\
\text { ance } \\
(\mu S / c m)\end{array}$ & $\begin{array}{l}\mathrm{pH} \\
\text { (stan- } \\
\text { dard } \\
\text { units) }\end{array}$ & $\begin{array}{c}\text { Temper- } \\
\text { ature, } \\
\text { water } \\
\left({ }^{\circ} \mathrm{C}\right)\end{array}$ & $\begin{array}{c}\text { Trans- } \\
\text { parency } \\
\text { (Secchi } \\
\text { disk) } \\
(t t)\end{array}$ & $\begin{array}{c}\text { Dissolved } \\
\text { oxygen } \\
\text { (mg/L) }\end{array}$ & $\begin{array}{c}\text { Dissolved } \\
\text { oxygen } \\
\text { (percent } \\
\text { satura- } \\
\text { tion) }\end{array}$ \\
\hline
\end{tabular}

\begin{tabular}{|c|c|c|c|c|c|c|c|c|c|}
\hline \multicolumn{10}{|c|}{ August } \\
\hline 10 & 1535 & 1.4 & 600 & 409 & 7.6 & 25.9 & $<0.5$ & 6.6 & 83 \\
\hline 10 & 1535 & 3.6 & 600 & 408 & 7.5 & 25.8 & $\ldots$ & 6.6 & 82 \\
\hline 10 & 1536 & 5.6 & 600 & 406 & 7.5 & 25.8 & $\cdots$ & 6.5 & 82 \\
\hline 10 & 1536 & 10 & 600 & 407 & 7.5 & 25.7 & $\cdots$ & 6.5 & 81 \\
\hline 10 & 1537 & 15 & 600 & 406 & 7.5 & 25.7 & - & 6.5 & 81 \\
\hline 10 & 1537 & 20 & 600 & 404 & 7.5 & 25.7 & . & 6.5 & 81 \\
\hline 10 & 1538 & 25 & 600 & 402 & 7.5 & 25.7 & - . & 6.5 & 81 \\
\hline 10 & 1538 & 30 & 600 & 389 & 7.5 & 25.5 & $\cdots$ & 6.5 & 80 \\
\hline 10 & 1539 & 35 & 600 & 371 & 7.5 & 25.2 & $\cdot-$ & 6.4 & 80 \\
\hline 10 & 1539 & 37 & 600 & 327 & 7.5 & 24.9 & $\cdots$ & 6.4 & 79 \\
\hline 10 & 1530 & 1.2 & 900 & 383 & 7.5 & 25.8 & $\cdots$ & 6.6 & 82 \\
\hline 10 & 1530 & 3.9 & 900 & 406 & 7.5 & 25.7 & $\cdots$ & 6.5 & 82 \\
\hline 10 & 1531 & 5.7 & 900 & 406 & 7.5 & 25.6 & - & 6.5 & 81 \\
\hline 10 & 1531 & 10 & 900 & 406 & 7.5 & 25.6 & - & 6.5 & 81 \\
\hline 10 & 1532 & 15 & 900 & 405 & 7.5 & 25.6 & -- & 6.5 & 81 \\
\hline 10 & 1532 & 20 & 900 & 406 & 7.5 & 25.6 & $\cdots$ & 6.5 & 81 \\
\hline 10 & 1533 & 25 & 900 & 406 & 7.5 & 25.6 & $\cdots$ & 6.5 & 81 \\
\hline 10 & 1533 & 27 & 900 & 406 & 7.5 & 25.6 & $\cdots$ & 6.4 & 80 \\
\hline 24 & 0535 & 0.7 & 300 & 550 & 7.9 & 29.4 & $\cdots$ & 7.5 & 100 \\
\hline 24 & 0535 & 2.8 & 300 & 546 & 8.0 & 29.4 & $\cdots$ & 7.5 & 100 \\
\hline 24 & 0536 & 4.9 & 300 & 545 & 8.0 & 29.4 & - & 7.5 & 100 \\
\hline 24 & 0536 & 10 & 300 & 545 & 8.0 & 29.4 & $\cdots$ & 7.5 & 100 \\
\hline 24 & 0537 & 15 & 300 & 552 & 8.0 & 29.4 & $\cdots$ & 7.5 & 100 \\
\hline 24 & 0537 & 20 & 300 & 546 & 8.0 & 29.4 & $\cdots$ & 7.5 & 100 \\
\hline 24 & 0538 & 25 & 300 & 543 & 8.0 & 29.4 & $\cdots$ & 7.5 & 100 \\
\hline 24 & 0538 & 30 & 300 & 551 & 8.0 & 29.4 & $\cdots$ & 7.5 & 99 \\
\hline 24 & 0539 & 35 & 300 & 381 & 7.5 & 28.4 & $-\cdot$ & 5.3 & 69 \\
\hline 24 & 0539 & 38 & 300 & 345 & 7.4 & 28.2 & $\cdots$ & 5.3 & 69 \\
\hline 24 & 0542 & 0.5 & 600 & 553 & 8.0 & 29.3 & $\cdots$ & 7.5 & 99 \\
\hline 24 & 0542 & 2.9 & 600 & 540 & 7.9 & 29.4 & - - & 7.4 & 98 \\
\hline 24 & 0543 & 5.1 & 600 & 546 & 7.9 & 29.4 & $\cdots$ & 7.4 & 98 \\
\hline 24 & 0543 & 10 & 600 & 552 & 7.9 & 29.4 & $\cdots$ & 7.4 & 99 \\
\hline 24 & 0544 & 15 & 600 & 554 & 7.9 & 29.4 & $\cdots$ & 7.4 & 98 \\
\hline 24 & 0544 & 20 & 600 & 550 & 7.9 & 29.4 & $\cdots$ & 7.4 & 99 \\
\hline 24 & 0545 & 25 & 600 & 550 & 7.9 & 29.4 & $\cdots$ & 7.6 & 101 \\
\hline
\end{tabular}


Table 18. Water-quality data for station 391559081341201, Ohio River at river mile 184.6, June to October, 1995, Continued.

[ft $=$ feet $; \mu \mathrm{s} / \mathrm{cm}=$ microsıemens per centimeter $;{ }^{\circ} \mathrm{C}=$ degrees Celsius;

$\mathrm{mg} / \mathrm{L}=\mathrm{milligrams}$ per liter;. = data not collected]

\begin{tabular}{|c|c|c|c|c|c|c|c|c|c|}
\hline Date & Time & $\begin{array}{l}\text { Sampling } \\
\text { depth } \\
\text { (ft) }\end{array}$ & $\begin{array}{c}\text { Sample } \\
\text { location } \\
\text { (Et from } \\
\text { left bank) }\end{array}$ & $\begin{array}{c}\text { Specieic } \\
\text { conduct - } \\
\text { ance } \\
(\mu \mathrm{s} / \mathrm{cm})\end{array}$ & $\begin{array}{l}\mathrm{pH} \\
\text { (stan- } \\
\text { dard } \\
\text { units) }\end{array}$ & $\begin{array}{c}\text { Temper- } \\
\text { ature, } \\
\text { water } \\
\left({ }^{\circ} \mathrm{C}\right)\end{array}$ & $\begin{array}{l}\text { Trans - } \\
\text { parency } \\
\text { (secchl } \\
\text { disk) } \\
\text { (ft) }\end{array}$ & $\begin{array}{c}\text { Dissolved } \\
\text { oxygen } \\
\text { (mg/L) }\end{array}$ & $\begin{array}{c}\text { Dissolved } \\
\text { oxygen } \\
\text { (percent } \\
\text { satura- } \\
\text { tion) }\end{array}$ \\
\hline
\end{tabular}

\begin{tabular}{|c|c|c|c|c|c|c|c|c|c|}
\hline ugu & & & & & & & & & \\
\hline 24 & 0549 & 0.9 & 900 & 551 & 7.9 & 29.3 & - & 7.2 & 96 \\
\hline 24 & 0550 & 3.1 & 900 & 544 & 7.9 & 29.3 & - & 7.4 & 98 \\
\hline 24 & 0550 & 5.0 & 900 & 556 & 7.9 & 29.3 & $\cdots$ & 7.4 & 98 \\
\hline 24 & 0551 & 10 & 900 & 543 & 7.9 & 29.3 & - - & 7.4 & 98 \\
\hline 24 & 0551 & 15 & 900 & 540 & 7.9 & 29.3 & - - & 7.4 & 98 \\
\hline 24 & 0552 & 20 & 900 & 543 & 7.9 & 29.3 & $\cdots$ & 7.1 & 95 \\
\hline 24 & 1309 & 0.4 & 300 & 545 & 8.3 & 30.7 & $\cdots$ & 9.5 & 130 \\
\hline 24 & 1309 & 3.1 & 300 & 544 & 8.2 & 29.7 & - - & 9.2 & 123 \\
\hline 24 & 1310 & 5.2 & 300 & 546 & 8.0 & 29.3 & $\cdots$ & 7.9 & 105 \\
\hline 24 & 1311 & 10 & 300 & 547 & 7.9 & 29.2 & - & 7.3 & 97 \\
\hline 24 & 1311 & 15 & 300 & 549 & 7.9 & 29.2 & $\cdots$ & 7.2 & 95 \\
\hline 24 & 1312 & 20 & 300 & 546 & 7.8 & 29.2 & $\cdots$ & 7.2 & 95 \\
\hline 24 & 1313 & 25 & 300 & 543 & 7.8 & 29.1 & $\cdots$ & 6.9 & 92 \\
\hline 24 & 1313 & 30 & 300 & 540 & 7.8 & 29.1 & $\cdots$ & 6.9 & 91 \\
\hline 24 & 1314 & 35 & 300 & 537 & 7.8 & 29.1 & - & 7.0 & 92 \\
\hline 24 & 1315 & 37 & 300 & 537 & 7.8 & 29.1 & - - & 6.8 & 90 \\
\hline 24 & 1317 & 0.4 & 600 & 546 & 8.3 & 30.4 & $\cdots$ & 9.7 & 131 \\
\hline 24 & 1317 & 3.3 & 600 & 547 & 8.0 & 29.3 & 2.5 & 7.9 & 105 \\
\hline 24 & 1318 & 5.4 & 600 & 550 & 7.9 & 29.3 & - - & 7.5 & 100 \\
\hline 24 & 1319 & 10 & 600 & 547 & 7.9 & 29.2 & - & 7.4 & 98 \\
\hline 24 & 1319 & 15 & 600 & 548 & 7.8 & 29.2 & $\cdots$ & 7.3 & 96 \\
\hline 24 & 1320 & 20 & 600 & 547 & 7.8 & 29.1 & $\cdots$ & 7.1 & 94 \\
\hline 24 & 1320 & 25 & 600 & 548 & 7.8 & 29.1 & $\cdots$ & 7.1 & 93 \\
\hline 24 & 1321 & 30 & 600 & 546 & 7.8 & 29.1 & - & 7.1 & 94 \\
\hline 24 & 1323 & 0.4 & 900 & 545 & 8.3 & 30.4 & $\cdots$ & 9.7 & 132 \\
\hline 24 & 1323 & 3.2 & 900 & 549 & 8.0 & 29.3 & - & 7.8 & 104 \\
\hline 24 & 1324 & 5.2 & 900 & 550 & 7.9 & 29.2 & -- & 7.3 & 97 \\
\hline 24 & 1324 & 9.9 & 900 & 549 & 7.8 & 29.2 & $\cdots$ & 7.1 & \\
\hline 24 & 1325 & 15 & 900 & 546 & 7.8 & 29.1 & $\cdots$ & 7.1 & \\
\hline 24 & 1325 & 20 & 900 & 548 & 7.8 & 29.1 & - - & 7.0 & \\
\hline 24 & 1326 & 24 & 900 & 546 & 7.8 & 29.1 & $\cdots$ & 6.7 & \\
\hline
\end{tabular}


Table 18. Water-quality data for station 391559081341201, Ohio River at river mile 184.6, June to October, 1995, Continued.

ft $=$ feet $\mu \mu \mathrm{S} / \mathrm{cm}=$ microsiemens per centimeter $;{ }^{\circ} \mathrm{C}=$ degrees Celsius; $\mathrm{mg} / \mathrm{L}=\mathrm{milligrams}$ per liter; . - = data not collected]

\begin{tabular}{|c|c|c|c|c|c|c|c|c|c|}
\hline Date & Time & $\begin{array}{l}\text { Sampling } \\
\text { depth } \\
(f t)\end{array}$ & $\begin{array}{c}\text { Sample } \\
\text { location } \\
\text { (ft from } \\
\text { left bank) }\end{array}$ & $\begin{array}{l}\text { Specitic } \\
\text { conduct- } \\
\text { ance } \\
(\mu \mathrm{s} / \mathrm{cm})\end{array}$ & $\begin{array}{l}\mathrm{pH} \\
\text { (stan- } \\
\text { dard } \\
\text { units) }\end{array}$ & $\begin{array}{c}\text { Temper- } \\
\text { ature, } \\
\text { water } \\
\left({ }^{\circ} \mathrm{C}\right)\end{array}$ & $\begin{array}{c}\text { Trans- } \\
\text { parency } \\
\text { (Secchl } \\
\text { disk) } \\
\text { (tt) }\end{array}$ & $\begin{array}{l}\text { Dissolved } \\
\text { oxygen } \\
(\mathrm{mg} / \mathrm{L})\end{array}$ & $\begin{array}{c}\text { Dissolved } \\
\text { oxygen } \\
\text { (percent } \\
\text { satura- } \\
\text { tion) }\end{array}$ \\
\hline
\end{tabular}

\begin{tabular}{|c|c|c|c|c|c|c|c|c|c|}
\hline \multicolumn{10}{|c|}{ September } \\
\hline 06 & 0457 & 0.5 & 300 & 548 & 7.8 & 27.3 & $\cdots$ & 7.7 & 98 \\
\hline 06 & 0457 & 2.9 & 300 & 544 & 7.8 & 27.3 & $\cdots$ & 7.6 & 97 \\
\hline 06 & 0458 & 5.9 & 300 & 546 & 7.8 & 27.4 & $\cdots$ & 7.6 & 97 \\
\hline 06 & 0458 & 10 & 300 & 545 & 7.8 & 27.4 & $\cdots$ & 7.6 & 97 \\
\hline 06 & 0459 & 15 & 300 & 545 & 7.8 & 27.3 & $\cdots$ & 7.6 & 97 \\
\hline 06 & 0459 & 20 & 300 & 545 & 7.8 & 27.3 & $\cdots$ & 7.5 & 96 \\
\hline 06 & 0449 & 1.1 & 600 & 544 & 7.8 & 27.3 & $\cdots$ & 7.7 & 98 \\
\hline 06 & 0450 & 3.3 & 600 & 546 & 7.8 & 27.4 & - - & 7.6 & 97 \\
\hline 06 & 0452 & 5.0 & 600 & 547 & 7.8 & 27.4 & $\cdots$ & 7.6 & 98 \\
\hline 06 & 0450 & 10 & 600 & 547 & 7.8 & 27.4 & - & 7.7 & 98 \\
\hline 06 & 0451 & 15 & 600 & 546 & 7.8 & 27.4 & $\cdots$ & 7.6 & 98 \\
\hline 06 & 0452 & 20 & 600 & 543 & 7.8 & 27.4 & $\cdots$ & 7.6 & 97 \\
\hline 06 & 0453 & 25 & 600 & 539 & 7.7 & 27.4 & $\cdots$ & 7.5 & 96 \\
\hline 06 & 0455 & 30 & 600 & 535 & 7.7 & 27.3 & - & 7.4 & 95 \\
\hline 06 & 0454 & 35 & 600 & 540 & 7.7 & 27.4 & $\cdots$ & 7.5 & 96 \\
\hline 06 & 0454 & 38 & 600 & 535 & 7.8 & 27.4 & $\cdots$ & 7.4 & 95 \\
\hline 06 & 0443 & 1.2 & 900 & 544 & 7.8 & 27.3 & $\cdots$ & 7.7 & 98 \\
\hline 06 & 0443 & 3.4 & 900 & 545 & 7.8 & 27.4 & $\cdots$ & 7.7 & 99 \\
\hline 06 & 0444 & 4.9 & 900 & 545 & 7.8 & 27.4 & $\cdots$ & 7.8 & 100 \\
\hline 06 & 0444 & 10 & 900 & 545 & 7.8 & 27.4 & $\cdots$ & 7.7 & 99 \\
\hline 06 & 0445 & 15 & 900 & 546 & 7.8 & 27.4 & $\cdots$ & 7.7 & 98 \\
\hline 06 & 0445 & 20 & 900 & 544 & 7.8 & 27.4 & $\cdots$ & 7.7 & 98 \\
\hline 06 & 0446 & 25 & 900 & 547 & 7.8 & 27.4 & $\cdots$ & 7.6 & 98 \\
\hline 06 & 0446 & 30 & 900 & 546 & 7.8 & 27.4 & $-\cdot$ & 7.6 & 97 \\
\hline 06 & 0447 & 34 & 900 & 545 & 7.8 & 27.3 & $\cdots$ & 7.7 & 99 \\
\hline 06 & 1421 & 0.4 & 300 & 547 & 7.9 & 28.0 & $\cdots$ & 8.9 & 115 \\
\hline 06 & 1421 & 2.8 & 300 & 548 & 7.9 & 27.7 & - & 8.4 & 108 \\
\hline 06 & 1422 & 5.0 & 300 & 548 & 7.7 & 27.4 & $\cdots$ & 7.5 & 96 \\
\hline 06 & 1422 & 9.6 & 300 & 549 & 7.7 & 27.3 & $\cdots$ & 7.3 & 93 \\
\hline 06 & 1423 & 15 & 300 & 547 & 7.6 & 27.3 & - & 7.4 & 94 \\
\hline 06 & 1423 & 19 & 300 & 549 & 7.6 & 27.3 & -- & 7.1 & 91 \\
\hline 06 & 1424 & 25 & 300 & 543 & 7.6 & 27.2 & $\cdots$ & 7.1 & 90 \\
\hline 06 & 1424 & 30 & 300 & 541 & 7.6 & 27.2 & $\cdots$ & 7.1 & 90 \\
\hline 06 & 1425 & 35 & 300 & 540 & 7.6 & 27.2 & $\cdots$ & 7.0 & 89 \\
\hline 06 & 1425 & 36 & 300 & 542 & 7.6 & 27.2 & $\cdots$ & 6.8 & 87 \\
\hline
\end{tabular}


Table 18. Water-quality data for station 391559081341201, Ohio River at river mile 184.6, June to October, 1995, Continued.

[Et $=$ Eeet; $\mu \mathrm{S} / \mathrm{cm}=$ microsiemens per centimeter $;{ }^{\circ} \mathrm{C}=$ degrees Celsius;

$\mathrm{mg} / \mathrm{L}=\mathrm{milligrams}$ per liter; . . = data not collected]

\begin{tabular}{|c|c|c|c|c|c|c|c|c|c|}
\hline Date & Time & $\begin{array}{l}\text { Sampling } \\
\text { depth } \\
\text { (Et) }\end{array}$ & $\begin{array}{c}\text { Sample } \\
\text { location } \\
\text { (ft from } \\
\text { left bank) }\end{array}$ & $\begin{array}{l}\text { Specific } \\
\text { conduct. } \\
\text { ance } \\
(\mu \mathrm{S} / \mathrm{cm})\end{array}$ & $\begin{array}{l}\mathrm{pH} \\
\text { (stan- } \\
\text { dard } \\
\text { units) }\end{array}$ & $\begin{array}{c}\text { Temper- } \\
\text { ature. } \\
\text { water } \\
\left({ }^{\circ} \mathrm{C}\right)\end{array}$ & $\begin{array}{l}\text { Trans- } \\
\text { parency } \\
\text { (Secchi } \\
\text { disk) } \\
\text { (Et) }\end{array}$ & $\begin{array}{l}\text { Dissolved } \\
\text { oxygen } \\
(\mathrm{mg} / \mathrm{L})\end{array}$ & $\begin{array}{c}\text { Dissolved } \\
\text { oxygen } \\
\text { (percent } \\
\text { satura- } \\
\text { tion) }\end{array}$ \\
\hline \multicolumn{10}{|c|}{ September } \\
\hline 06 & 1415 & 0.5 & 600 & 549 & 8.1 & 28.6 & $\cdots$ & 9.1 & 119 \\
\hline 06 & 1415 & 2.9 & 600 & 548 & 7.8 & 27.6 & 2.0 & 8.1 & 104 \\
\hline 06 & 1416 & 4.9 & 600 & 547 & 7.7 & 27.5 & $\therefore$ & 7.6 & 98 \\
\hline 06 & 1416 & 9.5 & 600 & 546 & 7.7 & 27.4 & -- & 7.5 & 96 \\
\hline 06 & 1417 & 15 & 600 & 549 & 7.7 & 27.3 & $\cdots$ & 7.4 & 94 \\
\hline 06 & 1417 & 20 & 600 & 547 & 7.6 & 27.3 & $\cdots$ & 7.1 & 91 \\
\hline 06 & 1418 & 25 & 600 & 545 & 7.6 & 27.2 & $\cdots$ & 7.0 & 90 \\
\hline 06 & 1418 & 30 & 600 & 547 & 7.6 & 27.2 & $\cdots$ & 7.0 & 89 \\
\hline 06 & 1419 & 34 & 600 & 545 & 7.6 & 27.2 & $\cdots$ & 7.0 & 89 \\
\hline 06 & 1410 & 0.2 & 900 & 546 & 8.1 & 28.4 & $\cdots$ & 9.5 & 123 \\
\hline 06 & 1410 & 2.8 & 900 & 546 & 8.1 & 28.3 & - - & 9.5 & 124 \\
\hline 06 & 1411 & 4.8 & 900 & 545 & 8.1 & 28.0 & - & 9.4 & 122 \\
\hline 06 & 1411 & 9.8 & 900 & 547 & 7.6 & 27.3 & $\cdots$ & 7.3 & 93 \\
\hline 06 & 1412 & 15 & 900 & 549 & 7.6 & 27.3 & $\cdots$ & 7.2 & 92 \\
\hline 06 & 1412 & 20 & 900 & 549 & 7.6 & 27.2 & -- & 7.1 & 90 \\
\hline 06 & 1413 & 22 & 900 & 550 & 7.6 & 27.2 & -- & 6.8 & 87 \\
\hline 20 & 0543 & 0.5 & 300 & 673 & 7.5 & 23.9 & $\cdots$ & 6.4 & 77 \\
\hline 20 & 0542 & 3.1 & 300 & 672 & 7.5 & 23.9 & $\cdots$ & 6.3 & 76 \\
\hline 20 & 0542 & 5.1 & 300 & 675 & 7.5 & 23.9 & - & 6.3 & 76 \\
\hline 20 & 0541 & 10 & 300 & 671 & 7.5 & 23.9 & - - & 6.3 & 76 \\
\hline 20 & 0540 & 15 & 300 & 671 & 7.5 & 23.9 & $\cdots$ & 6.3 & 76 \\
\hline 20 & 0540 & 20 & 300 & 675 & 7.5 & 23.9 & $\cdots$ & 6.2 & 75 \\
\hline 20 & 0539 & 25 & 300 & 677 & 7.4 & 23.9 & $\cdots$ & 6.3 & 76 \\
\hline 20 & 0538 & 30 & 300 & 650 & 7.4 & 23.8 & -- & 6.1 & 74 \\
\hline 20 & 0538 & 36 & 300 & 533 & 7.3 & 23.5 & $\cdots$ & 5.6 & 68 \\
\hline 20 & 0552 & 0.6 & 600 & 674 & 7.5 & 23.9 & $\cdots$ & 6.4 & 77 \\
\hline 20 & 0551 & 3.3 & 600 & 672 & 7.5 & 23.9 & $\cdots$ & 6.5 & 79 \\
\hline 20 & 0550 & 5.0 & 600 & 675 & 7.5 & 23.9 & - & 6.5 & 79 \\
\hline 20 & 0549 & 9.9 & 600 & 675 & 7.5 & 23.9 & $\cdots$ & 6.4 & 78 \\
\hline 20 & 0549 & 15 & 600 & 675 & 7.5 & 23.9 & - & 6.4 & 78 \\
\hline 20 & 0548 & 20 & 600 & 675 & 7.5 & 23.9 & - & 6.4 & 78 \\
\hline 20 & 0547 & 25 & 600 & 676 & 7.5 & 23.9 & - & 6.6 & 80 \\
\hline 20 & 0546 & 31 & 600 & 674 & 7.5 & 23.9 & - & 6.3 & 77 \\
\hline 20 & 0546 & 32 & 600 & 675 & 7.5 & 23.9 & $\cdots$ & 6.4 & 78 \\
\hline
\end{tabular}


Table 18. Water-quality data for station 391559081341201, Ohio River at river mile 184.6, June to October, 1995, Continued.

Ift $=$ feet $; \mu \mathrm{s} / \mathrm{cm}=$ microsiemens per centimeter $;{ }^{\circ} \mathrm{C}=$ degrees Celsius;

$\mathrm{mg} / \mathrm{L}=$ milligrams per liter; . = data not collected

\begin{tabular}{|c|c|c|c|c|c|c|c|c|c|}
\hline Date & Time & $\begin{array}{l}\text { Sampling } \\
\text { depth } \\
\text { (ft) }\end{array}$ & $\begin{array}{c}\text { Sample } \\
\text { location } \\
\text { (ft from } \\
\text { left bank) }\end{array}$ & $\begin{array}{l}\text { Specific } \\
\text { conduct- } \\
\text { ance } \\
(\mu S / \mathrm{cm})\end{array}$ & $\begin{array}{l}\text { pH } \\
\text { (stan- } \\
\text { dard } \\
\text { unlts) }\end{array}$ & $\begin{array}{c}\text { Temper- } \\
\text { ature, } \\
\text { water } \\
\left({ }^{\circ} \mathrm{C}\right)\end{array}$ & $\begin{array}{l}\text { Trans - } \\
\text { parency } \\
\text { (secchl } \\
\text { dlsk) } \\
\text { (ft) }\end{array}$ & $\begin{array}{l}\text { Dissolved } \\
\text { oxygen } \\
(\mathrm{mg} / \mathrm{L})\end{array}$ & $\begin{array}{c}\text { Dissolved } \\
\text { oxygen } \\
\text { (percent } \\
\text { satura- } \\
\text { tion) }\end{array}$ \\
\hline
\end{tabular}

\begin{tabular}{|c|c|c|c|c|c|c|c|c|c|}
\hline \multicolumn{10}{|c|}{ September } \\
\hline 20 & 0557 & 0.9 & 900 & 675 & 7.5 & 23.9 & $\cdots$ & 7.0 & 85 \\
\hline 20 & 0557 & 3.2 & 900 & 673 & 7.5 & 23.9 & $\cdots$ & 6.4 & 77 \\
\hline 20 & 0558 & 5.3 & 900 & 673 & 7.5 & 23.9 & $\cdots$ & 6.3 & 76 \\
\hline 20 & 0559 & 10 & 900 & 672 & 7.5 & 23.9 & $\cdots$ & 6.4 & 77 \\
\hline 20 & 0600 & 15 & 900 & 672 & 7.5 & 23.9 &.- & 6.2 & 76 \\
\hline 20 & 0601 & 20 & 900 & 675 & 7.5 & 23.9 & $\cdots$ & 6.2 & 76 \\
\hline 20 & 0602 & 23 & 900 & 674 & 7.5 & 23.9 & $-\cdot$ & 6.3 & 76 \\
\hline 20 & 1249 & 0.7 & 300 & 680 & 7.6 & 23.8 & - & 7.1 & \\
\hline 20 & 1249 & 2.9 & 300 & 681 & 7.5 & 23.9 & $\cdots$ & 7.3 & \\
\hline 20 & 1250 & 5.0 & 300 & 680 & 7.5 & 23.9 & - - & 6.3 & \\
\hline 20 & 1250 & 9.7 & 300 & 678 & 7.5 & 23.9 & -- & 6.4 & 7 \\
\hline 20 & 1251 & 15 & 300 & 681 & 7.5 & 23.9 & $\cdots$ & 6.4 & \\
\hline 20 & 1251 & 20 & 300 & 682 & 7.5 & 23.9 & $\cdots$ & 6.3 & 75 \\
\hline 20 & 1252 & 25 & 300 & 679 & 7.5 & 23.9 & -- & 6.4 & 77 \\
\hline 20 & 1252 & 30 & 300 & 679 & 7.5 & 23.9 & $\cdots$ & 6.4 & 77 \\
\hline 20 & 1253 & 34 & 300 & 678 & 7.5 & 23.9 & $\cdots$ & 6.3 & 75 \\
\hline 20 & 1253 & 36 & 300 & 680 & 7.5 & 23.9 & - & 6.3 & 76 \\
\hline 20 & 1255 & 1.2 & 600 & 678 & 7.5 & 23.9 & $\cdots$ & 6.4 & 77 \\
\hline 20 & 1255 & 2.9 & 600 & 680 & 7.5 & 23.9 & $\cdots$ & 6.3 & \\
\hline 20 & 1256 & 5.1 & 600 & 678 & 7.5 & 23.9 & $\cdots$ & 6.3 & \\
\hline 20 & 1256 & 10 & 600 & 681 & 7.5 & 23.9 & $\cdots$ & 6.4 & 76 \\
\hline 20 & 1257 & 15 & 600 & 683 & 7.5 & 23.9 & $\cdots$ & 6.3 & 76 \\
\hline 20 & 1257 & 20 & 600 & 678 & 7.5 & 23.9 & $\cdots$ & 6.4 & 77 \\
\hline 20 & 1258 & 25 & 600 & 679 & 7.5 & 23.9 & $\cdots$ & 6.4 & 77 \\
\hline 20 & 1258 & 26 & 600 & 683 & 7.5 & 23.9 & $\cdots$ & 6.3 & 75 \\
\hline 20 & 1304 & 0.7 & 900 & 680 & 7.5 & 23.9 & $\cdots$ & 6.4 & 76 \\
\hline 20 & 1303 & 2.9 & 900 & 681 & 7.5 & 23.9 & $\cdots$ & 6.3 & \\
\hline 20 & 1303 & 5.0 & 900 & 679 & 7.5 & 23.9 & $\cdots$ & 6.3 & \\
\hline 20 & 1302 & 10 & 900 & 680 & 7.5 & 23.9 & $\cdots$ & 6.3 & \\
\hline 20 & 1301 & 14 & 900 & 681 & 7.5 & 23.9 & $\cdots$ & 6.3 & 76 \\
\hline 20 & 1301 & 20 & 900 & 680 & 7.5 & 23.9 & $\cdots$ & 6.3 & 76 \\
\hline 20 & 1300 & 25 & 900 & 680 & 7.5 & 23.9 & $\cdots$ & 6.3 & \\
\hline
\end{tabular}


Table 18. Water-quality data for station 391559081341201, Ohio River at river mile 184.6, June to October, 1995, Continued.

$\left[\mathrm{ft}=\right.$ feet $; \mu \mathrm{S} / \mathrm{cm}=$ microslemens per centimeter $;{ }^{\circ} \mathrm{C}=$ degrees Celsius;

$\mathrm{mg} / \mathrm{L}=\mathrm{milligrams}$ per liter: $\cdots$ = data not collected]

\begin{tabular}{|c|c|c|c|c|c|c|c|c|c|}
\hline Date & Time & $\begin{array}{l}\text { Sampling } \\
\text { depth } \\
\text { (ft) }\end{array}$ & $\begin{array}{c}\text { Sample } \\
\text { location } \\
\text { (ft from } \\
\text { left bank) }\end{array}$ & $\begin{array}{l}\text { Specific } \\
\text { conduct - } \\
\text { ance } \\
(\mu \mathrm{s} / \mathrm{cm})\end{array}$ & $\begin{array}{l}\text { pH } \\
\text { (stan- } \\
\text { dard } \\
\text { units) }\end{array}$ & $\begin{array}{c}\text { Temper- } \\
\text { ature, } \\
\text { water } \\
\left({ }^{\circ} \mathrm{C}\right)\end{array}$ & $\begin{array}{l}\text { Trans - } \\
\text { parency } \\
\text { (Secchi } \\
\text { disk) } \\
\text { (Et) }\end{array}$ & $\begin{array}{l}\text { Dissolved } \\
\text { oxygen } \\
(\mathrm{mg} / \mathrm{L})\end{array}$ & $\begin{array}{c}\text { Dissolved } \\
\text { oxygen } \\
\text { (percent } \\
\text { satura. } \\
\text { tion) }\end{array}$ \\
\hline
\end{tabular}

\begin{tabular}{|c|c|c|c|c|c|c|c|c|c|}
\hline \multicolumn{10}{|c|}{ October } \\
\hline 05 & 0726 & 1.0 & 300 & 633 & 7.4 & 21.7 & $\cdots$ & 6.7 & 78 \\
\hline 05 & 0727 & 2.8 & 300 & 641 & 7.4 & 21.7 & - & 6.8 & 78 \\
\hline 05 & 0727 & 5.1 & 300 & 644 & 7.3 & 21.8 & $\cdots$ & 6.8 & 79 \\
\hline 05 & 0728 & 9.8 & 300 & 628 & 7.3 & 21.7 & - & 6.6 & 76 \\
\hline 05 & 0729 & 15 & 300 & 617 & 7.3 & 21.7 & - & 6.6 & 76 \\
\hline 05 & 0730 & 20 & 300 & 619 & 7.3 & 21.7 & $\cdots$ & 6.5 & 75 \\
\hline 05 & 0731 & 24 & 300 & 616 & 7.3 & 21.7 & $\cdots$ & 6.5 & 75 \\
\hline 05 & 0733 & 29 & 300 & 611 & 7.3 & 21.6 & - & 6.4 & 74 \\
\hline 05 & 0732 & 32 & 300 & 591 & 7.3 & 21.6 & $\cdots$ & 6.2 & 71 \\
\hline 05 & 0736 & -0.4 & 600 & 642 & 7.3 & 21.7 & $\cdots$ & 6.8 & 79 \\
\hline 05 & 0736 & 2.9 & 600 & 648 & 7.3 & 21.8 & $\cdots$ & 6.9 & 80 \\
\hline 05 & 0737 & 4.5 & 600 & 645 & 7.3 & 21.8 & $\cdots$ & 6.9 & 80 \\
\hline 05 & 0738 & 9.8 & 600 & 647 & 7.3 & 21.8 & $\cdots$ & 6.9 & 80 \\
\hline 05 & 0738 & 15 & 600 & 648 & 7.3 & 21.8 & $\cdots$ & 6.8 & 75 \\
\hline 05 & 0739 & 19 & 600 & 606 & 7.3 & 21.5 & - & 6.6 & 76 \\
\hline 05 & 0740 & 25 & 600 & 580 & 7.2 & 21.5 & $\cdots$ & 6.2 & \\
\hline 05 & 0740 & 30 & 600 & 587 & 7.2 & 21.5 & $\cdots$ & 6.2 & 12 \\
\hline 05 & 0741 & 34 & 600 & 581 & 7.2 & 21.5 & $\cdots$ & 6.2 & \\
\hline 05 & 0748 & 0.5 & 900 & 643 & 7.4 & 21.8 & $\cdots$ & 7.1 & 82 \\
\hline 05 & 0749 & 3.0 & 900 & 646 & 7.3 & 21.8 & $\cdots$ & 7.0 & 81 \\
\hline 05 & 0750 & 4.8 & 900 & 648 & 7.3 & 21.8 & $\cdots$ & 6.9 & 80 \\
\hline 05 & 0750 & 9.6 & 900 & 651 & 7.3 & 21.8 & $\cdots$ & 6.8 & 79 \\
\hline 05 & 0751 & 15 & 900 & 655 & 7.3 & 21.8 & $\cdots$ & 6.8 & 78 \\
\hline 05 & 0752 & 20 & 900 & 643 & 7.3 & 21.7 & $\cdots$ & 6.7 & 7 \\
\hline 05 & 0753 & 25 & 900 & 619 & 7.1 & 21.2 & $\cdots$ & 5.4 & \\
\hline 05 & 0754 & 30 & 900 & 431 & 7.1 & 21.1 & $\cdots$ & 5.2 & 60 \\
\hline 05 & 1451 & 0.2 & 300 & 624 & 7.5 & 21.8 & $\cdots$ & 6.8 & 78 \\
\hline 05 & 1451 & 3.1 & 300 & 625 & 7.5 & 21.8 & $\cdots$ & 6.7 & 78 \\
\hline 05 & 1452 & 5.1 & 300 & 628 & 7.5 & 21.8 & $\cdots$ & 6.7 & 77 \\
\hline 05 & 1453 & 9.9 & 300 & 516 & 7.4 & 21.3 & $\cdots$ & 6.7 & 7 \\
\hline 05 & 1453 & 15 & 300 & 607 & 7.4 & 21.7 & $\cdots$ & 6.2 & 7 \\
\hline 05 & 1454 & 20 & 300 & 475 & 7.3 & 21.1 & $\cdots$ & 5.7 & 6 \\
\hline 05 & 1454 & 25 & 300 & 477 & 7.2 & 21.1 & $\cdots$ & 5.4 & 6 \\
\hline 05 & 1455 & 30 & 300 & 395 & 7.2 & 20.8 & $\cdots$ & 5.3 & 6 \\
\hline 05 & 1456 & 35 & 300 & 404 & 7.2 & 20.8 & $\cdots$ & 5.0 & \\
\hline
\end{tabular}


Table 18. Water-quality data for station 391559081341201, Ohio River at river mile 184.6, June to October, 1995, Continued.

[ft $=$ feet; $\mu \mathrm{S} / \mathrm{cm}=$ microsıemens per centimeter; ${ }^{\circ} \mathrm{C}=$ degrees Celsius;

$\mathrm{mg} / \mathrm{L}=$ milligrams per liter; $\cdots$ = data not collected]

\begin{tabular}{|c|c|c|c|c|c|c|c|c|c|}
\hline Date & Time & $\begin{array}{l}\text { Sampling } \\
\text { depth } \\
\text { (ft) }\end{array}$ & $\begin{array}{c}\text { Sample } \\
\text { location } \\
\text { (tt trom } \\
\text { left bank) }\end{array}$ & $\begin{array}{l}\text { Specific } \\
\text { conduct- } \\
\text { ance } \\
(\mu \mathrm{S} / \mathrm{cm})\end{array}$ & $\begin{array}{l}\text { pH } \\
\text { (stan- } \\
\text { dard } \\
\text { units) }\end{array}$ & $\begin{array}{l}\text { Temper- } \\
\text { ature, } \\
\text { water } \\
\left({ }^{\circ} \mathrm{C}\right)\end{array}$ & $\begin{array}{l}\text { Trans - } \\
\text { parency } \\
\text { (Secchl } \\
\text { disk) } \\
\text { (Et) }\end{array}$ & $\begin{array}{c}\text { Dissolved } \\
\text { oxygen } \\
(\mathrm{mg} / \mathrm{L})\end{array}$ & $\begin{array}{c}\text { Dissolved } \\
\text { oxygen } \\
\text { (percent } \\
\text { satura. } \\
\text { tion) }\end{array}$ \\
\hline
\end{tabular}

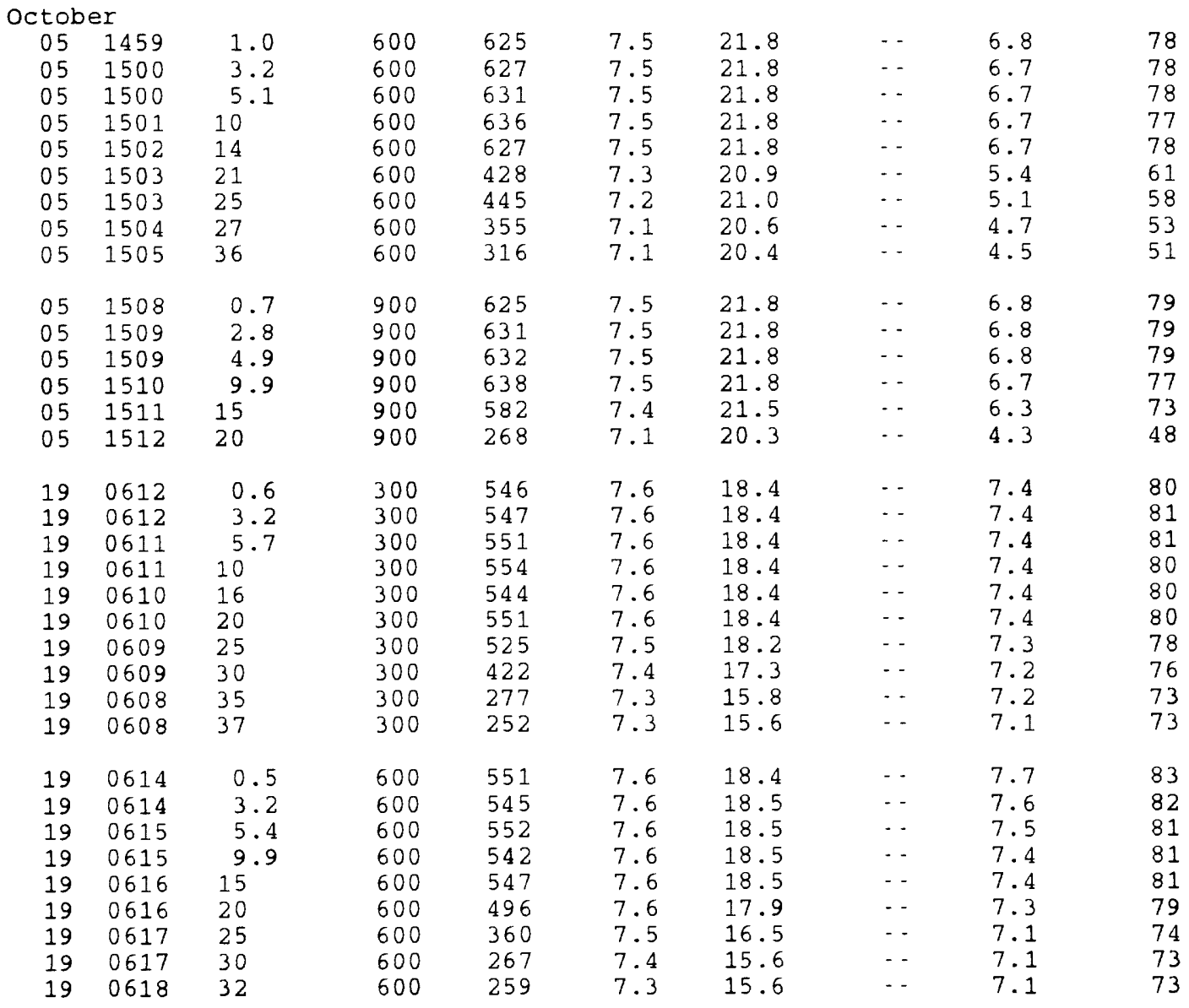


Table 18. Water-quality data for station 391559081341201, Ohio River at river mile 184.6, June to October, 1995, Continued.

$\left[\mathrm{ft}=\right.$ feet $; \mu \mathrm{s} / \mathrm{cm}=\mathrm{m}$ crosiemens per centimeter $;{ }^{\circ} \mathrm{C}=$ degrees Celsius;

$\mathrm{mg} / \mathrm{L}=\mathrm{milligrams}$ per liter; .. = data not collected]

\begin{tabular}{|c|c|c|c|c|c|c|c|c|c|}
\hline Date & Time & $\begin{array}{l}\text { Sampling } \\
\text { depth } \\
(\mathrm{f} t)\end{array}$ & $\begin{array}{l}\text { Sample } \\
\text { location } \\
\text { (tt from } \\
\text { lett bank) }\end{array}$ & $\begin{array}{c}\text { Specific } \\
\text { conduct- } \\
\text { ance } \\
(\mu \mathrm{S} / \mathrm{cm})\end{array}$ & $\begin{array}{l}\mathrm{pH} \\
\text { (stan- } \\
\text { dard } \\
\text { units) }\end{array}$ & $\begin{array}{c}\text { Temper- } \\
\text { ature, } \\
\text { water } \\
\left({ }^{\circ} \mathrm{C}\right)\end{array}$ & $\begin{array}{l}\text { Trans. } \\
\text { parency } \\
\text { (Secchi } \\
\text { disk) } \\
\text { (ft) }\end{array}$ & $\begin{array}{l}\text { Dissolved } \\
\text { oxygen } \\
(\mathrm{mg} / \mathrm{L})\end{array}$ & $\begin{array}{c}\text { Dissolved } \\
\text { oxygen } \\
\text { (percent } \\
\text { satura- } \\
\text { tron) }\end{array}$ \\
\hline \multicolumn{10}{|c|}{ October } \\
\hline 19 & 0623 & 0.8 & 900 & 547 & 7.6 & 18.4 & -- & 7.4 & 80 \\
\hline 19 & 0622 & 3.3 & 900 & 545 & 7.6 & 18.4 & $\ldots$ & 7.4 & 80 \\
\hline 19 & 0622 & 5.1 & 900 & 545 & 7.6 & 18.4 & $\ldots$ & 7.4 & 80 \\
\hline 19 & 0621 & 10 & 900 & 549 & 7.6 & 18.4 & - & 7.3 & 79 \\
\hline 19 & 0621 & 15 & 900 & 540 & 7.5 & 18.3 & $\cdots$ & 7.3 & 79 \\
\hline 19 & 0620 & 21 & 900 & 398 & 7.5 & 16.9 & - & 7.3 & 77 \\
\hline 19 & 0620 & 23 & 900 & 374 & 7.4 & 16.7 & - & 7.2 & 75 \\
\hline 19 & 1433 & 1.1 & 300 & 552 & 7.5 & 18.8 & $\cdots$ & 7.8 & 85 \\
\hline 19 & 1433 & 3.1 & 300 & 550 & 7.5 & 18.9 & $\cdots$ & 7.8 & 85 \\
\hline 19 & 1434 & 5.0 & 300 & 553 & 7.5 & 18.8 & $\cdots$ & 7.8 & 85 \\
\hline 19 & 1434 & 9.9 & 300 & 553 & 7.5 & 18.7 & $\cdots$ & 7.7 & 83 \\
\hline 19 & 1435 & 15 & 300 & 554 & 7.5 & 18.7 & $\cdots$ & 7.7 & 83 \\
\hline 19 & 1435 & 20 & 300 & 547 & 7.5 & 18.7 & -- & 7.7 & 83 \\
\hline 19 & 1436 & 25 & 300 & 526 & 7.5 & 18.5 & - & 7.7 & 83 \\
\hline 19 & 1436 & 30 & 300 & 503 & 7.5 & 18.1 & $\cdots$ & 7.7 & 82 \\
\hline 19 & 1437 & 35 & 300 & 337 & 7.3 & 16.1 & $\cdots$ & 7.4 & 76 \\
\hline 19 & 1437 & 37 & 300 & 305 & 7.3 & 15.9 & $\cdots$ & 7.4 & 76 \\
\hline 19 & 1439 & 0.4 & 600 & 552 & 7.5 & 19.3 & $\cdots$ & 7.9 & 86 \\
\hline 19 & 1439 & 3.1 & 600 & 552 & 7.5 & 18.7 & 2.5 & 7.7 & 83 \\
\hline 19 & 1440 & 5.2 & 600 & 550 & 7.5 & 18.7 & - & 7.7 & 83 \\
\hline 19 & 1440 & 9.9 & 600 & 551 & 7.5 & 18.7 & $\cdots$ & 7.7 & 83 \\
\hline 19 & 1441 & 15 & 600 & 530 & 7.5 & 18.4 & $\cdots$ & 7.6 & 81 \\
\hline 19 & 1441 & 20 & 600 & 508 & 7.5 & 18.2 & - & 7.6 & 81 \\
\hline 19 & 1442 & 25 & 600 & 444 & 7.4 & 17.3 & $\cdots$ & 7.3 & 77 \\
\hline 19 & 1442 & 30 & 600 & 340 & 7.3 & 16.3 & $\cdots$ & 7.4 & 76 \\
\hline 19 & 1444 & 0.6 & 900 & 553 & 7.5 & 19.3 & $\cdots$ & 8.0 & 88 \\
\hline 19 & 1444 & 3.1 & 900 & 552 & 7.5 & 18.8 & $\cdots$ & 7.9 & 86 \\
\hline 19 & 1445 & 5.2 & 900 & 552 & 7.5 & 18.7 & - & 7.8 & 84 \\
\hline 19 & 1445 & 9.9 & 900 & 552 & 7.5 & 18.7 & -- & 7.7 & 83 \\
\hline 19 & 1446 & 15 & 900 & 552 & 7.5 & 18.7 & $\cdots$ & 7.6 & 83 \\
\hline 19 & 1446 & 20 & 900 & 509 & 7.5 & 18.3 & $\cdots$ & 7.6 & 82 \\
\hline 19 & 1447 & 22 & 900 & 453 & 7.4 & 17.6 & $\cdots$ & 7.3 & 77 \\
\hline
\end{tabular}


Table 19. Water-quality data for station 391628081360401 , Ohio River at river mile 186.5, main channel, June to October, 1995.

$\left[E t=\right.$ feet; $\mu \mathrm{S} / \mathrm{cm}=$ microsiemens per centimeter $;{ }^{\circ} \mathrm{C}=$ degrees Celsius: $\mathrm{mg} / \mathrm{L}=\mathrm{m}$ lligrams per liter; - = data not collected]

\begin{tabular}{|c|c|c|c|c|c|c|c|c|c|}
\hline Date & Time & $\begin{array}{l}\text { Sampling } \\
\text { depth } \\
\text { (ft) }\end{array}$ & $\begin{array}{l}\text { Sample } \\
\text { location } \\
\text { (tt from } \\
\text { left bank) }\end{array}$ & $\begin{array}{c}\text { Specific } \\
\text { conduct - } \\
\text { ance } \\
(\mu \mathrm{S} / \mathrm{cm})\end{array}$ & $\begin{array}{l}\mathrm{pH} \\
\text { (stan- } \\
\text { dard } \\
\text { units) }\end{array}$ & $\begin{array}{c}\text { Temper- } \\
\text { ature, } \\
\text { water } \\
\left({ }^{\circ} \mathrm{C}\right)\end{array}$ & $\begin{array}{l}\text { Trans- } \\
\text { parency } \\
\text { (Secchl } \\
\text { disk) } \\
\text { (ft) }\end{array}$ & $\begin{array}{l}\text { Dissolved } \\
\text { oxygen } \\
(\mathrm{mg} / \mathrm{L})\end{array}$ & $\begin{array}{c}\text { Dissolved } \\
\text { oxygen } \\
\text { (percent } \\
\text { satura- } \\
\text { tion) }\end{array}$ \\
\hline
\end{tabular}

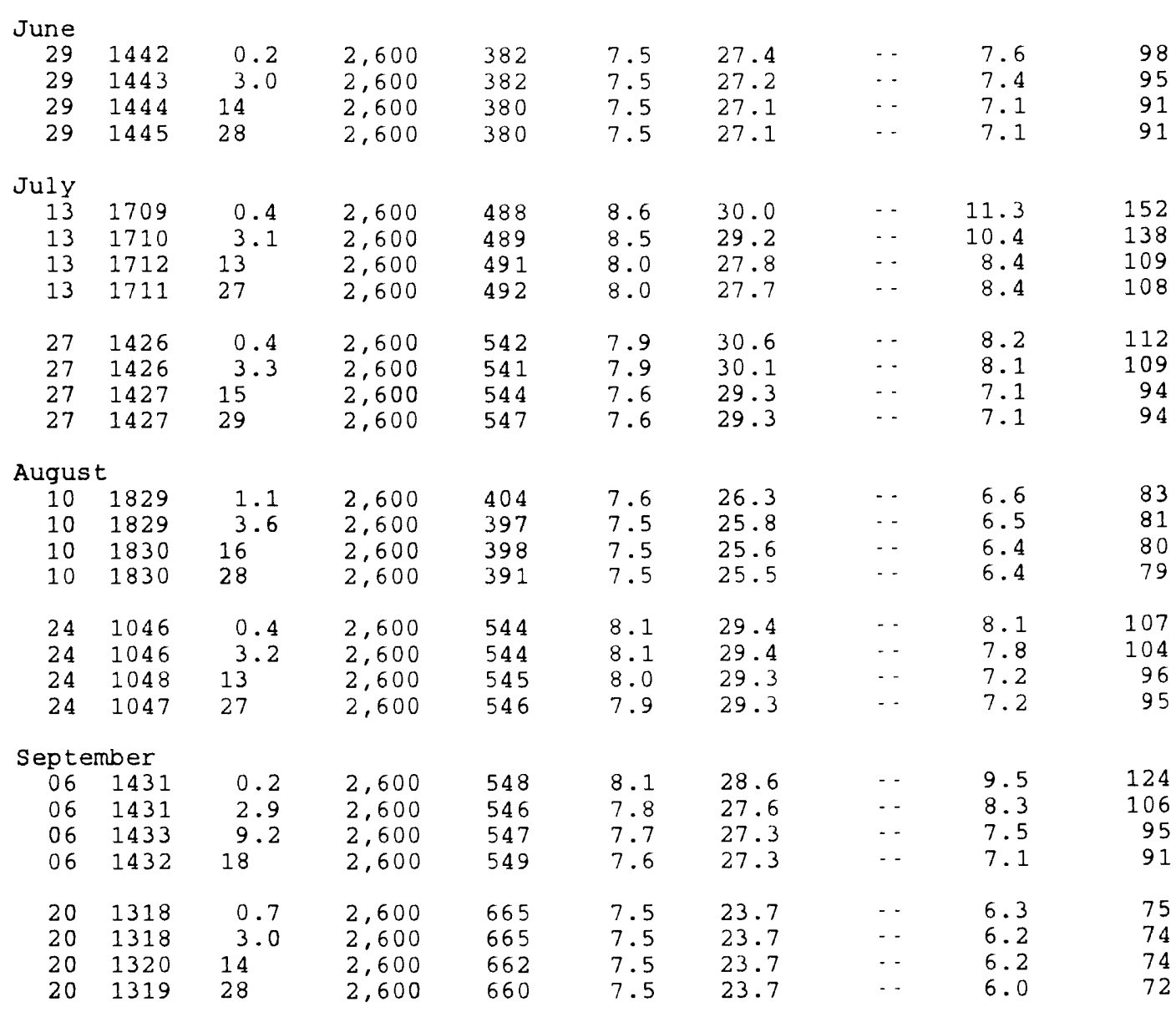


Table 19. Water-quality data for station 391628081360401, Ohio River at river mile 186.5, main channel, June to October, 1995, Continued.

$\left[\mathrm{ft}=\mathrm{feet} ; \mu \mathrm{S} / \mathrm{cm}=\right.$ microsiemens per centımeter $;{ }^{\circ} \mathrm{C}=$ degrees celsius; $\mathrm{mg} / \mathrm{L}=\mathrm{milligrams}$ per liter; $\cdots$ = data not collected]

\begin{tabular}{|c|c|c|c|c|c|c|c|c|c|}
\hline Date & Time & $\begin{array}{l}\text { Sampling } \\
\text { depth } \\
\text { (ft) }\end{array}$ & $\begin{array}{c}\text { Sample } \\
\text { location } \\
\text { (Et from } \\
\text { left bank) }\end{array}$ & $\begin{array}{l}\text { Specitic } \\
\text { conduct- } \\
\text { ance } \\
(\mu \mathrm{S} / \mathrm{cm})\end{array}$ & $\begin{array}{l}\mathrm{pH} \\
\text { (stan- } \\
\text { dard } \\
\text { units) }\end{array}$ & $\begin{array}{c}\text { Temper- } \\
\text { ature, } \\
\text { water } \\
\left({ }^{\circ} \mathrm{C}\right)\end{array}$ & $\begin{array}{l}\text { Trans- } \\
\text { parency } \\
\text { (Secchi } \\
\text { disk) } \\
\text { (ft) }\end{array}$ & $\begin{array}{c}\text { Dissolved } \\
\text { oxygen } \\
(\mathrm{mg} / \mathrm{L})\end{array}$ & $\begin{array}{c}\text { Dissolved } \\
\text { oxygen } \\
\text { (percent } \\
\text { satura. } \\
\text { tron) }\end{array}$ \\
\hline \multicolumn{10}{|c|}{ October } \\
\hline 05 & 1104 & 0.3 & 2,600 & 599 & 7.3 & 21.6 & - & 6.8 & 79 \\
\hline 05 & 1104 & 3.1 & 2,600 & 604 & 7.3 & 21.6 & $\ldots$ & 6.8 & 78 \\
\hline 05 & 1106 & 13 & 2,600 & 613 & 7.3 & 21.6 & - & 6.7 & 77 \\
\hline 05 & 1105 & 26 & 2,600 & 616 & 7.3 & 21.7 & - & 6.7 & 77 \\
\hline 19 & 1455 & 0.3 & 2,600 & 535 & 7.5 & 18.7 & -- & 8.1 & 88 \\
\hline 19 & 1456 & 3.1 & 2,600 & 536 & 7.5 & 18.6 & - & 7.9 & 85 \\
\hline 19 & 1457 & 13 & 2,600 & 538 & 7.5 & 18.5 & $\ldots$ & 7.8 & 84 \\
\hline 19 & 1456 & 27 & 2,600 & 531 & 7.5 & 18.5 & $\cdots$ & 7.8 & 84 \\
\hline
\end{tabular}


Table 20. Water-quality data for station 391604081361301, Ohio River at river mile 186.5, back channel, June to October, 1995.

$\mathrm{ft}=\mathrm{feet} ; \mu \mathrm{S} / \mathrm{cm}=$ microsiemens per centimeter; ${ }^{\circ} \mathrm{C}=$ degrees Celsius; $\mathrm{mg} / \mathrm{L}=$ milligrams per liter; $\cdots$ = data not collected]

\begin{tabular}{|c|c|c|c|c|c|c|c|c|c|}
\hline Date & Time & $\begin{array}{l}\text { Sampling } \\
\text { depth } \\
\text { (It) }\end{array}$ & $\begin{array}{c}\text { Sample } \\
\text { location } \\
\text { (ft trom } \\
\text { left bank) }\end{array}$ & $\begin{array}{l}\text { Specific } \\
\text { conduct. } \\
\text { ance } \\
(\mu \mathrm{s} / \mathrm{cm})\end{array}$ & $\begin{array}{l}\mathrm{pH} \\
\text { (stan- } \\
\text { dard } \\
\text { undts) }\end{array}$ & $\begin{array}{c}\text { Temper- } \\
\text { ature, } \\
\text { water } \\
\left({ }^{\circ} \mathrm{C}\right)\end{array}$ & $\begin{array}{c}\text { Trans - } \\
\text { parency } \\
\text { (secchi } \\
\text { disk) } \\
(t t)\end{array}$ & $\begin{array}{l}\text { Dissolved } \\
\text { oxygen } \\
(\mathrm{mg} / \mathrm{L})\end{array}$ & $\begin{array}{c}\text { Dissolved } \\
\text { oxygen } \\
\text { (percent } \\
\text { satura- } \\
\text { tion) }\end{array}$ \\
\hline
\end{tabular}

\begin{tabular}{|c|c|c|c|c|c|c|c|c|c|}
\hline \multicolumn{10}{|l|}{ June } \\
\hline 29 & 1455 & 0.7 & 400 & 390 & 7.6 & 28.0 & $\cdots$ & 7.8 & 102 \\
\hline 29 & 1455 & 3.1 & 400 & 370 & 7.5 & 27.2 & - - & 7.6 & 98 \\
\hline 29 & 1456 & 10 & 400 & 370 & 7.4 & 27.1 & $\cdots$ & 7.1 & 91 \\
\hline 29 & 1457 & 20 & 400 & 364 & 7.4 & 27.0 & -- & 7.1 & 91 \\
\hline \multicolumn{10}{|l|}{ July } \\
\hline 13 & 1637 & 0.5 & 400 & 490 & 8.3 & 28.3 & $\cdots$ & 9.6 & 126 \\
\hline 13 & 1637 & 3.2 & 400 & 490 & 8.0 & 27.6 & $\cdots$ & 8.5 & 110 \\
\hline 13 & 1639 & 10 & 400 & 488 & 7.8 & 27.5 & $-\cdot$ & 7.8 & 101 \\
\hline 13 & 1638 & 21 & 400 & 486 & 7.8 & 27.5 & $-\cdot$ & 7.9 & 102 \\
\hline 27 & 1431 & 0.4 & 400 & 535 & 8.1 & 30.5 & $\cdots$ & 8.5 & 116 \\
\hline 27 & 1431 & 3.1 & 400 & 541 & 7.7 & 29.4 & - & 7.3 & 97 \\
\hline 27 & 1433 & 11 & 400 & 537 & 7.6 & 29.3 & $\cdots$ & 7.2 & 96 \\
\hline 27 & 1432 & 21 & 400 & 544 & 7.6 & 29.3 & $\cdots$ & 7.1 & 95 \\
\hline \multicolumn{10}{|c|}{ August } \\
\hline 10 & 1825 & 1.0 & 400 & 392 & 7.6 & 26.7 & $\cdots$ & 6.5 & 83 \\
\hline 10 & 1825 & 3.8 & 400 & 389 & 7.5 & 25.6 & $\cdots$ & 6.4 & 80 \\
\hline 10 & 1826 & 11 & 400 & 377 & 7.5 & 25.4 & $\cdots$ & 6.3 & 79 \\
\hline 10 & 1826 & 21 & 400 & 371 & 7.5 & 25.2 & $\cdots$ & 6.4 & 79 \\
\hline 24 & 1051 & 0.4 & 400 & 543 & 8.3 & 29.6 & $\cdots$ & 9.1 & 121 \\
\hline 24 & 1052 & 3.3 & 400 & 542 & 8.1 & 29.4 & $\cdots$ & 7.9 & 105 \\
\hline 24 & 1052 & 9.1 & 400 & 541 & 7.9 & 29.3 & $\cdots$ & 7.0 & 93 \\
\hline 24 & 1053 & 19 & 400 & 541 & 7.9 & 29.3 & $\cdots$ & -- & $\cdots$ \\
\hline \multicolumn{10}{|c|}{ september } \\
\hline 06 & 1436 & 0.2 & 400 & 547 & 8.2 & 28.8 & $\cdots$ & 9.7 & 127 \\
\hline 06 & 1437 & 2.8 & 400 & 544 & 7.9 & 27.6 & $\cdots$ & 8.6 & 110 \\
\hline 06 & 1438 & 13 & 400 & 545 & 7.6 & 27.3 & $\cdots$ & 7.2 & 92 \\
\hline 06 & 1437 & 19 & 400 & 546 & 7.6 & 27.3 & $-\cdot$ & 7.1 & 91 \\
\hline 20 & 1309 & 0.6 & 400 & 659 & 7.5 & 23.7 & $\cdots$ & 6.1 & 73 \\
\hline 20 & 1310 & 3.0 & 400 & 660 & 7.5 & 23.7 & $\cdots$ & 6.1 & 73 \\
\hline 20 & 1311 & 11 & 400 & 659 & 7.5 & 23.7 & $\cdots$ & 6.2 & 74 \\
\hline 20 & 1310 & 19 & 400 & 658 & 7.5 & 23.7 & $\cdots$ & 6.1 & 73 \\
\hline
\end{tabular}


Table 20. Water-quality data for station 391604081361301, Ohio River at river mile 186.5, back channel, June to October, 1995, Continued.

$\left[\mathrm{ft}=\mathrm{feet} ; \mu \mathrm{S} / \mathrm{cm}=\right.$ microsiemens per centimeter $;{ }^{\circ} \mathrm{C}=$ degrees Celsius; $\mathrm{mg} / \mathrm{L}=\mathrm{mili}$ igrams per liter; $\cdots$ = data not collected]

\begin{tabular}{|c|c|c|c|c|c|c|c|c|c|}
\hline Date & Time & $\begin{array}{l}\text { Sampling } \\
\text { depth } \\
\text { (ft) }\end{array}$ & $\begin{array}{c}\text { Sample } \\
\text { location } \\
\text { (tt trom } \\
\text { lett bank) }\end{array}$ & $\begin{array}{l}\text { Specific } \\
\text { conduct- } \\
\text { ance } \\
(\mu \mathrm{S} / \mathrm{cm})\end{array}$ & $\begin{array}{l}\mathrm{pH} \\
\text { (stan- } \\
\text { dard } \\
\text { units) }\end{array}$ & $\begin{array}{c}\text { Temper- } \\
\text { ature, } \\
\text { water } \\
\left({ }^{\circ} \mathrm{C}\right)\end{array}$ & $\begin{array}{l}\text { Trans- } \\
\text { parency } \\
\text { (Secch1 } \\
\text { disk) } \\
(t t)\end{array}$ & $\begin{array}{l}\text { Dissolved } \\
\text { oxygen } \\
\text { (mg/L) }\end{array}$ & $\begin{array}{c}\text { Dissolved } \\
\text { oxygen } \\
\text { (percent } \\
\text { satura- } \\
\text { tion) }\end{array}$ \\
\hline \multicolumn{10}{|c|}{ October } \\
\hline 05 & 1110 & 0.3 & 400 & 616 & 7.3 & 21.7 & $\cdots$ & 6.7 & 78 \\
\hline 05 & 1110 & 2.9 & 400 & 621 & 7.3 & 21.7 & $\cdots$ & 6.7 & 78 \\
\hline 05 & 1116 & 9.6 & 400 & 630 & 7.3 & 21.7 & $\cdots$ & 6.7 & 77 \\
\hline 05 & 1113 & 21 & 400 & 633 & 7.3 & 21.7 & $\cdots$ & 6.6 & 76 \\
\hline 19 & 1451 & 0.5 & 400 & 544 & 7.6 & 19.2 & - & 8.2 & 90 \\
\hline 19 & 1451 & 3.0 & 400 & 544 & 7.6 & 19.2 & $\cdots$ & 8.1 & 89 \\
\hline 19 & 1452 & 9.7 & 400 & 518 & 7.5 & 18.2 & $\cdots$ & 7.6 & 81 \\
\hline 19 & 1452 & 21 & 400 & 494 & 7.4 & 17.9 & $\cdots$ & 7.4 & 78 \\
\hline
\end{tabular}


Table 21. Water-quality data for station 391636081384701, Ohio River at river mile 189.0, main channel, June to October, 1995.

$\left[\mathrm{ft}=\mathrm{feet} ; \mu \mathrm{S} / \mathrm{cm}=\mathrm{microsiemens}\right.$ per centımeter $;{ }^{\circ} \mathrm{C}=$ degrees Celsius;

$\mathrm{mg} / \mathrm{L}=\mathrm{milligrams}$ per liter; .. = data not collected]

\begin{tabular}{|c|c|c|c|c|c|c|c|c|c|}
\hline Date & Time & $\begin{array}{l}\text { Sampling } \\
\text { depth } \\
\text { (ft) }\end{array}$ & $\begin{array}{c}\text { Sample } \\
\text { location } \\
\text { (ft from } \\
\text { left bank) }\end{array}$ & $\begin{array}{l}\text { Specific } \\
\text { conduct } \\
\text { ance } \\
(\mu \mathrm{s} / \mathrm{cm})\end{array}$ & $\begin{array}{l}\mathrm{pH} \\
\text { (stan- } \\
\text { dard } \\
\text { units) }\end{array}$ & $\begin{array}{c}\text { Temper- } \\
\text { ature, } \\
\text { water } \\
\left({ }^{\circ} \mathrm{C}\right)\end{array}$ & $\begin{array}{c}\text { Trans - } \\
\text { parency } \\
\text { (secchl } \\
\text { disk) } \\
\text { (tt) }\end{array}$ & $\begin{array}{c}\text { Dissolved } \\
\text { oxygen } \\
\text { (mg/L) }\end{array}$ & $\begin{array}{c}\text { Dissolved } \\
\text { oxygen } \\
\text { (percent } \\
\text { satura- } \\
\text { tion) }\end{array}$ \\
\hline
\end{tabular}

June

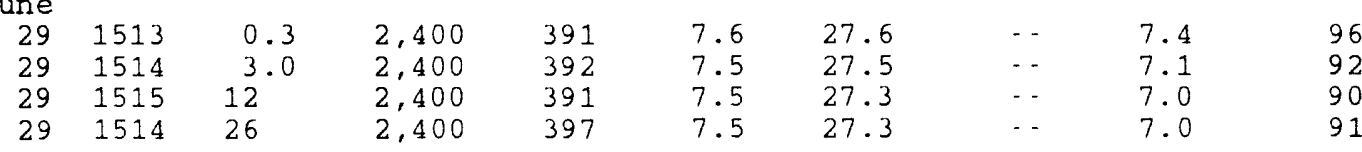

Ju1y

$\begin{array}{rcccccccrr}13 & 1723 & 0.6 & 2,400 & 481 & 8.7 & 30.3 & \ldots & 12.0 & 163 \\ 13 & 1723 & 2.9 & 2,400 & 480 & 8.5 & 28.9 & \ldots & 10.7 & 142 \\ 13 & 1724 & 13 & 2,400 & 480 & 7.9 & 27.7 & \ldots & 8.2 & 106 \\ 13 & 1724 & 26 & 2,400 & 480 & 7.9 & 27.5 & \ldots & 8.3 & 107 \\ 27 & 1445 & 0.4 & 2,400 & 538 & 8.2 & 29.9 & \ldots & 9.3 & 125 \\ 27 & 1445 & 3.3 & 2,400 & 541 & 7.8 & 29.3 & \ldots & 7.5 & 100 \\ 27 & 1446 & 13 & 2,400 & 542 & 7.6 & 29.1 & \ldots & 7.0 & 93 \\ 27 & 1446 & 26 & 2,400 & 542 & 7.6 & 29.1 & \ldots & 7.0 & 92\end{array}$

August

$\begin{array}{llcllllllr}10 & 1814 & 1.1 & 2,400 & 379 & 7.6 & 26.6 & \ldots & 6.7 & 85 \\ 10 & 1814 & 3.5 & 2,400 & 381 & 7.5 & 25.8 & \ldots & 6.5 & 82 \\ 10 & 1815 & 13 & 2,400 & 386 & 7.5 & 25.0 & \ldots & 6.4 & 78 \\ 10 & 1815 & 26 & 2,400 & 378 & 7.5 & 25.0 & \ldots & 6.3 & 78 \\ & & & & & & & & & 122 \\ 24 & 1039 & 0.5 & 2,400 & 554 & 8.4 & 30.0 & \ldots & 9.0 & 104 \\ 24 & 1039 & 3.5 & 2,400 & 548 & 8.1 & 29.5 & \ldots & 7.8 & 98 \\ 24 & 1040 & 13 & 2,400 & 548 & 8.1 & 29.4 & \ldots & 7.4 & 95 \\ 24 & 1040 & 25 & 2,400 & 547 & 8.0 & 29.4 & \ldots & 7.1 & \end{array}$

september

$\begin{array}{rccccccccr}06 & 1455 & 0.2 & 2,400 & 569 & 8.3 & 30.3 & \cdots & 9.6 & 129 \\ 06 & 1455 & 2.8 & 2,400 & 540 & 8.2 & 28.5 & \ldots & 9.7 & 127 \\ 06 & 1456 & 12 & 2,400 & 540 & 7.6 & 27.4 & \cdots & 7.1 & 90 \\ 06 & 1455 & 25 & 2,400 & 537 & 7.6 & 27.4 & \cdots & 6.9 & 88 \\ & & & & & & & & & \\ 20 & 1343 & 0.9 & 2,400 & 656 & 7.6 & 23.7 & \ldots & 6.3 & 75 \\ 20 & 1343 & 2.9 & 2,400 & 656 & 7.5 & 23.7 & \cdots & 6.2 & 75 \\ 20 & 1345 & 13 & 2,400 & 655 & 7.5 & 23.7 & \ldots & 6.2 & 74 \\ 20 & 1344 & 26 & 2,400 & 656 & 7.5 & 23.7 & \cdots & 6.1 & 73\end{array}$


Table 21. Water-quality data for station 391636081384701, Ohio River at river mile 189.0, main channel, June to October, 1995, Continued.

[ft $=$ feet; $\mu \mathrm{S} / \mathrm{cm}=$ microsiemens per centımeter; ${ }^{\circ} \mathrm{C}=$ degrees Celsius;

$\mathrm{mg} / \mathrm{L}=\mathrm{mlli}$ igrams per liter; . = data not collected]

\begin{tabular}{|c|c|c|c|c|c|c|c|c|c|}
\hline Date & Time & $\begin{array}{l}\text { Sampling } \\
\text { depth } \\
\text { (ft) }\end{array}$ & $\begin{array}{l}\text { Sample } \\
\text { location } \\
\text { (Et from } \\
\text { left bank) }\end{array}$ & $\begin{array}{l}\text { Specific } \\
\text { conduct. } \\
\text { ance } \\
(\mu \mathrm{S} / \mathrm{cm})\end{array}$ & $\begin{array}{l}\mathrm{pH} \\
\text { (stan- } \\
\text { dard } \\
\text { units) }\end{array}$ & $\begin{array}{c}\text { Temper- } \\
\text { ature. } \\
\text { water } \\
\left({ }^{\circ} \mathrm{C}\right)\end{array}$ & $\begin{array}{c}\text { Trans - } \\
\text { parency } \\
\text { (Secchi } \\
\text { dlsk) } \\
(\mathrm{Et})\end{array}$ & $\begin{array}{l}\text { Dissolved } \\
\text { oxygen } \\
\text { (mg/L) }\end{array}$ & $\begin{array}{c}\text { Dissolved } \\
\text { oxygen } \\
\text { (percent } \\
\text { satura- } \\
\text { tion) }\end{array}$ \\
\hline \multicolumn{10}{|c|}{ October } \\
\hline 05 & 1135 & 0.5 & 2,400 & 629 & 7.3 & 21.7 & $\cdots$ & 6.8 & 79 \\
\hline 05 & 1134 & 2.8 & 2,400 & 630 & 7.3 & 21.7 & $\cdots$ & 6.8 & 79 \\
\hline 05 & 1134 & 12 & 2,400 & 642 & 7.3 & 21.7 & $\ldots$ & 6.7 & 78 \\
\hline 05 & 1133 & 24 & 2,400 & 643 & 7.3 & 21.7 & $\cdots$ & 6.7 & 77 \\
\hline 19 & 1504 & 0.9 & 2,400 & 536 & 7.6 & 19.4 & $\cdots$ & 7.8 & 86 \\
\hline 19 & 1504 & 3.2 & 2,400 & 536 & 7.6 & 19.2 & - & 7.8 & 85 \\
\hline 19 & 1506 & 13 & 2,400 & 537 & 7.5 & 18.6 & - & 7.6 & 82 \\
\hline 19 & 1505 & 25 & 2,400 & 536 & 7.5 & 18.4 & $\cdots$ & 7.6 & 82 \\
\hline
\end{tabular}


Table 22. Water-quality data for station 391616081385001, Ohio River at river mile 189.0, back channel, June to October, 1995.

$\left[\mathrm{ft}=\right.$ feet $; \mu \mathrm{s} / \mathrm{cm}=$ microsiemens per centimeter $;{ }^{\circ} \mathrm{C}=$ degrees Celsius;

$\mathrm{mg} / \mathrm{L}=$ milligrams per liter; - = data not collected]

\begin{tabular}{|c|c|c|c|c|c|c|c|c|c|}
\hline Date & Time & $\begin{array}{l}\text { Sampling } \\
\text { depth } \\
(E t)\end{array}$ & $\begin{array}{c}\text { Sample } \\
\text { location } \\
\text { (tt from } \\
\text { lett bank) }\end{array}$ & $\begin{array}{l}\text { Specitic } \\
\text { conduct. } \\
\text { ance } \\
(\mu \mathrm{s} / \mathrm{cm})\end{array}$ & $\begin{array}{l}\mathrm{pH} \\
\text { (stan. } \\
\text { dard } \\
\text { units) }\end{array}$ & $\begin{array}{c}\text { Temper- } \\
\text { ature, } \\
\text { water } \\
\left({ }^{\circ} \mathrm{C}\right)\end{array}$ & $\begin{array}{l}\text { Trans - } \\
\text { parency } \\
\text { (Secchl } \\
\text { disk) } \\
(\mathrm{ft})\end{array}$ & $\begin{array}{l}\text { Dissolved } \\
\text { oxygen } \\
(\mathrm{mg} / \mathrm{L})\end{array}$ & $\begin{array}{c}\text { Dissolved } \\
\text { oxygen } \\
\text { (percent } \\
\text { satura- } \\
\text { tion) }\end{array}$ \\
\hline
\end{tabular}

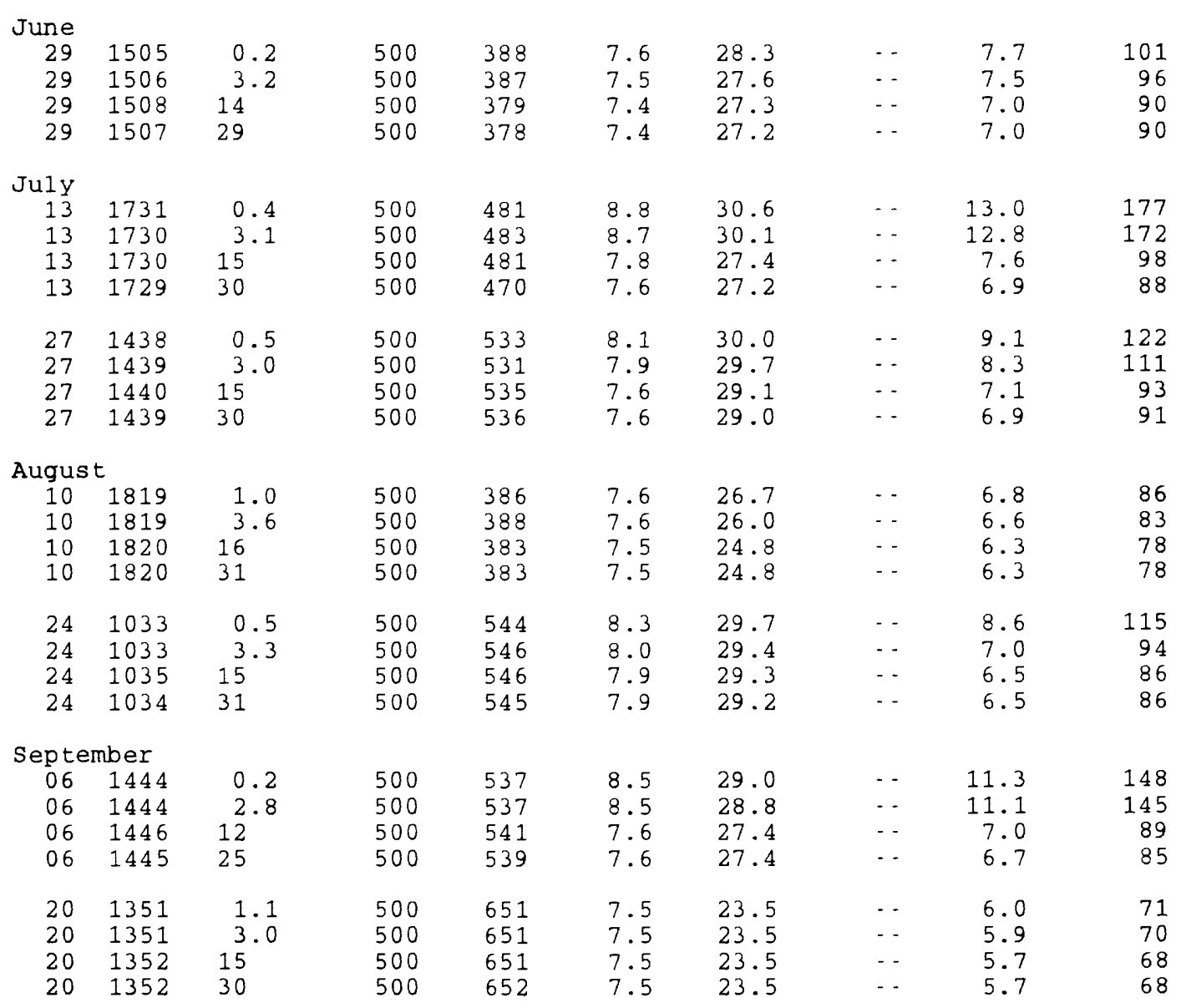


Table 22. Water-quality data for station 391616081385001, Ohio River at river mile 189.0, back channel, June to October, 1995, Continued.

[ft $=$ feet $; \mu \mathrm{S} / \mathrm{cm}=$ microsiemens per centimeter $;{ }^{\circ} \mathrm{C}=$ degrees Celsius;

$\mathrm{mg} / \mathrm{L}=\mathrm{milligrams}$ per liter; $-\cdot$ = data not collected]

\begin{tabular}{|c|c|c|c|c|c|c|c|c|c|}
\hline Date & Time & $\begin{array}{l}\text { Sampling } \\
\text { depth } \\
\text { (ft) }\end{array}$ & $\begin{array}{l}\text { Sample } \\
\text { location } \\
\text { (ft from } \\
\text { left bank) }\end{array}$ & $\begin{array}{l}\text { Specific } \\
\text { conduct- } \\
\text { ance } \\
(\mu \mathrm{S} / \mathrm{cm})\end{array}$ & $\begin{array}{l}\mathrm{pH} \\
\text { (stan- } \\
\text { dard } \\
\text { units) }\end{array}$ & $\begin{array}{c}\text { Temper- } \\
\text { ature, } \\
\text { water } \\
\left({ }^{\circ} \mathrm{C}\right)\end{array}$ & $\begin{array}{c}\text { Trans- } \\
\text { parency } \\
\text { (secch1 } \\
\text { disk) } \\
\text { (ft) }\end{array}$ & $\begin{array}{c}\text { Dissolved } \\
\text { oxygen } \\
\text { (mg/L) }\end{array}$ & $\begin{array}{c}\text { Dissolved } \\
\text { oxygen } \\
\text { (percent } \\
\text { satura- } \\
\text { tion) }\end{array}$ \\
\hline \multicolumn{10}{|c|}{ October } \\
\hline 05 & 1127 & 0.4 & 500 & 628 & 7.3 & 21.7 & $\cdots$ & 6.7 & 78 \\
\hline 05 & 1126 & 3.4 & 500 & 626 & 7.3 & 21.7 & $\cdots$ & 6.8 & 78 \\
\hline 05 & 1124 & 12 & 500 & 626 & 7.3 & 21.7 & $\cdots$ & 6.7 & 77 \\
\hline 05 & 1122 & 27 & 500 & 638 & 7.3 & 21.7 & $\cdots$ & 6.5 & 75 \\
\hline 19 & 1510 & 0.5 & 500 & 531 & 7.7 & 19.8 & $\cdots$ & 8.5 & 94 \\
\hline 19 & 1510 & 3.2 & 500 & 523 & 7.5 & 18.4 & - & 7.7 & 83 \\
\hline 19 & 1512 & 14 & 500 & 519 & 7.4 & 18.1 & $\cdots$ & 7.5 & 81 \\
\hline 19 & 1511 & 30 & 500 & 511 & 7.4 & 18.1 & $\cdots$ & 7.4 & 79 \\
\hline
\end{tabular}


Table 23. Water-quality data for station 391601081411101, Ohio River at river mile 191.3, June to October, 1995.

$\left[\mathrm{ft}=\mathrm{feet} ; \mu \mathrm{S} / \mathrm{cm}=\right.$ microsiemens per centimeter; ${ }^{\circ} \mathrm{C}=$ degrees Celsius; $\mathrm{mg} / \mathrm{L}=\mathrm{milligrams}$ per liter; - - data not collected

\begin{tabular}{|c|c|c|c|c|c|c|c|c|c|}
\hline Date & Time & $\begin{array}{l}\text { Sampling } \\
\text { depth } \\
\text { (ft) }\end{array}$ & $\begin{array}{l}\text { Sample } \\
\text { location } \\
\text { (ft Erom } \\
\text { left bank) }\end{array}$ & $\begin{array}{l}\text { Specific } \\
\text { conduct- } \\
\text { ance } \\
(\mu \mathrm{s} / \mathrm{cm})\end{array}$ & $\begin{array}{l}\mathrm{pH} \\
\text { (stan- } \\
\text { dard } \\
\text { units) }\end{array}$ & $\begin{array}{c}\text { Temper- } \\
\text { ature, } \\
\text { water } \\
\left({ }^{\circ} \mathrm{C}\right)\end{array}$ & $\begin{array}{l}\text { Trans- } \\
\text { parency } \\
\text { (secchl } \\
\text { disk) } \\
(t t)\end{array}$ & $\begin{array}{l}\text { Dissolved } \\
\text { oxygen } \\
(\mathrm{mg} / \mathrm{L})\end{array}$ & $\begin{array}{c}\text { Dassolved } \\
\text { oxygen } \\
\text { (percent } \\
\text { satura- } \\
\text { tion) }\end{array}$ \\
\hline
\end{tabular}

June

$\begin{array}{rrrr}29 & 1520 & 0.2 & 6 \\ 29 & 1520 & 3.1 & 6 \\ 29 & 1522 & 16 & 6 \\ 29 & 1521 & 34 & 6\end{array}$

600

$600 \quad 414$

7.

7.5

28.0

- 7.4

7.3

66.9

$600 \quad 418$

7.5

27.5

$\cdots$

6.9

96

27.5

8.829 .5

$8.7 \quad 29.2$

$7.8 \quad 27.6$

$\begin{array}{lll}\text {.. } & 12.6 & 168\end{array}$

$\begin{array}{rcc}\text { Ju1y } & & \\ 13 & 1737 & 0.5 \\ 13 & 1737 & 3.3 \\ 13 & 1739 & 16 \\ 13 & 1738 & 33\end{array}$

600

468

600

600

468

464

7.7

27.2

-. 12.3

-
$\ldots$

- 6.8

164

$\begin{array}{lll}27 & 1452 & 0.5\end{array}$

$27 \quad 1452$

600

525

$600 \quad 529$

8.3

30.5

$7.8 \quad 29.4$

524

7.6

29.1

600

521

7.6

28.9

- 9.8

. 7.8

. 6.9

133

$\begin{array}{lll}27 & 1453 & 17 \\ 27 & 1453 & 35\end{array}$

August

$\begin{array}{ll}10 & 1807 \\ 10 & 1807\end{array}$

101808

0.9
3.5
18

600

600

485

7.8

27.6

471

443

600

7.7

26.8

$7.6 \quad 25.6$

451

25.7

-

6.6

- 7.3

-
$-\quad 7.0$

. 6.7

. 6.6

94

$24 \quad 1026$

0.4

600

522

$600 \quad 525$

$600 \quad 521$

8.3

29.8

- 8.6

. 7.3

8.0

29.6

29.5

-

(

6.9

8.0

29.5

- 6.9

89

$24 \quad 1027$

33

518

\section{September}

061503

061503

$06 \quad 1505$

$06 \quad 1504$

0.2
2.9

600

541

600

538

544

$8.4 \quad 29.3$

- 10.4

28.5

- 10.2

. 7.1

7.7

27.5

$\cdots$

6.8

137

7.7

27.4

$\begin{array}{ll}- & 6.1 \\ \ldots & 6.1\end{array}$

7.5

23.8

600

666

666

7.5

23.8

7.5

23.8

600
600

7.5

23.8

-

-

6.1

6.0

88

-

74

73

73 
Table 23. Water-quality data for station 391601081411101, Ohio River at river mile 191.3, June to October, 1995, Continued.

$\left[E t=E e e t ; \mu \mathrm{S} / \mathrm{cm}=\right.$ microsiemens per centimeter; ${ }^{\circ} \mathrm{C}=$ degrees Celsius;

$\mathrm{mg} / \mathrm{L}=\mathrm{mili}$ igrams per liter; $\cdot-$ = data not collected]

\begin{tabular}{|c|c|c|c|c|c|c|c|c|c|}
\hline Date & Time & $\begin{array}{l}\text { Sampling } \\
\text { depth } \\
\text { (Et) }\end{array}$ & $\begin{array}{c}\text { Sample } \\
\text { location } \\
\text { (ft trom } \\
\text { left bank) }\end{array}$ & $\begin{array}{l}\text { Specific } \\
\text { conduct - } \\
\text { ance } \\
(\mu \mathrm{S} / \mathrm{cm})\end{array}$ & $\begin{array}{l}\mathrm{pH} \\
\text { (stan- } \\
\text { dard } \\
\text { units) }\end{array}$ & $\begin{array}{c}\text { Temper- } \\
\text { ature, } \\
\text { water } \\
\left({ }^{\circ} \mathrm{C}\right)\end{array}$ & $\begin{array}{l}\text { Trans- } \\
\text { parency } \\
\text { (Secchl } \\
\text { disk) } \\
\text { (ft) }\end{array}$ & $\begin{array}{l}\text { Dissolved } \\
\text { oxygen } \\
\text { (mg/L) }\end{array}$ & $\begin{array}{c}\text { Dissolved } \\
\text { oxygen } \\
\text { (percent } \\
\text { satura- } \\
\text { tion) }\end{array}$ \\
\hline \multicolumn{10}{|c|}{ October } \\
\hline 05 & 1144 & 0.3 & 600 & 638 & 7.4 & 22.0 & $\cdots$ & 6.9 & 81 \\
\hline 05 & 1145 & 3.4 & 600 & 640 & 7.4 & 22.0 & $\cdots$ & 6.9 & 80 \\
\hline 05 & 1152 & 15 & 600 & 635 & 7.4 & 22.0 & - - & 6.8 & 79 \\
\hline 05 & 1150 & 31 & 600 & 639 & 7.4 & 22.0 & -- & 6.7 & 77 \\
\hline 19 & 1518 & 0.6 & 600 & 542 & 7.5 & 18.7 & - & 7.8 & 84 \\
\hline 19 & 1518 & 3.0 & 500 & 544 & 7.5 & 18.8 & $\cdots$ & 7.8 & 84 \\
\hline 19 & 1519 & 14 & 600 & 546 & 7.5 & 18.8 & - & 7.8 & 85 \\
\hline 19 & 1519 & 27 & 600 & 541 & 7.5 & 18.5 & $\cdots$ & 7.6 & 82 \\
\hline
\end{tabular}


Table 24. Water-quality data for station 391447081414201, Ohio River at river mile 192.9, June to October, 1995.

$\left[\mathrm{ft}=\mathrm{feet} ; \mu \mathrm{S} / \mathrm{cm}=\right.$ microsiemens per centimeter; ${ }^{\circ} \mathrm{C}=$ degrees Celsius; $\mathrm{mg} / \mathrm{L}=\mathrm{milligrams}$ per liter; $\cdots$ = data not collected]

\begin{tabular}{|c|c|c|c|c|c|c|c|c|c|}
\hline Date & Time & $\begin{array}{l}\text { Sampling } \\
\text { depth } \\
\text { (ft) }\end{array}$ & $\begin{array}{c}\text { Sample } \\
\text { location } \\
\text { (ft from } \\
\text { left bank) }\end{array}$ & $\begin{array}{l}\text { Specific } \\
\text { conduct- } \\
\text { ance } \\
(\mu \mathrm{s} / \mathrm{cm})\end{array}$ & $\begin{array}{l}\mathrm{pH} \\
\text { (stan. } \\
\text { dard } \\
\text { units) }\end{array}$ & $\begin{array}{c}\text { Temper- } \\
\text { ature, } \\
\text { water } \\
\left({ }^{\circ} \mathrm{C}\right)\end{array}$ & $\begin{array}{c}\text { Trans- } \\
\text { parency } \\
\text { (Secchl } \\
\text { dlsk) } \\
(\mathrm{Et})\end{array}$ & $\begin{array}{l}\text { Dissolved } \\
\text { oxygen } \\
(\mathrm{mg} / \mathrm{L})\end{array}$ & $\begin{array}{c}\text { Dissolved } \\
\text { oxygen } \\
\text { (percent } \\
\text { satura- } \\
\text { tion) }\end{array}$ \\
\hline
\end{tabular}

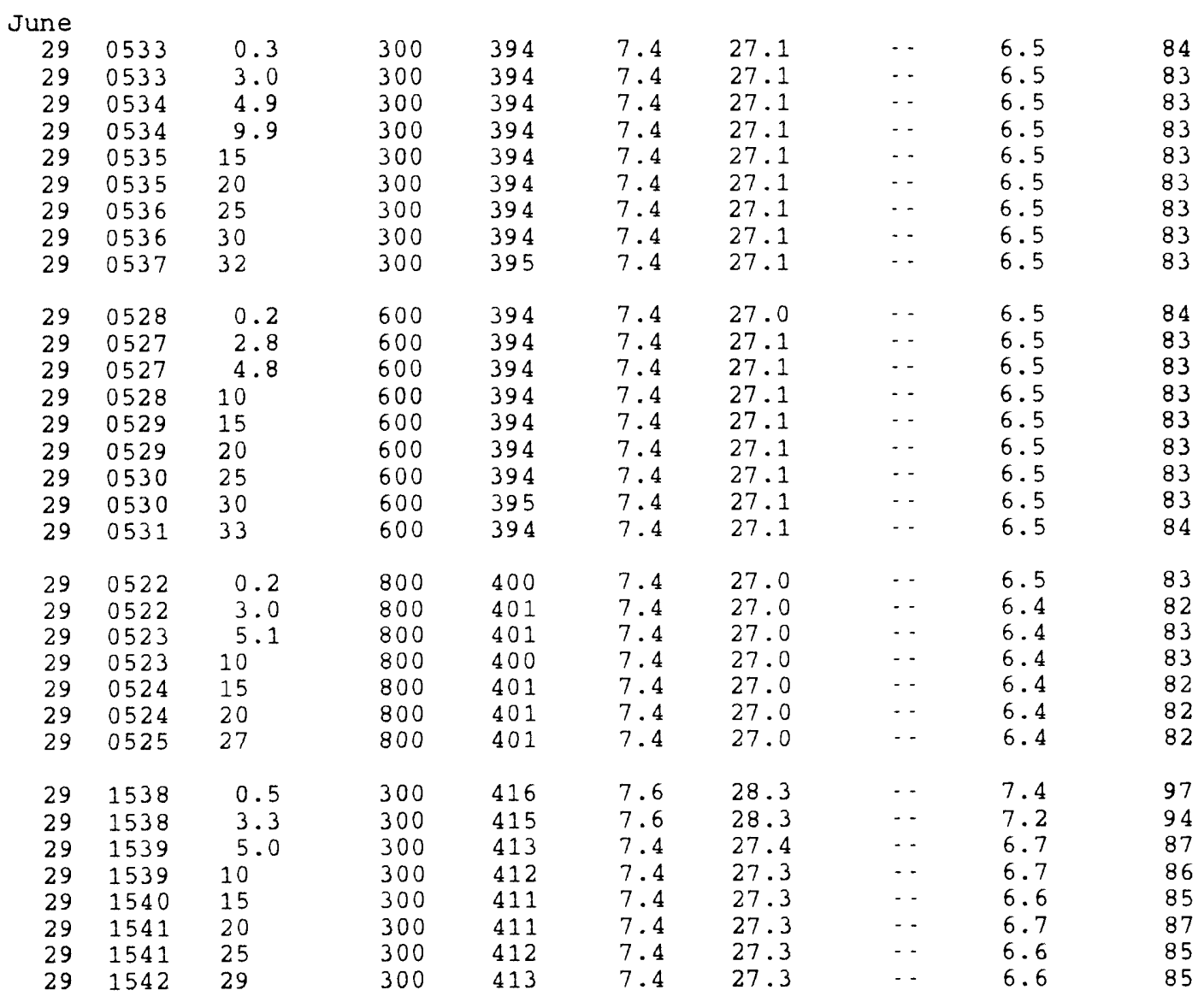


Table 24. Water-quality data for station 391447081414201, Ohio River at river mile 192.9, June to October, 1995, Continued.

lft $=$ feet; $\mu \mathrm{S} / \mathrm{cm}=$ microsiemens per centımeter; ${ }^{\circ} \mathrm{C}=$ degrees Celsius; $\mathrm{mg} / \mathrm{L}=$ miligrams per Iiter; $\cdots$ = data not collected]

\begin{tabular}{|c|c|c|c|c|c|c|c|c|c|}
\hline Date & Time & $\begin{array}{l}\text { Sampling } \\
\text { depth } \\
\text { (ft) }\end{array}$ & $\begin{array}{l}\text { Sample } \\
\text { location } \\
\text { (ft from } \\
\text { left bank) }\end{array}$ & $\begin{array}{l}\text { Specific } \\
\text { conduct- } \\
\text { ance } \\
(\mu \mathrm{S} / \mathrm{cm})\end{array}$ & $\begin{array}{l}\mathrm{pH} \\
\text { (stan- } \\
\text { dard } \\
\text { units) }\end{array}$ & $\begin{array}{c}\text { Temper- } \\
\text { ature, } \\
\text { water } \\
\left({ }^{\circ} \mathrm{C}\right)\end{array}$ & $\begin{array}{l}\text { Trans - } \\
\text { parency } \\
\text { (secchl } \\
\text { disk) } \\
\text { (ft) }\end{array}$ & $\begin{array}{l}\text { Dissolved } \\
\text { oxygen } \\
(\mathrm{mg} / \mathrm{L})\end{array}$ & $\begin{array}{c}\text { Dissolved } \\
\text { oxygen } \\
\text { (percent } \\
\text { satura- } \\
\text { tion) }\end{array}$ \\
\hline
\end{tabular}

\begin{tabular}{|c|c|c|c|c|c|c|c|c|c|}
\hline \multicolumn{10}{|l|}{ June } \\
\hline 29 & 1532 & 0.5 & 600 & 416 & 7.6 & 27.8 & $\cdots$ & 7.2 & 94 \\
\hline 29 & 1532 & 3.0 & 600 & 417 & 75 & 27.6 & 15 & 71 & $\begin{array}{l}94 \\
0\end{array}$ \\
\hline 29 & 1534 & 50 & 600 & 11 & 75 & 277 & & 60 & 92 \\
\hline & & & ( & 413 & & & & . & 90 \\
\hline 29 & 1533 & 10 & 600 & 421 & 7.5 & 27.5 & $\cdots$ & 7.1 & 92 \\
\hline 29 & 1534 & 15 & 600 & 423 & 7.5 & 27.5 & $\cdots$ & 6.8 & 88 \\
\hline 29 & 1535 & 20 & 600 & 423 & 7.5 & 27.5 & - & 6.8 & 87 \\
\hline 29 & 1535 & 25 & 600 & 422 & 7.5 & 27.5 & - & 6.8 & 87 \\
\hline 29 & 1536 & 30 & 600 & 425 & 7.5 & 27.5 & $\cdots$ & 6.7 & 87 \\
\hline 29 & 1526 & 0.2 & 800 & 422 & 7.7 & 28.1 & - & 7.7 & 100 \\
\hline 29 & 1527 & 3.1 & 800 & 424 & 7.7 & 28.0 & $\cdots$ & 7.4 & 96 \\
\hline 29 & 1527 & 4.5 & 800 & 423 & 7.6 & 27.9 & $\cdots$ & 7.4 & 96 \\
\hline 29 & 1528 & 9.6 & 800 & 423 & 7.6 & 27.7 & - & 7.2 & 93 \\
\hline 29 & 1528 & 15 & 800 & 425 & 7.5 & 27.6 & $\cdots$ & 7.0 & 90 \\
\hline 29 & 1529 & 20 & 800 & 422 & 7.5 & 27.7 & $\cdots$ & 7.0 & 90 \\
\hline 29 & 1529 & 25 & 800 & 424 & 7.5 & 27.6 & - & 6.9 & 90 \\
\hline 29 & 1530 & 27 & 800 & 423 & 7.5 & 27.7 & $\cdots$ & 6.9 & 90 \\
\hline July & & & & & & & & & \\
\hline 13 & 0555 & 0.5 & 300 & 468 & 7.7 & 27.1 & $\cdots$ & 7.5 & 96 \\
\hline 13 & 0556 & 3.1 & 300 & 471 & 7.7 & 27.2 & $\cdots$ & 7.5 & 96 \\
\hline 13 & 0556 & 5.3 & 300 & 472 & 7.7 & 27.2 & $\cdots$ & 7.5 & 96 \\
\hline 13 & 0557 & 9.9 & 300 & 473 & 7.7 & 27.2 & $\cdots$ & 7.5 & 96 \\
\hline 13 & 0557 & 15 & 300 & 471 & 7.7 & 27.2 & $\cdots$ & 7.5 & 96 \\
\hline 13 & 0558 & 20 & 300 & 473 & 7.7 & 27.2 & $\cdots$ & 7.4 & 95 \\
\hline 13 & 0558 & 25 & 300 & 472 & 7.7 & 27.2 & $\cdots$ & 7.4 & 95 \\
\hline 13 & 0559 & 26 & 300 & 465 & 7.7 & 27.2 & $\cdots$ & 7.4 & 95 \\
\hline 13 & 0601 & 0.5 & 600 & 471 & 7.7 & 27.2 & - & 7.5 & 96 \\
\hline 13 & 0601 & 3.3 & 600 & 469 & 7.7 & 27.2 & $\cdots$ & 7.5 & 96 \\
\hline 13 & 0602 & 5.2 & 600 & 470 & 7.7 & 27.2 & - & 7.5 & 96 \\
\hline 13 & 0602 & 9.9 & 600 & 471 & 7.7 & 27.2 & $\cdots$ & 7.4 & 95 \\
\hline 13 & 0603 & 15 & 600 & 469 & 7.7 & 27.2 & - & 7.5 & 95 \\
\hline 13 & 0603 & 20 & 600 & 473 & 7.7 & 27.2 & - - & 7.4 & 95 \\
\hline 13 & 0604 & 25 & 600 & 470 & 7.7 & 27.2 & - - & 7.4 & 94 \\
\hline 13 & 0605 & 30 & 600 & 474 & 7.7 & 27.2 & $\cdots$ & 7.4 & 94 \\
\hline 13 & 0605 & 35 & 600 & 467 & 7.7 & 27.2 & - & 7.4 & 95 \\
\hline 13 & 0606 & 36 & 600 & 472 & 7.7 & 27.2 & $\cdots$ & 7.4 & 95 \\
\hline
\end{tabular}


Table 24. Water-quality data for station 391447081414201, Ohio River at river mile 192.9, June to October, 1995, Continued.

$\mathrm{Et}=\mathrm{feet} ; \mu \mathrm{S} / \mathrm{cm}=$ mucrosiemens per centımeter; ${ }^{\circ} \mathrm{C}=$ degrees Celsius;

$\mathrm{mg} / \mathrm{L}=\mathrm{milligrams}$ per liter: - = data not collected]

\begin{tabular}{|c|c|c|c|c|c|c|c|c|c|}
\hline Date & Time & $\begin{array}{l}\text { Sampling } \\
\text { depth } \\
\text { (ft) }\end{array}$ & $\begin{array}{c}\text { Sample } \\
\text { location } \\
\text { (Et Erom } \\
\text { left bank) }\end{array}$ & $\begin{array}{l}\text { Specific } \\
\text { conduct- } \\
\text { ance } \\
(\mu \mathrm{S} / \mathrm{cm})\end{array}$ & $\begin{array}{l}\mathrm{pH} \\
\text { (stan- } \\
\text { dard } \\
\text { undts) }\end{array}$ & $\begin{array}{c}\text { Temper- } \\
\text { ature, } \\
\text { water } \\
\left\langle{ }^{\circ} \mathrm{C}\right\rangle\end{array}$ & $\begin{array}{l}\text { Trans- } \\
\text { parency } \\
\text { (SecchI } \\
\text { disk) } \\
\text { (ft) }\end{array}$ & $\begin{array}{l}\text { Dissolved } \\
\text { oxygen } \\
(\mathrm{mg} / \mathrm{L})\end{array}$ & $\begin{array}{c}\text { Dissolved } \\
\text { oxygen } \\
\text { (percent } \\
\text { satura- } \\
\text { tion) }\end{array}$ \\
\hline
\end{tabular}

\begin{tabular}{|c|c|c|c|c|c|c|c|c|c|}
\hline $\begin{array}{r}\text { Ju1Y } \\
13\end{array}$ & 0608 & 0.5 & 800 & & 77 & & & & \\
\hline 13 & 0608 & 3.2 & $\begin{array}{l}800 \\
800\end{array}$ & $\begin{array}{l}470 \\
473\end{array}$ & 7.7 & $\begin{array}{l}27 \cdot 1 \\
27.2\end{array}$ & $\ldots$ & $\begin{array}{l}7.4 \\
7.4\end{array}$ & $\begin{array}{l}95 \\
95\end{array}$ \\
\hline 13 & 0609 & 5.2 & 800 & $\begin{array}{l}413 \\
470\end{array}$ & 7.7 & 27.2 & $\ldots$ & $\begin{array}{l}7.4 \\
7.4\end{array}$ & $\begin{array}{l}95 \\
95\end{array}$ \\
\hline 13 & 0609 & 10 & 800 & 469 & 7.7 & 27.2 & $\ldots$ & 7.4 & 95 \\
\hline 13 & 0610 & 15 & 800 & 474 & 7.7 & 27.2 & - & 7.4 & 94 \\
\hline 13 & 0610 & 20 & 800 & 468 & 7.7 & 27.2 & -- & 7.2 & 93 \\
\hline 13 & 0611 & 25 & 800 & 468 & 7.7 & 27.2 & - - & 7.2 & 93 \\
\hline 13 & 0611 & 30 & 800 & 471 & 7.6 & 27.2 & $\cdots$ & 7.2 & 92 \\
\hline 13 & 0612 & 31 & 800 & 471 & 7.6 & 27.2 & $\cdots$ & 7.2 & 92 \\
\hline 13 & 1804 & 0.6 & 300 & 468 & 9.0 & 31.4 & $\cdots$ & 13.3 & 183 \\
\hline 13 & 1805 & 2.9 & 300 & 472 & 8.9 & 30.5 & $\cdots$ & 12.7 & 172 \\
\hline 13 & 1805 & 5.1 & 300 & 481 & 8.1 & 28.2 & $\cdots$ & 8.8 & 115 \\
\hline 13 & 1806 & 10 & 300 & 475 & 7.7 & 27.5 & -- & 7.5 & 96 \\
\hline 13 & 1807 & 15 & 300 & 471 & 7.7 & 27.4 & $\cdots$ & 7.0 & 90 \\
\hline 13 & 1807 & 20 & 300 & 470 & 7.6 & 27.3 & $\cdots$ & 6.8 & 87 \\
\hline 13 & 1808 & 25 & 300 & 469 & 7.6 & 27.3 & - - & 6.7 & 86 \\
\hline 13 & 1752 & 0.7 & 600 & 469 & 8.8 & 30.2 & $\cdots$ & 12.6 & 170 \\
\hline 13 & 1752 & 3.2 & 600 & 471 & 8.6 & 29.1 & $\cdots$ & 10.9 & 144 \\
\hline 13 & 1753 & 4.8 & 600 & 471 & 8.2 & 28.2 & $\cdots$ & 9.1 & 119 \\
\hline 13 & 1754 & 9.8 & 600 & 468 & 7.8 & 27.5 & $\cdots$ & 7.7 & 9 \\
\hline 13 & 1754 & 15 & 600 & 468 & 7.8 & 27.5 & $\cdots$ & 7.6 & 98 \\
\hline 13 & 1755 & 20 & 600 & 467 & 7.7 & 27.4 & $\cdots$ & 7.4 & 95 \\
\hline 13 & 1756 & 25 & 600 & 469 & 7.7 & 27.3 & $\cdots$ & 7.1 & 91 \\
\hline 13 & 1756 & 30 & 600 & 468 & 7.6 & 27.2 & $-\cdot$ & 6.7 & 86 \\
\hline 13 & 1757 & 35 & 600 & 466 & 7.6 & 27.2 & $\cdots$ & 6.6 & 85 \\
\hline 13 & 1758 & 37 & 600 & 468 & 7.6 & 27.2 & $\cdots$ & 6.6 & 84 \\
\hline 13 & 1744 & 0.7 & 800 & 471 & 8.8 & 29.9 & $\cdots$ & 12.0 & 162 \\
\hline 13 & 1744 & 3.1 & 800 & 470 & 8.7 & 29.1 & $\cdots$ & 12.0 & 159 \\
\hline 13 & 1745 & 4.9 & 800 & 468 & 8.6 & 28.8 & $\cdots$ & 11.6 & 152 \\
\hline 13 & 1746 & 10 & 800 & 469 & 8.0 & 27.8 & $\cdots$ & 8.6 & 112 \\
\hline 13 & 1747 & 15 & 800 & 469 & 7.9 & 27.6 & $\cdots$ & 8.0 & 103 \\
\hline 13 & 1748 & 20 & 800 & 470 & 7.8 & 27.6 & $\cdots$ & 7.9 & 101 \\
\hline 13 & 1747 & 25 & 800 & 468 & 7.6 & 27.3 & $\cdots$ & 6.9 & 8 \\
\hline 13 & 1749 & 28 & 800 & 469 & 7.6 & 27.2 & $\cdots$ & 6.7 & 8 \\
\hline
\end{tabular}


Table 24. Water-quality data for station 391447081414201, Ohio River at river mile 192.9, June to October, 1995, Continued.

$\mathrm{ft}=\mathrm{feet} ; \mu \mathrm{s} / \mathrm{cm}=$ microsiemens per centimeter; ${ }^{\circ} \mathrm{C}=$ degrees Celsius;

$\mathrm{mg} / \mathrm{L}=\mathrm{mili}$ igrams per liter; $\cdots$ = data not collected]

\begin{tabular}{|c|c|c|c|c|c|c|c|c|c|}
\hline Date & Time & $\begin{array}{l}\text { Sampling } \\
\text { depth } \\
\text { (ft) }\end{array}$ & $\begin{array}{c}\text { Sample } \\
\text { location } \\
\text { (ft from } \\
\text { left bank) }\end{array}$ & $\begin{array}{l}\text { Speciflc } \\
\text { conduct- } \\
\text { ance } \\
(\mu S / \mathrm{cm})\end{array}$ & $\begin{array}{l}\mathrm{pH} \\
\text { (stan- } \\
\text { dard } \\
\text { units) }\end{array}$ & $\begin{array}{c}\text { Temper- } \\
\text { ature, } \\
\text { water } \\
\left({ }^{\circ} \mathrm{C}\right)\end{array}$ & $\begin{array}{l}\text { Trans- } \\
\text { parency } \\
\text { (Secchi } \\
\text { disk) } \\
\text { (Et) }\end{array}$ & $\begin{array}{l}\text { Dissolved } \\
\text { oxygen } \\
(\mathrm{mg} / \mathrm{L})\end{array}$ & $\begin{array}{c}\text { Dissolved } \\
\text { oxygen } \\
\text { (percent } \\
\text { satura- } \\
\text { tion) }\end{array}$ \\
\hline
\end{tabular}

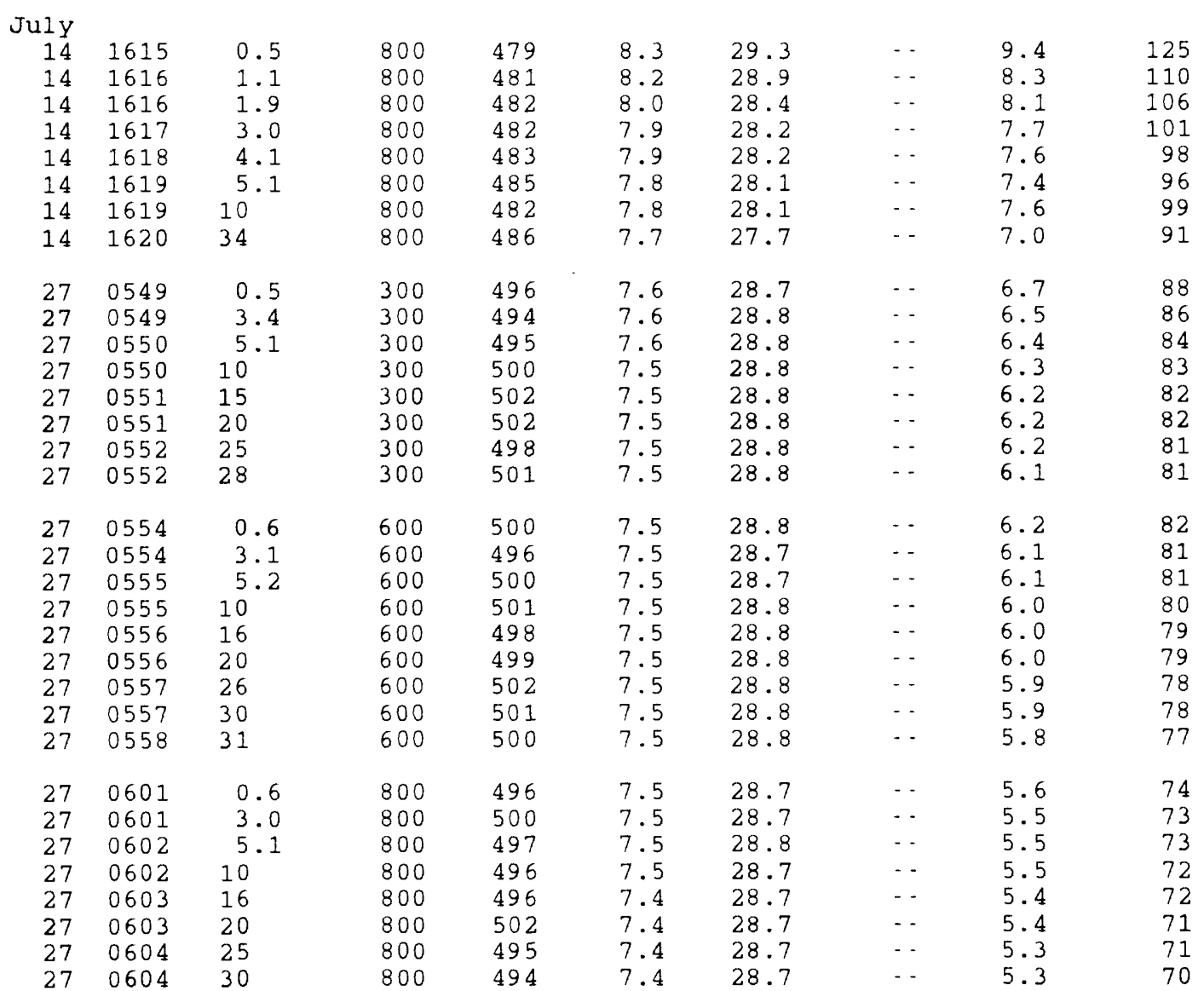


Table 24. Water-quality data for station 391447081414201, Ohio River at river mile 192.9, June to October, 1995, Continued.

ft $=$ feet; $\mu \mathrm{s} / \mathrm{cm}=$ microsiemens per centimeter; ${ }^{\circ} \mathrm{C}=$ degrees Celsius; $\mathrm{mg} / \mathrm{L}=$ milligrams per liter; - - = data not collected]

\begin{tabular}{|c|c|c|c|c|c|c|c|c|c|}
\hline Date & Time & $\begin{array}{l}\text { Sampling } \\
\text { depth } \\
(E t)\end{array}$ & $\begin{array}{l}\text { Sample } \\
\text { location } \\
\text { (Et from } \\
\text { left bank) }\end{array}$ & $\begin{array}{l}\text { specific } \\
\text { conduct- } \\
\text { ance } \\
(\mu \mathrm{s} / \mathrm{cm})\end{array}$ & $\begin{array}{l}\text { pH } \\
\text { (stan- } \\
\text { dard } \\
\text { units) }\end{array}$ & $\begin{array}{c}\text { Temper- } \\
\text { ature, } \\
\text { water } \\
\left({ }^{\circ} \mathrm{C}\right)\end{array}$ & $\begin{array}{l}\text { Trans- } \\
\text { parency } \\
\text { (secchI } \\
\text { disk) } \\
\text { (Et) }\end{array}$ & $\begin{array}{l}\text { Dissolved } \\
\text { oxygen } \\
(\mathrm{mg} / \mathrm{L})\end{array}$ & $\begin{array}{c}\text { Dissolved } \\
\text { oxygen } \\
\text { (percent } \\
\text { satura- } \\
\text { tion) }\end{array}$ \\
\hline
\end{tabular}

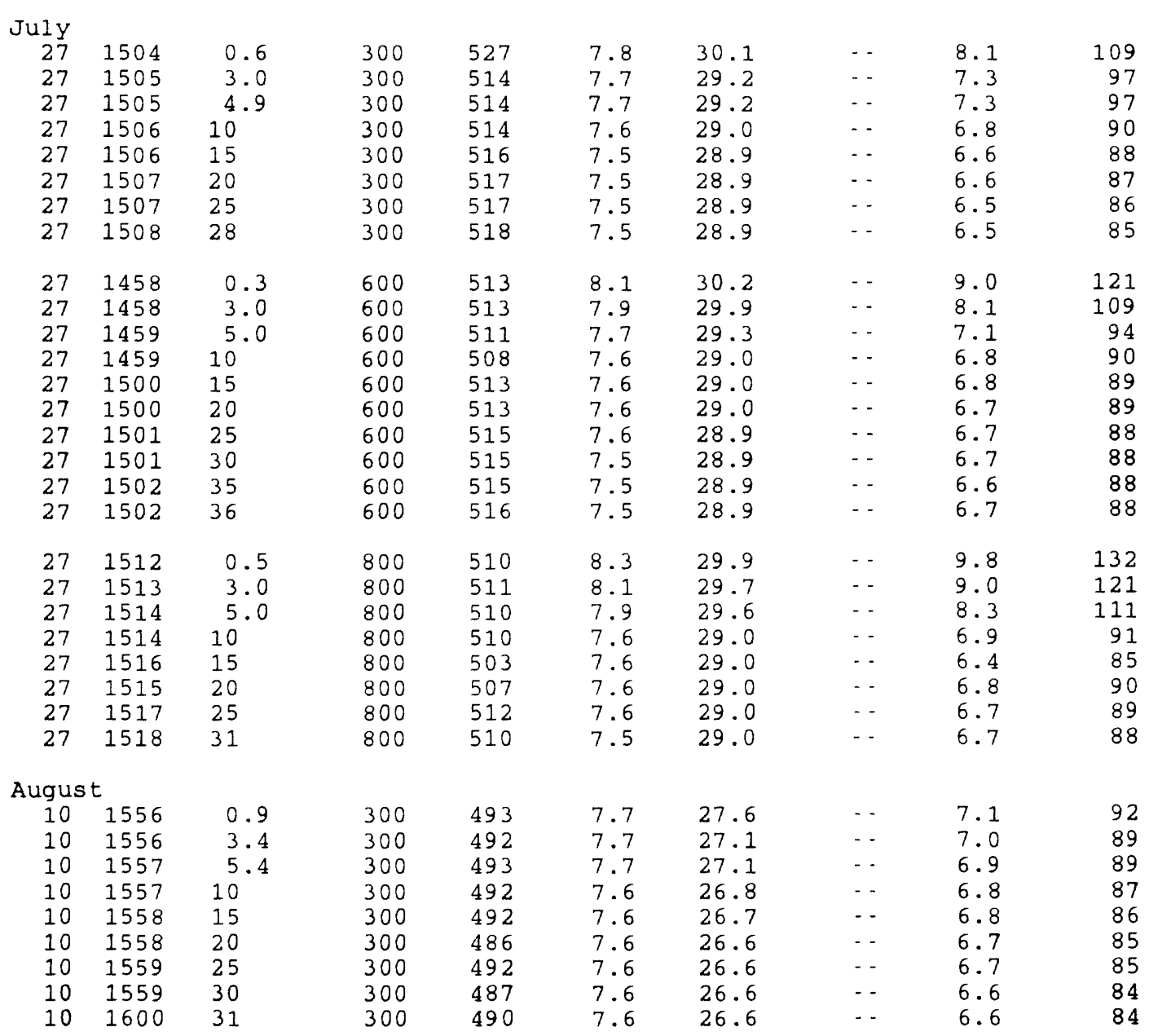


Table 24. Water-quality data for station 391447081414201, Ohio River at river mile 192.9, June to october, 1995, Continued.

[ft $=$ teet; $\mu \mathrm{S} / \mathrm{cm}=$ microsiemens per centimeter $;{ }^{\circ} \mathrm{C}=$ degrees Celsius; $\mathrm{mg} / \mathrm{L}=\mathrm{milligrams}$ per Iiter;. - = data not collected]

\begin{tabular}{|c|c|c|c|c|c|c|c|c|c|}
\hline Date & Time & $\begin{array}{l}\text { Sampling } \\
\text { depth } \\
\text { (ft) }\end{array}$ & $\begin{array}{l}\text { Sample } \\
\text { location } \\
\text { (tt from } \\
\text { left bank) }\end{array}$ & $\begin{array}{l}\text { Specific } \\
\text { conduct- } \\
\text { ance } \\
(\mu \mathrm{s} / \mathrm{cm})\end{array}$ & $\begin{array}{l}\text { pH } \\
\text { (stan- } \\
\text { dard } \\
\text { unzts) }\end{array}$ & $\begin{array}{c}\text { Temper- } \\
\text { ature, } \\
\text { water } \\
\left({ }^{\circ} \mathrm{C}\right)\end{array}$ & $\begin{array}{c}\text { Trans- } \\
\text { parency } \\
\text { (Secchl } \\
\text { disk) } \\
\text { (tt) }\end{array}$ & $\begin{array}{l}\text { Dissolved } \\
\text { oxygen } \\
(\mathrm{mg} / \mathrm{L})\end{array}$ & $\begin{array}{c}\text { Dissolved } \\
\text { oxygen } \\
\text { (percent } \\
\text { satura- } \\
\text { tion) }\end{array}$ \\
\hline
\end{tabular}

\begin{tabular}{|c|c|c|c|c|}
\hline \multicolumn{5}{|c|}{ August } \\
\hline 10 & 1600 & 0.8 & 600 & 49 \\
\hline 10 & 1600 & 3.5 & 600 & 49 \\
\hline 10 & 1601 & 5.1 & 600 & \\
\hline 10 & 1602 & 10 & 600 & 45 \\
\hline 10 & 1602 & 15 & 600 & 45 \\
\hline 10 & 1603 & 20 & 600 & \\
\hline 10 & 1603 & 25 & 600 & 4 \\
\hline 10 & 1604 & 30 & 600 & \\
\hline 10 & 1604 & 34 & 600 & \\
\hline 10 & 1605 & 37 & 600 & \\
\hline 10 & 1607 & 1.1 & 800 & \\
\hline 10 & 1607 & 3.6 & 800 & \\
\hline 10 & 1608 & 5.4 & 800 & 49 \\
\hline 10 & 1609 & 10 & 800 & 48 \\
\hline 10 & 1609 & 15 & 800 & 49 \\
\hline 10 & 1610 & 20 & 800 & \\
\hline 10 & 1610 & 25 & 800 & \\
\hline 10 & 1611 & 29 & 800 & \\
\hline 24 & 0758 & 0.5 & 300 & \\
\hline 24 & 0758 & 3.3 & 300 & \\
\hline 24 & 0759 & 5.2 & 300 & \\
\hline 24 & 0759 & 10 & 300 & 50 \\
\hline 24 & 0800 & 15 & 300 & 5 \\
\hline 24 & 0800 & 20 & 300 & 50 \\
\hline 24 & 0801 & 25 & 300 & \\
\hline 24 & 0801 & 29 & 300 & \\
\hline 24 & 0752 & 1.0 & 600 & \\
\hline 24 & 0752 & 3.3 & 600 & \\
\hline 24 & 0753 & 5.1 & 600 & \\
\hline 24 & 0753 & 10 & 600 & \\
\hline 24 & 0754 & 15 & 600 & 4 \\
\hline 24 & 0754 & 20 & 600 & \\
\hline 24 & 0755 & 25 & 600 & 4 \\
\hline 24 & 0755 & 30 & 600 & 4 \\
\hline 24 & 0756 & 34 & 600 & \\
\hline
\end{tabular}

\begin{tabular}{|c|c|c|c|}
\hline 7.7 & 27.9 & - & 7.2 \\
\hline 7.7 & 27.2 & $\cdots$ & 6.9 \\
\hline 7.6 & 26.9 & $\cdots$ & 6.8 \\
\hline 7.6 & 26.7 & $\cdots$ & 6.8 \\
\hline 7.6 & 26.7 & $\cdots$ & 6.7 \\
\hline 7.6 & 26.6 & - & 6.7 \\
\hline 7.6 & 26.6 & $\ldots$ & 6.7 \\
\hline 7.6 & 26.6 & $\ldots$ & 6.7 \\
\hline 7.6 & 26.6 & $\cdots$ & 6.7 \\
\hline 7.6 & 26.6 & $\cdots$ & 6.6 \\
\hline 7.7 & 27.6 & $\cdots$ & 7.0 \\
\hline 7.7 & 26.9 & $\cdots$ & 6.8 \\
\hline 7.6 & 26.7 & $\cdots$ & 6.6 \\
\hline 7.6 & 26.7 & $\cdots$ & 6.6 \\
\hline 7.6 & 26.7 & $\cdots$ & 6.5 \\
\hline 7.6 & 26.7 & $\ldots$ & 6.6 \\
\hline 7.6 & 26.7 & $\cdots$ & 6.5 \\
\hline 7.6 & 26.6 & $\cdots$ & 6.6 \\
\hline 8.0 & 29.5 & $\cdots$ & 7.4 \\
\hline 8.0 & 29.6 & $\cdots$ & 7.4 \\
\hline 8.0 & 29.6 & $\cdots$ & 7.4 \\
\hline 8.0 & 29.6 & $\cdots$ & 7.4 \\
\hline 8.0 & 29.6 & - & 7.4 \\
\hline 8.0 & 29.6 & $\cdots$ & 7.3 \\
\hline 8.0 & 29.6 & $\cdots$ & 7.2 \\
\hline 8.0 & 29.5 & $\cdots$ & 7.2 \\
\hline 7.9 & 29.5 & $\cdots$ & 7.4 \\
\hline 7.9 & 29.5 & $\cdots$ & 7.3 \\
\hline 7.9 & 29.5 & $\cdots$ & 7.3 \\
\hline 7.9 & 29.5 & $\cdots$ & 7.3 \\
\hline 7.9 & 29.5 & $\cdots$ & 7.2 \\
\hline 7.9 & 29.6 & $\cdots$ & 7.2 \\
\hline 7.9 & 29.6 & $\cdots$ & 7.2 \\
\hline 7.9 & 29.5 & $\cdots$ & 7.3 \\
\hline 7.9 & 29.5 & $\cdots$ & 7.2 \\
\hline
\end{tabular}


Table 24. Water-quality data for station 391447081414201, Ohio River at river mile 192.9, June to October, 1995, Continued.

$\left[\mathrm{ft}=\mathrm{feet} ; \mu \mathrm{S} / \mathrm{cm}=\right.$ microsıemens per centımeter $;{ }^{\circ} \mathrm{C}=$ degrees Celsius; $\mathrm{mg} / \mathrm{L}=$ milligrams per 11ter; $\cdot$ - = data not collected]

\begin{tabular}{|c|c|c|c|c|c|c|c|c|c|}
\hline Date & Time & $\begin{array}{l}\text { Sampling } \\
\text { depth } \\
\text { (ft) }\end{array}$ & $\begin{array}{l}\text { Sample } \\
\text { location } \\
\text { (Et from } \\
\text { left bank) }\end{array}$ & $\begin{array}{l}\text { Specific } \\
\text { conduct- } \\
\text { ance } \\
(\mu \mathrm{S} / \mathrm{cm})\end{array}$ & $\begin{array}{l}\mathrm{pH} \\
\text { (stan- } \\
\text { dard } \\
\text { units) }\end{array}$ & $\begin{array}{c}\text { Temper- } \\
\text { ature, } \\
\text { water } \\
\left({ }^{\circ} \mathrm{C}\right)\end{array}$ & $\begin{array}{l}\text { Trans. } \\
\text { parency } \\
\text { (Secchl } \\
\text { disk) } \\
\text { (tt) }\end{array}$ & $\begin{array}{l}\text { Dissolved } \\
\text { oxygen } \\
\text { (mg/L) }\end{array}$ & $\begin{array}{c}\text { Dissolved } \\
\text { oxygen } \\
\text { (percent } \\
\text { satura- } \\
\text { tron) }\end{array}$ \\
\hline
\end{tabular}

\begin{tabular}{|c|c|c|c|c|c|c|c|c|c|}
\hline \multicolumn{10}{|c|}{ August } \\
\hline 24 & 0746 & 0.2 & 800 & 493 & 7.9 & 29.5 & $\cdots$ & 7.3 & 98 \\
\hline 24 & 0746 & 3.2 & 800 & 491 & 7.9 & 29.5 & $\cdots$ & 7.3 & 98 \\
\hline 24 & 0747 & 5.1 & 800 & 490 & 7.9 & 29.5 & $\cdots$ & 7.3 & 98 \\
\hline 24 & 0747 & 9.8 & 800 & 488 & 7.9 & 29.5 & $\cdots$ & 7.3 & 98 \\
\hline 24 & 0748 & 15 & 800 & 494 & 7.9 & 29.5 & $\cdots$ & 7.3 & 97 \\
\hline 24 & 0749 & 20 & 800 & 491 & 7.9 & 29.5 & $\cdots$ & 7.2 & 95 \\
\hline 24 & 0749 & 25 & 800 & 488 & 7.9 & 29.5 & $\cdots$ & 7.1 & 94 \\
\hline 24 & 0750 & 29 & 800 & 495 & 7.9 & 29.5 & $\cdots$ & 7.0 & 94 \\
\hline 24 & 1402 & 0.5 & 300 & 507 & 8.8 & 31.4 & $\cdots$ & 12.3 & 169 \\
\hline 24 & 1402 & 3.3 & 300 & 509 & 8.3 & 29.9 & - & 8.8 & 118 \\
\hline 24 & 1403 & 5.4 & 300 & 512 & 8.1 & 29.7 & $\cdots$ & 7.9 & 105 \\
\hline 24 & 1403 & 10 & 300 & 513 & 8.0 & 29.6 & $\cdots$ & 7.2 & 97 \\
\hline 24 & 1404 & 15 & 300 & 519 & 8.0 & 29.6 & $\cdots$ & 6.9 & 92 \\
\hline 24 & 1405 & 20 & 300 & 521 & 7.9 & 29.5 & $\cdots$ & 6.7 & 90 \\
\hline 24 & 1405 & 25 & 300 & 518 & 7.9 & 29.5 & $\cdots$ & 6.8 & 90 \\
\hline 24 & 1406 & 29 & 300 & 517 & 7.9 & 29.5 & $\cdots$ & 6.7 & 89 \\
\hline 24 & 1351 & 0.5 & 600 & 509 & 8.2 & 29.9 & $\cdots$ & 8.3 & 112 \\
\hline 24 & 1351 & 3.0 & 600 & 508 & 8.2 & 29.8 & $\cdots$ & 7.5 & 101 \\
\hline 24 & 1352 & 5.1 & 600 & 506 & 8.0 & 29.6 & $\cdots$ & 7.5 & 100 \\
\hline 24 & 1352 & 10 & 600 & 509 & 8.0 & 29.6 & $\cdots$ & 7.4 & 98 \\
\hline 24 & 1353 & 15 & 600 & 508 & 8.0 & 29.6 & $\cdots$ & 7.3 & 98 \\
\hline 24 & 1353 & 20 & 600 & 510 & 8.0 & 29.6 & $\cdots$ & 7.3 & 97 \\
\hline 24 & 1354 & 25 & 600 & 509 & 8.0 & 29.5 & $\cdots$ & 7.1 & 95 \\
\hline 24 & 1354 & 30 & 600 & 507 & 8.0 & 29.6 & $\cdots$ & 7.1 & 94 \\
\hline 24 & 1355 & 35 & 600 & 507 & 8.0 & 29.6 & $\cdots$ & 7.1 & 95 \\
\hline 24 & 1355 & 36 & 600 & 510 & 8.0 & 29.6 & $\cdots$ & 7.1 & 95 \\
\hline 24 & 1340 & 0.3 & 800 & 505 & 8.7 & 31.4 & $\cdots$ & 11.5 & 159 \\
\hline 24 & 1343 & 3.2 & 800 & 506 & 8.7 & 30.4 & $\cdots$ & 12.3 & 166 \\
\hline 24 & 1344 & 5.2 & 800 & 508 & 8.4 & 29.8 & $\cdots$ & 9.4 & 126 \\
\hline 24 & 1347 & 9.8 & 800 & 511 & 8.0 & 29.6 & $\cdots$ & 7.4 & 99 \\
\hline 24 & 1347 & 15 & 800 & 508 & 8.0 & 29.6 & $\cdots$ & 7.2 & 96 \\
\hline 24 & 1348 & 20 & 800 & 510 & 8.0 & 29.6 & $\cdots$ & 7.2 & 96 \\
\hline 24 & 1349 & 25 & 800 & 510 & 8.0 & 29.6 & $\cdots$ & 7.2 & 96 \\
\hline
\end{tabular}


Table 24. Water-quality data for station 391447081414201, Ohio River at river mile 192.9, June to October, 1995, Continued.

$\mathrm{ft}=$ feet $; \mu \mathrm{s} / \mathrm{cm}=$ microslemens per centimeter $;{ }^{\circ} \mathrm{C}=$ degrees Celsius:

$\mathrm{mg} / \mathrm{L}=$ miliigrams per liter; - = data not collected

\begin{tabular}{|c|c|c|c|c|c|c|c|c|c|}
\hline Date & Time & $\begin{array}{l}\text { Sampling } \\
\text { depth } \\
\text { (ft) }\end{array}$ & $\begin{array}{l}\text { Sample } \\
\text { location } \\
\text { (ft from } \\
\text { left bank) }\end{array}$ & $\begin{array}{l}\text { Specif Ic } \\
\text { conduct - } \\
\text { ance } \\
(\mu \mathrm{s} / \mathrm{cm})\end{array}$ & $\begin{array}{l}\mathrm{pH} \\
\text { (stan - } \\
\text { dard } \\
\text { units) }\end{array}$ & $\begin{array}{c}\text { Temper- } \\
\text { ature, } \\
\text { water } \\
\left({ }^{\circ} \mathrm{C}\right)\end{array}$ & $\begin{array}{l}\text { Trans - } \\
\text { parency } \\
\text { (secchl } \\
\text { disk) } \\
\text { (ft) }\end{array}$ & $\begin{array}{l}\text { Dissolved } \\
\text { oxygen } \\
(\mathrm{mg} / \mathrm{L})\end{array}$ & $\begin{array}{c}\text { Dissolved } \\
\text { oxygen } \\
\text { (percent } \\
\text { satura- } \\
\text { tion) }\end{array}$ \\
\hline
\end{tabular}

\begin{tabular}{|c|c|c|c|c|c|c|c|c|c|}
\hline \multicolumn{10}{|c|}{ September } \\
\hline 06 & 0602 & 1.0 & 300 & 537 & 7.6 & 27.5 & $\cdots$ & 6.6 & 85 \\
\hline 06 & 0602 & 3.1 & 300 & 541 & 7.6 & 27.5 & - & 6.7 & 85 \\
\hline 06 & 0603 & 5.0 & 300 & 536 & 7.6 & 27.5 & -- & 6.7 & 86 \\
\hline 06 & 0603 & 10 & 300 & 535 & 7.6 & 27.5 & $\cdots$ & 6.6 & 84 \\
\hline 06 & 0604 & 16 & 300 & 540 & 7.6 & 27.5 & $\cdots$ & 6.6 & 85 \\
\hline 06 & 0604 & 20 & 300 & 538 & 7.6 & 27.5 & $-\cdot$ & 6.6 & 85 \\
\hline 06 & 0605 & 25 & 300 & 549 & 7.6 & 27.5 & $\cdots$ & 6.6 & 85 \\
\hline 06 & 0607 & 0.3 & 600 & 540 & 7.6 & 27.4 & $\cdots$ & 6.8 & 87 \\
\hline 06 & 0607 & 2.1 & 600 & 546 & 7.6 & 27.5 & $\cdots$ & 6.7 & 86 \\
\hline 06 & 0608 & 5.0 & 600 & 540 & 7.6 & 27.5 & -- & 6.8 & 87 \\
\hline 06 & 0608 & 10 & 600 & 541 & 7.6 & 27.5 & -- & 6.8 & 87 \\
\hline 06 & 0609 & 15 & 600 & 540 & 7.6 & 27.5 & $\cdots$ & 6.8 & 87 \\
\hline 06 & 0609 & 20 & 600 & 547 & 7.6 & 27.5 & $\cdots$ & 6.8 & 87 \\
\hline 06 & 0610 & 26 & 600 & 541 & 7.6 & 27.5 & -- & 6.8 & 87 \\
\hline 06 & 0610 & 28 & 600 & 542 & 7.6 & 27.5 & $\cdots$ & 6.6 & 85 \\
\hline 06 & 0613 & 1.0 & 800 & 542 & 7.6 & 27.5 & - & 6.9 & 88 \\
\hline 06 & 0613 & 3.3 & 800 & 540 & 7.6 & 27.5 & $\cdots$ & 6.9 & 88 \\
\hline 06 & 0614 & 6.2 & 800 & 540 & 7.7 & 27.5 & - - & 6.9 & 89 \\
\hline 06 & 0614 & 10 & 800 & 544 & 7.7 & 27.5 & $\cdots$ & 6.9 & 88 \\
\hline 06 & 0615 & 16 & 800 & 544 & 7.7 & 27.5 & $\cdots$ & 6.9 & 88 \\
\hline 06 & 0615 & 20 & 800 & 545 & 7.7 & 27.5 & $\cdots$ & 6.9 & 89 \\
\hline 06 & 0616 & 25 & 800 & 542 & 7.7 & 27.5 & $\cdots$ & 6.9 & 89 \\
\hline 06 & 0616 & 29 & 800 & 547 & 7.7 & 27.5 & -- & 6.8 & 87 \\
\hline 06 & 1535 & 0.2 & 300 & 541 & 8.4 & 30.0 & -- & 10.6 & 142 \\
\hline 06 & 1535 & 2.9 & 300 & 540 & 8.4 & 29.4 & -- & 10.4 & 138 \\
\hline 06 & 1536 & 4.9 & 300 & 540 & 8.3 & 29.2 & $\cdots$ & 10.2 & 134 \\
\hline 06 & 1536 & 9.5 & 300 & 545 & 7.6 & 27.5 & - & 6.7 & 86 \\
\hline 06 & 1537 & 15 & 300 & 542 & 7.6 & 27.4 & $\cdots$ & 6.5 & 83 \\
\hline 06 & 1537 & 20 & 300 & 543 & 7.5 & 27.4 & $\cdots$ & 6.5 & 84 \\
\hline 06 & 1538 & 25 & 300 & 545 & 7.5 & 27.4 & - - & 6.5 & 8 \\
\hline 06 & 1538 & 28 & 300 & 539 & 7.5 & 27.4 & $\cdots$ & 6.5 & 83 \\
\hline
\end{tabular}


Table 24. Water-quality data for station 391447081414201, Ohio River at river mile 192.9, June to October, 1995, Continued.

[ft = feet; $\mu \mathrm{s} / \mathrm{cm}=$ microslemens per centimeter; ${ }^{\circ} \mathrm{C}=$ degrees Celsius; $\mathrm{mg} / \mathrm{L}=\mathrm{milligrams}$ per liter; - = data not collected]

\begin{tabular}{|c|c|c|c|c|c|c|c|c|c|}
\hline Date & Time & $\begin{array}{l}\text { Sampling } \\
\text { depth } \\
\text { ( } f t)\end{array}$ & $\begin{array}{c}\text { Sample } \\
\text { location } \\
\text { (Et trom } \\
\text { left bank) }\end{array}$ & $\begin{array}{l}\text { Specific } \\
\text { conduct- } \\
\text { ance } \\
(\mu \mathrm{s} / \mathrm{cm})\end{array}$ & $\begin{array}{l}\mathrm{pH} \\
\text { (stan- } \\
\text { dard } \\
\text { units) }\end{array}$ & $\begin{array}{c}\text { Temper- } \\
\text { ature, } \\
\text { water } \\
\left({ }^{\circ} \mathrm{C}\right)\end{array}$ & $\begin{array}{l}\text { Trans - } \\
\text { parency } \\
\text { (Secch } \\
\text { disk) } \\
\text { (Et) }\end{array}$ & $\begin{array}{c}\text { Dissolved } \\
\text { oxygen } \\
\text { (mg/L) }\end{array}$ & $\begin{array}{c}\text { Dissolved } \\
\text { oxygen } \\
\text { (percent } \\
\text { satura- } \\
\text { tion) }\end{array}$ \\
\hline
\end{tabular}

\begin{tabular}{|c|c|c|c|c|c|c|c|c|c|}
\hline \multicolumn{10}{|c|}{ September } \\
\hline 06 & 1529 & 0.2 & 600 & 540 & 8.5 & 29.9 & $\cdots$ & 10.8 & 144 \\
\hline 06 & 1530 & 2.9 & 600 & 539 & 8.6 & 28.6 & $\cdots$ & 10.2 & 133 \\
\hline 06 & 1530 & 5.0 & 600 & 543 & 7.9 & 27.9 & $\cdots$ & 8.2 & 106 \\
\hline 06 & 1531 & 9.5 & 600 & 543 & 7.6 & 27.5 & - & 6.8 & 87 \\
\hline 06 & 1531 & 15 & 600 & 543 & 7.6 & 27.4 & 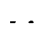 & 6.6 & 85 \\
\hline 06 & 1532 & 20 & 600 & 543 & 7.6 & 27.5 & - & 6.6 & 84 \\
\hline 06 & 1532 & 25 & 600 & 543 & 7.5 & 27.4 & $\cdots$ & 6.4 & 82 \\
\hline 06 & 1533 & 30 & 600 & 543 & 7.5 & 27.4 & $\cdots$ & 6.4 & 82 \\
\hline 06 & 1533 & 35 & 600 & 543 & 7.5 & 27.5 & $\cdots$ & 6.4 & 82 \\
\hline 06 & 1522 & 0.2 & 800 & 543 & 8.2 & 29.7 & $\cdots$ & 9.4 & 126 \\
\hline 06 & 1523 & 2.9 & 800 & 543 & 8.2 & 29.6 & $\cdots$ & 9.2 & 123 \\
\hline 06 & 1523 & 4.7 & 800 & 539 & 8.3 & 28.4 & $\cdots$ & 9.8 & 128 \\
\hline 06 & 1524 & 9.7 & 800 & 542 & 7.7 & 27.6 & $\cdots$ & 7.0 & 90 \\
\hline 06 & 1525 & 15 & 800 & 543 & 7.6 & 27.5 & - & 6.7 & 85 \\
\hline 06 & 1525 & 20 & 800 & 543 & 7.6 & 27.5 & - & 6.7 & 85 \\
\hline 06 & 1526 & 25 & 800 & 543 & 7.6 & 27.5 & $\ldots$ & 6.5 & 84 \\
\hline 06 & 1526 & 28 & 800 & 544 & 7.6 & 27.5 & $\cdots$ & 6.5 & 83 \\
\hline 20 & 0634 & 0.3 & 300 & 678 & 7.5 & 24.0 & $\cdots$ & 5.9 & 71 \\
\hline 20 & 0634 & 3.1 & 300 & 679 & 7.4 & 24.0 & $\cdots$ & 5.9 & 71 \\
\hline 20 & 0635 & 4.9 & 300 & 679 & 7.4 & 24.0 & $\cdots$ & 5.9 & 71 \\
\hline 20 & 0635 & 10 & 300 & 680 & 7.4 & 24.0 & $\cdots$ & 5.9 & 72 \\
\hline 20 & 0636 & 15 & 300 & 679 & 7.4 & 24.0 & $\cdots$ & 5.8 & 71 \\
\hline 20 & 0637 & 20 & 300 & 680 & 7.4 & 24.0 & $\cdots$ & 5.8 & 71 \\
\hline 20 & 0637 & 25 & 300 & 679 & 7.4 & 24.0 & -- & 5.8 & 70 \\
\hline 20 & 0638 & 30 & 300 & 680 & 7.4 & 24.0 & $\cdots$ & 5.7 & 69 \\
\hline 20 & 0620 & 0.3 & 600 & 680 & 7.5 & 24.0 & $\cdots$ & 5.9 & 72 \\
\hline 20 & 0620 & 2.7 & 600 & 680 & 7.4 & 24.0 & $\cdots$ & 5.9 & 72 \\
\hline 20 & 0621 & 5.0 & 600 & 680 & 7.4 & 24.0 & $\cdots$ & 5.9 & 72 \\
\hline 20 & 0621 & 9.8 & 600 & 680 & 7.4 & 24.1 & $\cdots$ & 5.9 & 72 \\
\hline 20 & 0622 & 14 & 600 & 680 & 7.4 & 24.1 & $\cdots$ & 5.9 & 72 \\
\hline 20 & 0622 & 19 & 600 & 680 & 7.4 & 24.1 & $\cdots$ & 5.9 & 72 \\
\hline 20 & 0623 & 26 & 600 & 680 & 7.4 & 24.1 & $\cdots$ & 5.9 & 71 \\
\hline 20 & 0623 & 30 & 600 & 680 & 7.4 & 24.1 & $\cdots$ & 5.8 & 71 \\
\hline 20 & 0624 & 34 & 600 & 680 & 7.4 & 24.1 & $\cdots$ & 5.8 & 71 \\
\hline
\end{tabular}


Table 24. Water-quality data for station 391447081414201, Ohio River at river mile 192.9, June to October, 1995, Continued.

[ft $=$ feet; $\mu \mathrm{s} / \mathrm{cm}=$ microsiemens per centimeter; ${ }^{\circ} \mathrm{C}=$ degrees Celsius;

$\mathrm{mg} / \mathrm{L}=\mathrm{milligrams}$ per liter; $\cdots$ = data not collected]

\begin{tabular}{|c|c|c|c|c|c|c|c|c|c|}
\hline Date & Time & $\begin{array}{l}\text { Sampling } \\
\text { depth } \\
\text { (ft) }\end{array}$ & $\begin{array}{c}\text { Sample } \\
\text { location } \\
\text { (ft from } \\
\text { left bank) }\end{array}$ & $\begin{array}{l}\text { Speciflc } \\
\text { conduct- } \\
\text { ance } \\
(\mu \mathrm{S} / \mathrm{cm})\end{array}$ & $\begin{array}{l}\mathrm{pH} \\
\text { (stan- } \\
\text { dard } \\
\text { units) }\end{array}$ & $\begin{array}{c}\text { Temper- } \\
\text { ature, } \\
\text { water } \\
\left({ }^{\circ} \mathrm{C}\right)\end{array}$ & $\begin{array}{l}\text { Trans - } \\
\text { parency } \\
\text { (secchl } \\
\text { disk) } \\
\text { (ft) }\end{array}$ & $\begin{array}{c}\text { Dissolved } \\
\text { oxygen } \\
(\mathrm{mg} / \mathrm{L})\end{array}$ & $\begin{array}{c}\text { Dissolved } \\
\text { oxygen } \\
\text { (percent } \\
\text { satura- } \\
\text { tion) }\end{array}$ \\
\hline
\end{tabular}

$\begin{array}{ccccc}\text { September } & & & \\ 20 & 0629 & 0.4 & 800 & 679 \\ 20 & 0629 & 3.0 & 800 & 680 \\ 20 & 0630 & 5.1 & 800 & 680 \\ 20 & 0630 & 9.6 & 800 & 680 \\ 20 & 0631 & 15 & 800 & 680 \\ 20 & 0631 & 19 & 800 & 680 \\ 20 & 0632 & 25 & 800 & 680 \\ 20 & 0632 & 27 & 800 & 681 \\ 20 & 1418 & 1.2 & 300 & 673 \\ 20 & 1418 & 3.5 & 300 & 676 \\ 20 & 1419 & 5.1 & 300 & 674 \\ 20 & 1419 & 11 & 300 & 675 \\ 20 & 1420 & 15 & 300 & 672 \\ 20 & 1421 & 20 & 300 & 676 \\ 20 & 1411 & 1.1 & 600 & 679 \\ 20 & 1412 & 3.4 & 600 & 678 \\ 20 & 1412 & 5.0 & 600 & 673 \\ 20 & 1413 & 10 & 600 & 670 \\ 20 & 1413 & 15 & 600 & 674 \\ 20 & 1414 & 20 & 600 & 672 \\ 20 & 1415 & 24 & 600 & 674 \\ 20 & 1415 & 31 & 600 & 678 \\ 20 & 1416 & 35 & 600 & 670 \\ 20 & 1406 & 1.1 & 800 & 673 \\ 20 & 1406 & 3.0 & 800 & 673 \\ 20 & 1407 & 5.1 & 800 & 675 \\ 20 & 1407 & 10 & 800 & 673 \\ 20 & 1408 & 15 & 800 & 670 \\ 20 & 1408 & 20 & 800 & 671 \\ 20 & 1409 & 24 & 800 & 671 \\ 20 & 1409 & 27 & 800 & 685\end{array}$

$\begin{array}{ll}7.5 & 24.0 \\ 7.4 & 24.0 \\ 7.4 & 24.0 \\ 7.4 & 24.0 \\ 7.4 & 24.0 \\ 7.4 & 24.0 \\ 7.4 & 24.0 \\ 7.4 & 24.0 \\ 7.5 & 24.0 \\ 7.5 & 24.0 \\ 7.5 & 24.0 \\ 7.5 & 24.0 \\ 7.5 & 24.0 \\ 7.5 & 23.9 \\ 7.5 & 24.0 \\ 7.5 & 24.0 \\ 7.5 & 24.0 \\ 7.5 & 24.0 \\ 7.5 & 24.0 \\ 7.5 & 24.0 \\ 7.5 & 24.0 \\ 7.5 & 24.0 \\ 7.5 & 24.0 \\ 7.5 & 24.0 \\ 7.5 & 24.0 \\ 7.5 & 24.0 \\ 7.5 & 24.0 \\ 7.5 & 24.0 \\ 7.5 & 24.0 \\ 7.5 & 24.0 \\ 7.5 & 24.0\end{array}$

\begin{tabular}{|c|c|c|}
\hline$\cdots$ & 5.9 & 72 \\
\hline- & 5.9 & 72 \\
\hline- & 5.9 & 72 \\
\hline - - & 5.9 & 72 \\
\hline$\cdots$ & 5.9 & 72 \\
\hline$\cdots$ & 5.9 & 71 \\
\hline- & 5.9 & 71 \\
\hline$\cdots$ & 5.7 & 69 \\
\hline$\cdots$ & 6.1 & 73 \\
\hline- & 6.0 & 72 \\
\hline$\cdots$ & 6.0 & 72 \\
\hline- & 6.0 & 73 \\
\hline$-\cdot$ & 6.0 & 72 \\
\hline$\cdots$ & 6.0 & 72 \\
\hline- & 6.1 & 73 \\
\hline$\cdots$ & 6.0 & 72 \\
\hline$\cdots$ & 6.0 & 73 \\
\hline$\cdots$ & 6.0 & 72 \\
\hline-- & 6.1 & 73 \\
\hline$\cdots$ & 6.1 & 73 \\
\hline$\cdots$ & 6.1 & 73 \\
\hline$\cdots$ & 6.0 & 73 \\
\hline 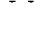 & 5.9 & \\
\hline$\cdots$ & 6.1 & 73 \\
\hline$\cdots$ & 6.0 & 72 \\
\hline$\cdots$ & 5.9 & 72 \\
\hline$\cdots$ & 6.0 & 72 \\
\hline$\cdots$ & 5.9 & 72 \\
\hline$\cdots$ & 5.9 & 71 \\
\hline$\cdots$ & 5.9 & 71 \\
\hline- & 5.9 & \\
\hline
\end{tabular}


Table 24. Water-quality data for station 391447081414201, Ohio River at river mile 192.9, June to October, 1995, Continued.

[Et = Eeet; $\mu \mathrm{S} / \mathrm{cm}=$ microsiemens per centımeter $;{ }^{\circ} \mathrm{C}=$ degrees Celsius:

$\mathrm{mg} / \mathrm{L}=\mathrm{mili}$ igrams per liter; - - = data not collected]

\begin{tabular}{|c|c|c|c|c|c|c|c|c|c|}
\hline Date & Time & $\begin{array}{l}\text { Sampling } \\
\text { depth } \\
\text { ( } f t)\end{array}$ & $\begin{array}{l}\text { Sample } \\
\text { location } \\
\text { (ft from } \\
\text { left bank) }\end{array}$ & $\begin{array}{l}\text { Specific } \\
\text { conduct - } \\
\text { ance } \\
(\mu \mathrm{S} / \mathrm{cm})\end{array}$ & $\begin{array}{l}\mathrm{pH} \\
\text { (stan- } \\
\text { dard } \\
\text { units) }\end{array}$ & $\begin{array}{c}\text { Temper. } \\
\text { ature, } \\
\text { water } \\
\left({ }^{\circ} \mathrm{C}\right)\end{array}$ & $\begin{array}{l}\text { Trans - } \\
\text { parency } \\
\text { (secchl } \\
\text { disk) } \\
\text { (ft) }\end{array}$ & $\begin{array}{l}\text { Dissolved } \\
\text { oxygen } \\
(\mathrm{mg} / \mathrm{L})\end{array}$ & $\begin{array}{c}\text { Dissolved } \\
\text { oxygen } \\
\text { (percent } \\
\text { satura- } \\
\text { tion) }\end{array}$ \\
\hline
\end{tabular}

\begin{tabular}{|c|c|c|c|}
\hline \multicolumn{4}{|c|}{ October } \\
\hline 05 & 0616 & 0.6 & 300 \\
\hline 05 & 0617 & 3.1 & 300 \\
\hline 05 & 0617 & 4.9 & 300 \\
\hline 05 & 0618 & 9.8 & 300 \\
\hline 05 & 0625 & 15 & 300 \\
\hline 05 & 0626 & 20 & 30 \\
\hline 05 & 0627 & 25 & 30 \\
\hline 05 & 0632 & 1.1 & 60 \\
\hline 05 & 0633 & 3.0 & 60 \\
\hline 05 & 0633 & 5.0 & 60 \\
\hline 05 & 0634 & 10 & \\
\hline 05 & 0635 & 15 & \\
\hline 05 & 0635 & 20 & \\
\hline 05 & 0636 & 25 & 60 \\
\hline 05 & 0637 & 30 & \\
\hline 05 & 0642 & 1.0 & \\
\hline 05 & 0642 & 3.0 & \\
\hline 05 & 0643 & 4.9 & \\
\hline 05 & 0643 & 10 & \\
\hline 05 & 0644 & 15 & \\
\hline 05 & 0644 & 20 & \\
\hline 05 & 0645 & 25 & \\
\hline 05 & 0645 & 29 & \\
\hline 05 & 1217 & 0.9 & 30 \\
\hline 05 & 1217 & 2.9 & 30 \\
\hline 05 & 1218 & 4.8 & 30 \\
\hline 05 & 1218 & 10 & 3 \\
\hline 05 & 1219 & 15 & 3 \\
\hline 05 & 1219 & 20 & 3 \\
\hline 05 & 1220 & 25 & \\
\hline 05 & & 28 & \\
\hline
\end{tabular}

620
624
627
630
627
638
623

626
626
628
633
631
639
643
637
630
627
638
634
635
638
643
646
633
636
639
636
639
640
640
640

$\begin{array}{ll}7.3 & 21.8 \\ 7.3 & 21.8 \\ 7.3 & 21.8 \\ 7.3 & 21.8 \\ 7.3 & 21.8 \\ 7.3 & 21.8 \\ 7.3 & 21.8 \\ 7.3 & 21.9 \\ 7.3 & 21.9 \\ 7.3 & 21.9 \\ 7.3 & 21.9 \\ 7.3 & 21.9 \\ 7.3 & 21.9 \\ 7.3 & 21.2 \\ 7.3 & 21.9 \\ 7.3 & 21.9 \\ 7.3 & 21.9 \\ 7.3 & 21.9 \\ 7.3 & 21.9 \\ 7.3 & 21.9 \\ 7.3 & 21.9 \\ 7.3 & 21.9 \\ 7.3 & 21.9 \\ 7.3 & \\ 7.4 & 22.1 \\ 7.4 & 22.1 \\ 7.3 & 22.0 \\ 7.3 & 21.9 \\ 7.3 & 21.9 \\ 7.3 & 21.9 \\ 7.3 & 21.9 \\ 7.3 & 21.9\end{array}$

$-7.2$

7.1

- 7.1

7.1

7.0

- 6.9

6.9

7.0

7.0
6.8

$\begin{array}{ll}\cdots & 6.8 \\ \cdots & 6.9\end{array}$

6.9

$-7.0$

6.9

6.8

6.2

84

627

623

- 6.8

- 6.9

- 6.8

6.8

6.7

6.7

$\begin{array}{llllll}643 & 7.3 & 21.9 & \ldots & 6.8 & 79\end{array}$

$\begin{array}{llllll}643 & 7.3 & 21.9 & \ldots & 6.8 & 79 \\ 646 & 7.3 & 21.9 & -- & 6.9 & 80\end{array}$

82

82

82

81

80
80

82

$\begin{array}{llll}635 & 7.3 & 21.9\end{array}$

$\begin{array}{llll}638 & 7.3 & 21.9 & -\end{array}$

$\begin{array}{llllll}633 & 7.4 & 22.1 & \ldots & 6.9 & 81\end{array}$

$\begin{array}{llllll}636 & 7.4 & 22.1 & -. & 6.9 & 81\end{array}$

$\begin{array}{llllll}639 & 7.3 & 22.0 & - & 6.9 & 80\end{array}$

$\begin{array}{llllll}636 & 7.3 & 21.9 & - & 6.7 & 78\end{array}$

$\begin{array}{llllll}639 & 7.3 & 21.9 & - & 6.7 & 78\end{array}$

$\begin{array}{llll}21.9 & \cdots & 6.7 & 78 \\ 21.9 & \cdots & 6.7 & 78\end{array}$

$\begin{array}{lllll}640 & 7.3 & 21.9 & - & 6.7\end{array}$ 
Table 24. Water-quality data for station 391447081414201, Ohio River at river mile 192.9, June to October, 1995, Continued.

ft $=$ feet; $\mu \mathrm{S} / \mathrm{cm}=$ microsiemens per centimeter; ${ }^{\circ} \mathrm{C}=$ degrees Celsius;

$\mathrm{mg} / \mathrm{L}=\mathrm{milligrams}$ per liter; $\cdots$ = data not collected]

\begin{tabular}{|c|c|c|c|c|c|c|c|c|c|}
\hline Date & Time & $\begin{array}{l}\text { Sampling } \\
\text { depth } \\
(E t)\end{array}$ & $\begin{array}{c}\text { Sample } \\
\text { location } \\
\text { (ft from } \\
\text { left bank) }\end{array}$ & $\begin{array}{l}\text { Specific } \\
\text { conduct- } \\
\text { ance } \\
(\mu \mathrm{s} / \mathrm{cm})\end{array}$ & $\begin{array}{l}\mathrm{pH} \\
\text { (stan- } \\
\text { dard } \\
\text { units) }\end{array}$ & $\begin{array}{c}\text { Temper- } \\
\text { ature, } \\
\text { water } \\
\left({ }^{\circ} \mathrm{C}\right)\end{array}$ & $\begin{array}{c}\text { Trans - } \\
\text { parency } \\
\text { (Secchi } \\
\text { dlsk) } \\
(\mathrm{Et})\end{array}$ & $\begin{array}{c}\text { Dissolved } \\
\text { oxygen } \\
\text { (mg/L) }\end{array}$ & $\begin{array}{c}\text { Dissolved } \\
\text { oxygen } \\
\text { (percent } \\
\text { satura- } \\
\text { tion) }\end{array}$ \\
\hline
\end{tabular}

\begin{tabular}{|c|c|c|c|c|c|c|c|c|c|}
\hline \multicolumn{10}{|c|}{ October } \\
\hline 05 & 1206 & 1.1 & 600 & 631 & 7.4 & 22.0 & $\cdots$ & 6.9 & 80 \\
\hline 05 & 1205 & 3.1 & 600 & 636 & 7.4 & 22.0 & - & 6.9 & 80 \\
\hline 05 & 1205 & 5.0 & 600 & 632 & 7.3 & 21.9 & 3.5 & 6.8 & 79 \\
\hline 05 & 1204 & 9.8 & 600 & 638 & 7.3 & 21.9 & - - & 6.8 & 79 \\
\hline 05 & 1203 & 15 & 600 & 639 & 7.3 & 21.8 & - & 6.8 & 79 \\
\hline 05 & 1203 & 21 & 600 & 635 & 7.3 & 21.8 & -- & 6.7 & 78 \\
\hline 05 & 1202 & 26 & 600 & 639 & 7.3 & 21.8 & $\cdots$ & 6.8 & 79 \\
\hline 05 & 1202 & 28 & 600 & 635 & 7.3 & 21.8 & -- & 6.7 & 78 \\
\hline 05 & 1209 & 0.4 & 800 & 629 & 7.3 & 22.0 & $-\cdot$ & 7.0 & 81 \\
\hline 05 & 1209 & 2.9 & 800 & 631 & 7.4 & 22.0 & - - & 7.0 & 81 \\
\hline 05 & 1210 & 4.9 & 800 & 633 & 7.4 & 21.9 & -- & 7.0 & 81 \\
\hline 05 & 1210 & 10 & 800 & 635 & 7.4 & 21.9 & - & 6.9 & 80 \\
\hline 05 & 1211 & 16 & 800 & 638 & 7.4 & 21.9 & $\cdots$ & 6.9 & U \\
\hline 05 & 1211 & 20 & 800 & 636 & 7.4 & 21.8 & $-\cdot$ & 6.8 & \\
\hline 05 & 1212 & 25 & 800 & 639 & 7.4 & 21.8 & $\cdots$ & 6.9 & 80 \\
\hline 19 & 1525 & 0.9 & 300 & 541 & 7.6 & 19.3 & $\cdots$ & 8.1 & 89 \\
\hline 19 & 1525 & 3.1 & 300 & 537 & 7.6 & 19.2 & $\cdots$ & 8.1 & \\
\hline 19 & 1526 & 5.0 & 300 & 540 & 7.6 & 19.0 & -- & 8.0 & 87 \\
\hline 19 & 1526 & 9.7 & 300 & 538 & 7.5 & 18.6 & -- & 7.6 & 83 \\
\hline 19 & 1527 & 15 & 300 & 541 & 7.5 & 18.5 & - & 7.6 & 82 \\
\hline 19 & 1527 & 20 & 300 & 545 & 7.5 & 18.5 & - & 7.5 & 8 \\
\hline 19 & 1528 & 25 & 300 & 541 & 7.5 & 18.5 & $\cdots$ & 7.5 & \\
\hline 19 & 1528 & 29 & 300 & 544 & 7.5 & 18.5 & -- & 7.4 & 0 \\
\hline 19 & 1530 & 1.0 & 600 & 540 & 7.6 & 19.1 & $\cdots$ & 8.0 & 88 \\
\hline 19 & 1531 & 3.1 & 600 & 537 & 7.6 & 19.1 & 2.0 & 8.1 & 8 \\
\hline 19 & 1531 & 5.1 & 600 & 539 & 7.5 & 19.1 & -- & 8.0 & 8 \\
\hline 19 & 1532 & 9.9 & 600 & 536 & 7.5 & 18.6 & $\cdots$ & 7.7 & 8 \\
\hline 19 & 1532 & 15 & 600 & 539 & 7.5 & 18.6 & $\cdots$ & 7.5 & 8 \\
\hline 19 & 1533 & 20 & 600 & 541 & 7.5 & 18.6 & $\cdots$ & 7.5 & 8 \\
\hline 19 & 1533 & 25 & 600 & 536 & 7.5 & 18.6 & $\cdots$ & 7.5 & 8 \\
\hline 19 & 1534 & 29 & 600 & 531 & 7.5 & 18.6 & - & 7.5 & \\
\hline
\end{tabular}


Table 24. Water-quality data for station 391447081414201, Ohio River at river mile 192.9, June to October, 1995, Continued.

[ft $=$ feet; $\mu \mathrm{S} / \mathrm{cm}=$ microsiemens per centimeter; ${ }^{\circ} \mathrm{C}=$ degrees Celsius:

$\mathrm{mg} / \mathrm{L}=\mathrm{milligrams}$ per liter; $\cdots$ = data not collected]

\begin{tabular}{|c|c|c|c|c|c|c|c|c|c|}
\hline Date & Time & $\begin{array}{l}\text { Sampling } \\
\text { depth } \\
\text { (Et) }\end{array}$ & $\begin{array}{c}\text { Sample } \\
\text { location } \\
\text { (ft from } \\
\text { left bank) }\end{array}$ & $\begin{array}{l}\text { Specific } \\
\text { conduct } \\
\text { ance } \\
(\mu S / \mathrm{cm})\end{array}$ & $\begin{array}{l}\mathrm{pH} \\
\text { (stan- } \\
\text { dard } \\
\text { units) }\end{array}$ & $\begin{array}{c}\text { Temper- } \\
\text { ature, } \\
\text { water } \\
\left({ }^{\circ} \mathrm{C}\right)\end{array}$ & $\begin{array}{l}\text { Trans- } \\
\text { parency } \\
\text { (secchi } \\
\text { disk) } \\
(t t)\end{array}$ & $\begin{array}{c}\text { Dissolved } \\
\text { oxygen } \\
\text { (mg/L) }\end{array}$ & $\begin{array}{c}\text { Dissolved } \\
\text { oxygen } \\
\text { (percent } \\
\text { satura- } \\
\text { tion) }\end{array}$ \\
\hline
\end{tabular}

October

\begin{tabular}{rlllllllll}
19 & 1536 & 0.9 & 800 & 541 & 7.6 & 19.3 & $\ldots$ & 8.1 & 89 \\
19 & 1536 & 3.1 & 800 & 538 & 7.5 & 19.0 & $\ldots$ & 8.0 & 87 \\
19 & 1537 & 5.1 & 800 & 536 & 7.5 & 19.0 & $\ldots$ & 7.9 & 86 \\
19 & 1537 & 10 & 800 & 536 & 7.5 & 18.6 & $\ldots$ & 7.7 & 83 \\
19 & 1538 & 15 & 800 & 538 & 7.5 & 18.6 & $\ldots$ & 7.6 & 82 \\
19 & 1538 & 20 & 800 & 546 & 7.5 & 18.6 & $\ldots$ & 7.5 & 81 \\
19 & 1539 & 25 & 800 & 546 & 7.5 & 18.6 & $\ldots$ & 7.5 & 81 \\
\hline
\end{tabular}


Table 25. Water-quality data for station 391351081412201, Ohio River at river mile 194.0, June to October, 1995.

[Et $=$ Eeet $; \mu \mathrm{S} / \mathrm{cm}=$ microsiemens per centimeter $;{ }^{\circ} \mathrm{C}=$ degrees Celsius:

$\mathrm{mg} / \mathrm{L}=$ milligrams per liter; $\cdots$ = data not collected]

\begin{tabular}{|c|c|c|c|c|c|c|c|c|c|}
\hline Date & Time & $\begin{array}{l}\text { Sampling } \\
\text { depth } \\
(\mathrm{f} t)\end{array}$ & $\begin{array}{l}\text { Sample } \\
\text { location } \\
\text { (ft from } \\
\text { left bank) }\end{array}$ & $\begin{array}{l}\text { Specific } \\
\text { conduct- } \\
\text { ance } \\
(\mu \mathrm{s} / \mathrm{cm})\end{array}$ & $\begin{array}{l}\mathrm{pH} \\
\text { (stan- } \\
\text { dard } \\
\text { units) }\end{array}$ & $\begin{array}{c}\text { Temper- } \\
\text { ature, } \\
\text { water } \\
\left({ }^{\circ} \mathrm{C}\right)\end{array}$ & $\begin{array}{l}\text { Trans - } \\
\text { parency } \\
\text { (Secchl } \\
\text { disk) } \\
(\mathrm{Et})\end{array}$ & $\begin{array}{l}\text { Dissolved } \\
\text { oxygen } \\
(\mathrm{mg} / \mathrm{L})\end{array}$ & $\begin{array}{c}\text { Dissolved } \\
\text { oxygen } \\
\text { (percent } \\
\text { satura- } \\
\text { tion) }\end{array}$ \\
\hline
\end{tabular}

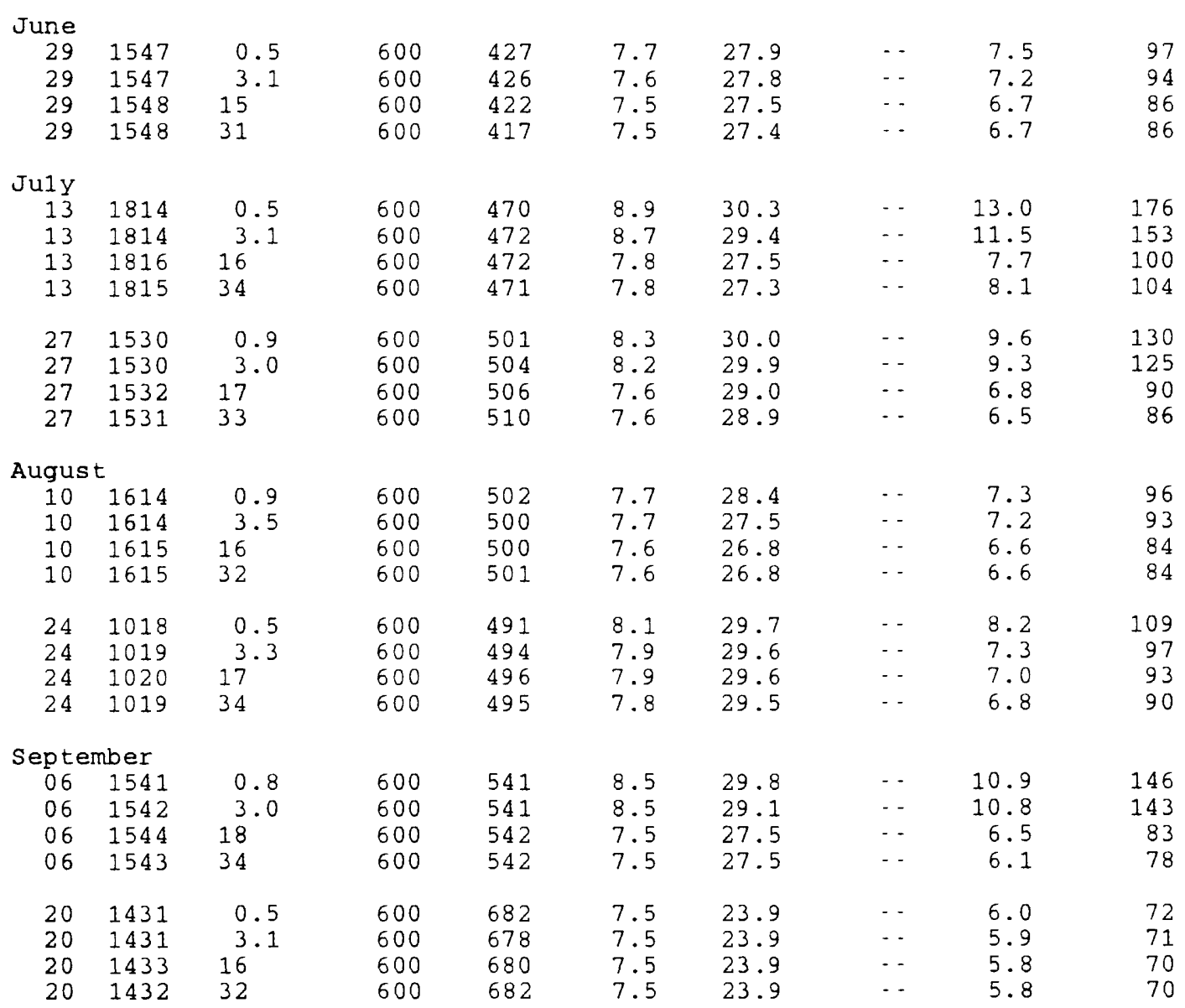


Table 25. Water-quality data for station 391351081412201, Ohio River at river mile 194.0, June to October, 1995, Continued.

[Et $=$ feet $; \mu \mathrm{S} / \mathrm{cm}=$ microsiemens per centimeter: ${ }^{\circ} \mathrm{C}=$ degrees Celsius:

$\mathrm{mg} / \mathrm{L}=$ milligrams per liter;. = data not collected]

\begin{tabular}{|c|c|c|c|c|c|c|c|c|c|}
\hline Date & Time & $\begin{array}{l}\text { Sampling } \\
\text { depth } \\
( \pm t)\end{array}$ & $\begin{array}{c}\text { Sample } \\
\text { location } \\
\text { (ft Erom } \\
\text { left bank) }\end{array}$ & $\begin{array}{l}\text { Specific } \\
\text { conduct- } \\
\text { ance } \\
(\mu \mathrm{S} / \mathrm{cm})\end{array}$ & $\begin{array}{l}\text { pH } \\
\text { (stan- } \\
\text { dard } \\
\text { units) }\end{array}$ & $\begin{array}{c}\text { Temper- } \\
\text { ature, } \\
\text { water } \\
\left({ }^{\circ} \mathrm{C}\right)\end{array}$ & $\begin{array}{l}\text { Trans- } \\
\text { parency } \\
\text { (Secchl } \\
\text { disk) } \\
\text { (tt) }\end{array}$ & $\begin{array}{l}\text { Dissolved } \\
\text { oxygen } \\
(\mathrm{mg} / \mathrm{L})\end{array}$ & $\begin{array}{c}\text { Dissolved } \\
\text { oxygen } \\
\text { (percent } \\
\text { satura- } \\
\text { tion) }\end{array}$ \\
\hline \multicolumn{10}{|c|}{ October } \\
\hline 05 & 1228 & 0.5 & 600 & 631 & 7.4 & 22.1 & $\cdots$ & 7.1 & 82 \\
\hline 05 & 1228 & 3.1 & 600 & 636 & 7.4 & 22.0 & -- & 7.0 & 81 \\
\hline 05 & 1230 & 16 & 600 & 640 & 7.3 & 21.8 & . & 6.9 & 79 \\
\hline 05 & 1229 & 32 & 600 & 640 & 7.3 & 21.8 & $\cdots$ & 6.8 & 79 \\
\hline 19 & 1600 & 0.9 & 600 & 538 & 7.5 & 18.6 & - & 7.5 & 81 \\
\hline 19 & 1601 & 3.0 & 600 & 538 & 7.5 & 18.8 & - & 7.7 & 84 \\
\hline 19 & 1603 & 17 & 600 & 542 & 7.5 & 18.7 & $\ldots$ & 7.6 & 82 \\
\hline 19 & 1602 & 33 & 600 & 540 & 7.5 & 18.6 & -- & 7.5 & 80 \\
\hline
\end{tabular}


Table 26. Water-quality data for station 391302081425101, Ohio River at river mile 195.8, June to October, 1995.

\begin{tabular}{|c|c|c|c|c|c|c|c|c|c|}
\hline Date & Time & $\begin{array}{l}\text { Sampling } \\
\text { depth } \\
\text { (ft) }\end{array}$ & $\begin{array}{c}\text { Sample } \\
\text { location } \\
\text { (ft from } \\
\text { left bank) }\end{array}$ & $\begin{array}{l}\text { Specific } \\
\text { conduct- } \\
\text { ance } \\
(\mu \mathrm{s} / \mathrm{cm})\end{array}$ & $\begin{array}{l}\mathrm{pH} \\
\text { (stan- } \\
\text { dard } \\
\text { units) }\end{array}$ & $\begin{array}{c}\text { Temper- } \\
\text { ature, } \\
\text { water } \\
\left({ }^{\circ} \mathrm{C}\right)\end{array}$ & $\begin{array}{c}\text { Trans- } \\
\text { parency } \\
\text { (SecchI } \\
\text { disk) } \\
\text { (ft) }\end{array}$ & $\begin{array}{l}\text { Dissolved } \\
\text { oxygen } \\
(\mathrm{mg} / \mathrm{L})\end{array}$ & $\begin{array}{c}\text { Dissolved } \\
\text { oxygen } \\
\text { (percent } \\
\text { satura- } \\
\text { tion) }\end{array}$ \\
\hline \multicolumn{10}{|l|}{ June } \\
\hline 29 & 1552 & 0.3 & 600 & 407 & 7.5 & 28.0 & -- & 7.2 & 93 \\
\hline 29 & 1552 & 2.6 & 600 & 409 & 7.5 & 27.7 & -- & 6.9 & 89 \\
\hline 29 & 1553 & 19 & 600 & 418 & 7.4 & 27.4 & -- & 6.5 & 84 \\
\hline 29 & 1553 & 38 & 600 & 418 & 7.4 & 27.3 & $\cdots$ & 6.5 & 83 \\
\hline \multicolumn{10}{|l|}{ July } \\
\hline 13 & 1820 & 0.5 & 600 & 465 & 8.8 & 29.9 & $\cdots$ & 12.7 & 170 \\
\hline 13 & 1820 & 3.4 & 600 & 467 & 8.7 & 29.2 & $-\cdot$ & 12.4 & 164 \\
\hline 13 & 1822 & 19 & 600 & 472 & 7.7 & 27.4 & $\cdots$ & 7.5 & 97 \\
\hline 13 & 1822 & 38 & 600 & 471 & 7.7 & 27.3 & $\cdots$ & 7.3 & 93 \\
\hline 27 & 1547 & 0.4 & 600 & 498 & 7.7 & 29.4 & $\cdots$ & 7.7 & 102 \\
\hline 27 & 1547 & 3.0 & 600 & 495 & 7.7 & 29.4 & - & 7.7 & 102 \\
\hline 27 & 1549 & 18 & 600 & 495 & 7.5 & 29.1 & $\cdots$ & 6.7 & 88 \\
\hline 27 & 1548 & 37 & 600 & 494 & 7.5 & 29.0 & $\cdots$ & 6.6 & 87 \\
\hline \multicolumn{10}{|c|}{ August } \\
\hline 10 & 1619 & 1.3 & 600 & 503 & 7.7 & 27.9 & - & 7.1 & 92 \\
\hline 10 & 1619 & 3.4 & 600 & 504 & 7.7 & 27.9 & $\cdots$ & 7.0 & 91 \\
\hline 10 & 1621 & 18 & 600 & 502 & 7.6 & 27.1 & $\cdots$ & 6.3 & 81 \\
\hline 10 & 1620 & 37 & 600 & 502 & 7.6 & 27.0 & - & 6.3 & 81 \\
\hline 24 & 1013 & 0.4 & 600 & 489 & 8.0 & 29.8 & $\cdots$ & 7.9 & 105 \\
\hline 24 & 1013 & 3.4 & 600 & 489 & 7.9 & 29.7 & $\cdots$ & 7.2 & 97 \\
\hline 24 & 1014 & 19 & 600 & 488 & 7.8 & 29.7 & $\cdots$ & 6.7 & 90 \\
\hline 24 & 1014 & 38 & 600 & 489 & 7.8 & 29.6 & $\cdots$ & 6.6 & 88 \\
\hline \multicolumn{10}{|c|}{ September } \\
\hline 06 & 1550 & 0.5 & 600 & 540 & 8.2 & 29.8 & - & 9.6 & 128 \\
\hline 06 & 1550 & 2.9 & 600 & 538 & 8.2 & 28.9 & $\cdots$ & 9.6 & 126 \\
\hline 06 & 1552 & 19 & 600 & 538 & 7.4 & 27.5 & - & 6.1 & 79 \\
\hline 06 & 1551 & 39 & 600 & 543 & 7.4 & 27.4 & $\cdots$ & 5.8 & 74 \\
\hline 20 & 1439 & 0.9 & 600 & 688 & 7.5 & 24.0 & $\cdots$ & 5.7 & 68 \\
\hline 20 & 1439 & 2.9 & 600 & 681 & 7.4 & 24.1 & $\cdots$ & 5.7 & 68 \\
\hline 20 & 1441 & 21 & 600 & 684 & 7.4 & 24.1 & - & 5.6 & 68 \\
\hline 20 & 1440 & 40 & 600 & 683 & 7.4 & 24.0 & $\cdots$ & 5.6 & 68 \\
\hline
\end{tabular}


Table 26. Water-quality data for station 391302081425101, Ohio River at river mile 195.8, June to October, 1995, Continued.

$\left[\mathrm{ft}=\mathrm{feet} ; \mu \mathrm{S} / \mathrm{cm}=\right.$ microsiemens per centımeter: ${ }^{\circ} \mathrm{C}=$ degrees Celsius;

$\mathrm{mg} / \mathrm{L}=\mathrm{mili}$ igrams per liter: $\cdot$ = data not collected]

\begin{tabular}{|c|c|c|c|c|c|c|c|c|c|}
\hline Date & Time & $\begin{array}{l}\text { Sampling } \\
\text { depth } \\
\text { (ft) }\end{array}$ & $\begin{array}{c}\text { Sample } \\
\text { location } \\
\text { (ft from } \\
\text { left bank) }\end{array}$ & $\begin{array}{l}\text { Specitic } \\
\text { conduct. } \\
\text { ance } \\
(\mu \mathrm{S} / \mathrm{cm})\end{array}$ & $\begin{array}{l}\text { pH } \\
\text { (stan- } \\
\text { dard } \\
\text { unzts) }\end{array}$ & $\begin{array}{c}\text { Temper- } \\
\text { ature, } \\
\text { water } \\
\left({ }^{\circ} \mathrm{C}\right)\end{array}$ & $\begin{array}{l}\text { Trans - } \\
\text { parency } \\
\text { (Secchi } \\
\text { disk) } \\
(\mathrm{ft})\end{array}$ & $\begin{array}{l}\text { Dissolved } \\
\text { oxygen } \\
\text { (mg/L) }\end{array}$ & $\begin{array}{c}\text { Dissolved } \\
\text { oxygen } \\
\text { (percent } \\
\text { satura- } \\
\text { tion) }\end{array}$ \\
\hline \multicolumn{10}{|c|}{ October } \\
\hline 05 & 1237 & 0.6 & 600 & 624 & 7.3 & 21.9 & $\cdots$ & 7.0 & 81 \\
\hline 05 & 1238 & 3.0 & 600 & 621 & 7.3 & 21.9 & $\cdots$ & 7.0 & 81 \\
\hline 05 & 1236 & 19 & 600 & 635 & 7.3 & 21.8 & $-\cdot$ & 6.9 & 80 \\
\hline 05 & 1236 & 35 & 600 & 631 & 7.3 & 21.8 & $\cdots$ & 6.8 & 79 \\
\hline 19 & 1608 & 0.4 & 600 & 537 & 7.6 & 19.2 & $\cdots$ & 8.0 & 88 \\
\hline 19 & 1608 & 3.2 & 600 & 536 & 7.5 & 18.7 & $-\cdot$ & 7.6 & 83 \\
\hline 19 & 1610 & 19 & 600 & 535 & 7.5 & 18.5 & $-\cdot$ & 7.4 & 80 \\
\hline 19 & 1609 & 37 & 600 & 532 & 7.4 & 18.4 & $\cdots$ & 7.4 & 79 \\
\hline
\end{tabular}


Table 27. Water-quality data for station 391146081440501, Ohio River at river mile 197.9, June to October, 1995.

$\mathrm{ft}=\mathrm{feet} ; \mu \mathrm{S} / \mathrm{cm}=$ microsiemens per centimeter $;{ }^{\circ} \mathrm{C}=$ degrees Celsius $\mathrm{mg} / \mathrm{L}=$ miligrams per liter: . = data not collected]

\begin{tabular}{|c|c|c|c|c|c|c|c|c|c|}
\hline Date & Time & $\begin{array}{l}\text { Sampling } \\
\text { depth } \\
\text { (ft) }\end{array}$ & $\begin{array}{l}\text { Sample } \\
\text { location } \\
\text { (Et from } \\
\text { left bank) }\end{array}$ & $\begin{array}{l}\text { Specific } \\
\text { conduct- } \\
\text { ance } \\
(\mu \mathrm{S} / \mathrm{cm})\end{array}$ & $\begin{array}{l}\mathrm{pH} \\
\text { (stan- } \\
\text { dard } \\
\text { units) }\end{array}$ & $\begin{array}{c}\text { Temper- } \\
\text { ature, } \\
\text { water } \\
\left({ }^{\circ} \mathrm{C}\right)\end{array}$ & $\begin{array}{c}\text { Trans- } \\
\text { parency } \\
\text { (Secchi } \\
\text { disk) } \\
(\mathrm{ft})\end{array}$ & $\begin{array}{l}\text { Dissolved } \\
\text { oxygen } \\
\text { (mg/L) }\end{array}$ & $\begin{array}{c}\text { Dissolved } \\
\text { oxygen } \\
\text { (percent } \\
\text { satura- } \\
\text { tion) }\end{array}$ \\
\hline
\end{tabular}

\begin{tabular}{|c|c|c|c|c|c|c|c|c|c|}
\hline \multicolumn{10}{|l|}{ June } \\
\hline 29 & 1557 & 0.5 & 500 & 404 & 7.5 & 27.6 & - & 6.8 & 88 \\
\hline 29 & 1557 & 3.0 & 500 & 403 & 7.4 & 27.6 & $\cdots$ & 6.7 & 87 \\
\hline 29 & 1559 & 16 & 500 & 410 & 7.4 & 27.3 & - & 6.4 & 82 \\
\hline 29 & 1558 & 31 & 500 & 409 & 7.4 & 27.3 & $\cdots$ & 6.4 & 83 \\
\hline \multicolumn{10}{|l|}{ July } \\
\hline 13 & 1826 & 0.5 & 500 & 461 & 8.9 & 30.2 & $\cdots$ & 13.2 & 179 \\
\hline 13 & 1827 & 2.9 & 500 & 463 & 8.7 & 29.2 & - & 11.7 & 155 \\
\hline 13 & 1829 & 15 & 500 & 467 & 7.9 & 27.6 & $\cdots$ & 8.1 & 105 \\
\hline 13 & 1828 & 29 & 500 & 467 & 7.8 & 27.3 & $\cdots$ & 7.5 & 97 \\
\hline 27 & 1555 & 0.5 & 500 & 495 & 7.7 & 29.5 & $\cdots$ & 7.5 & 100 \\
\hline 27 & 1555 & 3.0 & 500 & 495 & 7.7 & 29.5 & $\cdots$ & 7.5 & 100 \\
\hline 27 & 1557 & 15 & 500 & 495 & 7.5 & 29.1 & $\cdots$ & 6.8 & 90 \\
\hline 27 & 1556 & 31 & 500 & 495 & 7.5 & 29.0 & - & 6.6 & 88 \\
\hline \multicolumn{10}{|c|}{ August } \\
\hline 10 & 1624 & 1.1 & 500 & 488 & 7.7 & 28.1 & $\cdots$ & 6.9 & 90 \\
\hline 10 & 1624 & 3.4 & 500 & 490 & 7.6 & 27.9 & $\cdots$ & 6.8 & 88 \\
\hline 10 & 1625 & 15 & 500 & 498 & 7.6 & 27.1 & - - & 6.2 & 79 \\
\hline 10 & 1625 & 30 & 500 & 498 & 7.6 & 27.1 & $\cdots$ & 6.3 & 80 \\
\hline 24 & 1008 & 0.5 & 500 & 485 & 8.0 & 29.8 & -- & 7.6 & 102 \\
\hline 24 & 1008 & 3.4 & 500 & 487 & 7.9 & 29.8 & $\cdots$ & 7.2 & 96 \\
\hline 24 & 1009 & 17 & 500 & 487 & 7.8 & 29.6 & $\cdots$ & 6.6 & 88 \\
\hline 24 & 1009 & 34 & 500 & 490 & 7.8 & 29.6 & $\cdots$ & 6.5 & 87 \\
\hline \multicolumn{10}{|c|}{ September } \\
\hline 06 & 1558 & 0.2 & 500 & 536 & 8.2 & 29.7 & $\cdots$ & 9.8 & 130 \\
\hline 06 & 1558 & 2.8 & 500 & 537 & 8.2 & 29.6 & $\cdots$ & 9.2 & 122 \\
\hline 06 & 1600 & 20 & 500 & 535 & 7.5 & 27.5 & $\cdots$ & 5.9 & 76 \\
\hline 06 & 1559 & 41 & 500 & 538 & 7.5 & 27.5 & $\cdots$ & 5.6 & 72 \\
\hline 20 & 1445 & 0.5 & 500 & 681 & 7.4 & 24.0 & $\cdots$ & 5.6 & 68 \\
\hline 20 & 1446 & 2.3 & 500 & 685 & 7.4 & 24.0 & - & 5.6 & 68 \\
\hline 20 & 1448 & 13 & 500 & 684 & 7.4 & 24.0 & $\cdots$ & 5.6 & 67 \\
\hline 20 & 1447 & 26 & 500 & 678 & 7.4 & 24.0 & - & 5.5 & 67 \\
\hline
\end{tabular}


Table 27. Water-quality data for station 391146081440501, Ohio River at river mile 197.9, June to October, 1995, Continued.

\begin{tabular}{|c|c|c|c|c|c|c|c|c|c|}
\hline Date & Time & $\begin{array}{l}\text { Sampling } \\
\text { depth } \\
\text { (ft) }\end{array}$ & $\begin{array}{c}\text { Sample } \\
\text { location } \\
\text { (ft from } \\
\text { left bank) }\end{array}$ & $\begin{array}{l}\text { Specific } \\
\text { conduct- } \\
\text { ance } \\
(\mu \mathrm{S} / \mathrm{cm})\end{array}$ & $\begin{array}{l}\mathrm{pH} \\
\text { (stan- } \\
\text { dard } \\
\text { units) }\end{array}$ & $\begin{array}{c}\text { Temper- } \\
\text { ature, } \\
\text { water } \\
\left({ }^{\circ} \mathrm{C}\right)\end{array}$ & $\begin{array}{l}\text { Trans - } \\
\text { parency } \\
\text { (Secchi } \\
\text { disk) } \\
(\mathrm{Et})\end{array}$ & $\begin{array}{l}\text { Dissolved } \\
\text { oxygen } \\
(\mathrm{mg} / \mathrm{L})\end{array}$ & $\begin{array}{c}\text { Dissolved } \\
\text { oxygen } \\
\text { (percent } \\
\text { satura- } \\
\text { tron) }\end{array}$ \\
\hline \multicolumn{10}{|c|}{ October } \\
\hline 05 & 1242 & 0.4 & 500 & 621 & 7.3 & 21.8 & - & 6.9 & 80 \\
\hline 05 & 1242 & 2.8 & 500 & 623 & 7.3 & 21.8 & $\cdots$ & 6.9 & 80 \\
\hline 05 & 1243 & 15 & 500 & 632 & 7.3 & 21.8 & $\cdots$ & 6.8 & 79 \\
\hline 05 & 1243 & 31 & 500 & 635 & 7.3 & 21.8 & $\cdots$ & 6.8 & 78 \\
\hline 19 & 1613 & 0.5 & 500 & 525 & 7.5 & 18.8 & $\cdots$ & 7.8 & 85 \\
\hline 19 & 1614 & 3.1 & 500 & 525 & 7.5 & 18.8 & $\ldots$ & 7.6 & 83 \\
\hline 19 & 1615 & 15 & 500 & 524 & 7.5 & 18.9 & - & 7.6 & 83 \\
\hline 19 & 1614 & 30 & 500 & 523 & 7.4 & 18.3 & $\cdots$ & 7.3 & 78 \\
\hline
\end{tabular}


Table 28. Water-quality data for station 391049081451601, Ohio River at river mile 199.5, June to October, 1995.

$\left[\mathrm{ft}=\mathrm{feet} ; \mu \mathrm{S} / \mathrm{cm}=\right.$ microsıemens per centimeter; ${ }^{\circ} \mathrm{C}=$ degrees celsius;

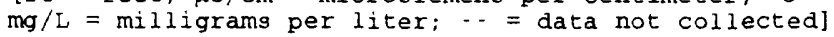

\begin{tabular}{|c|c|c|c|c|c|c|c|c|c|}
\hline Date & Time & $\begin{array}{l}\text { Sampling } \\
\text { depth } \\
\text { (ft) }\end{array}$ & $\begin{array}{l}\text { Sample } \\
\text { location } \\
\text { (ft from } \\
\text { left bank) }\end{array}$ & $\begin{array}{l}\text { Specific } \\
\text { conduct- } \\
\text { ance } \\
(\mu \mathrm{S} / \mathrm{cm})\end{array}$ & $\begin{array}{l}\mathrm{pH} \\
\text { (stan- } \\
\text { dard } \\
\text { units) }\end{array}$ & $\begin{array}{c}\text { Temper- } \\
\text { ature, } \\
\text { water } \\
\left({ }^{\circ} \mathrm{C}\right)\end{array}$ & $\begin{array}{c}\text { Trans- } \\
\text { parency } \\
\text { (secchi } \\
\text { disk) } \\
\text { (ft) }\end{array}$ & $\begin{array}{l}\text { Dissolved } \\
\text { oxygen } \\
(\mathrm{mg} / \mathrm{L})\end{array}$ & $\begin{array}{c}\text { Dissolved } \\
\text { oxygen } \\
\text { (percent } \\
\text { satura- } \\
\text { tion) }\end{array}$ \\
\hline
\end{tabular}

June

$\begin{array}{lllll}29 & 1603 & 0.4 & 500 & 393\end{array}$

$29 \quad 1603 \quad 3.0 \quad 500$

$29 \begin{array}{llll}29 & 1605 & 21 & 500 \\ 29 & 1604 & 39 & 500\end{array}$

$\begin{array}{llll}29 & 1604 & 39 & 500\end{array}$

393
395
399
396

$7.4 \quad 27.6$

$7.4 \quad 27.4$

$\begin{array}{ll}7.3 & 27.2 \\ 7.3 & 27.2\end{array}$

$-\cdot$

6.6

85

396

$\cdots$

6.4

6.2

80

July

$\begin{array}{llll}13 & 1847 & 0.5 & 500\end{array}$

$\begin{array}{llll}13 & 1847 & 3.1 & 500\end{array}$

465

465

473

8.8

29.3

$\begin{array}{ll}8.7 & 28.8\end{array}$

$\begin{array}{ll}7.7 & 27.2 \\ 7.7 & 26.8\end{array}$

- 12.3

- $\quad 11.7$

11.7

7.2
$-\quad 7.2$

154

$\begin{array}{llll}13 & 1848 & 43 & 500\end{array}$

515

$\begin{array}{ll}7.7 & 26.8\end{array}$

493

$\begin{array}{ll}7.8 & 29.3\end{array}$

$7.6 \quad 29.2$

$7.5 \quad 28.9$

-

7.8

- 6.8

-. 6.3

-. 6.2

92
91

494

$\begin{array}{ll}7.5 & 28.8\end{array}$

104

$\begin{array}{ll}500 & 494 \\ 500 & 506\end{array}$

28.8

Augus t

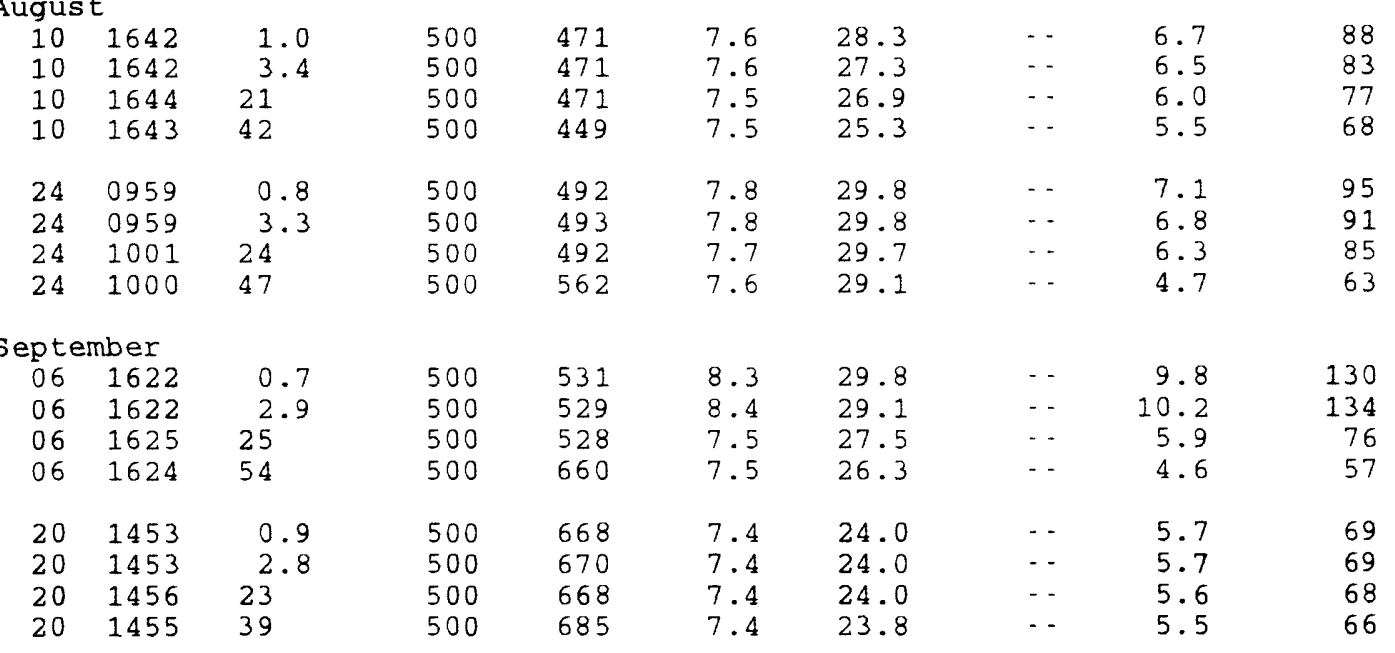


Table 28. Water-quality data for station 391049081451601, Ohio River at river mile 199.5, June to October, 1995, Continued.

[ft $=$ feet $; \mu \mathrm{s} / \mathrm{cm}=$ microsiemens per centımeter $;{ }^{\circ} \mathrm{C}=$ degrees Celsius; $\mathrm{mg} / \mathrm{L}=\mathrm{milligrams}$ per liter: -. = data not collected]

\begin{tabular}{|c|c|c|c|c|c|c|c|c|c|}
\hline Date & Time & $\begin{array}{l}\text { Sampling } \\
\text { depth } \\
( \pm t)\end{array}$ & $\begin{array}{c}\text { Sample } \\
\text { location } \\
\text { (ft from } \\
\text { left bank) }\end{array}$ & $\begin{array}{l}\text { Specific } \\
\text { conduct- } \\
\text { ance } \\
(\mu \mathrm{S} / \mathrm{cm})\end{array}$ & $\begin{array}{l}\text { pH } \\
\text { (stan- } \\
\text { dard } \\
\text { units) }\end{array}$ & $\begin{array}{c}\text { Temper- } \\
\text { ature, } \\
\text { water } \\
\left({ }^{\circ} \mathrm{C}\right)\end{array}$ & $\begin{array}{l}\text { Trans } \\
\text { parency } \\
\text { (Secchl } \\
\text { disk) } \\
\text { (ft) }\end{array}$ & $\begin{array}{l}\text { Dissolved } \\
\text { oxygen } \\
(\mathrm{mg} / \mathrm{L})\end{array}$ & $\begin{array}{c}\text { Dissolved } \\
\text { oxygen } \\
\text { (percent } \\
\text { satura- } \\
\text { tion) }\end{array}$ \\
\hline \multicolumn{10}{|c|}{ October } \\
\hline 05 & 1248 & 0.6 & 500 & 617 & 7.3 & 22.0 & $\cdots$ & 6.9 & 80 \\
\hline 05 & 1248 & 3.1 & 500 & 621 & 7.3 & 21.9 & $\cdots$ & 6.8 & 79 \\
\hline 05 & 1250 & 23 & 500 & 631 & 7.3 & 21.8 & $\cdots$ & 6.6 & 76 \\
\hline 05 & 1249 & 48 & 500 & 683 & 7.3 & 21.3 & $\cdots$ & 6.3 & 73 \\
\hline 19 & 1620 & 0.4 & 500 & 522 & 7.5 & 18.5 & $\cdots$ & 7.5 & 81 \\
\hline 19 & 1620 & 3.0 & 500 & 524 & 7.4 & 18.5 & $\cdots$ & 7.4 & 80 \\
\hline 19 & 1622 & 24 & 500 & 521 & 7.4 & 18.5 & - & 7.3 & 79 \\
\hline 19 & 1621 & 48 & 500 & 556 & 7.4 & 17.2 & $\cdots$ & 6.5 & 68 \\
\hline
\end{tabular}


Table 29. Water-quality data for station 390803081443501, Ohio River at river mile 202.8, June to October, 1995.

$\left[\mathrm{ft}=\mathrm{Eeet} ; \mu \mathrm{S} / \mathrm{cm}=\right.$ microsiemens per centimeter $;{ }^{\circ} \mathrm{C}=$ degrees Celsius;

$\mathrm{mg} / \mathrm{L}=\mathrm{milligrams}$ per liter; $\cdots$ = data not collected]

\begin{tabular}{|c|c|c|c|c|c|c|c|c|c|}
\hline Date & Time & $\begin{array}{l}\text { Sampling } \\
\text { depth } \\
\text { (ft) }\end{array}$ & $\begin{array}{c}\text { Sample } \\
\text { location } \\
\text { (ft trom } \\
\text { left bank) }\end{array}$ & $\begin{array}{l}\text { Specific } \\
\text { conduct. } \\
\text { ance } \\
(\mu \mathrm{S} / \mathrm{cm})\end{array}$ & $\begin{array}{l}\mathrm{pH} \\
\text { (stan- } \\
\text { dard } \\
\text { units) }\end{array}$ & $\begin{array}{c}\text { Temper- } \\
\text { ature, } \\
\text { water } \\
\left({ }^{\circ} \mathrm{C}\right)\end{array}$ & $\begin{array}{l}\text { Trans- } \\
\text { parency } \\
\text { (secchi } \\
\text { disk) } \\
\text { (ft) }\end{array}$ & $\begin{array}{l}\text { Dissolved } \\
\text { oxygen } \\
(\mathrm{mg} / \mathrm{L})\end{array}$ & $\begin{array}{c}\text { Dissolved } \\
\text { oxygen } \\
\text { (percent } \\
\text { satura- } \\
\text { tion) }\end{array}$ \\
\hline \multicolumn{10}{|l|}{ June } \\
\hline 29 & 1610 & 0.2 & 600 & 393 & 7.4 & 27.6 & $\cdots$ & 6.8 & 87 \\
\hline 29 & 1610 & 3.0 & 600 & 393 & 7.3 & 27.1 & $\cdots$ & 6.2 & 80 \\
\hline 29 & 1612 & 19 & 600 & 400 & 7.3 & 27.1 & - - & 6.2 & 80 \\
\hline 29 & 1611 & 37 & 600 & 400 & 7.3 & 27.1 & $\cdots$ & 6.3 & 80 \\
\hline \multicolumn{10}{|l|}{ July } \\
\hline 13 & 1856 & 0.4 & 600 & 468 & 8.9 & 30.3 & $\cdots$ & 13.4 & 181 \\
\hline 13 & 1856 & 3.5 & 600 & 467 & 8.9 & 29.6 & - & 13.9 & 186 \\
\hline 13 & 1857 & 16 & 600 & 480 & 7.7 & 27.2 & - & 7.3 & 94 \\
\hline 13 & 1858 & 36 & 600 & 482 & 7.6 & 27.0 & $-\cdot$ & 6.5 & 82 \\
\hline 27 & 1627 & 0.7 & 600 & 510 & 7.7 & 29.2 & $\cdots$ & 7.3 & 97 \\
\hline 27 & 1627 & 3.0 & 600 & 509 & 7.6 & 29.1 & $\cdots$ & 6.5 & 86 \\
\hline 27 & 1629 & 19 & 600 & 526 & 7.5 & 28.9 & -- & 6.0 & 79 \\
\hline 27 & 1628 & 38 & 600 & 524 & 7.4 & 28.7 & - & 5.6 & 74 \\
\hline \multicolumn{10}{|c|}{ August } \\
\hline 10 & 1650 & 0.9 & 600 & 457 & 7.6 & 27.8 & - & 6.5 & 84 \\
\hline 10 & 1651 & 3.5 & 600 & 460 & 7.5 & 27.2 & - & 6.2 & 79 \\
\hline 10 & 1652 & 18 & 600 & 455 & 7.5 & 26.7 & $\cdots$ & 5.8 & 74 \\
\hline 10 & 1651 & 37 & 600 & 456 & 7.4 & 26.6 & $\cdots$ & 5.7 & 73 \\
\hline 24 & 0955 & 0.7 & 600 & 496 & 7.7 & 29.6 & -- & 6.8 & 90 \\
\hline 24 & 0955 & 3.3 & 600 & 496 & 7.7 & 29.6 & $\cdots$ & 6.5 & 87 \\
\hline 24 & 0956 & 19 & 600 & 496 & 7.7 & 29.6 & $\cdots$ & 6.3 & 84 \\
\hline 24 & 0956 & 36 & 600 & 497 & 7.6 & 29.5 & $\cdots$ & 6.0 & 80 \\
\hline \multicolumn{10}{|c|}{ September } \\
\hline 06 & 1634 & 0.2 & 600 & 533 & 7.8 & 30.1 & $\cdots$ & 7.5 & 101 \\
\hline 06 & 1636 & 3.0 & 600 & 534 & 8.0 & 28.4 & -- & 7.7 & 100 \\
\hline 06 & 1637 & 16 & 600 & 536 & 7.4 & 27.6 & -- & 5.3 & 67 \\
\hline 06 & 1637 & 37 & 600 & 537 & 7.4 & 27.4 & - & 4.9 & 62 \\
\hline 20 & 1504 & 1.1 & 600 & 658 & 7.5 & 23.9 & $\cdots$ & 5.5 & 66 \\
\hline 20 & 1505 & 2.7 & 600 & 657 & 7.4 & 23.9 & - & 5.4 & 65 \\
\hline 20 & 1506 & 17 & 600 & 655 & 7.4 & 23.9 & $\cdots$ & 5.4 & 65 \\
\hline 20 & 1505 & 31 & 600 & 659 & 7.4 & 23.9 & & 5.4 & 65 \\
\hline
\end{tabular}


Table 29. Water-quality data for station 390803081443501, Ohio River at river mile 202.8, June to October, 1995, Continued.

[ft $=$ feet $; \mu \mathrm{s} / \mathrm{cm}=$ microsiemens per centimeter $;{ }^{\circ} \mathrm{C}=$ degrees Celsius;

$\mathrm{mg} / \mathrm{L}=\mathrm{milligrams}$ per liter;. = data not collected]

\begin{tabular}{|c|c|c|c|c|c|c|c|c|c|c|}
\hline Date & Time & $\begin{array}{l}\text { Sampling } \\
\text { depth } \\
\text { \{ft\} }\end{array}$ & $\begin{array}{r}\mathrm{Sa} \\
10 \mathrm{c} \\
1 \mathrm{tt} \\
\text { left }\end{array}$ & $\begin{array}{l}\text { ample } \\
\text { zation } \\
=\text { from } \\
\text { : bank) }\end{array}$ & $\begin{array}{l}\text { Specific } \\
\text { conduct- } \\
\text { ance } \\
(\mu \mathrm{S} / \mathrm{cm})\end{array}$ & $\begin{array}{l}\mathrm{pH} \\
\text { (stan- } \\
\text { dard } \\
\text { units) }\end{array}$ & $\begin{array}{c}\text { Temper- } \\
\text { ature, } \\
\text { water } \\
\left({ }^{\circ} \mathrm{C}\right)\end{array}$ & $\begin{array}{l}\text { Trans- } \\
\text { parency } \\
\text { (Secchl } \\
\text { disk) } \\
(\mathrm{ft})\end{array}$ & $\begin{array}{l}\text { Dissolved } \\
\text { oxygen } \\
\text { (mg/L) }\end{array}$ & $\begin{array}{c}\text { Dissolved } \\
\text { oxygen } \\
\text { (percent } \\
\text { satura- } \\
\text { tion) }\end{array}$ \\
\hline \multicolumn{11}{|c|}{ October } \\
\hline 05 & 1256 & 0.5 & & 600 & 636 & 7.3 & 22.0 & $\cdots$ & 6.8 & 80 \\
\hline 05 & 1256 & 2.9 & & 600 & 640 & 7.3 & 22.0 & - & 6.8 & 79 \\
\hline 05 & 1258 & 17 & & 600 & 649 & 7.3 & 21.9 & - & 6.7 & 78 \\
\hline 05 & 1257 & 34 & & 600 & 652 & 7.3 & 21.9 & $\cdots$ & 6.6 & 76 \\
\hline 19 & 1630 & 0.5 & & 600 & 512 & 7.5 & 18.8 & $\cdots$ & 7.4 & 81 \\
\hline 19 & 1630 & 3.3 & & 600 & 510 & 7.4 & 18.7 & $\cdots$ & 7.4 & 80 \\
\hline 19 & 1632 & 19 & & 600 & 512 & 7.4 & 18.3 & $-\cdot$ & 7.1 & 76 \\
\hline 19 & 1631 & 35 & & 600 & 512 & 7.4 & 18.3 & $\cdots$ & 7.0 & 75 \\
\hline
\end{tabular}


Table 30. Water-quality data for station 390721081443001, Ohio River at river mile 203.6, June to October, 1995.

$\mathrm{ft}=$ feet; $\mu \mathrm{s} / \mathrm{cm}=$ microsiemens per centimeter; ${ }^{\circ} \mathrm{C}=$ degrees Celsius;

$\mathrm{mg} / \mathrm{L}=$ milligrams per liter; - - = data not collected]

\begin{tabular}{|c|c|c|c|c|c|c|c|c|c|}
\hline Date & Time & $\begin{array}{l}\text { Sampling } \\
\text { depth } \\
\text { (ft) }\end{array}$ & $\begin{array}{c}\text { Sample } \\
\text { location } \\
\text { ( } \mathrm{ft} \text { trom } \\
\text { left bank) }\end{array}$ & $\begin{array}{l}\text { Specific } \\
\text { conduct- } \\
\text { ance } \\
(\mu \mathrm{S} / \mathrm{cm})\end{array}$ & $\begin{array}{l}\mathrm{pH} \\
\text { (stan- } \\
\text { dard } \\
\text { units) }\end{array}$ & $\begin{array}{c}\text { Temper- } \\
\text { ature, } \\
\text { water } \\
\left({ }^{\circ} \mathrm{C}\right)\end{array}$ & $\begin{array}{c}\text { Trans- } \\
\text { parency } \\
\text { (Secchi } \\
\text { disk) } \\
\text { (ft) }\end{array}$ & $\begin{array}{l}\text { Dissolved } \\
\text { oxygen } \\
(\mathrm{mg} / \mathrm{L})\end{array}$ & $\begin{array}{c}\text { Dissolved } \\
\text { oxygen } \\
\text { (percent } \\
\text { satura- } \\
\text { tion) }\end{array}$ \\
\hline
\end{tabular}

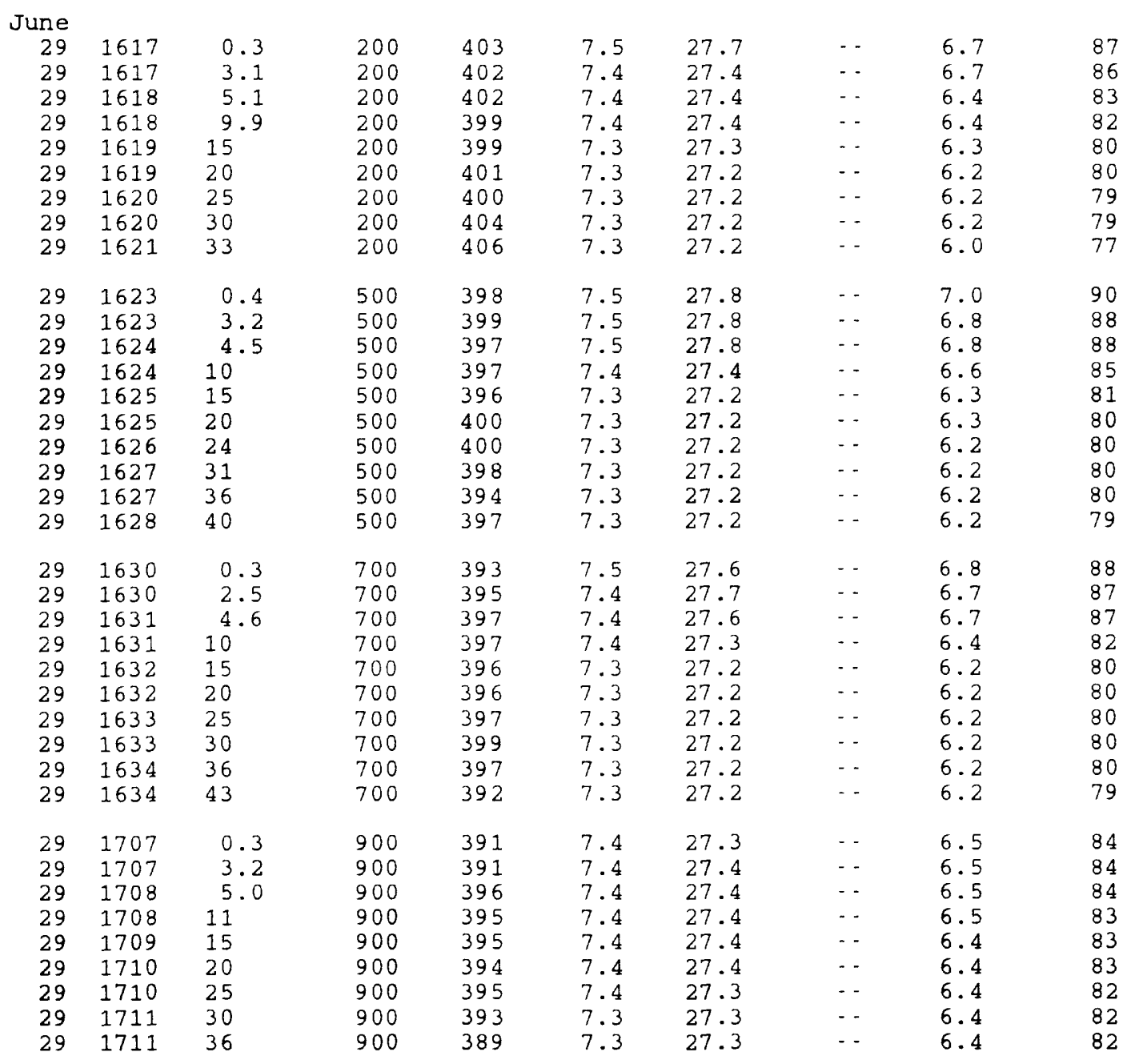


Table 30. Water-quality data for station 390721081443001, Ohio River at river mile 203.6, June to october, 1995, Continued.

[ft $=$ feet $; \mu \mathrm{s} / \mathrm{cm}=$ microsiemens per centimeter $;{ }^{\circ} \mathrm{C}=$ degrees Celsius;

$\mathrm{mg} / \mathrm{L}=\mathrm{milligrams}$ per liter;.. = data not collected]

\begin{tabular}{|c|c|c|c|c|c|c|c|c|c|}
\hline Date & Time & $\begin{array}{l}\text { Sampl ing } \\
\text { depth } \\
\text { (Et) }\end{array}$ & $\begin{array}{c}\text { Sample } \\
\text { location } \\
\text { (ft from } \\
\text { left bank) }\end{array}$ & $\begin{array}{l}\text { Specific } \\
\text { conduct- } \\
\text { ance } \\
(\mu \mathrm{S} / \mathrm{cm})\end{array}$ & $\begin{array}{l}\mathrm{pH} \\
\text { (stan- } \\
\text { dard } \\
\text { units) }\end{array}$ & $\begin{array}{l}\text { Temper- } \\
\text { ature, } \\
\text { water } \\
\left({ }^{\circ} \mathrm{C}\right)\end{array}$ & $\begin{array}{c}\text { Trans- } \\
\text { parency } \\
\text { (Secchl } \\
\text { disk) } \\
\text { (Et) }\end{array}$ & $\begin{array}{l}\text { Dissolved } \\
\text { oxygen } \\
(\mathrm{mg} / \mathrm{L})\end{array}$ & $\begin{array}{c}\text { Dissolved } \\
\text { oxygen } \\
\text { (percent } \\
\text { satura- } \\
\text { tion) }\end{array}$ \\
\hline
\end{tabular}

$\begin{array}{rlllllllrr}\text { July } & & & & & & \\ 13 & 1904 & 0.7 & 200 & 460 & 9.0 & 30.7 & \ldots & 13.6 & 184 \\ 13 & 1905 & 2.8 & 200 & 464 & 8.7 & 28.9 & \ldots & 11.9 & 157 \\ 13 & 1905 & 4.9 & 200 & 463 & 8.4 & 28.2 & \ldots & 10.7 & 139 \\ 13 & 1906 & 9.9 & 200 & 465 & 8.0 & 27.7 & \ldots & 9.1 & 118 \\ 13 & 1907 & 15 & 200 & 466 & 7.7 & 27.4 & \ldots & 7.4 & 95 \\ 13 & 1908 & 20 & 200 & 462 & 7.6 & 27.2 & \ldots & 6.7 & 86 \\ 13 & 1908 & 25 & 200 & 468 & 7.6 & 27.1 & \ldots & 6.3 & 80 \\ 13 & 1909 & 30 & 200 & 472 & 7.5 & 27.1 & \ldots & 6.1 & 78 \\ 13 & 1910 & 32 & 200 & 471 & 7.5 & 27.1 & \ldots & 6.0 & 76 \\ 13 & 1913 & 0.6 & 500 & 460 & 9.0 & 30.3 & \ldots & 14.2 & 191 \\ 13 & 1914 & 3.0 & 500 & 464 & 8.8 & 29.2 & \ldots & 12.0 & 159 \\ 13 & 1914 & 5.0 & 500 & 474 & 8.3 & 28.0 & \ldots & 10.2 & 132 \\ 13 & 1915 & 9.9 & 500 & 476 & 8.0 & 27.6 & \ldots & 8.9 & 115 \\ 13 & 1916 & 15 & 500 & 479 & 7.8 & 27.4 & \ldots & 8.0 & 102 \\ 13 & 1916 & 20 & 500 & 478 & 7.7 & 27.2 & \ldots & 7.0 & 90 \\ 13 & 1917 & 25 & 500 & 475 & 7.6 & 27.1 & \ldots & 6.7 & 86 \\ 13 & 1918 & 30 & 500 & 472 & 7.6 & 27.1 & \ldots & 6.5 & 83 \\ 13 & 1918 & 35 & 500 & 469 & 7.6 & 27.1 & \ldots & 6.5 & 83 \\ 13 & 1919 & 40 & 500 & 480 & 7.6 & 27.1 & \ldots & 6.4 & 82 \\ 13 & 1925 & 0.7 & 700 & 465 & 8.9 & 30.0 & \ldots & 13.4 & 181 \\ 13 & 1925 & 3.1 & 700 & 468 & 8.7 & 28.9 & \ldots & 12.3 & 163 \\ 13 & 1926 & 5.0 & 700 & 474 & 8.3 & 28.3 & \ldots & 10.1 & 132 \\ 13 & 1927 & 10 & 700 & 471 & 7.9 & 27.6 & \ldots & 8.0 & 103 \\ 13 & 1928 & 15 & 700 & 473 & 7.7 & 27.3 & \ldots & 7.3 & 93 \\ 13 & 1928 & 20 & 700 & 476 & 7.7 & 27.2 & \ldots & 7.2 & 92 \\ 13 & 1929 & 24 & 700 & 472 & 7.7 & 27.2 & \ldots & 7.2 & 92 \\ 13 & 1930 & 30 & 700 & 476 & 7.7 & 27.1 & \ldots & 6.9 & 88 \\ 13 & 1930 & 35 & 700 & 484 & 7.6 & 27.1 & \ldots & 6.8 & 87 \\ 13 & 1931 & 40 & 700 & 482 & 7.6 & 27.1 & \ldots & 6.6 & 84 \\ 13 & 1932 & 45 & 700 & 474 & 7.6 & 27.1 & \ldots & 6.6 & 84\end{array}$


Table 30. Water-quality data for station 390721081443001, Ohio River at river mile 203.6, June to October, 1995, Continued.

$\left[\mathrm{Et}=\mathrm{feet} ; \mu \mathrm{s} / \mathrm{cm}=\right.$ microsiemens per centimeter; ${ }^{\circ} \mathrm{C}=$ degrees Celsius:

$\mathrm{mg} / \mathrm{L}=\mathrm{milligrams}$ per liter; $\cdot-$ = data not collected]

\begin{tabular}{|c|c|c|c|c|c|c|c|c|c|}
\hline Date & Time & $\begin{array}{l}\text { Sampling } \\
\text { depth } \\
\text { (ft) }\end{array}$ & $\begin{array}{l}\text { Sample } \\
\text { location } \\
\text { (ft from } \\
\text { left bank) }\end{array}$ & $\begin{array}{l}\text { Specific } \\
\text { conduct- } \\
\text { ance } \\
(\mu \mathrm{s} / \mathrm{cm})\end{array}$ & $\begin{array}{l}\mathrm{pH} \\
\text { (stan- } \\
\text { dard } \\
\text { units) }\end{array}$ & $\begin{array}{c}\text { Temper- } \\
\text { ature, } \\
\text { water } \\
\left({ }^{\circ} \mathrm{C}\right)\end{array}$ & $\begin{array}{l}\text { Trans- } \\
\text { parency } \\
\text { (secchi } \\
\text { disk) } \\
( \pm t)\end{array}$ & $\begin{array}{c}\text { Dissolved } \\
\text { oxygen } \\
\text { (mg/L) }\end{array}$ & $\begin{array}{c}\text { Dissolved } \\
\text { oxygen } \\
\text { (percent } \\
\text { satura- } \\
\text { tron) }\end{array}$ \\
\hline
\end{tabular}

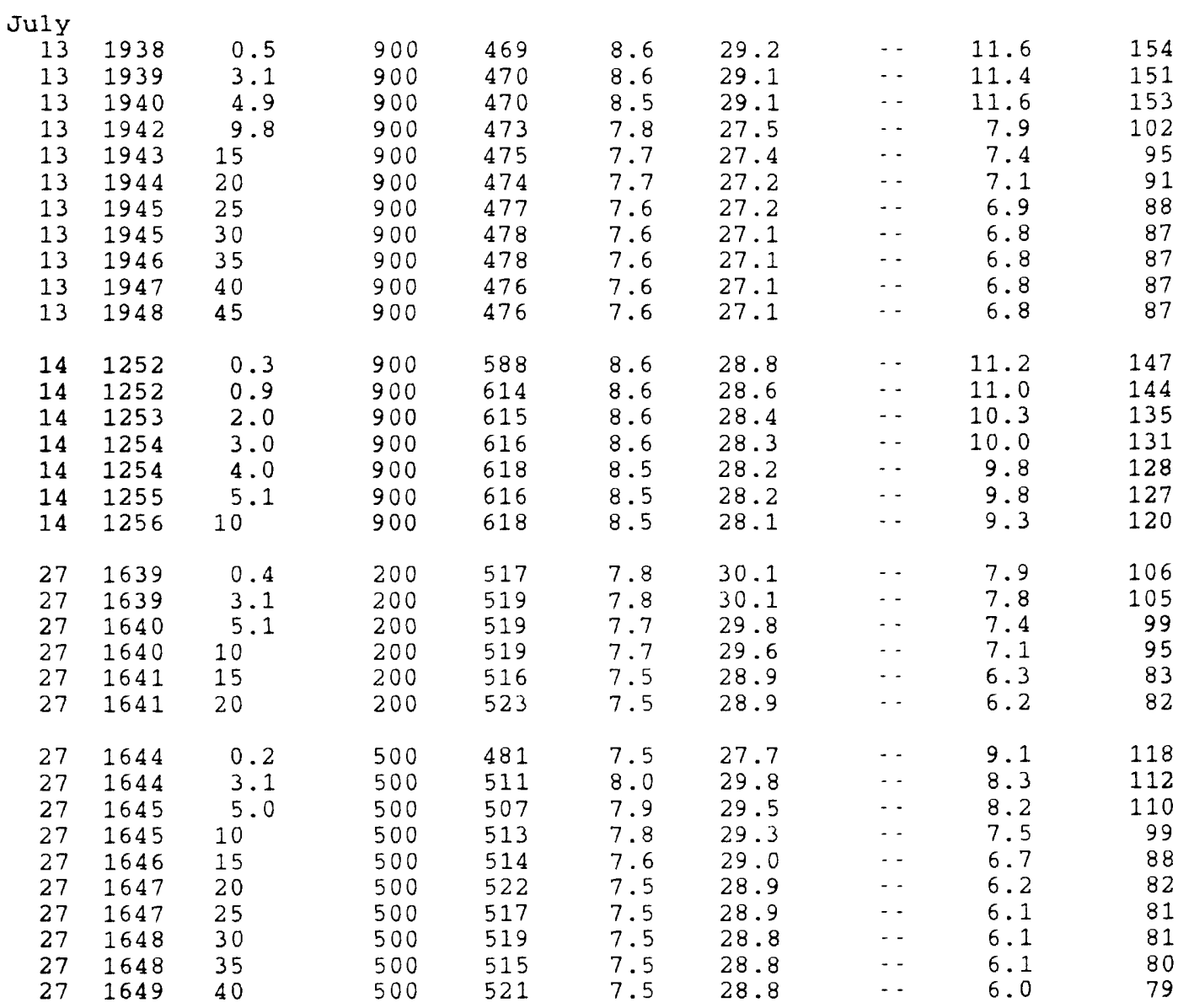


Table 30. Water-quality data for station 390721081443001, Ohio River at river mile 203.6, June to October, 1995, Continued.

[ft $=$ feet $; \mu \mathrm{s} / \mathrm{cm}=$ microsiemens per centımeter; ${ }^{\circ} \mathrm{C}=$ degrees Celsius;

$\mathrm{mg} / \mathrm{L}=\mathrm{milligrams}$ per liter: - = data not collected]

\begin{tabular}{|c|c|c|c|c|c|c|c|c|c|}
\hline Date & Time & $\begin{array}{l}\text { Samp 1 ing } \\
\text { depth } \\
\text { (Et) }\end{array}$ & $\begin{array}{c}\text { Sample } \\
\text { location } \\
\text { (ft from } \\
\text { left bank) }\end{array}$ & $\begin{array}{l}\text { Specific } \\
\text { conduct- } \\
\text { ance } \\
(\mu \mathrm{s} / \mathrm{cm})\end{array}$ & $\begin{array}{l}\mathrm{pH} \\
\text { (stan- } \\
\text { dard } \\
\text { units) }\end{array}$ & $\begin{array}{c}\text { Temper- } \\
\text { ature, } \\
\text { water } \\
\left({ }^{\circ} \mathrm{C}\right)\end{array}$ & $\begin{array}{l}\text { Trans - } \\
\text { parency } \\
\text { (secchi } \\
\text { disk) } \\
(t t)\end{array}$ & $\begin{array}{c}\text { Dussolved } \\
\text { oxygen } \\
(\mathrm{mg} / \mathrm{L})\end{array}$ & $\begin{array}{c}\text { Dissolved } \\
\text { oxygen } \\
\text { (percent } \\
\text { satura- } \\
\text { tion) }\end{array}$ \\
\hline
\end{tabular}

\begin{tabular}{|c|c|c|c|c|c|c|c|c|c|}
\hline \multicolumn{10}{|l|}{ July } \\
\hline 27 & 1653 & 0.3 & 700 & 516 & 7.7 & 29.1 & $\cdots$ & 7.0 & 93 \\
\hline 27 & 1653 & 3.1 & 700 & 521 & 7.6 & 29.2 & $\cdots$ & 6.9 & 92 \\
\hline 27 & 1654 & 5.1 & 700 & 515 & 7.6 & 29.1 & - - & 6.6 & 88 \\
\hline 27 & 1654 & 10 & 700 & 509 & 7.6 & 29.0 & $\cdots$ & 6.5 & 86 \\
\hline 27 & 1655 & 15 & 700 & 517 & 7.5 & 29.0 & - & 6.4 & 34 \\
\hline 27 & 1655 & 20 & 700 & 520 & 7.5 & 29.0 & $\cdots$ & 6.3 & 84 \\
\hline 27 & 1656 & 25 & 700 & 520 & 7.5 & 28.9 & $\cdots$ & 6.2 & 4 \\
\hline 27 & 1656 & 30 & 700 & 511 & 7.5 & 28.9 & $\cdots$ & 6.1 & \\
\hline 27 & 1657 & 35 & 700 & 512 & 7.5 & 28.9 & - & 6.1 & \\
\hline 27 & 1657 & 40 & 700 & 525 & 7.5 & 28.8 & $\cdots$ & 6.1 & $\varepsilon$ \\
\hline 27 & 1658 & 45 & 700 & 515 & 7.5 & 28.8 & - & 6.0 & \\
\hline 27 & 1701 & 0.3 & 900 & 520 & 7.6 & 29.1 & $\cdots$ & 6.6 & 1 \\
\hline 27 & 1701 & 3.1 & 900 & 519 & 7.6 & 29.1 & $\cdots$ & 6.6 & \\
\hline 27 & 1702 & 5.0 & 900 & 520 & 7.5 & 29.0 & - - & 6.4 & \\
\hline 27 & 1702 & 10 & 900 & 522 & 7.5 & 29.0 & $\cdots$ & 6.4 & \\
\hline 27 & 1703 & 15 & 900 & 518 & 7.5 & 29.0 & $\cdots$ & 6.4 & \\
\hline 27 & 1703 & 20 & 900 & 513 & 7.5 & 29.0 & $\cdots$ & 6.4 & \\
\hline 27 & 1704 & 25 & 900 & 527 & 7.5 & 29.0 & - - & 6.4 & \\
\hline 27 & 1704 & 31 & 900 & 525 & 7.5 & 29.0 & - - & 6.3 & \\
\hline 27 & 1705 & 36 & 900 & 526 & 7.5 & 28.9 & $\cdots$ & 6.3 & \\
\hline 27 & 1705 & 41 & 900 & 513 & 7.5 & 28.9 & $\cdots$ & 6.1 & \\
\hline 27 & 1706 & 45 & 900 & 519 & 7.5 & 28.9 & $\cdots$ & 6.1 & \\
\hline Augus & & & & & & & & & \\
\hline 10 & 1657 & 1.2 & 200 & 439 & 7.6 & 28.3 & $\cdots$ & 6.5 & \\
\hline 10 & 1657 & 3.5 & 200 & 439 & 7.5 & 27.1 & $\cdots$ & 5.9 & \\
\hline 10 & 1658 & 5.5 & 200 & 440 & 7.4 & 27.0 & $\cdots$ & 5.9 & \\
\hline 10 & 1658 & 10 & 200 & 443 & 7.4 & 26.7 & $\cdots$ & 5.8 & \\
\hline 10 & 1659 & 15 & 200 & 443 & 7.4 & 26.6 & $\cdots$ & 5.8 & \\
\hline 10 & 1659 & 20 & 200 & 443 & 7.4 & 26.6 & $\cdots$ & 5.7 & \\
\hline 10 & 1700 & 25 & 200 & 443 & 7.4 & 26.6 & $\cdots$ & 5.7 & \\
\hline 10 & 1700 & 30 & 200 & 442 & 7.4 & 26.6 & $\cdots$ & 5.7 & \\
\hline 10 & 1701 & 34 & 200 & 442 & 7.4 & 26.6 & - & 5.6 & \\
\hline
\end{tabular}


Table 30. Water-quality data for station 390721081443001, Ohio River at river mile 203.6, June to October, 1995, Continued.

$\left[\mathrm{ft}=\mathrm{feet} ; \mu \mathrm{S} / \mathrm{cm}=\mathrm{m}\right.$ crosiemens per centimeter; ${ }^{\circ} \mathrm{C}=$ degrees celsius;

$\mathrm{mg} / \mathrm{L}=$ militigrams per liter: - = data not collected]

\begin{tabular}{|c|c|c|c|c|c|c|c|c|c|}
\hline Date & Time & $\begin{array}{l}\text { Sampling } \\
\text { depth } \\
\text { (ft) }\end{array}$ & $\begin{array}{l}\text { Sample } \\
\text { location } \\
\text { (ft from } \\
\text { left bank) }\end{array}$ & $\begin{array}{l}\text { Specific } \\
\text { conduct- } \\
\text { ance } \\
(\mu \mathrm{S} / \mathrm{cm})\end{array}$ & $\begin{array}{l}\mathrm{pH} \\
\text { (stan- } \\
\text { dard } \\
\text { units) }\end{array}$ & $\begin{array}{c}\text { Temper } \\
\text { ature, } \\
\text { water } \\
\left({ }^{\circ} \mathrm{C}\right)\end{array}$ & $\begin{array}{l}\text { Trans- } \\
\text { parency } \\
\text { (Secchl } \\
\text { disk) } \\
\text { (tt) }\end{array}$ & $\begin{array}{c}\text { Dissolved } \\
\text { oxygen } \\
(\mathrm{mg} / \mathrm{L})\end{array}$ & $\begin{array}{c}\text { Dissolved } \\
\text { oxygen } \\
\text { (percent } \\
\text { satura- } \\
\text { tion) }\end{array}$ \\
\hline
\end{tabular}

\begin{tabular}{|c|c|c|c|c|c|c|c|c|c|}
\hline \multicolumn{10}{|c|}{ August } \\
\hline 10 & 1705 & 0.9 & 500 & 446 & 7.6 & 28.1 & $\cdots$ & 6.6 & 86 \\
\hline 10 & 1705 & 3.5 & 500 & 444 & 7.5 & 27.5 & $\cdots$ & 6.3 & 81 \\
\hline 10 & 1706 & 5.5 & 500 & 447 & 7.5 & 27.0 & $\cdots$ & 6.0 & 77 \\
\hline 10 & 1706 & 10 & 500 & 447 & 7.4 & 26.7 & $\ldots$ & 5.9 & 76 \\
\hline 10 & 1707 & 15 & 500 & 447 & 7.4 & 26.6 & $\cdots$ & 5.8 & 74 \\
\hline 10 & 1707 & 20 & 500 & 444 & 7.4 & 26.6 & $\cdots$ & 5.8 & 74 \\
\hline 10 & 1708 & 25 & 500 & 443 & 7.4 & 26.6 & $\cdots$ & 5.8 & 73 \\
\hline 10 & 1708 & 30 & 500 & 436 & 7.4 & 26.6 & $\cdots$ & 5.8 & 73 \\
\hline 10 & 1709 & 35 & 500 & 443 & 7.4 & 26.5 & - & 5.7 & 73 \\
\hline 10 & 1709 & 39 & 500 & 437 & 7.4 & 26.5 & $\cdots$ & 5.7 & 73 \\
\hline 10 & 1712 & 0.8 & 700 & 450 & 7.6 & 27.6 & $\cdots$ & 6.6 & 86 \\
\hline 10 & 1712 & 3.4 & 700 & 449 & 7.5 & 27.1 & $\cdots$ & 6.3 & 80 \\
\hline 10 & 1713 & 5.4 & 700 & 448 & 7.5 & 27.0 & $\cdots$ & 6.1 & 8 \\
\hline 10 & 1713 & 10 & 700 & 451 & 7.5 & 26.8 & $\cdots$ & 6.0 & 76 \\
\hline 10 & 1714 & 15 & 700 & 455 & 7.4 & 26.6 & $\cdots$ & 5.9 & 75 \\
\hline 10 & 1714 & 20 & 700 & 445 & 7.4 & 26.6 & $\cdots$ & 5.8 & 74 \\
\hline 10 & 1715 & 24 & 700 & 454 & 7.4 & 26.6 & $\cdots$ & 5.8 & 4 \\
\hline 10 & 1715 & 29 & 700 & 438 & 7.4 & 26.6 & $\cdots$ & 5.8 & 74 \\
\hline 10 & 1716 & 34 & 700 & 449 & 7.4 & 26.6 & $\cdots$ & 5.8 & 74 \\
\hline 10 & 1716 & 39 & 700 & 448 & 7.4 & 26.6 & $\cdots$ & 5.8 & 73 \\
\hline 10 & 1717 & 44 & 700 & 447 & 7.4 & 26.6 & $\cdots$ & 5.7 & 73 \\
\hline 10 & 1720 & 0.9 & 900 & 451 & 7.5 & 26.9 & $\cdots$ & 6.1 & 78 \\
\hline 10 & 1721 & 3.4 & 900 & 451 & 7.5 & 26.8 & $\cdots$ & 6.0 & 6 \\
\hline 10 & 1721 & 5.5 & 900 & 448 & 7.5 & 26.8 & $\cdots$ & 5.9 & 6 \\
\hline 10 & 1722 & 10 & 900 & 446 & 7.4 & 26.7 & $\cdots$ & 5.9 & 75 \\
\hline 10 & 1722 & 15 & 900 & 443 & 7.4 & 26.7 & $\cdots$ & 5.9 & 75 \\
\hline 10 & 1723 & 20 & 900 & 445 & 7.4 & 26.7 & $\cdots$ & 5.8 & 74 \\
\hline 10 & 1723 & 25 & 900 & 447 & 7.4 & 26.7 & $\cdots$ & 5.8 & 74 \\
\hline 10 & 1724 & 30 & 900 & 443 & 7.4 & 26.7 & $\cdots$ & 5.8 & 7 \\
\hline 10 & 1724 & 35 & 900 & 449 & 7.4 & 26.7 & $\cdots$ & 5.8 & 74 \\
\hline 10 & 1725 & 40 & 900 & 447 & 7.4 & 26.6 & $\cdots$ & 5.8 & 74 \\
\hline 10 & 1725 & 44 & 900 & 442 & 7.4 & 26.6 & $\cdots$ & 5.8 & 73 \\
\hline 10 & 1726 & 47 & 900 & 446 & 7.4 & 26.6 & $\cdots$ & 5.7 & 73 \\
\hline
\end{tabular}


Table 30. Water-quality data for station 390721081443001, Ohio River at river mile 203.6, June to October, 1995, Continued.

[ft $=$ feet $\mu \mathrm{S} / \mathrm{cm}=$ microsiemens per centimeter: ${ }^{\circ} \mathrm{C}=$ degrees Celsius;

$\mathrm{mg} / \mathrm{L}=$ milligrams per liter; . - = data not collected]

\begin{tabular}{|c|c|c|c|c|c|c|c|c|c|}
\hline Date & Time & $\begin{array}{l}\text { Sampling } \\
\text { depth } \\
\text { (Et) }\end{array}$ & $\begin{array}{c}\text { Sample } \\
\text { location } \\
\text { (Et from } \\
\text { left bank) }\end{array}$ & $\begin{array}{l}\text { Specific } \\
\text { conduct- } \\
\text { ance } \\
(\mu \mathrm{s} / \mathrm{cm})\end{array}$ & $\begin{array}{l}\text { pH } \\
\text { (stan- } \\
\text { dard } \\
\text { units) }\end{array}$ & $\begin{array}{c}\text { Temper- } \\
\text { ature. } \\
\text { water } \\
\left({ }^{\circ} \mathrm{C}\right)\end{array}$ & $\begin{array}{c}\text { Trans } \\
\text { parency } \\
\text { (secchI } \\
\text { disk) } \\
(\mathrm{Et})\end{array}$ & $\begin{array}{l}\text { Dissolved } \\
\text { oxygen } \\
\text { (mg/L) }\end{array}$ & $\begin{array}{c}\text { Dissolved } \\
\text { oxygen } \\
\text { (percent } \\
\text { satura- } \\
\text { tion) }\end{array}$ \\
\hline
\end{tabular}

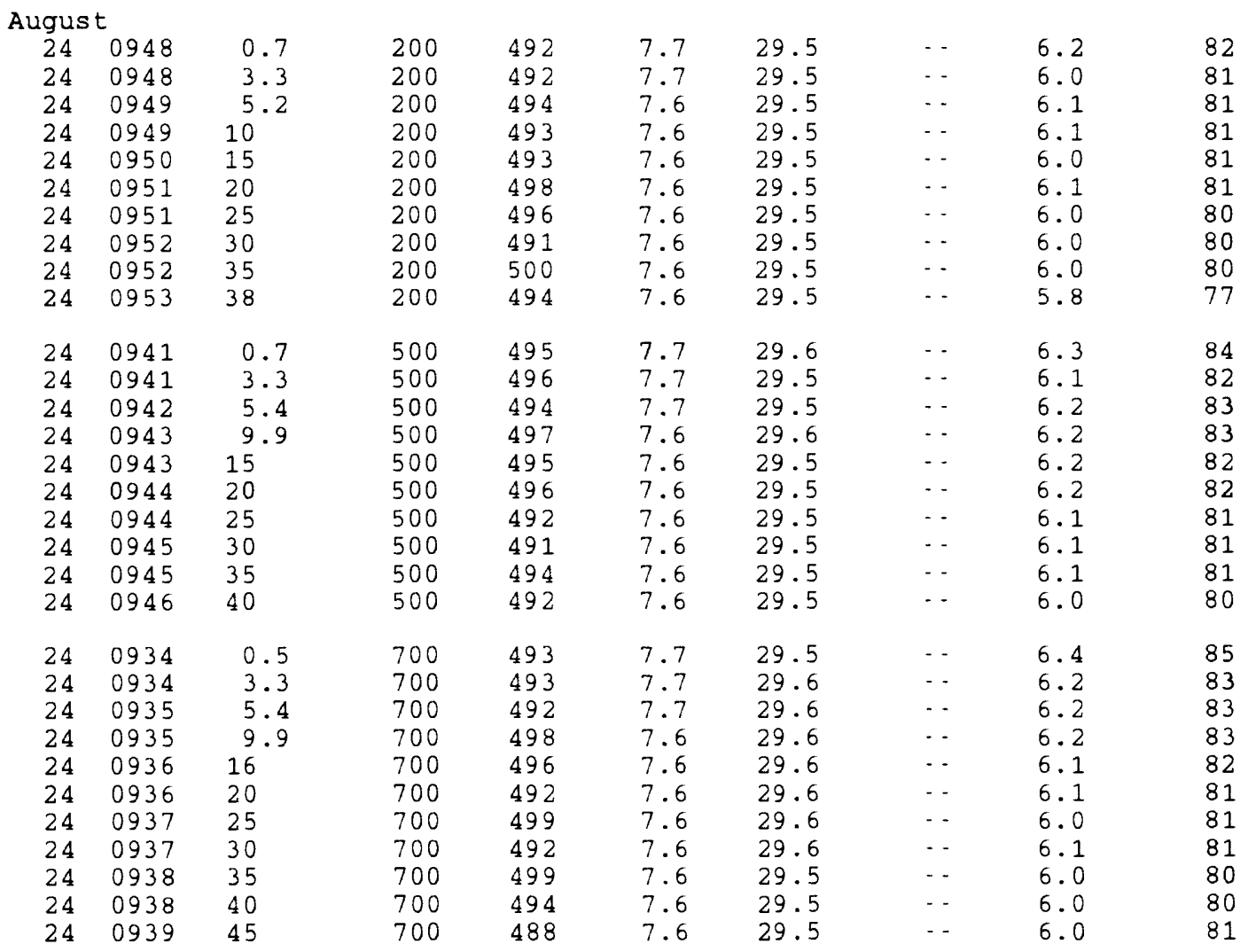


Table 30. Water-quality data for station 390721081443001, Ohio River at river mile 203.6, June to October, 1995, Continued.

[ft $=$ feet; $\mu \mathrm{S} / \mathrm{cm}=$ microsiemens per centimeter; ${ }^{\circ} \mathrm{C}=$ degrees celsius;

$\mathrm{mg} / \mathrm{L}=$ mililigrams per liter: - = data not collected]

\begin{tabular}{|c|c|c|c|c|c|c|c|c|c|}
\hline Date & Time & $\begin{array}{l}\text { Sampling } \\
\text { depth } \\
\text { (ft) }\end{array}$ & $\begin{array}{l}\text { Sample } \\
\text { location } \\
\text { (ft trom } \\
\text { left bank) }\end{array}$ & $\begin{array}{l}\text { Specific } \\
\text { conduct- } \\
\text { ance } \\
(\mu S / \mathrm{cm})\end{array}$ & $\begin{array}{l}\text { pH } \\
\text { (stan- } \\
\text { dard } \\
\text { units) }\end{array}$ & $\begin{array}{c}\text { Temper- } \\
\text { ature, } \\
\text { water } \\
\left({ }^{\circ} \mathrm{C}\right)\end{array}$ & $\begin{array}{l}\text { Trans- } \\
\text { parency } \\
\text { (Secchi } \\
\text { disk) } \\
\text { (ft) }\end{array}$ & $\begin{array}{c}\text { Dissolved } \\
\text { oxygen } \\
(\mathrm{mg} / \mathrm{L})\end{array}$ & $\begin{array}{c}\text { Dissolved } \\
\text { oxygen } \\
\text { (percent } \\
\text { satura- } \\
\text { tion) }\end{array}$ \\
\hline
\end{tabular}

\begin{tabular}{|c|c|c|c|c|c|c|c|c|c|}
\hline \multicolumn{10}{|c|}{ August } \\
\hline 24 & 0926 & 0.5 & 900 & 494 & 7.7 & 29.5 & $\cdots$ & 6.5 & 86 \\
\hline 24 & 0926 & 3.1 & 900 & 495 & 7.7 & 29.5 & $\cdots$ & 6.2 & 82 \\
\hline 24 & 0927 & 5.1 & 900 & 494 & 7.6 & 29.6 & $\cdots$ & 6.1 & 82 \\
\hline 24 & 0927 & 9.8 & 900 & 495 & 7.6 & 29.6 & $\cdots$ & 6.0 & 80 \\
\hline 24 & 0928 & 15 & 900 & 496 & 7.6 & 29.6 & $\cdots$ & 6.0 & 80 \\
\hline 24 & 0928 & 20 & 900 & 493 & 7.6 & 29.6 & $\cdots$ & 6.1 & 81 \\
\hline 24 & 0929 & 25 & 900 & 493 & 7.6 & 29.6 & $\cdots$ & 6.1 & 81 \\
\hline 24 & 0929 & 30 & 900 & 497 & 7.6 & 29.6 & $\cdots$ & 6.1 & 81 \\
\hline 24 & 0930 & 35 & 900 & 492 & 7.6 & 29.6 & - & 6.1 & 81 \\
\hline 24 & 0930 & 40 & 900 & 492 & 7.6 & 29.6 & $\cdots$ & 6.1 & 81 \\
\hline 24 & 0931 & 45 & 900 & 492 & 7.6 & 29.6 & $\cdots$ & 6.1 & 81 \\
\hline 24 & 0931 & 49 & 900 & 499 & 7.7 & 29.6 & $\cdots$ & 6.0 & 81 \\
\hline Sept & ber & & & & & & & & \\
\hline 06 & 1705 & 0.2 & 200 & 530 & 7.9 & 30.4 & $\cdots$ & 8.3 & 111 \\
\hline 06 & 1705 & 3.0 & 200 & 530 & 7.8 & 28.7 & - & 7.5 & 97 \\
\hline 06 & 1706 & 6.6 & 200 & 531 & 7.4 & 27.8 & $\cdots$ & 5.5 & 71 \\
\hline 06 & 1706 & 9.7 & 200 & 529 & 7.4 & 27.7 & $\cdots$ & 5.2 & 67 \\
\hline 06 & 1707 & 15 & 200 & 527 & 7.4 & 27.6 & $\cdots$ & 5.0 & 65 \\
\hline 06 & 1707 & 20 & 200 & 529 & 7.4 & 27.6 & -- & 5.0 & 64 \\
\hline 06 & 1708 & 25 & 200 & 534 & 7.4 & 27.5 & $\cdots$ & 5.0 & 64 \\
\hline 06 & 1708 & 30 & 200 & 529 & 7.4 & 27.5 & $\cdots$ & 4.9 & 63 \\
\hline 06 & 1709 & 34 & 200 & 534 & 7.3 & 27.5 & $\cdots$ & 4.8 & 61 \\
\hline 06 & 1659 & 0.2 & 500 & 534 & 7.8 & 30.1 & $\cdots$ & 7.9 & 106 \\
\hline 06 & 1659 & 2.9 & 500 & 531 & 8.1 & 28.5 & $\cdots$ & 8.8 & 114 \\
\hline 06 & 1700 & 4.8 & 500 & 532 & 7.7 & 28.1 & - & 7.1 & 92 \\
\hline 06 & 1700 & 9.2 & 500 & 530 & 7.5 & 27.8 & $\cdots$ & 5.9 & 76 \\
\hline 06 & 1701 & 15 & 500 & 533 & 7.4 & 27.6 & $\cdots$ & 5.4 & 69 \\
\hline 06 & 1701 & 20 & 500 & 533 & 7.4 & 27.6 & $\cdots$ & 5.3 & 67 \\
\hline 06 & 1702 & 24 & 500 & 531 & 7.4 & 27.5 & $\cdots$ & 5.2 & 66 \\
\hline 06 & 1702 & 30 & 500 & 535 & 7.4 & 27.5 & $\cdots$ & 5.1 & 6 \\
\hline 06 & 1703 & 35 & 500 & 534 & 7.4 & 27.5 & $\cdots$ & 5.1 & 6 \\
\hline 06 & 1703 & 40 & 500 & 529 & 7.4 & 27.5 & $\cdots$ & 5.0 & 64 \\
\hline
\end{tabular}


Table 30. Water-quality data for station 390721081443001, Ohio River at river mile 203.6, June to October, 1995, Continued.

lft = feet; $\mu \mathrm{S} / \mathrm{cm}=$ microsiemens per centimeter; ${ }^{\circ} \mathrm{C}=$ degrees Celsius;

$\mathrm{mg} / L=\mathrm{milligrams}$ per liter: $\cdot$ = data not collected]

\begin{tabular}{|c|c|c|c|c|c|c|c|c|c|}
\hline Date & Time & $\begin{array}{l}\text { Sampling } \\
\text { depth } \\
\text { (ft) }\end{array}$ & $\begin{array}{c}\text { Sample } \\
\text { location } \\
\text { (ft from } \\
\text { left bank) }\end{array}$ & $\begin{array}{l}\text { Specific } \\
\text { conduct- } \\
\text { ance } \\
(\mu \mathrm{S} / \mathrm{cm})\end{array}$ & $\begin{array}{l}\text { pH } \\
\text { (stan- } \\
\text { dard } \\
\text { units) }\end{array}$ & $\begin{array}{c}\text { Temper- } \\
\text { ature, } \\
\text { water } \\
\left({ }^{\circ} \mathrm{C}\right)\end{array}$ & $\begin{array}{l}\text { Trans - } \\
\text { parency } \\
\text { (secchl } \\
\text { disk) } \\
(E t)\end{array}$ & $\begin{array}{l}\text { Dissolved } \\
\text { oxygen } \\
(\mathrm{mg} / \mathrm{L})\end{array}$ & $\begin{array}{c}\text { Dissolved } \\
\text { oxygen } \\
\text { (percent } \\
\text { satura- } \\
\text { tion) }\end{array}$ \\
\hline
\end{tabular}

\section{Sep tember}

\section{$06 \quad 1651$}

$06 \quad 1651$

$06 \quad 1656$

$06 \quad 1652$

$06 \quad 1652$

061653

$06 \quad 1653$

$06 \quad 1654$

$06 \quad 1654$

061655

061655

$06 \quad 1644$

$06 \quad 1644$

$06 \quad 1645$

$06 \quad 1645$

$06 \quad 1646$

$06 \quad 1646$

$06 \quad 1647$

$06 \quad 1647$

$06 \quad 1648$

061649

061649

$20 \quad 1545$

$20 \quad 1545$

$20 \quad 1546$

$20 \quad 1546$

$20 \quad 1547$

$20 \quad 1547$

$20 \quad 1548$

201549

$20 \quad 1549$

$20 \quad 1550$

$\begin{array}{ccc}0.2 & 700 & 531 \\ 2.8 & 700 & 532 \\ 5.0 & 700 & 524 \\ 9.8 & 700 & 531 \\ 15 & 700 & 532 \\ 20 & 700 & 532 \\ 25 & 700 & 532 \\ 30 & 700 & 533 \\ 35 & 700 & 530 \\ 40 & 700 & 532 \\ 45 & 700 & 530 \\ & & \\ 0.2 & 900 & 531 \\ 2.8 & 900 & 529 \\ 4.9 & 900 & 530 \\ 9.7 & 900 & 531 \\ 15 & 900 & 529 \\ 20 & 900 & 533 \\ 25 & 900 & 529 \\ 30 & 900 & 532 \\ 35 & 900 & 528 \\ 40 & 900 & 532 \\ 45 & 900 & 533 \\ & & \\ 0.5 & 200 & 646 \\ 2.8 & 200 & 646 \\ 4.9 & 200 & 646 \\ 10 & 200 & 647 \\ 15 & 200 & 647 \\ 20 & 200 & 647 \\ 25 & 200 & 646 \\ 30 & 200 & 645 \\ 35 & 200 & 649 \\ 40 & 200 & 652\end{array}$

$\begin{array}{ll}7.7 & 29.6 \\ 7.8 & 29.2 \\ 7.7 & 28.2 \\ 7.4 & 27.8 \\ 7.4 & 27.6 \\ 7.4 & 27.5 \\ 7.4 & 27.5 \\ 7.4 & 27.5 \\ 7.4 & 27.5 \\ 7.4 & 27.5 \\ 7.4 & 27.5 \\ 7.7 & 29.7 \\ 7.7 & 29.3 \\ 7.7 & 28.7 \\ 7.4 & 27.7 \\ 7.4 & 27.6 \\ 7.4 & 27.6 \\ 7.4 & 27.6 \\ 7.4 & 27.5 \\ 7.4 & 27.6 \\ 7.4 & 27.5 \\ 7.4 & 27.5 \\ 7.5 & 24.0 \\ 7.4 & 24.0 \\ 7.4 & 24.0 \\ 7.4 & 24.0 \\ 7.4 & 24.0 \\ 7.4 & 24.0 \\ 7.4 & 24.0 \\ 7.4 & 24.0 \\ 7.4 & 24.0 \\ 7.4 & 24.0 \\ & \\ 7 & \end{array}$

\begin{tabular}{|c|c|}
\hline$\cdots$ & \\
\hline$\cdots$ & \\
\hline$\cdots$ & \\
\hline- & \\
\hline$\cdots$ & \\
\hline$\cdots$ & \\
\hline$\cdots$ & \\
\hline$\cdots$ & \\
\hline$\cdots$ & \\
\hline$\cdots$ & \\
\hline-- & \\
\hline- & \\
\hline-- & \\
\hline$\cdots$ & \\
\hline- & \\
\hline$\cdots$ & \\
\hline - - & \\
\hline$\cdots$ & \\
\hline$\cdots$ & \\
\hline$\cdots$ & \\
\hline$\ldots$ & \\
\hline & \\
\hline-- & \\
\hline$-\cdots$ & \\
\hline$\cdots$ & \\
\hline$\cdots$ & \\
\hline$\cdots$ & \\
\hline$\cdots$ & \\
\hline- & \\
\hline$\ldots$ & \\
\hline - & \\
\hline
\end{tabular}

99

103

77

72

69

67

65

66

66

65

63

94

96

93

73

69

67

66

65

65

65

65

64

63

63

63

62

62

63

63

63

61 
Table 30. Water-quality data for station 390721081443001, Ohio River at river mile 203.6, June to October, 1995, Continued.

$\left[\mathrm{ft}=\right.$ feet $; \mu \mathrm{S} / \mathrm{cm}=$ microsiemens per centımeter $;{ }^{\circ} \mathrm{C}=$ degrees Celsius: $\mathrm{mg} / \mathrm{L}=\mathrm{milligrams}$ per liter: - = data not collected]

\begin{tabular}{|c|c|c|c|c|c|c|c|c|c|}
\hline Date & Time & $\begin{array}{l}\text { Sampling } \\
\text { depth } \\
(\mathrm{ft})\end{array}$ & $\begin{array}{c}\text { Sample } \\
\text { location } \\
\text { (ft Erom } \\
\text { left bank) }\end{array}$ & $\begin{array}{l}\text { Specific } \\
\text { conduct - } \\
\text { ance } \\
(\mu \mathrm{S} / \mathrm{cm})\end{array}$ & $\begin{array}{l}\mathrm{pH} \\
\text { (stan- } \\
\text { dard } \\
\text { units) }\end{array}$ & $\begin{array}{c}\text { Temper- } \\
\text { ature, } \\
\text { water } \\
\left({ }^{\circ} \mathrm{C}\right)\end{array}$ & $\begin{array}{c}\text { Trans- } \\
\text { parency } \\
\text { (Secchl } \\
\text { dlsk) } \\
\text { (tt) }\end{array}$ & $\begin{array}{l}\text { Dissolved } \\
\text { oxygen } \\
(\mathrm{mg} / \mathrm{L})\end{array}$ & $\begin{array}{c}\text { Dissolved } \\
\text { oxygen } \\
\text { (percent } \\
\text { satura- } \\
\text { tion) }\end{array}$ \\
\hline
\end{tabular}

\begin{tabular}{|c|c|c|c|}
\hline \multicolumn{4}{|c|}{ September } \\
\hline 20 & 1522 & 0.7 & 700 \\
\hline 20 & 1522 & 3.3 & 700 \\
\hline 20 & 1523 & 4.9 & 700 \\
\hline 20 & 1524 & 10 & 700 \\
\hline 20 & 1524 & 15 & 70 \\
\hline 20 & 1525 & 20 & 70 \\
\hline 20 & 1525 & 25 & \\
\hline 20 & 1526 & 30 & \\
\hline 20 & 1527 & 35 & \\
\hline 20 & 1527 & 40 & \\
\hline 20 & 1528 & 45 & \\
\hline 20 & 1530 & 0.5 & \\
\hline 20 & 1530 & 3.3 & 700 \\
\hline 20 & 1531 & 5.0 & 7 \\
\hline 20 & 1531 & 11 & 700 \\
\hline 20 & 1532 & 15 & 700 \\
\hline 20 & 1532 & 20 & 70 \\
\hline 20 & 1533 & 25 & 700 \\
\hline 20 & 1533 & 30 & 706 \\
\hline 20 & 1534 & 35 & 70 \\
\hline 20 & 1534 & 40 & \\
\hline 20 & 1535 & 42 & \\
\hline 20 & 1513 & 1.0 & 90 \\
\hline 20 & 1514 & 3.2 & 9 \\
\hline 20 & 1514 & 5.0 & 9 \\
\hline 20 & 1515 & 9.9 & 9 \\
\hline 20 & 1515 & 15 & \\
\hline 20 & 1516 & 21 & 9 \\
\hline 20 & 1517 & 25 & \\
\hline 20 & 1517 & 30 & \\
\hline 20 & 1518 & 36 & \\
\hline 20 & 1518 & 40 & \\
\hline 20 & 1519 & 46 & \\
\hline
\end{tabular}

\begin{tabular}{|c|c|c|c|c|c|}
\hline 647 & 7.4 & 24.0 & $\cdots$ & 5.3 & 64 \\
\hline 647 & 7.4 & 24.0 & - & 5.3 & \\
\hline 648 & 7.4 & 24.0 & $\cdots$ & 5.3 & \\
\hline 647 & 7.4 & 24.0 & $\cdots$ & 5.2 & \\
\hline 649 & 7.4 & 24.0 & $\cdots$ & 5.2 & \\
\hline 645 & 7.4 & 24.0 & $\cdots$ & 5.2 & \\
\hline 649 & 7.4 & 24.0 & $\ldots$ & 5.2 & \\
\hline 648 & 7.4 & 24.0 & $\ldots$ & 5.2 & \\
\hline 647 & 7.4 & 24.0 & $\cdots$ & 5.2 & \\
\hline 648 & 7.4 & 24.0 & $\ldots$ & 5.2 & \\
\hline 648 & 7.4 & 24.0 & $\cdots$ & 5.2 & \\
\hline 647 & 7.4 & 24.0 & $\cdots$ & 5.2 & \\
\hline 647 & 7.4 & 24.0 & $\cdots$ & 5.2 & \\
\hline 648 & 7.4 & 24.0 & $\cdots$ & 5.2 & \\
\hline 648 & 7.4 & 24.0 & $\cdots$ & 5.2 & \\
\hline 648 & 7.4 & 24.0 & $\cdots$ & 5.2 & \\
\hline 647 & 7.4 & 24.0 & $\cdots$ & 5.2 & \\
\hline 648 & 7.4 & 24.0 & $\cdots$ & 5.2 & \\
\hline 648 & 7.4 & 24.0 & $\cdots$ & 5.2 & \\
\hline 648 & 7.4 & 24.0 & $\cdots$ & 5.2 & \\
\hline 648 & 7.4 & 24.0 & - & 5.2 & \\
\hline 648 & 7.4 & 24.0 & $\cdots$ & 5.2 & \\
\hline 645 & 7.4 & 24.0 & $\cdots$ & 5.2 & \\
\hline 642 & 7.4 & 24.0 & $\cdots$ & 5.2 & \\
\hline 644 & 7.4 & 24.0 & $\cdots$ & 5.2 & \\
\hline 647 & 7.4 & 24.0 & $\cdots$ & 5.1 & \\
\hline 648 & 7.4 & 24.0 & - & 5.2 & \\
\hline 649 & 7.4 & 24.0 & $\cdots$ & 5.2 & \\
\hline 642 & 7.4 & 24.0 & - & 5.2 & \\
\hline 648 & 7.4 & 24.0 & $\cdots$ & 5.2 & \\
\hline 642 & 7.4 & 24.0 & $\cdots$ & 5.2 & \\
\hline 645 & 7.4 & 24.0 & $\cdots$ & 5.2 & \\
\hline 643 & 7.4 & 24.0 & $\cdots$ & 5.2 & \\
\hline
\end{tabular}


Table 30. Water-quality data for station 390721081443001, Ohio River at river mile 203.6, June to october, 1995, Continued.

[ft $=$ feet; $\mu \mathrm{S} / \mathrm{Cm}=$ microsiemens per centimeter $;{ }^{\circ} \mathrm{C}=$ degrees Celsius; $\mathrm{mg} / \mathrm{L}=\mathrm{milligrams}$ per liter: . - = data not collected

\begin{tabular}{|c|c|c|c|c|c|c|c|c|c|}
\hline Date & Time & $\begin{array}{l}\text { Sampling } \\
\text { depth } \\
\text { (ft) }\end{array}$ & $\begin{array}{l}\text { Sample } \\
\text { location } \\
\text { (ft from } \\
\text { left bank) }\end{array}$ & $\begin{array}{l}\text { Specific } \\
\text { conduct - } \\
\text { ance } \\
(\mu \mathrm{s} / \mathrm{cm})\end{array}$ & $\begin{array}{l}\mathrm{pH} \\
\text { (stan- } \\
\text { dard } \\
\text { units) }\end{array}$ & $\begin{array}{c}\text { Temper- } \\
\text { ature, } \\
\text { water } \\
\left({ }^{\circ} \mathrm{C}\right)\end{array}$ & $\begin{array}{l}\text { Trans- } \\
\text { parency } \\
\text { (Secchi } \\
\text { disk) } \\
(E t)\end{array}$ & $\begin{array}{l}\text { Dissolved } \\
\text { oxygen } \\
(\mathrm{mg} / \mathrm{L})\end{array}$ & $\begin{array}{c}\text { Dissolved } \\
\text { oxygen } \\
\text { (percent } \\
\text { satura- } \\
\text { tion) }\end{array}$ \\
\hline
\end{tabular}

$\begin{array}{cccc}\text { October } & & \\ 05 & 1337 & 0.3 & 200 \\ 05 & 1336 & 3.0 & 200 \\ 05 & 1335 & 5.3 & 200 \\ 05 & 1335 & 10 & 200 \\ 05 & 1334 & 15 & 200 \\ 05 & 1334 & 20 & 200 \\ 05 & 1333 & 25 & 200 \\ 05 & 1332 & 30 & 200 \\ 05 & 1332 & 38 & 200 \\ & & & \\ 05 & 1330 & 0.3 & 500 \\ 05 & 1330 & 3.1 & 500 \\ 05 & 1329 & 5.1 & 500 \\ 05 & 1329 & 10 & 500 \\ 05 & 1328 & 15 & 500 \\ 05 & 1328 & 20 & 500 \\ 05 & 1327 & 25 & 500 \\ 05 & 1327 & 31 & 500 \\ 05 & 1326 & 35 & 500 \\ 05 & 1326 & 39 & 500 \\ 05 & 1324 & 0.3 & 700 \\ 05 & 1322 & 3.7 & 700 \\ 05 & 1323 & 5.0 & 700 \\ 05 & 1321 & 10 & 700 \\ 05 & 1321 & 15 & 700 \\ 05 & 1320 & 18 & 700 \\ 05 & 1319 & 25 & 700 \\ 05 & 1319 & 30 & 700 \\ 05 & 1318 & 35 & 700 \\ 05 & 1317 & 41 & 700 \\ 05 & 1317 & 45 & \end{array}$

645
647
651
646
654
651
654
656
666
646
646
650
655
659
653
653
659
650
659
641
647
649
652
656
653
658
658
655
659
662
6

\section{3}

7.3

7.3

7.3

7.3

7.3

7.3

7.3

7.3

7.3

7.3

7.3

7.3

7.3

7.3

7.3

7.3

7.3

7.3

7.3

7.3

7.3

7.3

7.3

7.3

7.3

7.3

7.3

7.3

7.3

21.9
21.9
21.9
21.8
21.8
21.8
21.8
21.8
21.8
21.9
21.9
21.9
21.8
21.8
21.8
21.8
21.8
21.8
21.8

21.9
21.9
21.9
21.9
21.9
21.8
21.8
21.8
21.8
21.8
21.8

- 6.4

74

- 6.4

- 6.4

. 6.4

.. 6.4

.. 6.4

. 6.4

. 6.4

.. 6.4

. 6.5

. 6.6

. 6.5

-.5
$\ldots$

. 6.5

. 6.5

- 6.4

. 6.4

- 6.4

. 6.4

75
74

74
74

74

74

74

75

662

.. 6.6

6.6
$-\quad 6.7$

. 6.6

6.6
$\ldots \quad 6$

. 6.5

. 6.5

. 6.5

- 6.5

.. 6.5

- 6.5

. 6.5

76

77
75

75

75
75

75

74

75

75
74

75

76

77

76

76
76

75

75
75

75
75

75
75

75

75
75

75 
Table 30. Water-quality data for station 390721081443001, Ohio River at river mile 203.6, June to October, 1995, Continued.

$\left[\mathrm{ft}=\mathrm{feet} ; \mu \mathrm{S} / \mathrm{cm}=\right.$ microsiemens per centimeter $;{ }^{\circ} \mathrm{C}=$ degrees Celsius; $\mathrm{mg} / \mathrm{L}=$ miligrams per liter: . = data not collected]

\begin{tabular}{|c|c|c|c|c|c|c|c|c|c|}
\hline Date & Time & $\begin{array}{l}\text { Sampling } \\
\text { depth } \\
\text { (ft) }\end{array}$ & $\begin{array}{l}\text { Sample } \\
\text { location } \\
\text { (ft from } \\
\text { lett bank) }\end{array}$ & $\begin{array}{l}\text { Specific } \\
\text { conduct- } \\
\text { ance } \\
(\mu \mathrm{s} / \mathrm{cm})\end{array}$ & $\begin{array}{l}\mathrm{pH} \\
\text { (stan- } \\
\text { dard } \\
\text { units) }\end{array}$ & $\begin{array}{l}\text { Temper- } \\
\text { ature, } \\
\text { water } \\
\left({ }^{\circ} \mathrm{C}\right)\end{array}$ & $\begin{array}{l}\text { Trans - } \\
\text { parency } \\
\text { (secchl } \\
\text { disk) } \\
\text { (It) }\end{array}$ & $\begin{array}{l}\text { Dissolved } \\
\text { oxygen } \\
(\mathrm{mg} / \mathrm{L})\end{array}$ & $\begin{array}{c}\text { Dissolved } \\
\text { oxygen } \\
\text { (percent } \\
\text { satura. } \\
\text { tron) }\end{array}$ \\
\hline
\end{tabular}

\begin{tabular}{|c|c|c|c|c|c|c|c|c|c|}
\hline \multicolumn{10}{|c|}{ October } \\
\hline 05 & 1307 & 0.6 & 900 & 640 & 7.4 & 21.9 & $\cdots$ & 6.8 & 79 \\
\hline 05 & 1307 & 2.9 & 900 & 648 & 7.3 & 21.9 & $\cdots$ & 6.7 & 77 \\
\hline 05 & 1308 & 4.8 & 900 & 646 & 7.3 & 22.0 & $\cdots$ & 6.7 & 78 \\
\hline 05 & 1308 & 10 & 900 & 655 & 7.3 & 21.9 & $\cdots$ & 6.6 & 77 \\
\hline 05 & 1309 & 15 & 900 & 650 & 7.3 & 21.9 & $\cdots$ & 6.6 & 76 \\
\hline 05 & 1310 & 20 & 900 & 655 & 7.3 & 21.9 & $\cdots$ & 6.5 & 76 \\
\hline 05 & 1310 & 25 & 900 & 654 & 7.3 & 21.9 & 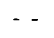 & 6.6 & 76 \\
\hline 05 & 1311 & 30 & 900 & 650 & 7.3 & 21.9 & $\cdots$ & 6.5 & 75 \\
\hline 05 & 1311 & 35 & 900 & 656 & 7.3 & 21.9 & - & 6.4 & 74 \\
\hline 05 & 1312 & 40 & 900 & 660 & 7.3 & 21.9 & - & 6.3 & 74 \\
\hline 05 & 1312 & 45 & 900 & 653 & 7.3 & 21.9 & $\cdots$ & 6.5 & 75 \\
\hline 05 & 1313 & 49 & 900 & 653 & 7.3 & 21.9 & $\cdots$ & 6.4 & 74 \\
\hline 19 & 1638 & 0.9 & 200 & 517 & 7.5 & 19.3 & $\cdots$ & 7.8 & 86 \\
\hline 19 & 1638 & 3.1 & 200 & 516 & 7.5 & 19.2 & $\cdots$ & 7.7 & 84 \\
\hline 19 & 1639 & 5.1 & 200 & 516 & 7.5 & 18.8 & $\cdots$ & 7.4 & 80 \\
\hline 19 & 1639 & 9.7 & 200 & 514 & 7.4 & 18.5 & $\cdots$ & 7.2 & 77 \\
\hline 19 & 1640 & 15 & 200 & 513 & 7.4 & 18.2 & - & 7.1 & 76 \\
\hline 19 & 1640 & 21 & 200 & 513 & 7.4 & 18.2 & $\cdots$ & 7.1 & 76 \\
\hline 19 & 1641 & 25 & 200 & 514 & 7.4 & 18.2 & - & 7.0 & 75 \\
\hline 19 & 1641 & 29 & 200 & 514 & 7.4 & 18.1 & $\cdots$ & 6.9 & 74 \\
\hline 19 & 1642 & 35 & 200 & 514 & 7.4 & 18.1 & $\cdots$ & 6.9 & 74 \\
\hline 19 & 1644 & 0.9 & 500 & 515 & 7.5 & 19.2 & $\cdots$ & 7.7 & 85 \\
\hline 19 & 1644 & 3.2 & 500 & 515 & 7.5 & 19.0 & - & 7.4 & 80 \\
\hline 19 & 1645 & 5.2 & 500 & 514 & 7.4 & 18.5 & $\cdots$ & 7.3 & 78 \\
\hline 19 & 1645 & 9.9 & 500 & 513 & 7.4 & 18.2 & $\cdots$ & 7.1 & 76 \\
\hline 19 & 1646 & 15 & 500 & 513 & 7.4 & 18.2 & - & 7.1 & 76 \\
\hline 19 & 1646 & 20 & 500 & 513 & 7.4 & 18.2 & $\cdots$ & 7.0 & 75 \\
\hline 19 & 1647 & 25 & 500 & 514 & 7.4 & 18.2 & $\cdots$ & 7.0 & 74 \\
\hline 19 & 1647 & 30 & 500 & 514 & 7.4 & 18.2 & - & 6.9 & 74 \\
\hline 19 & 1648 & 35 & 500 & 515 & 7.4 & 18.2 & $\cdots$ & 6.9 & 74 \\
\hline 19 & 1648 & 39 & 500 & 514 & 7.4 & 18.2 & $\cdots$ & 6.9 & 74 \\
\hline
\end{tabular}


Table 30. Water-quality data for station 390721081443001, Ohio River at river mile 203.6, June to October, 1995, Continued.

[ft $=$ feet; $\mu \mathrm{s} / \mathrm{cm}=$ microsiemens per centimeter ${ }^{\circ} \mathrm{C}=$ degrees Celsius;

$\mathrm{mg} / \mathrm{L}=$ milligrams per liter; .. = data not collected]

\begin{tabular}{|c|c|c|c|c|c|c|c|c|c|}
\hline Date & Time & $\begin{array}{l}\text { Sampling } \\
\text { depth } \\
\text { (ft) }\end{array}$ & $\begin{array}{c}\text { Sample } \\
\text { location } \\
\text { (tt from } \\
\text { left bank) }\end{array}$ & $\begin{array}{l}\text { Specific } \\
\text { conduct- } \\
\text { ance } \\
(\mu \mathrm{S} / \mathrm{cm})\end{array}$ & $\begin{array}{l}\mathrm{pH} \\
\text { (stan- } \\
\text { dard } \\
\text { units) }\end{array}$ & $\begin{array}{c}\text { Temper- } \\
\text { ature, } \\
\text { water } \\
\left({ }^{\circ} \mathrm{C}\right)\end{array}$ & $\begin{array}{c}\text { Trans- } \\
\text { parency } \\
\text { (Secchi } \\
\text { disk) } \\
(t t)\end{array}$ & $\begin{array}{l}\text { Dissolved } \\
\text { oxygen } \\
(\mathrm{mg} / \mathrm{L})\end{array}$ & $\begin{array}{c}\text { Dissolved } \\
\text { oxygen } \\
\text { (percent } \\
\text { satura- } \\
\text { tion) }\end{array}$ \\
\hline
\end{tabular}

\section{October}

\begin{tabular}{|c|c|c|c|c|c|c|c|c|}
\hline & & & & & & & & \\
\hline $19 \quad 1650$ & 1.1 & 700 & 516 & 7.5 & 19.0 & - & 7.5 & 82 \\
\hline 1650 & 3.2 & 700 & 515 & 7.4 & 18.7 & -- & 7.3 & 79 \\
\hline 1651 & 5.1 & 700 & 514 & 7.4 & 18.5 & $\cdots$ & 7.2 & 77 \\
\hline 1651 & 9.6 & 700 & 514 & 7.4 & 18.2 & $\cdots$ & 7.1 & 76 \\
\hline 1652 & 15 & 700 & 514 & 7.4 & 18.2 & $\cdots$ & 7.1 & 76 \\
\hline 1653 & 20 & 700 & 514 & 7.4 & 18.2 & $\cdots$ & 7.0 & 75 \\
\hline 1652 & 25 & 700 & 514 & 7.4 & 18.2 & $\cdots$ & 7.0 & 75 \\
\hline 1655 & 30 & 700 & 514 & 7.4 & 18.2 & $\cdots$ & 6.9 & 74 \\
\hline 1654 & 36 & 700 & 514 & 7.4 & 18.2 & $\cdots$ & 6.9 & 74 \\
\hline 1654 & 40 & 700 & 514 & 7.4 & 18.2 & $\cdots$ & 6.9 & 74 \\
\hline 1653 & 45 & 700 & 514 & 7.4 & 18.2 & $\cdots$ & 6.9 & 74 \\
\hline 1658 & 1.1 & 900 & 514 & 7.4 & 18.5 & $\cdots$ & 7.1 & 77 \\
\hline 1658 & 3.2 & 900 & 513 & 7.4 & 18.5 & -- & 7.1 & 77 \\
\hline 1659 & 5.1 & 900 & 513 & 7.4 & 18.4 & $\cdots$ & 7.1 & 76 \\
\hline 1659 & 9.9 & 900 & 514 & 7.4 & 18.3 & $\cdots$ & 6.9 & 74 \\
\hline 1700 & 15 & 900 & 515 & 7.4 & 18.3 & $\cdots$ & 7.0 & 75 \\
\hline 1700 & 20 & 900 & 514 & 7.4 & 18.4 & $\cdots$ & 7.0 & 75 \\
\hline 1701 & 25 & 900 & 514 & 7.4 & 18.4 & $\cdots$ & 7.0 & 76 \\
\hline 1701 & 30 & 900 & 513 & 7.4 & 18.4 & $\cdots$ & 6.8 & 73 \\
\hline 1702 & 35 & 900 & 516 & 7.4 & 18.3 & $\cdots$ & 6.8 & 73 \\
\hline 1702 & 40 & 900 & 513 & 7.4 & 18.3 & $\cdots$ & 6.8 & 73 \\
\hline 1703 & 45 & 900 & 517 & 7.4 & 18.3 & $\cdots$ & 6.7 & 72 \\
\hline 1703 & 50 & 900 & 521 & 7.4 & 18.3 & $\cdots$ & 6.9 & 74 \\
\hline
\end{tabular}


Table 31. Daily maximum, minimum, and mean specific conductance at station 392145081185601, from the Willow Island Dam (upstream) continuous-recording water-quality monitor, June to October 1995.

$[\cdots$, value not determined]

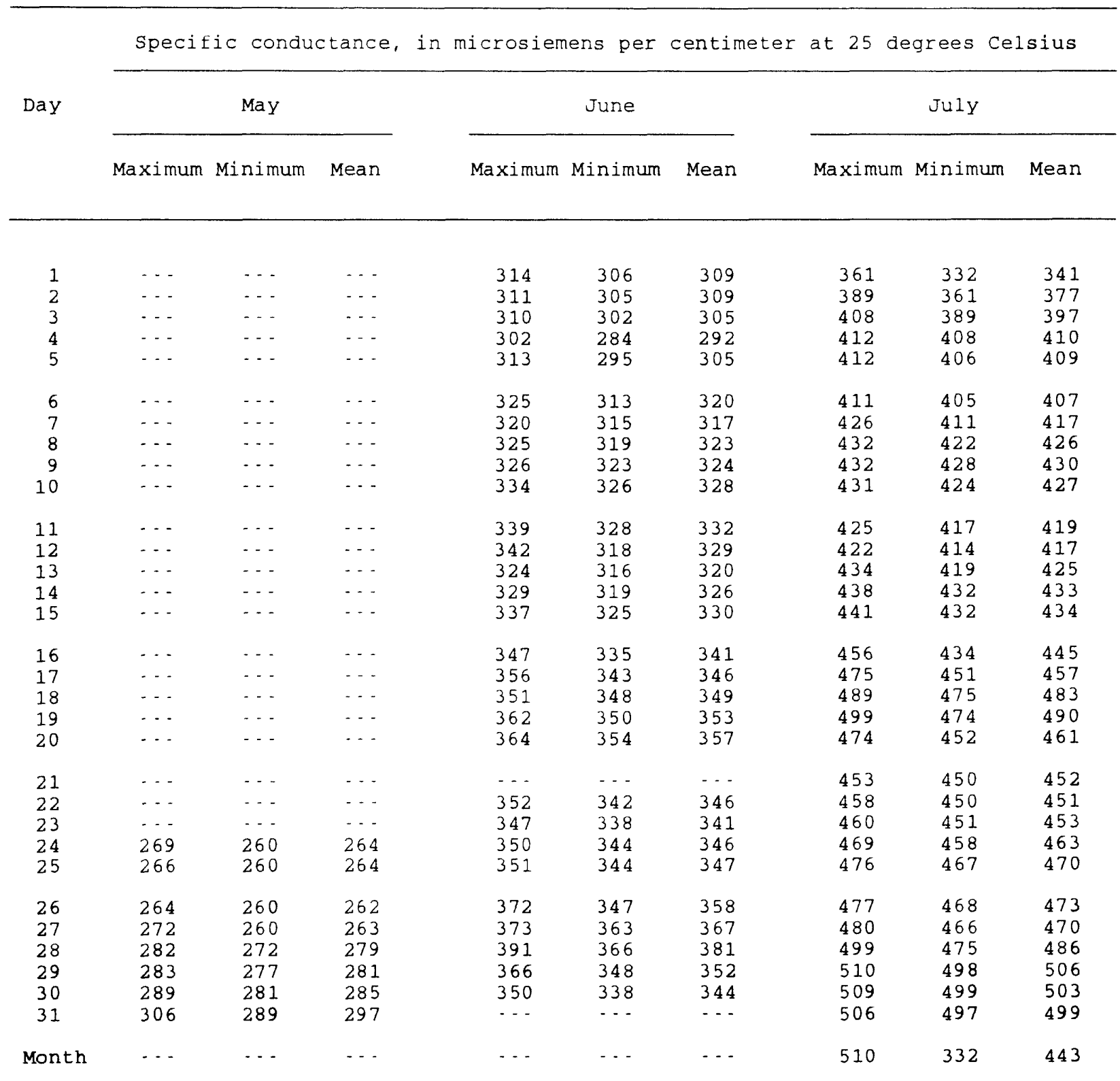


Table 31. Daily maximum, minimum, and mean specific conductance at station 392145081185601, from the Willow Island Dam (upstream) continuous-recording water-quality monitor, June to october 1995-continued.

[.., value not determined]

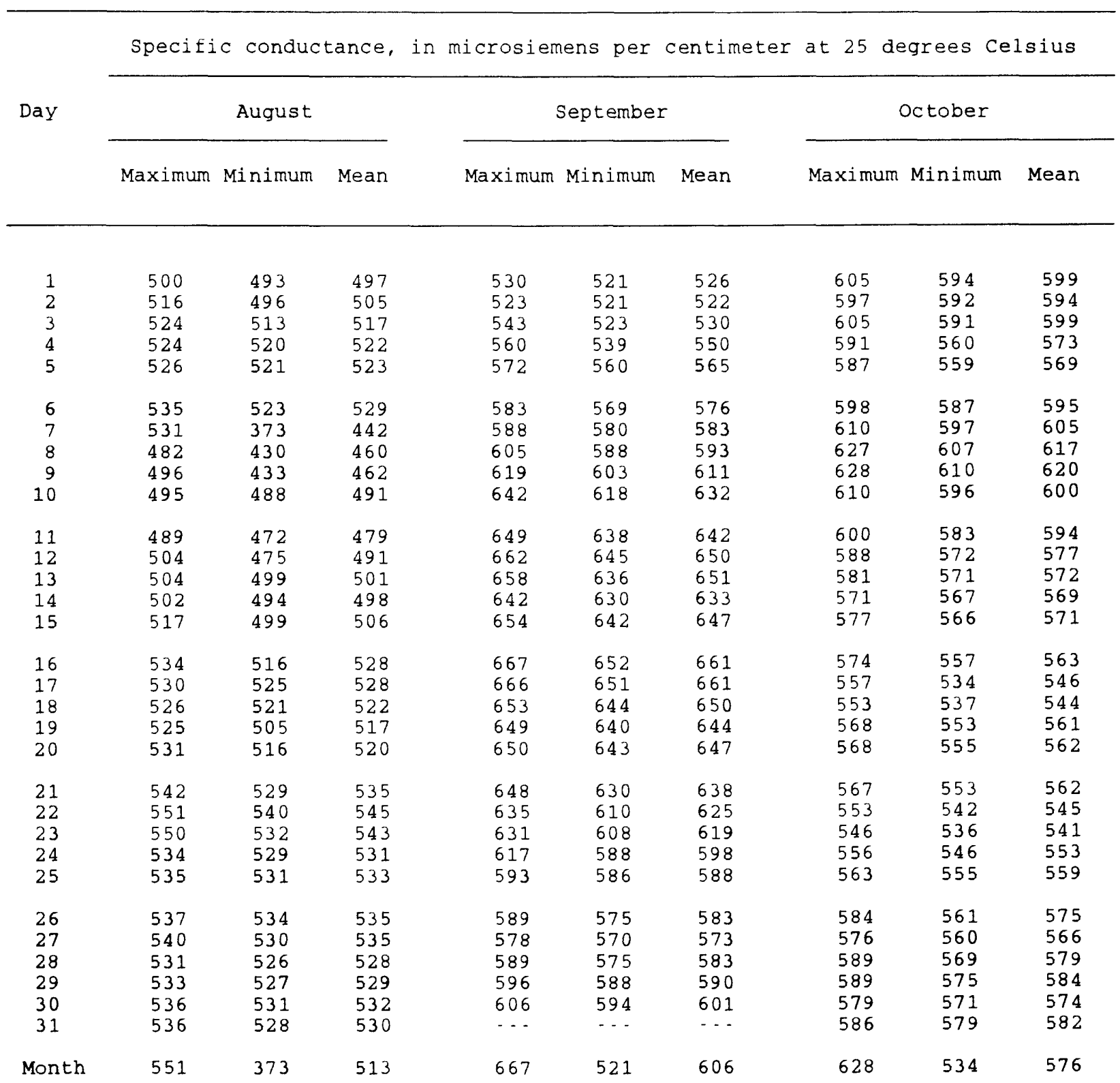


Table 32. Daily maximum, minimum, and median $p H$ at station 392145081185601, from the Willow Island Dam (upstream) continuous-recording water-quality monitor, June to October 1995.

$[\cdots$, value not determined]

\begin{tabular}{|c|c|c|c|c|c|c|c|c|c|}
\hline \multirow[t]{2}{*}{ Day } & \multicolumn{3}{|c|}{ May } & \multicolumn{3}{|c|}{ June } & \multicolumn{3}{|c|}{ Ju1y } \\
\hline & Maximum & Minimum & Median & Maximum & Minimum & Median & Maximum & Minimum & Median \\
\hline $\begin{array}{l}1 \\
2 \\
3 \\
4 \\
5\end{array}$ & $\begin{array}{l}\cdots \\
\cdots \\
\cdots \\
\cdots \\
\cdots\end{array}$ & $\begin{array}{l}\cdots \\
\cdots \\
\cdots \\
- \\
\cdots \\
-\end{array}$ & $\begin{array}{l}\cdots \\
\cdots \\
\cdots \\
\cdots \\
\cdots\end{array}$ & $\begin{array}{l}7.6 \\
7.6 \\
7.6 \\
7.5 \\
7.7\end{array}$ & $\begin{array}{l}7.5 \\
7.4 \\
7.5 \\
7.4 \\
7.5\end{array}$ & $\begin{array}{l}7.5 \\
7.5 \\
7.5 \\
7.5 \\
7.5\end{array}$ & $\begin{array}{l}7.3 \\
7.4 \\
7.4 \\
7.4 \\
7.4\end{array}$ & $\begin{array}{l}7.1 \\
7.3 \\
7 \cdot 3 \\
7 \cdot 3 \\
7.3\end{array}$ & $\begin{array}{l}7.2 \\
7.3 \\
7.4 \\
7.4 \\
7.3\end{array}$ \\
\hline $\begin{array}{r}6 \\
7 \\
8 \\
9 \\
10\end{array}$ & $\begin{array}{l}\cdots \\
\cdots \\
\cdots \\
\cdots \\
\cdots\end{array}$ & $\begin{array}{l}\cdots \\
\cdots \\
\cdots \\
\cdots \\
\cdots\end{array}$ & $\begin{array}{l}\cdots \\
\cdots \\
\cdots \\
\cdots \\
-\end{array}$ & $\begin{array}{l}7.6 \\
7.8 \\
8.4 \\
8.3 \\
8.1\end{array}$ & $\begin{array}{l}7.5 \\
7.5 \\
7.6 \\
7.7 \\
7.7\end{array}$ & $\begin{array}{l}7.5 \\
7.6 \\
7.8 \\
7.8 \\
7.7\end{array}$ & $\begin{array}{l}7.4 \\
7.5 \\
7.6 \\
7.7 \\
7.7\end{array}$ & $\begin{array}{l}7.3 \\
7.4 \\
7.4 \\
7.4 \\
7.4\end{array}$ & $\begin{array}{l}7.4 \\
7.4 \\
7.4 \\
7.5 \\
7.6\end{array}$ \\
\hline $\begin{array}{l}11 \\
12 \\
13 \\
14 \\
15\end{array}$ & $\begin{array}{l}\cdots \\
\cdots \\
\cdots \\
\cdots \\
\cdots\end{array}$ & $\begin{array}{l}\cdots \\
\cdots \\
\cdots \\
\cdots\end{array}$ & $\begin{array}{l}\cdots \\
\cdots \\
\cdots \\
\cdots \\
\cdots\end{array}$ & $\begin{array}{l}8.0 \\
7.5 \\
7.3 \\
7.5 \\
7.6\end{array}$ & $\begin{array}{l}7.5 \\
7.3 \\
7.2 \\
7.3 \\
7.3\end{array}$ & $\begin{array}{l}7.7 \\
7.3 \\
7.3 \\
7.3 \\
7.4\end{array}$ & $\begin{array}{l}7.7 \\
7.6 \\
7.7 \\
7.7 \\
7.6\end{array}$ & $\begin{array}{l}7.5 \\
7.3 \\
7.3 \\
7.4 \\
7.3\end{array}$ & $\begin{array}{l}7.6 \\
7.5 \\
7.4 \\
7.5 \\
7.4\end{array}$ \\
\hline $\begin{array}{l}16 \\
17 \\
18 \\
19 \\
20\end{array}$ & $\begin{array}{l}\cdots \\
\cdots \\
\cdots \\
\cdots \\
-\end{array}$ & $\begin{array}{l}\cdots \\
\cdots \\
\cdots \\
\cdots \\
-\end{array}$ & $\begin{array}{c}\cdots \\
\cdots \\
\cdots \\
\cdots \\
\cdots\end{array}$ & $\begin{array}{l}7.6 \\
7.8 \\
7.7 \\
7.8 \\
7.5\end{array}$ & $\begin{array}{l}7.4 \\
7.3 \\
7.4 \\
7.3 \\
7.3\end{array}$ & $\begin{array}{l}7.4 \\
7.4 \\
7.4 \\
7.4 \\
7.4\end{array}$ & $\begin{array}{l}7.6 \\
7.6 \\
7.5 \\
7.5 \\
7.5\end{array}$ & $\begin{array}{l}7.3 \\
7 \cdot 3 \\
7 \cdot 3 \\
7 \cdot 3 \\
7.3\end{array}$ & $\begin{array}{l}7.4 \\
7.4 \\
7.3 \\
7.4 \\
7.4\end{array}$ \\
\hline $\begin{array}{l}21 \\
22 \\
23 \\
24 \\
25\end{array}$ & $\begin{array}{l}\ldots \\
\cdots \\
\cdots \\
7.4 \\
7.4\end{array}$ & $\begin{array}{l}\ldots \\
\cdots \\
7.3 \\
7.4\end{array}$ & $\begin{array}{l}\ldots \\
\ldots \\
\ldots . \\
7.4 \\
7.4\end{array}$ & $\begin{array}{l}7.6 \\
7.4 \\
7.4 \\
7.3\end{array}$ & $\begin{array}{l}7.3 \\
7.2 \\
7.2 \\
7.2\end{array}$ & $\begin{array}{l}-.- \\
7.4 \\
7.3 \\
7.3 \\
7.3\end{array}$ & $\begin{array}{l}7.6 \\
7.3 \\
7.3 \\
7.4 \\
7.4\end{array}$ & $\begin{array}{l}7.3 \\
7.2 \\
7.3 \\
7.2 \\
7.2\end{array}$ & $\begin{array}{l}7.4 \\
7 \cdot 3 \\
7 \cdot 3 \\
7 \cdot 3 \\
7.3\end{array}$ \\
\hline $\begin{array}{l}26 \\
27 \\
28 \\
29 \\
30 \\
31\end{array}$ & $\begin{array}{l}7.4 \\
7.4 \\
7.4 \\
7.5 \\
7.5 \\
7.6\end{array}$ & $\begin{array}{l}7.4 \\
7.3 \\
7.3 \\
7.4 \\
7.4 \\
7.4\end{array}$ & $\begin{array}{l}7.4 \\
7.4 \\
7.4 \\
7.4 \\
7.4 \\
7.5\end{array}$ & $\begin{array}{l}7.5 \\
7.4 \\
7.7 \\
7.4 \\
7.2 \\
\ldots .\end{array}$ & $\begin{array}{l}7.3 \\
7.3 \\
7.3 \\
7.2 \\
7.1 \\
\cdots .\end{array}$ & $\begin{array}{l}7.3 \\
7.3 \\
7.4 \\
7.3 \\
7.2 \\
-.-\end{array}$ & $\begin{array}{l}7.6 \\
7.7 \\
7.7 \\
7.5 \\
7.8 \\
7.6\end{array}$ & $\begin{array}{l}7.3 \\
7.4 \\
7.4 \\
7.4 \\
7.4 \\
7.4\end{array}$ & $\begin{array}{l}7.4 \\
7.5 \\
7.5 \\
7.4 \\
7.5 \\
7.4\end{array}$ \\
\hline Month & $\cdots$ & $\cdots$ & $\cdots$ & $\ldots$ & $\cdots$ & $\cdots$ & 7.8 & 7.1 & $\cdots$ \\
\hline
\end{tabular}


Table 32. Daily maximum, minimum, and median $p H$ at station 392145081185601, from the Willow Island Dam (upstream) continuous-recording water-quality monitor, June to October 1995-- Continued.

$[\cdots$, value not determined]

\begin{tabular}{|c|c|c|c|c|c|c|c|c|c|}
\hline \multirow[t]{2}{*}{ Day } & \multicolumn{3}{|c|}{ August } & \multicolumn{3}{|c|}{ September } & \multicolumn{3}{|c|}{ October } \\
\hline & Maximum & Minimum & Median & Maximum & Minimum & Median & Maximum & Minimum & Median \\
\hline 1 & 7.8 & 7.3 & 7.4 & 7.2 & 7.0 & 7.1 & 7.4 & 7.3 & 7.3 \\
\hline 2 & 7.5 & 7.3 & 7.4 & 7.3 & 7.1 & 7.1 & 7.3 & 7.2 & 7.3 \\
\hline 3 & 7.6 & 7.4 & 7.4 & 7.1 & 7.1 & 7.1 & 7.3 & 7.2 & 7.2 \\
\hline 4 & 7.5 & 7.3 & 7.4 & 7.1 & 7.0 & 7.1 & & $\cdots$ & $\cdots$ \\
\hline 5 & 7.4 & 7.3 & 7.4 & 7.4 & 7.1 & 7.2 & 7.5 & 7.4 & 7.4 \\
\hline 6 & 7.5 & 7.3 & 7.4 & 7.4 & 7.2 & 7.2 & 7.5 & 7.4 & 7.4 \\
\hline 7 & 7.3 & 7.1 & 7.2 & 7.3 & 7.2 & 7.2 & 7.4 & 7.4 & 7.4 \\
\hline 8 & 7.4 & 7.2 & 7.4 & 7.3 & 7.2 & 7.2 & 7.4 & 7.3 & 7.4 \\
\hline 9 & 7.5 & 7.4 & 7.4 & 7.3 & 7.2 & 7.2 & 7.4 & 7.3 & 7.4 \\
\hline 10 & 7.4 & 7.3 & 7.3 & 7.3 & 7.2 & 7.2 & 7.4 & 7.4 & 7.4 \\
\hline 11 & 7.4 & 7.2 & 7.3 & 7.3 & 7.2 & 7.2 & 7.4 & 7.4 & 7.4 \\
\hline 12 & 7.3 & 7.2 & 7.3 & 7.3 & 7.2 & 7.2 & 7.5 & 7.4 & 7.4 \\
\hline 13 & 7.5 & 7.2 & 7.3 & 7.3 & 7.2 & 7.2 & 7.5 & 7.4 & 7.4 \\
\hline 14 & 7.5 & 7.3 & 7.4 & 7.3 & 7.2 & 7.2 & 7.5 & 7.4 & 7.4 \\
\hline 15 & 7.7 & 7.3 & 7.4 & 7.4 & 7.2 & 7.3 & 7.4 & 7.3 & 7.3 \\
\hline 16 & 7.7 & 7.3 & 7.4 & 7.3 & 7.2 & 7.3 & 7.3 & 7.3 & 7.3 \\
\hline 17 & 7.7 & 7.2 & 7.4 & 7.3 & 7.2 & 7.3 & 7.4 & 7.3 & 7.3 \\
\hline 18 & 7.4 & 7.2 & 7.3 & 7.3 & 7.2 & 7.3 & 7.4 & 7.3 & 7.3 \\
\hline 19 & 7.5 & 7.2 & 7.3 & 7.5 & 7.3 & 7.3 & 7.4 & 7.4 & 7.4 \\
\hline 20 & 7.5 & 7.1 & 7.2 & 7.4 & 7.3 & 7.3 & 7.4 & 7.4 & 7.4 \\
\hline 21 & 7.4 & 7.1 & 7.2 & 7.4 & 7.3 & 7.3 & 7.4 & 7.4 & 7.4 \\
\hline 22 & 7.3 & 7.2 & 7.2 & 7.4 & 7.3 & 7.3 & 7.4 & 7.4 & 7.4 \\
\hline 23 & 7.6 & 7.2 & 7.3 & 7.4 & 7.3 & 7.3 & 7.5 & 7.4 & 7.5 \\
\hline 24 & 7.6 & 7.3 & 7.4 & 7.4 & 7.3 & 7.4 & 7.5 & 7.5 & 7.5 \\
\hline 25 & 7.5 & 7.3 & 7.4 & 7.5 & 7.4 & 7.4 & 7.5 & 7.5 & 7.5 \\
\hline 26 & 7.5 & 7.3 & 7.3 & 7.4 & 7.2 & 7.3 & 7.6 & 7.5 & 7.6 \\
\hline 27 & 7.4 & 7.3 & 7.3 & 7.3 & 7.2 & 7.3 & 7.6 & 7.6 & 7.6 \\
\hline 28 & 7.4 & 7.2 & 7.3 & 7.5 & 7.3 & 7.3 & 7.6 & 7.6 & 7.6 \\
\hline 29 & 7.3 & 7.2 & 7.2 & 7.5 & 7.2 & 7.3 & 7.6 & 7.6 & 7.6 \\
\hline 30 & 7.3 & 7.2 & 7.2 & 7.4 & 7.2 & 7.2 & 7.6 & 7.6 & 7.6 \\
\hline 31 & 7.2 & 7.1 & 7.1 & & - & $\cdots$ & 7.6 & 7.6 & 7.6 \\
\hline Month & 7.8 & 7.1 & $\cdots$ & 7.5 & 7.0 & $\cdots$ & $\cdots$ & $\cdots$ & $\cdots$ \\
\hline
\end{tabular}


Table 33. Daily maximum, minimum, and mean water temperature at station 392145081185601, from the Willow Island Dam (upstream) continuous-recording water-quality monitor, June to october 1995.

$[\cdots$, value not determined]

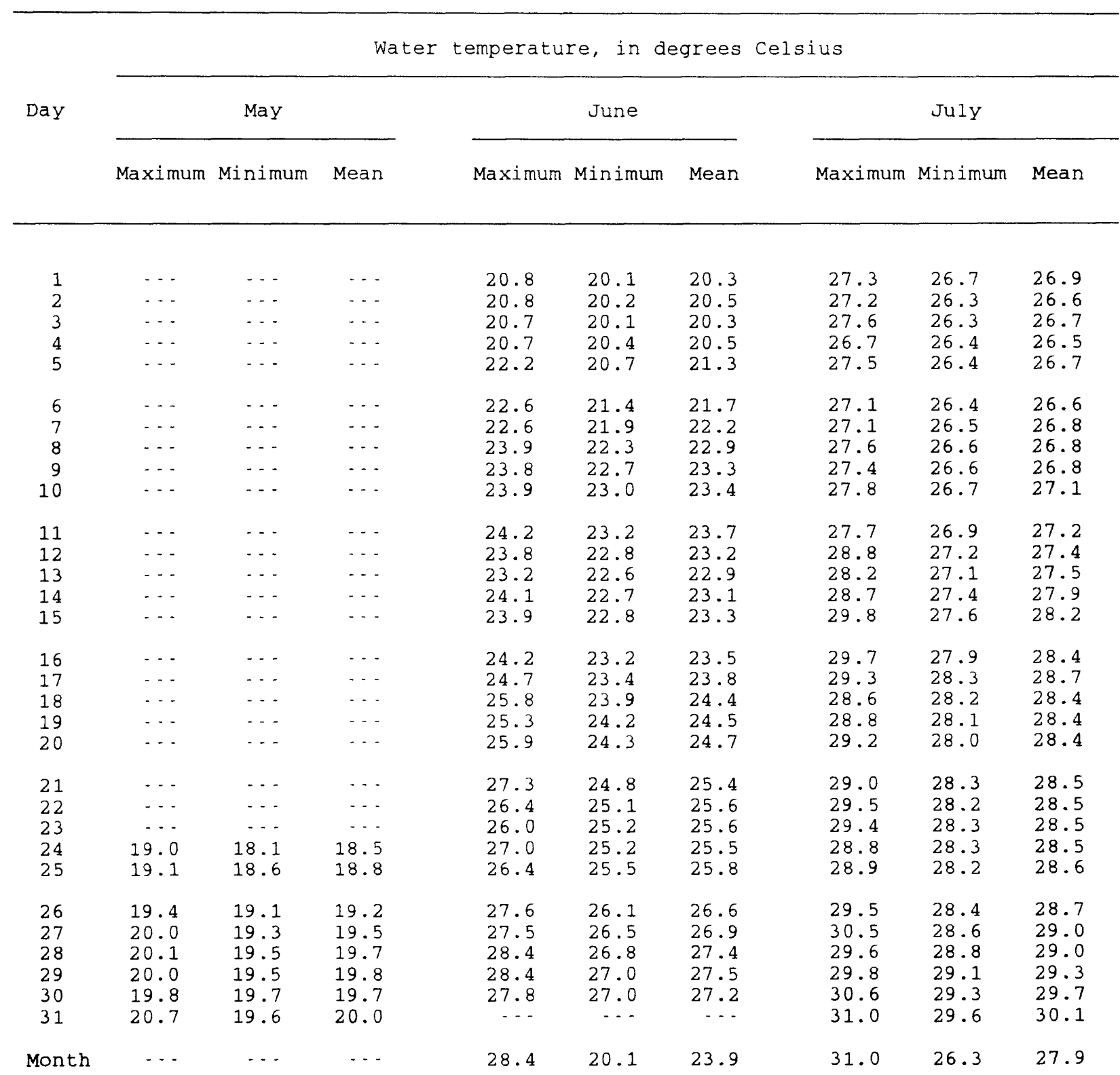


Table 33. Daily maximum, minimum, and mean water temperature at station 392145081185601, from the Willow Island Dam (upstream) continuous-recording water-quality monitor, June to October 1995-Continued.

$[\cdots$, value not determined]

\begin{tabular}{|c|c|c|c|c|c|c|c|c|c|}
\hline \multirow{3}{*}{ Day } & \multicolumn{6}{|c|}{ Water temperature, in degrees } & \multicolumn{3}{|c|}{ Celsius } \\
\hline & \multicolumn{3}{|c|}{ August } & \multicolumn{3}{|c|}{ September } & \multicolumn{3}{|c|}{ October } \\
\hline & Maximum & Minimum & Mean & Maximum & Minimum & Mean & Maximum & Minimum & Mean \\
\hline $\begin{array}{l}1 \\
2 \\
3 \\
4 \\
5\end{array}$ & $\begin{array}{l}30.8 \\
31.3 \\
30.8 \\
31.1 \\
30.8\end{array}$ & $\begin{array}{l}29.5 \\
29.7 \\
29.8 \\
30.2 \\
30.0\end{array}$ & $\begin{array}{l}29.9 \\
30.1 \\
30.4 \\
30.4 \\
30.3\end{array}$ & $\begin{array}{l}29.9 \\
29.2 \\
28.6 \\
28.6 \\
29.5\end{array}$ & $\begin{array}{l}29.2 \\
28.6 \\
28.2 \\
28.2 \\
28.3\end{array}$ & $\begin{array}{l}29.6 \\
28.8 \\
28.4 \\
28.3 \\
28.7\end{array}$ & $\begin{array}{l}22.3 \\
22.3 \\
22.7 \\
22.2 \\
22.1\end{array}$ & $\begin{array}{l}22.0 \\
22.0 \\
22.0 \\
22.1 \\
21.9\end{array}$ & $\begin{array}{l}22.1 \\
22.2 \\
22.2 \\
22.2 \\
22.0\end{array}$ \\
\hline $\begin{array}{r}6 \\
7 \\
8 \\
9 \\
10\end{array}$ & $\begin{array}{l}30.2 \\
29.8 \\
29.5 \\
29.7 \\
29.9\end{array}$ & $\begin{array}{l}29.5 \\
27.3 \\
28.7 \\
28.9 \\
29.2\end{array}$ & $\begin{array}{l}29.7 \\
28.4 \\
29.2 \\
29.2 \\
29.4\end{array}$ & $\begin{array}{l}29.1 \\
28.8 \\
28.5 \\
28.1 \\
27.6\end{array}$ & $\begin{array}{l}27.9 \\
27.7 \\
27.8 \\
27.4 \\
26.8\end{array}$ & $\begin{array}{l}28.4 \\
28.0 \\
27.9 \\
27.6 \\
27.2\end{array}$ & $\begin{array}{l}22.1 \\
22.0 \\
21.8 \\
21.8 \\
21.8\end{array}$ & $\begin{array}{l}21.8 \\
21.7 \\
21.5 \\
21.2 \\
21.2\end{array}$ & $\begin{array}{l}21.9 \\
21.9 \\
21.6 \\
21.4 \\
21.4\end{array}$ \\
\hline $\begin{array}{l}11 \\
12 \\
13 \\
14 \\
15\end{array}$ & $\begin{array}{l}30.2 \\
29.0 \\
29.6 \\
30.3 \\
31.1\end{array}$ & $\begin{array}{l}28.7 \\
28.4 \\
28.7 \\
29.2 \\
29.5\end{array}$ & $\begin{array}{l}29.1 \\
28.7 \\
28.9 \\
29.6 \\
29.9\end{array}$ & $\begin{array}{l}27.1 \\
26.8 \\
26.5 \\
26.2 \\
26.7\end{array}$ & $\begin{array}{l}26.4 \\
26.3 \\
26.0 \\
25.8 \\
25.7\end{array}$ & $\begin{array}{l}26.7 \\
26.5 \\
26.1 \\
25.9 \\
26.0\end{array}$ & $\begin{array}{l}22.0 \\
22.3 \\
22.4 \\
22.3 \\
21.3\end{array}$ & $\begin{array}{l}21.3 \\
21.4 \\
21.4 \\
21.3 \\
20.5\end{array}$ & $\begin{array}{l}21.5 \\
21.7 \\
21.7 \\
21.6 \\
20.8\end{array}$ \\
\hline $\begin{array}{l}16 \\
17 \\
18 \\
19 \\
20\end{array}$ & $\begin{array}{l}31.1 \\
32.0 \\
31.2 \\
32.4 \\
31.9\end{array}$ & $\begin{array}{l}29.7 \\
30.1 \\
30.4 \\
30.6 \\
30.2\end{array}$ & $\begin{array}{l}30.2 \\
30.5 \\
30.7 \\
31.0 \\
30.8\end{array}$ & $\begin{array}{l}25.7 \\
25.3 \\
24.7 \\
24.9 \\
24.3\end{array}$ & $\begin{array}{l}25.1 \\
24.7 \\
24.3 \\
24.0 \\
24.1\end{array}$ & $\begin{array}{l}25.4 \\
25.0 \\
24.5 \\
24.3 \\
24.2\end{array}$ & $\begin{array}{l}20.7 \\
20.3 \\
19.8 \\
20.6 \\
20.1\end{array}$ & $\begin{array}{l}20.0 \\
19.5 \\
19.3 \\
19.5 \\
19.1\end{array}$ & $\begin{array}{l}20.3 \\
19.8 \\
19.4 \\
19.8 \\
19.5\end{array}$ \\
\hline $\begin{array}{l}21 \\
22 \\
23 \\
24 \\
25\end{array}$ & $\begin{array}{l}31.6 \\
31.2 \\
30.9 \\
30.7 \\
30.5\end{array}$ & $\begin{array}{l}30.4 \\
30.5 \\
30.2 \\
29.8 \\
29.8\end{array}$ & $\begin{array}{l}31.0 \\
30.8 \\
30.4 \\
30.1 \\
30.0\end{array}$ & $\begin{array}{l}24.6 \\
24.3 \\
23.6 \\
23.1 \\
23.2\end{array}$ & $\begin{array}{l}24.1 \\
23.5 \\
23.1 \\
22.6 \\
22.5\end{array}$ & $\begin{array}{l}24.3 \\
24.0 \\
23.3 \\
22.8 \\
22.8\end{array}$ & $\begin{array}{l}19.2 \\
18.7 \\
18.8 \\
18.0 \\
17.5\end{array}$ & $\begin{array}{l}18.6 \\
18.5 \\
18.0 \\
17.5 \\
17.0\end{array}$ & $\begin{array}{l}18.9 \\
18.6 \\
18.3 \\
17.7 \\
17.3\end{array}$ \\
\hline $\begin{array}{l}26 \\
27 \\
28 \\
29 \\
30 \\
31\end{array}$ & $\begin{array}{l}30.7 \\
30.7 \\
30.6 \\
30.6 \\
30.2 \\
30.0\end{array}$ & $\begin{array}{l}29.6 \\
29.6 \\
29.5 \\
29.6 \\
29.6 \\
29.6\end{array}$ & $\begin{array}{l}29.9 \\
30.0 \\
29.9 \\
29.9 \\
29.8 \\
29.7\end{array}$ & $\begin{array}{r}22.6 \\
22.2 \\
22.3 \\
22.8 \\
23.1 \\
\ldots\end{array}$ & $\begin{array}{r}22.2 \\
22.1 \\
21.9 \\
22.0 \\
21.9 \\
\ldots .\end{array}$ & $\begin{array}{r}22.3 \\
22.1 \\
22.1 \\
22.2 \\
22.1 \\
\ldots\end{array}$ & $\begin{array}{l}17.0 \\
17.2 \\
17.1 \\
16.7 \\
16.2 \\
16.2\end{array}$ & $\begin{array}{l}16.8 \\
16.8 \\
16.7 \\
16.2 \\
16.0 \\
15.8\end{array}$ & $\begin{array}{l}16.9 \\
16.9 \\
16.9 \\
16.4 \\
16.1 \\
16.1\end{array}$ \\
\hline Month & 32.4 & 27.3 & 29.9 & 29.9 & 21.9 & 25.5 & 22.7 & 15.8 & 19.8 \\
\hline
\end{tabular}


Table 34. Daily maximum, minimum, and mean dissolved oxygen concentrations at station 392145081185601, from the Willow Island Dam (upstream) continuousrecording water-quality monitor, June to october 1995.

[..., value not determined]

\begin{tabular}{|c|c|c|c|c|c|c|c|c|c|}
\hline \multirow{3}{*}{ Day } & \multirow{2}{*}{\multicolumn{3}{|c|}{ Dissolved ox }} & \multicolumn{6}{|c|}{ concentration, in milligrams per liter } \\
\hline & & & & & June & & & July & \\
\hline & Maximum & Minimum & Mean & Maximum & Minimum & Mean & Maximum & Minimum & Mean \\
\hline $\begin{array}{l}1 \\
2 \\
3 \\
4 \\
5\end{array}$ & $\begin{array}{l}\cdots \\
\cdots \\
\cdots \\
\cdots\end{array}$ & $\begin{array}{l}\cdots \\
\cdots \\
\cdots \\
\cdots \\
\cdots\end{array}$ & $\begin{array}{l}\cdots \\
\cdots \\
\cdots \\
\cdots \\
\cdots\end{array}$ & $\begin{array}{l}9.7 \\
9.5 \\
9.4 \\
9.6 \\
9.9\end{array}$ & $\begin{array}{l}9.2 \\
9.1 \\
9.1 \\
9.2 \\
9.3\end{array}$ & $\begin{array}{l}9.3 \\
9.2 \\
9.2 \\
9.4 \\
9.6\end{array}$ & $\begin{array}{l}5.7 \\
6.1 \\
6.3 \\
5.9 \\
6.1\end{array}$ & $\begin{array}{l}5.3 \\
5.4 \\
5.5 \\
5.5 \\
5.4\end{array}$ & $\begin{array}{l}5.5 \\
5.6 \\
5.8 \\
5.7 \\
5.6\end{array}$ \\
\hline $\begin{array}{r}6 \\
7 \\
8 \\
9 \\
10\end{array}$ & $\begin{array}{l}\cdots \\
\cdots \\
\cdots \\
\cdots \\
\cdots\end{array}$ & $\begin{array}{l}\cdots \\
\cdots \\
\cdots \\
\cdots \\
\cdots\end{array}$ & $\begin{array}{c}\cdots \\
\cdots \\
\cdots \\
- \\
- \\
-\end{array}$ & $\begin{array}{r}9.5 \\
9.9 \\
11.5 \\
11.2 \\
10.4\end{array}$ & $\begin{array}{l}8.8 \\
8.8 \\
9.6 \\
9.9 \\
9.6\end{array}$ & $\begin{array}{r}9.2 \\
9.4 \\
10.2 \\
10.4 \\
10.1\end{array}$ & $\begin{array}{l}5.9 \\
6.2 \\
7.0 \\
7.5 \\
7.8\end{array}$ & $\begin{array}{l}5.4 \\
5.7 \\
5.8 \\
6.1 \\
6.2\end{array}$ & $\begin{array}{l}5.6 \\
5.9 \\
6.1 \\
6.4 \\
6.9\end{array}$ \\
\hline $\begin{array}{l}11 \\
12 \\
13 \\
14 \\
15\end{array}$ & $\begin{array}{l}\cdots \\
\cdots \\
\cdots \\
\cdots \\
\cdots\end{array}$ & $\begin{array}{l}\cdots \\
\cdots \\
\cdots \\
\cdots \\
\cdots\end{array}$ & $\begin{array}{l}\cdots \\
\cdots \\
\cdots \\
\cdots\end{array}$ & $\begin{array}{r}10.2 \\
9.2 \\
8.1 \\
8.6 \\
9.0\end{array}$ & $\begin{array}{l}9.2 \\
8.1 \\
7.9 \\
7.9 \\
7.9\end{array}$ & $\begin{array}{l}9.7 \\
8.4 \\
8.0 \\
8.1 \\
8.3\end{array}$ & $\begin{array}{l}8.2 \\
8.7 \\
8.2 \\
8.5 \\
8.1\end{array}$ & $\begin{array}{l}6.5 \\
6.6 \\
6.7 \\
7.1 \\
7.0\end{array}$ & $\begin{array}{l}6.9 \\
6.9 \\
7.4 \\
7.7 \\
7.2\end{array}$ \\
\hline $\begin{array}{l}16 \\
17 \\
18 \\
19 \\
20\end{array}$ & $\begin{array}{l}\cdots \\
\cdots \\
\cdots \\
\cdots \\
\cdots\end{array}$ & $\begin{array}{l}\cdots \\
\cdots \\
\cdots \\
\cdots \\
\cdots\end{array}$ & $\begin{array}{l}\cdots \\
\cdots \\
\cdots \\
\cdots\end{array}$ & $\begin{array}{l}8.6 \\
8.9 \\
8.8 \\
9.1 \\
8.7\end{array}$ & $\begin{array}{l}7.9 \\
7.7 \\
8.1 \\
8.0 \\
7.9\end{array}$ & $\begin{array}{l}8.1 \\
8.2 \\
8.3 \\
8.3 \\
8.2\end{array}$ & $\begin{array}{l}8.2 \\
7.9 \\
7.4 \\
7.0 \\
6.9\end{array}$ & $\begin{array}{l}6.8 \\
6.7 \\
6.4 \\
6.0 \\
6.0\end{array}$ & $\begin{array}{l}7.2 \\
7.2 \\
6.8 \\
6.4 \\
6.3\end{array}$ \\
\hline $\begin{array}{l}21 \\
22 \\
23 \\
24 \\
25\end{array}$ & $\begin{array}{l}-. \\
-- \\
-- \\
9.4 \\
9.4\end{array}$ & $\begin{array}{l}\ldots- \\
\cdots \\
\cdots \\
9.2 \\
9.3\end{array}$ & $\begin{array}{l}\ldots \\
\ldots \\
\cdots \\
9.3 \\
9.3\end{array}$ & $\begin{array}{l}8.6 \\
8.2 \\
7.3 \\
7.3 \\
7.0\end{array}$ & $\begin{array}{l}7.8 \\
7.3 \\
6.7 \\
6.6 \\
6.2\end{array}$ & $\begin{array}{l}8.1 \\
7.6 \\
7.1 \\
6.7 \\
6.6\end{array}$ & $\begin{array}{l}7.4 \\
6.2 \\
6.1 \\
6.3 \\
6.5\end{array}$ & $\begin{array}{l}6.0 \\
5.5 \\
5.5 \\
5.4 \\
5.4\end{array}$ & $\begin{array}{l}6.3 \\
5.8 \\
5.8 \\
5.7 \\
5.7\end{array}$ \\
\hline $\begin{array}{l}26 \\
27 \\
28 \\
29 \\
30 \\
31\end{array}$ & $\begin{array}{l}9.3 \\
9.1 \\
9.3 \\
9.4 \\
9.4 \\
9.7\end{array}$ & $\begin{array}{l}9.1 \\
9.0 \\
9.0 \\
9.1 \\
9.2 \\
9.2\end{array}$ & $\begin{array}{l}9.2 \\
9.1 \\
9.2 \\
9.2 \\
9.3 \\
9.4\end{array}$ & $\begin{array}{l}7.4 \\
7.1 \\
7.9 \\
6.8 \\
6.0 \\
\ldots .-\end{array}$ & $\begin{array}{l}6.4 \\
6.3 \\
6.4 \\
6.0 \\
5.5 \\
\ldots .\end{array}$ & $\begin{array}{l}6.7 \\
6.5 \\
6.9 \\
6.5 \\
5.7 \\
\ldots .\end{array}$ & $\begin{array}{l}7.2 \\
8.1 \\
7.9 \\
7.2 \\
8.4 \\
7.9\end{array}$ & $\begin{array}{l}5.8 \\
6.6 \\
6.4 \\
6.6 \\
6.7 \\
6.9\end{array}$ & $\begin{array}{l}6.2 \\
7.1 \\
6.9 \\
6.9 \\
7.2 \\
7.2\end{array}$ \\
\hline Month & $\cdots$ & $-\cdots$ & - . & 11.5 & 5.5 & 8.3 & 8.7 & 5.3 & 6.4 \\
\hline
\end{tabular}


Table 34. Daily maximum, minimum, and mean dissolved oxygen concentrations at station 392145081185601, from the Willow Island Dam (upstream) continuousrecording water-quality monitor, June to october 1995 - Continued.

[..., value not determined]

\begin{tabular}{|c|c|c|c|c|c|c|c|c|c|}
\hline \multirow{3}{*}{ Day } & \multirow{2}{*}{\multicolumn{3}{|c|}{ Dissolved oxy }} & \multicolumn{6}{|c|}{ concentration, in milligrams per liter } \\
\hline & & & & \multirow[b]{2}{*}{ Maximum } & \multicolumn{2}{|l|}{ eptember } & \multicolumn{3}{|c|}{ October } \\
\hline & Maximum & Minimum & Mean & & Minimum & Mean & Maximum & Minimum & Mean \\
\hline $\begin{array}{l}1 \\
2 \\
3 \\
4 \\
5\end{array}$ & $\begin{array}{l}8.4 \\
7.5 \\
7.8 \\
7.4 \\
7.2\end{array}$ & $\begin{array}{l}6.6 \\
6.8 \\
6.9 \\
6.7 \\
6.7\end{array}$ & $\begin{array}{l}7.3 \\
7.2 \\
7.3 \\
7.0 \\
7.0\end{array}$ & $\begin{array}{l}6.6 \\
7.3 \\
6.5 \\
6.6 \\
7.4\end{array}$ & $\begin{array}{l}5.7 \\
6.0 \\
5.9 \\
6.1 \\
6.0\end{array}$ & $\begin{array}{l}6.1 \\
6.3 \\
6.1 \\
6.3 \\
6.6\end{array}$ & $\begin{array}{l}7.5 \\
7.2 \\
7.8 \\
6.9 \\
6.8\end{array}$ & $\begin{array}{l}6.8 \\
6.8 \\
6.9 \\
6.7 \\
6.6\end{array}$ & $\begin{array}{l}7.1 \\
7.0 \\
7.1 \\
6.8 \\
6.7\end{array}$ \\
\hline $\begin{array}{r}6 \\
7 \\
8 \\
9 \\
10\end{array}$ & $\begin{array}{l}7.2 \\
7.0 \\
7.1 \\
7.2 \\
6.9\end{array}$ & $\begin{array}{l}6.8 \\
6.1 \\
6.3 \\
6.7 \\
6.2\end{array}$ & $\begin{array}{l}6.9 \\
6.4 \\
6.8 \\
6.9 \\
6.5\end{array}$ & $\begin{array}{l}7.3 \\
7.0 \\
6.6 \\
6.7 \\
6.7\end{array}$ & $\begin{array}{l}6.2 \\
6.1 \\
6.2 \\
5.9 \\
6.1\end{array}$ & $\begin{array}{l}6.7 \\
6.4 \\
6.3 \\
6.3 \\
6.3\end{array}$ & $\begin{array}{l}6.9 \\
6.8 \\
6.9 \\
6.9 \\
7.2\end{array}$ & $\begin{array}{l}6.6 \\
6.6 \\
6.6 \\
6.6 \\
6.8\end{array}$ & $\begin{array}{l}6.7 \\
6.7 \\
6.8 \\
6.8 \\
6.9\end{array}$ \\
\hline $\begin{array}{l}11 \\
12 \\
13 \\
14 \\
15\end{array}$ & $\begin{array}{l}6.8 \\
6.7 \\
8.3 \\
8.4 \\
8.5\end{array}$ & $\begin{array}{l}6.1 \\
6.1 \\
6.4 \\
6.8 \\
6.7\end{array}$ & $\begin{array}{l}6.3 \\
6.4 \\
6.8 \\
7.4 \\
7.2\end{array}$ & $\begin{array}{l}7.0 \\
6.7 \\
6.3 \\
6.4 \\
6.9\end{array}$ & $\begin{array}{l}6.2 \\
6.3 \\
6.1 \\
6.1 \\
6.1\end{array}$ & $\begin{array}{l}6.4 \\
6.4 \\
6.2 \\
6.2 \\
6.4\end{array}$ & $\begin{array}{l}7.2 \\
7.6 \\
7.6 \\
7.4 \\
7.2\end{array}$ & $\begin{array}{l}6.8 \\
6.8 \\
6.8 \\
6.8 \\
6.8\end{array}$ & $\begin{array}{l}7.0 \\
7.1 \\
7.1 \\
6.9 \\
7.0\end{array}$ \\
\hline $\begin{array}{l}16 \\
17 \\
18 \\
19 \\
20\end{array}$ & $\begin{array}{l}8.6 \\
9.0 \\
7.7 \\
9.6 \\
8.8\end{array}$ & $\begin{array}{l}6.9 \\
6.7 \\
6.6 \\
6.9 \\
6.6\end{array}$ & $\begin{array}{l}7.5 \\
7.4 \\
7.1 \\
7.5 \\
7.4\end{array}$ & $\begin{array}{l}6.5 \\
6.4 \\
6.2 \\
\cdots . \\
6.9\end{array}$ & $\begin{array}{l}6.1 \\
6.0 \\
6.0 \\
\cdots . \\
6.0\end{array}$ & $\begin{array}{l}6.3 \\
6.1 \\
6.1 \\
-.- \\
6.3\end{array}$ & $\begin{array}{l}7.3 \\
7.7 \\
7.3 \\
7.5 \\
7.3\end{array}$ & $\begin{array}{l}7.0 \\
7.1 \\
7.1 \\
7.1 \\
7.0\end{array}$ & $\begin{array}{l}7.1 \\
7.3 \\
7.2 \\
7.3 \\
7.1\end{array}$ \\
\hline $\begin{array}{l}21 \\
22 \\
23 \\
24 \\
25\end{array}$ & $\begin{array}{l}8.6 \\
7.7 \\
7.1 \\
7.3 \\
6.8\end{array}$ & $\begin{array}{l}6.8 \\
6.6 \\
6.4 \\
6.1 \\
5.9\end{array}$ & $\begin{array}{l}7.4 \\
7.0 \\
6.8 \\
6.4 \\
6.3\end{array}$ & $\begin{array}{l}6.3 \\
6.2 \\
6.6 \\
6.7 \\
7.2\end{array}$ & $\begin{array}{l}5.7 \\
5.8 \\
6.1 \\
6.4 \\
6.5\end{array}$ & $\begin{array}{l}6.0 \\
6.0 \\
6.3 \\
6.5 \\
6.7\end{array}$ & $\begin{array}{l}7.2 \\
7.3 \\
7.5 \\
7.6 \\
7.6\end{array}$ & $\begin{array}{l}7.1 \\
7.1 \\
7.2 \\
7.4 \\
7.6\end{array}$ & $\begin{array}{l}7.1 \\
7.2 \\
7.3 \\
7.5 \\
7.6\end{array}$ \\
\hline $\begin{array}{l}26 \\
27 \\
28 \\
29 \\
30 \\
31\end{array}$ & $\begin{array}{l}7.0 \\
6.6 \\
6.8 \\
6.3 \\
6.6 \\
6.3\end{array}$ & $\begin{array}{l}6.1 \\
5.9 \\
5.8 \\
5.8 \\
5.7 \\
5.8\end{array}$ & $\begin{array}{l}6.4 \\
6.2 \\
6.2 \\
6.0 \\
6.0 \\
6.0\end{array}$ & $\begin{array}{l}6.7 \\
6.6 \\
7.5 \\
7.5 \\
7.9 \\
\cdots .\end{array}$ & $\begin{array}{l}6.1 \\
6.1 \\
6.4 \\
6.4 \\
6.6 \\
\cdots .\end{array}$ & $\begin{array}{l}6.4 \\
6.3 \\
6.7 \\
6.7 \\
6.9 \\
-.-\end{array}$ & $\begin{array}{l}7.7 \\
7.7 \\
7.8 \\
7.9 \\
7.9 \\
7.9\end{array}$ & $\begin{array}{l}7.6 \\
7.6 \\
7.6 \\
7.8 \\
7.8 \\
7.9\end{array}$ & $\begin{array}{l}7.6 \\
7.6 \\
7.7 \\
7.9 \\
7.9 \\
7.9\end{array}$ \\
\hline Month & 9.6 & 5.7 & 6.8 & $\cdots$ & $\cdots$ & $\cdots$ & 7.9 & 6.6 & 7.2 \\
\hline
\end{tabular}


Table 35. Daily maximum, minimum, and mean specific conductance at station 392125081193601, from the Willow Island Dam (downstream) continuous-recording water-quality monitor, June to October 1995.

$[\cdots$, value not determined]

\begin{tabular}{|c|c|c|c|c|c|c|c|c|c|}
\hline \multirow{3}{*}{ Day } & \multicolumn{3}{|c|}{ Specific conductance, } & \multirow{2}{*}{\multicolumn{3}{|c|}{$\begin{array}{l}\text { Ins per } \\
\text { June }\end{array}$}} & \multicolumn{3}{|c|}{ at 25 degrees Celsius } \\
\hline & \multicolumn{3}{|c|}{ May } & & & & \multicolumn{3}{|c|}{ July } \\
\hline & Maximum & Minimum & Mean & Maximum & Minimum & Mean & Maximum & Minimum & Mean \\
\hline $\begin{array}{l}1 \\
2 \\
3 \\
4 \\
5\end{array}$ & $\begin{array}{l}\ldots \\
\ldots \\
\ldots \\
\ldots \\
\ldots\end{array}$ & $\begin{array}{l}\ldots \\
\cdots \\
\cdots \\
\cdots \\
\ldots\end{array}$ & $\begin{array}{l}- \\
- \\
- \\
- \\
- \\
-\end{array}$ & $\begin{array}{l}307 \\
309 \\
305 \\
300 \\
309\end{array}$ & $\begin{array}{l}302 \\
305 \\
299 \\
283 \\
292\end{array}$ & $\begin{array}{l}305 \\
307 \\
302 \\
290 \\
301\end{array}$ & $\begin{array}{l}357 \\
388 \\
407 \\
412 \\
413\end{array}$ & $\begin{array}{l}333 \\
357 \\
388 \\
407 \\
407\end{array}$ & $\begin{array}{l}340 \\
375 \\
396 \\
410 \\
411\end{array}$ \\
\hline $\begin{array}{r}6 \\
7 \\
8 \\
9 \\
10\end{array}$ & $\begin{array}{l}\ldots \\
\cdots \\
\cdots \\
\cdots \\
\cdots\end{array}$ & $\begin{array}{l}\cdots \\
\cdots \\
\cdots \\
\cdots \\
\cdots\end{array}$ & $\begin{array}{l}\cdots \\
\cdots \\
\cdots \\
\cdots \\
\cdots\end{array}$ & $\begin{array}{l}322 \\
317 \\
321 \\
322 \\
325\end{array}$ & $\begin{array}{l}309 \\
313 \\
315 \\
320 \\
322\end{array}$ & $\begin{array}{l}317 \\
314 \\
319 \\
320 \\
324\end{array}$ & $\begin{array}{l}411 \\
428 \\
430 \\
435 \\
435\end{array}$ & $\begin{array}{l}407 \\
411 \\
426 \\
430 \\
428\end{array}$ & $\begin{array}{l}408 \\
417 \\
429 \\
432 \\
431\end{array}$ \\
\hline $\begin{array}{l}11 \\
12 \\
13 \\
14 \\
15\end{array}$ & $\begin{array}{l}\cdots \\
\cdots \\
\cdots \\
\cdots \\
\cdots\end{array}$ & $\begin{array}{l}\cdots \\
\cdots \\
\cdots \\
\cdots \\
\cdots\end{array}$ & $\begin{array}{c}\cdots \\
\cdots \\
\cdots \\
\cdots \\
-\end{array}$ & $\begin{array}{l}336 \\
338 \\
321 \\
325 \\
333\end{array}$ & $\begin{array}{l}325 \\
315 \\
314 \\
316 \\
323\end{array}$ & $\begin{array}{l}329 \\
327 \\
318 \\
322 \\
326\end{array}$ & $\begin{array}{l}428 \\
425 \\
434 \\
440 \\
437\end{array}$ & $\begin{array}{l}420 \\
420 \\
422 \\
434 \\
436\end{array}$ & $\begin{array}{l}424 \\
422 \\
426 \\
438 \\
436\end{array}$ \\
\hline $\begin{array}{l}16 \\
17 \\
18 \\
19 \\
20\end{array}$ & $\begin{array}{l}\cdots \\
\cdots \\
\cdots \\
\cdots \\
\cdots\end{array}$ & $\begin{array}{l}\ldots \\
\ldots \\
\cdots \\
\cdots \\
\ldots-\end{array}$ & $\begin{array}{l}\cdots \\
\cdots \\
\cdots \\
\cdots\end{array}$ & $\begin{array}{l}342 \\
345 \\
350 \\
352 \\
356\end{array}$ & $\begin{array}{l}333 \\
341 \\
345 \\
349 \\
351\end{array}$ & $\begin{array}{l}337 \\
342 \\
347 \\
351 \\
354\end{array}$ & $\begin{array}{l}453 \\
470 \\
486 \\
497 \\
478\end{array}$ & $\begin{array}{l}435 \\
450 \\
470 \\
478 \\
454\end{array}$ & $\begin{array}{l}444 \\
455 \\
481 \\
490 \\
466\end{array}$ \\
\hline $\begin{array}{l}21 \\
22 \\
23 \\
24 \\
25\end{array}$ & $\begin{array}{l}\ldots \\
\cdots \\
\cdots \\
\cdots \\
\cdots\end{array}$ & $\begin{array}{l}\cdots \\
\cdots \\
\cdots \\
\cdots \\
\cdots\end{array}$ & $\begin{array}{l}\cdots \\
\cdots \\
\cdots \\
\cdots \\
\cdots\end{array}$ & $\begin{array}{l}357 \\
352 \\
344 \\
346 \\
347\end{array}$ & $\begin{array}{l}351 \\
339 \\
335 \\
343 \\
344\end{array}$ & $\begin{array}{l}354 \\
346 \\
339 \\
345 \\
346\end{array}$ & $\begin{array}{l}455 \\
455 \\
458 \\
469 \\
474\end{array}$ & $\begin{array}{l}452 \\
452 \\
453 \\
458 \\
469\end{array}$ & $\begin{array}{l}453 \\
453 \\
454 \\
463 \\
471\end{array}$ \\
\hline $\begin{array}{l}26 \\
27 \\
28 \\
29 \\
30 \\
31\end{array}$ & $\begin{array}{l}\ldots \\
267 \\
279 \\
279 \\
286 \\
302\end{array}$ & $\begin{array}{l}\ldots \\
257 \\
267 \\
277 \\
277 \\
285\end{array}$ & $\begin{array}{l}\ldots \\
259 \\
275 \\
278 \\
281 \\
293\end{array}$ & $\begin{array}{l}366 \\
367 \\
386 \\
372 \\
354 \\
\ldots\end{array}$ & $\begin{array}{l}345 \\
360 \\
364 \\
348 \\
339 \\
\ldots .\end{array}$ & $\begin{array}{l}352 \\
363 \\
378 \\
353 \\
344 \\
\ldots\end{array}$ & $\begin{array}{l}481 \\
481 \\
503 \\
519 \\
518 \\
512\end{array}$ & $\begin{array}{l}472 \\
474 \\
481 \\
499 \\
510 \\
509\end{array}$ & $\begin{array}{l}476 \\
477 \\
491 \\
511 \\
513 \\
510\end{array}$ \\
\hline Month & $\ldots$ & $\ldots$ & $\cdots$ & 386 & 283 & 332 & 519 & 333 & 445 \\
\hline
\end{tabular}


Table 35. Daily maximum, minimum, and mean specific conductance at station 392125081193601, from the Willow Island Dam (downstream) continuous-recording water-quality monitor, June to october 1995-Continued.

$[\cdots$, value not determined]

\begin{tabular}{|c|c|c|c|c|c|c|c|c|c|}
\hline \multirow{3}{*}{ Day } & \multicolumn{9}{|c|}{ Specific conductance, in microsiemens per centimeter at 25 degrees celsius } \\
\hline & \multicolumn{3}{|c|}{ August } & \multicolumn{3}{|c|}{ September } & \multicolumn{3}{|c|}{ October } \\
\hline & Maximum & Minimum & Mean & Maximum & Minimum & Mean & Maximum & Minimum & Mean \\
\hline $\begin{array}{l}1 \\
2 \\
3 \\
4 \\
5\end{array}$ & $\begin{array}{l}509 \\
521 \\
529 \\
532 \\
533\end{array}$ & $\begin{array}{l}502 \\
504 \\
518 \\
528 \\
531\end{array}$ & $\begin{array}{l}505 \\
512 \\
525 \\
531 \\
532\end{array}$ & $\begin{array}{l}531 \\
531 \\
544 \\
562 \\
574\end{array}$ & $\begin{array}{l}528 \\
529 \\
530 \\
544 \\
562\end{array}$ & $\begin{array}{l}530 \\
530 \\
536 \\
553 \\
569\end{array}$ & $\begin{array}{l}607 \\
600 \\
602 \\
597 \\
572\end{array}$ & $\begin{array}{l}599 \\
592 \\
596 \\
549 \\
548\end{array}$ & $\begin{array}{l}603 \\
596 \\
599 \\
573 \\
556\end{array}$ \\
\hline $\begin{array}{r}6 \\
7 \\
8 \\
9 \\
10\end{array}$ & $\begin{array}{l}541 \\
541 \\
487 \\
481 \\
483\end{array}$ & $\begin{array}{l}531 \\
382 \\
442 \\
440 \\
475\end{array}$ & $\begin{array}{l}536 \\
453 \\
471 \\
461 \\
480\end{array}$ & $\begin{array}{l}588 \\
596 \\
607 \\
626 \\
649\end{array}$ & $\begin{array}{l}574 \\
588 \\
595 \\
607 \\
625\end{array}$ & $\begin{array}{l}581 \\
592 \\
600 \\
616 \\
638\end{array}$ & $\begin{array}{l}587 \\
595 \\
612 \\
619 \\
603\end{array}$ & $\begin{array}{l}572 \\
587 \\
594 \\
600 \\
586\end{array}$ & $\begin{array}{l}581 \\
592 \\
602 \\
612 \\
591\end{array}$ \\
\hline $\begin{array}{l}11 \\
12 \\
13 \\
14 \\
15\end{array}$ & $\begin{array}{l}477 \\
495 \\
496 \\
492 \\
504\end{array}$ & $\begin{array}{l}461 \\
462 \\
490 \\
486 \\
487\end{array}$ & $\begin{array}{l}469 \\
479 \\
493 \\
490 \\
495\end{array}$ & $\begin{array}{l}656 \\
662 \\
667 \\
649 \\
660\end{array}$ & $\begin{array}{l}647 \\
655 \\
649 \\
641 \\
645\end{array}$ & $\begin{array}{l}652 \\
658 \\
661 \\
644 \\
653\end{array}$ & $\begin{array}{l}589 \\
576 \\
569 \\
562 \\
564\end{array}$ & $\begin{array}{l}576 \\
564 \\
561 \\
558 \\
555\end{array}$ & $\begin{array}{l}586 \\
570 \\
563 \\
560 \\
560\end{array}$ \\
\hline $\begin{array}{l}16 \\
17 \\
18 \\
19 \\
20\end{array}$ & $\begin{array}{l}525 \\
525 \\
525 \\
521 \\
522\end{array}$ & $\begin{array}{l}495 \\
522 \\
520 \\
497 \\
497\end{array}$ & $\begin{array}{l}517 \\
524 \\
522 \\
515 \\
504\end{array}$ & $\begin{array}{l}673 \\
675 \\
667 \\
657 \\
659\end{array}$ & $\begin{array}{l}660 \\
666 \\
656 \\
651 \\
651\end{array}$ & $\begin{array}{l}667 \\
672 \\
661 \\
654 \\
655\end{array}$ & $\begin{array}{l}560 \\
548 \\
546 \\
574 \\
573\end{array}$ & $\begin{array}{l}546 \\
525 \\
525 \\
546 \\
563\end{array}$ & $\begin{array}{l}553 \\
537 \\
533 \\
564 \\
569\end{array}$ \\
\hline $\begin{array}{l}21 \\
22 \\
23 \\
24 \\
25\end{array}$ & $\begin{array}{l}536 \\
545 \\
546 \\
532 \\
533\end{array}$ & $\begin{array}{l}512 \\
524 \\
532 \\
528 \\
529\end{array}$ & $\begin{array}{l}525 \\
540 \\
541 \\
530 \\
532\end{array}$ & $\begin{array}{l}\ldots \\
647 \\
636 \\
632 \\
601\end{array}$ & $\begin{array}{l}\cdots \\
621 \\
616 \\
600 \\
579\end{array}$ & $\begin{array}{l}\ldots \\
635 \\
626 \\
614 \\
594\end{array}$ & $\begin{array}{l}572 \\
567 \\
549 \\
561 \\
566\end{array}$ & $\begin{array}{l}563 \\
549 \\
541 \\
543 \\
559\end{array}$ & $\begin{array}{l}567 \\
555 \\
546 \\
554 \\
563\end{array}$ \\
\hline $\begin{array}{l}26 \\
27 \\
28 \\
29 \\
30 \\
31\end{array}$ & $\begin{array}{l}535 \\
535 \\
532 \\
529 \\
531 \\
532\end{array}$ & $\begin{array}{l}533 \\
532 \\
526 \\
526 \\
527 \\
528\end{array}$ & $\begin{array}{l}534 \\
534 \\
528 \\
528 \\
529 \\
531\end{array}$ & $\begin{array}{l}596 \\
587 \\
592 \\
595 \\
606 \\
\ldots\end{array}$ & $\begin{array}{l}579 \\
576 \\
576 \\
590 \\
593 \\
\cdots\end{array}$ & $\begin{array}{l}591 \\
581 \\
584 \\
593 \\
602\end{array}$ & $\begin{array}{l}586 \\
579 \\
590 \\
590 \\
579 \\
584\end{array}$ & $\begin{array}{l}566 \\
562 \\
571 \\
579 \\
573 \\
578\end{array}$ & $\begin{array}{l}576 \\
568 \\
579 \\
586 \\
576 \\
581\end{array}$ \\
\hline Month & 546 & 382 & 512 & ... & $\cdots$ & $\cdots$ & 619 & 525 & 573 \\
\hline
\end{tabular}


Table 36. Daily maximum, minimum, and median $p H$ at station 392125081193601, from the Willow Island Dam (downstream) continuous-recording water-quality monitor, June to october 1995.

$[\cdots$, value not determined]

\begin{tabular}{|c|c|c|c|c|c|c|c|c|c|}
\hline \multirow{3}{*}{ Day } & \multicolumn{9}{|c|}{$\mathrm{pH}$, in standard units } \\
\hline & \multicolumn{3}{|c|}{ May } & \multicolumn{3}{|c|}{ June } & \multicolumn{3}{|c|}{ JuIy } \\
\hline & Maximum & Minimum & Median & Maximum & Minimum & Median & Maximum & Minimum & Median \\
\hline $\begin{array}{l}1 \\
2 \\
3 \\
4 \\
5\end{array}$ & $\begin{array}{l}\cdots \\
\cdots \\
\cdots \\
\cdots\end{array}$ & $\begin{array}{l}\cdots \\
\cdots \\
\cdots \\
\cdots \\
\cdots\end{array}$ & $\begin{array}{l}\cdots \\
\cdots \\
\cdots \\
\cdots\end{array}$ & $\begin{array}{l}7.4 \\
7.4 \\
7.4 \\
7.5 \\
7.6\end{array}$ & $\begin{array}{l}7.4 \\
7.3 \\
7.4 \\
7.4 \\
7.4\end{array}$ & $\begin{array}{l}7.4 \\
7.4 \\
7.4 \\
7.4 \\
7.5\end{array}$ & $\begin{array}{l}7.3 \\
7.3 \\
7.4 \\
7.4 \\
7.4\end{array}$ & $\begin{array}{l}7.2 \\
7.3 \\
7.3 \\
7.4 \\
7.3\end{array}$ & $\begin{array}{l}7.2 \\
7.3 \\
7.3 \\
7.4 \\
7.3\end{array}$ \\
\hline $\begin{array}{r}6 \\
7 \\
8 \\
9 \\
10\end{array}$ & $\begin{array}{l}\cdots \\
\cdots \\
\cdots \\
\cdots \\
\cdots\end{array}$ & $\begin{array}{l}\cdots \\
\cdots \\
\cdots \\
\cdots \\
\cdots\end{array}$ & $\begin{array}{l}\cdots \\
\cdots \\
\cdots \\
\cdots \\
\cdots\end{array}$ & $\begin{array}{l}7.5 \\
7.7 \\
8.0 \\
8.1 \\
7.9\end{array}$ & $\begin{array}{l}7.4 \\
7.4 \\
7.6 \\
7.7 \\
7.7\end{array}$ & $\begin{array}{l}7.5 \\
7.6 \\
7.7 \\
7.9 \\
7.8\end{array}$ & $\begin{array}{l}7.3 \\
7.3 \\
7.3 \\
7.4 \\
7.4\end{array}$ & $\begin{array}{l}7.2 \\
7.2 \\
7.3 \\
7.3 \\
7.3\end{array}$ & $\begin{array}{l}7.2 \\
7.2 \\
7.3 \\
7.3 \\
7.3\end{array}$ \\
\hline $\begin{array}{l}11 \\
12 \\
13 \\
14 \\
15\end{array}$ & $\begin{array}{l}\cdots \\
\cdots \\
\cdots \\
\cdots \\
\cdots\end{array}$ & $\begin{array}{l}\cdots \\
\cdots \\
\cdots \\
\cdots \\
\cdots\end{array}$ & $\begin{array}{l}\cdots \\
\cdots \\
\cdots \\
\cdots\end{array}$ & $\begin{array}{l}7.9 \\
7.7 \\
7.4 \\
7.5 \\
7.6\end{array}$ & $\begin{array}{l}7.7 \\
7.4 \\
7.4 \\
7.4 \\
7.5\end{array}$ & $\begin{array}{l}7.7 \\
7.4 \\
7.4 \\
7.5 \\
7.5\end{array}$ & $\begin{array}{l}7.4 \\
7.4 \\
7.5 \\
7.5 \\
7.4\end{array}$ & $\begin{array}{l}7.3 \\
7.3 \\
7.3 \\
7.4 \\
7.4\end{array}$ & $\begin{array}{l}7.4 \\
7.4 \\
7.4 \\
7.4 \\
7.4\end{array}$ \\
\hline $\begin{array}{l}16 \\
17 \\
18 \\
19 \\
20\end{array}$ & $\begin{array}{l}\cdots \\
\cdots \\
\cdots \\
\cdots\end{array}$ & $\begin{array}{l}\cdots \\
\cdots \\
\cdots \\
\cdots \\
\cdots\end{array}$ & $\begin{array}{l}\cdots \\
\cdots \\
\cdots \\
\cdots\end{array}$ & $\begin{array}{l}7.6 \\
7.6 \\
7.7 \\
7.6 \\
7.6\end{array}$ & $\begin{array}{l}7.5 \\
7.5 \\
7.5 \\
7.5 \\
7.5\end{array}$ & $\begin{array}{l}7.5 \\
7.6 \\
7.6 \\
7.6 \\
7.5\end{array}$ & $\begin{array}{l}7.4 \\
7.4 \\
7.4 \\
7.5 \\
7.4\end{array}$ & $\begin{array}{l}7.3 \\
7.3 \\
7.3 \\
7.3 \\
7.4\end{array}$ & $\begin{array}{l}7.4 \\
7.4 \\
7.4 \\
7.4 \\
7.4\end{array}$ \\
\hline $\begin{array}{l}21 \\
22 \\
23 \\
24 \\
25\end{array}$ & $\begin{array}{l}\cdots \\
\cdots \\
\cdots \\
\cdots\end{array}$ & $\begin{array}{l}\cdots \\
\cdots \\
\cdots \\
\cdots \\
\cdots\end{array}$ & $\begin{array}{l}\cdots \\
\cdots \\
\cdots \\
\cdots\end{array}$ & $\begin{array}{l}7.6 \\
7.5 \\
7.5 \\
7.4 \\
7.4\end{array}$ & $\begin{array}{l}7.5 \\
7.4 \\
7.4 \\
7.4 \\
7.4\end{array}$ & $\begin{array}{l}7.5 \\
7.5 \\
7.4 \\
7.4 \\
7.4\end{array}$ & $\begin{array}{l}7.4 \\
7.4 \\
7.4 \\
7.4 \\
7.4\end{array}$ & $\begin{array}{l}7.4 \\
7.3 \\
7.3 \\
7.3 \\
7.3\end{array}$ & $\begin{array}{l}7.4 \\
7.3 \\
7.3 \\
7.3 \\
7.3\end{array}$ \\
\hline $\begin{array}{l}26 \\
27 \\
28 \\
29 \\
30 \\
31\end{array}$ & $\begin{array}{l}\cdots . \\
7.2 \\
7.3 \\
7.3 \\
7.4 \\
7.4\end{array}$ & $\begin{array}{l}7 . . \\
7.2 \\
7.2 \\
7.2 \\
7.3 \\
7.3\end{array}$ & $\begin{array}{l}\ldots \\
7.2 \\
7.2 \\
7.3 \\
7.3 \\
7.4\end{array}$ & $\begin{array}{l}7.5 \\
7.5 \\
7.6 \\
7.4 \\
7.3\end{array}$ & $\begin{array}{l}7.4 \\
7.4 \\
7.4 \\
7.3 \\
7.2 \\
\cdots\end{array}$ & $\begin{array}{l}7.4 \\
7.4 \\
7.5 \\
7.4 \\
7.2 \\
\cdots\end{array}$ & $\begin{array}{l}7.4 \\
7.4 \\
7.4 \\
7.4 \\
7.4 \\
7.5\end{array}$ & $\begin{array}{l}7.3 \\
7.3 \\
7.3 \\
7.3 \\
7.3 \\
7.3\end{array}$ & $\begin{array}{l}7.4 \\
7.3 \\
7.3 \\
7.3 \\
7.3 \\
7.4\end{array}$ \\
\hline Month & $\cdots$ & $\cdots$ & $\cdots$ & 8.1 & 7.2 & $\cdots$ & 7.5 & 7.2 & $\cdots$ \\
\hline
\end{tabular}


Table 36. Daily maximum, minimum, and median $p H$ at station 392125081193601, from the Willow Island Dam (downstream) continuous-recording water-quality monitor, June to October 1995-. Continued.

[.., value not determined]

\begin{tabular}{|c|c|c|c|c|c|c|c|c|c|}
\hline \multirow{3}{*}{ Day } & \multicolumn{9}{|c|}{$\mathrm{pH}$, in standard units } \\
\hline & \multicolumn{3}{|c|}{ August } & \multicolumn{3}{|c|}{ September } & \multicolumn{3}{|c|}{ October } \\
\hline & Maximum & Minimum & Median & Maximum & Minimum & Median & Maximum & Minimum & Median \\
\hline $\begin{array}{l}1 \\
2 \\
3 \\
4 \\
5\end{array}$ & $\begin{array}{l}7.4 \\
7.4 \\
7.4 \\
7.4 \\
7.4\end{array}$ & $\begin{array}{l}7.4 \\
7.4 \\
7.4 \\
7.3 \\
7.4\end{array}$ & $\begin{array}{l}7.4 \\
7.4 \\
7.4 \\
7.4 \\
7.4\end{array}$ & $\begin{array}{l}7.2 \\
7.2 \\
7.2 \\
7.2 \\
7.2\end{array}$ & $\begin{array}{l}7.1 \\
7.1 \\
7.1 \\
7.1 \\
7.1\end{array}$ & $\begin{array}{l}7.2 \\
7.1 \\
7.1 \\
7.1 \\
7.2\end{array}$ & $\begin{array}{l}7.2 \\
7.2 \\
7.2 \\
7.1 \\
7.2\end{array}$ & $\begin{array}{l}7.2 \\
7.2 \\
7.1 \\
7.1 \\
7.1\end{array}$ & $\begin{array}{l}7.2 \\
7.2 \\
7.1 \\
7.1 \\
7.1\end{array}$ \\
\hline $\begin{array}{r}6 \\
7 \\
8 \\
9 \\
10\end{array}$ & $\begin{array}{l}7.5 \\
7.4 \\
7.5 \\
7.6 \\
7.5\end{array}$ & $\begin{array}{l}7.4 \\
7.2 \\
7.3 \\
7.5 \\
7.4\end{array}$ & $\begin{array}{l}7.5 \\
7.3 \\
7.4 \\
7.5 \\
7.5\end{array}$ & $\begin{array}{l}7.2 \\
7.2 \\
7.2 \\
7.2 \\
7.3\end{array}$ & $\begin{array}{l}7.2 \\
7.1 \\
7.2 \\
7.2 \\
7.2\end{array}$ & $\begin{array}{l}7.2 \\
7.2 \\
7.2 \\
7.2 \\
7.2\end{array}$ & $\begin{array}{l}7.2 \\
7.2 \\
7.2 \\
7.3 \\
7.3\end{array}$ & $\begin{array}{l}7.2 \\
7.2 \\
7.2 \\
7.2 \\
7.3\end{array}$ & $\begin{array}{l}7.2 \\
7.2 \\
7.2 \\
7.2 \\
7.3\end{array}$ \\
\hline $\begin{array}{l}11 \\
12 \\
13 \\
14 \\
15\end{array}$ & $\begin{array}{l}7.4 \\
7.4 \\
7.4 \\
7.4 \\
7.4\end{array}$ & $\begin{array}{l}7.4 \\
7.4 \\
7.3 \\
7.3 \\
7.4\end{array}$ & $\begin{array}{l}7.4 \\
7.4 \\
7.4 \\
7.4 \\
7.4\end{array}$ & $\begin{array}{l}7.3 \\
7.3 \\
7.3 \\
7.3 \\
7.4\end{array}$ & $\begin{array}{l}7.2 \\
7.3 \\
7.2 \\
7.3 \\
7.3\end{array}$ & $\begin{array}{l}7.3 \\
7.3 \\
7.3 \\
7.3 \\
7.3\end{array}$ & $\begin{array}{l}7 \cdot 3 \\
7 \cdot 3 \\
7 \cdot 3 \\
7 \cdot 3 \\
7 \cdot 3\end{array}$ & $\begin{array}{l}7.3 \\
7.3 \\
7 \cdot 3 \\
7.2 \\
7.2\end{array}$ & $\begin{array}{l}7 \cdot 3 \\
7 \cdot 3 \\
7 \cdot 3 \\
7 \cdot 2 \\
7.3\end{array}$ \\
\hline $\begin{array}{l}16 \\
17 \\
18 \\
19 \\
20\end{array}$ & $\begin{array}{l}7.6 \\
7.6 \\
7.5 \\
7.5 \\
7.5\end{array}$ & $\begin{array}{l}7.4 \\
7.4 \\
7.4 \\
7.4 \\
7.2\end{array}$ & $\begin{array}{l}7.5 \\
7.5 \\
7.4 \\
7.4 \\
7.3\end{array}$ & $\begin{array}{l}7.4 \\
7.3 \\
7.4 \\
7.3 \\
7.4\end{array}$ & $\begin{array}{l}7.3 \\
7 \cdot 3 \\
7 \cdot 3 \\
7.3 \\
7.3\end{array}$ & $\begin{array}{l}7.3 \\
7.3 \\
7.3 \\
7.3 \\
7.4\end{array}$ & $\begin{array}{l}7.3 \\
7.3 \\
7.4 \\
7.4 \\
7.4\end{array}$ & $\begin{array}{l}7.3 \\
7.3 \\
7.3 \\
7.4 \\
7.4\end{array}$ & $\begin{array}{l}7.3 \\
7.3 \\
7.3 \\
7.4 \\
7.4\end{array}$ \\
\hline $\begin{array}{l}21 \\
22 \\
23 \\
24 \\
25\end{array}$ & $\begin{array}{l}7.3 \\
7.3 \\
7.3 \\
7.2 \\
7.3\end{array}$ & $\begin{array}{l}7.2 \\
7.2 \\
7.2 \\
7.2 \\
7.2\end{array}$ & $\begin{array}{l}7.3 \\
7.3 \\
7 \cdot 3 \\
7 \cdot 2 \\
7.2\end{array}$ & $\begin{array}{l}7.4 \\
7.4 \\
7.4 \\
7.4\end{array}$ & $\begin{array}{l}7.3 \\
7.3 \\
7.4 \\
7.3\end{array}$ & $\begin{array}{l}7.3 \\
7.3 \\
7.4 \\
7.4\end{array}$ & $\begin{array}{l}7.5 \\
7.5 \\
7.5 \\
7.6 \\
7.6\end{array}$ & $\begin{array}{l}7.4 \\
7.4 \\
7.5 \\
7.5 \\
7.6\end{array}$ & $\begin{array}{l}7.4 \\
7.5 \\
7.5 \\
7.5 \\
7.6\end{array}$ \\
\hline $\begin{array}{l}26 \\
27 \\
28 \\
29 \\
30 \\
31\end{array}$ & $\begin{array}{l}7.3 \\
7.3 \\
7.3 \\
7.3 \\
7.3 \\
7.3\end{array}$ & $\begin{array}{l}7.2 \\
7.2 \\
7.2 \\
7.2 \\
7.2 \\
7.2\end{array}$ & $\begin{array}{l}7.3 \\
7.3 \\
7.3 \\
7.2 \\
7.2 \\
7.2\end{array}$ & $\begin{array}{l}7.3 \\
7.3 \\
7.3 \\
7.3 \\
7.3 \\
\ldots .-\end{array}$ & $\begin{array}{l}7.3 \\
7.3 \\
7.2 \\
7.2 \\
7.2 \\
\cdots-.\end{array}$ & $\begin{array}{l}7.3 \\
7.3 \\
7.3 \\
7.3 \\
7.3 \\
-.-\end{array}$ & $\begin{array}{l}7.6 \\
7.6 \\
7.6 \\
7.6 \\
7.7 \\
7.7\end{array}$ & $\begin{array}{l}7.6 \\
7.6 \\
7.6 \\
7.6 \\
7.6 \\
7.6\end{array}$ & $\begin{array}{l}7.6 \\
7.6 \\
7.6 \\
7.6 \\
7.6 \\
7.7\end{array}$ \\
\hline Month & 7.6 & 7.2 & $\cdots$ & $\cdots$ & $\cdots$ & $\cdots$ & 7.7 & 7.1 & $\cdots$ \\
\hline
\end{tabular}


Table 37. Daily maximum, minimum, and mean water temperature at station 392125081193601, from the Willow Island Dam (downstream) continuous-recording water-quality monitor, June to October 1995.

[.., value not determined]

\begin{tabular}{|c|c|c|c|c|c|c|c|c|c|}
\hline \multirow{3}{*}{ Day } & \multicolumn{9}{|c|}{ Water temperature, in degrees celsius } \\
\hline & \multicolumn{3}{|c|}{ May } & \multicolumn{3}{|c|}{ June } & \multicolumn{3}{|c|}{ July } \\
\hline & Maximum & Minimum & Mean & Maximum & Minimum & Mean & Maximum & Minimum & Mean \\
\hline $\begin{array}{l}1 \\
2 \\
3 \\
4 \\
5\end{array}$ & $\begin{array}{l}\cdots \\
\cdots \\
\cdots \\
\cdots \\
\cdots\end{array}$ & $\begin{array}{l}\cdots \\
\cdots \\
\cdots \\
\cdots \\
\cdots\end{array}$ & $\begin{array}{l}\cdots \\
\cdots \\
\cdots \\
\cdots\end{array}$ & $\begin{array}{l}20.4 \\
20.5 \\
20.4 \\
20.6 \\
21.5\end{array}$ & $\begin{array}{l}20.0 \\
20.2 \\
20.1 \\
20.3 \\
20.6\end{array}$ & $\begin{array}{l}20.2 \\
20.4 \\
20.2 \\
20.4 \\
21.0\end{array}$ & $\begin{array}{l}27.1 \\
26.7 \\
26.8 \\
26.6 \\
26.8\end{array}$ & $\begin{array}{l}26.6 \\
26.3 \\
26.2 \\
26.3 \\
26.4\end{array}$ & $\begin{array}{l}26.9 \\
26.5 \\
26.5 \\
26.5 \\
26.6\end{array}$ \\
\hline $\begin{array}{r}6 \\
7 \\
8 \\
9 \\
10\end{array}$ & $\begin{array}{l}\cdots \\
\cdots \\
\cdots \\
\cdots\end{array}$ & $\begin{array}{l}\cdots \\
\cdots \\
\cdots \\
\cdots \\
\cdots\end{array}$ & $\begin{array}{l}\cdots \\
\cdots \\
\cdots \\
\cdots\end{array}$ & $\begin{array}{l}21.8 \\
22.5 \\
23.0 \\
23.2 \\
23.4\end{array}$ & $\begin{array}{l}21.3 \\
21.7 \\
22.3 \\
22.7 \\
23.0\end{array}$ & $\begin{array}{l}21.5 \\
22.1 \\
22.6 \\
23.0 \\
23.2\end{array}$ & $\begin{array}{l}26.8 \\
26.8 \\
27.0 \\
27.0 \\
27.0\end{array}$ & $\begin{array}{l}26.5 \\
26.6 \\
26.5 \\
26.6 \\
26.7\end{array}$ & $\begin{array}{l}26.6 \\
26.7 \\
26.7 \\
26.7 \\
26.9\end{array}$ \\
\hline $\begin{array}{l}11 \\
12 \\
13 \\
14 \\
15\end{array}$ & $\begin{array}{l}\cdots \\
\cdots \\
\cdots \\
\cdots \\
\cdots\end{array}$ & $\begin{array}{l}\cdots \\
\cdots \\
\cdots \\
\cdots \\
\cdots\end{array}$ & $\begin{array}{l}\cdots \\
\cdots \\
\cdots \\
\cdots\end{array}$ & $\begin{array}{l}23.8 \\
23.7 \\
23.0 \\
23.4 \\
23.6\end{array}$ & $\begin{array}{l}23.2 \\
22.8 \\
22.5 \\
22.6 \\
22.7\end{array}$ & $\begin{array}{l}23.5 \\
23.2 \\
22.8 \\
23.0 \\
23.1\end{array}$ & $\begin{array}{l}27.2 \\
27.7 \\
28.0 \\
28.3 \\
28.5\end{array}$ & $\begin{array}{l}26.9 \\
27.1 \\
27.4 \\
27.6 \\
27.8\end{array}$ & $\begin{array}{l}27.1 \\
27.4 \\
27.6 \\
27.8 \\
28.2\end{array}$ \\
\hline $\begin{array}{l}16 \\
17 \\
18 \\
19 \\
20\end{array}$ & $\begin{array}{l}\cdots \\
\cdots \\
\cdots \\
\cdots \\
\cdots\end{array}$ & $\begin{array}{l}\ldots \\
\cdots \\
\cdots \\
\cdots\end{array}$ & $\begin{array}{l}\ldots \\
\ldots \\
\ldots \\
\cdots\end{array}$ & $\begin{array}{l}23.8 \\
24.1 \\
24.7 \\
24.9 \\
25.0\end{array}$ & $\begin{array}{l}23.2 \\
23.4 \\
24.0 \\
24.2 \\
24.5\end{array}$ & $\begin{array}{l}23.5 \\
23.8 \\
24.3 \\
24.5 \\
24.7\end{array}$ & $\begin{array}{l}28.7 \\
28.9 \\
28.7 \\
28.8 \\
28.9\end{array}$ & $\begin{array}{l}28.1 \\
28.5 \\
28.4 \\
28.1 \\
28.3\end{array}$ & $\begin{array}{l}28.3 \\
28.7 \\
28.6 \\
28.5 \\
28.5\end{array}$ \\
\hline $\begin{array}{l}21 \\
22 \\
23 \\
24 \\
25\end{array}$ & $\begin{array}{l}\cdots \\
\cdots \\
\cdots \\
\cdots\end{array}$ & $\begin{array}{l}\cdots \\
\cdots \\
\cdots \\
\cdots\end{array}$ & $\begin{array}{l}\cdots \\
\cdots \\
\cdots \\
\cdots\end{array}$ & $\begin{array}{l}25.6 \\
25.6 \\
25.7 \\
25.7 \\
26.3\end{array}$ & $\begin{array}{l}24.8 \\
25.2 \\
25.4 \\
25.3 \\
25.7\end{array}$ & $\begin{array}{l}25.1 \\
25.4 \\
25.5 \\
25.5 \\
25.9\end{array}$ & $\begin{array}{l}28.9 \\
28.9 \\
28.8 \\
28.8 \\
28.8\end{array}$ & $\begin{array}{l}28.4 \\
28.3 \\
28.6 \\
28.6 \\
28.6\end{array}$ & $\begin{array}{l}28.6 \\
28.6 \\
28.7 \\
28.7 \\
28.7\end{array}$ \\
\hline $\begin{array}{l}26 \\
27 \\
28 \\
29 \\
30 \\
31\end{array}$ & $\begin{array}{r}\cdots \\
19.8 \\
19.9 \\
19.8 \\
19.7 \\
20.2\end{array}$ & $\begin{array}{c}\cdots \\
19.2 \\
19.4 \\
19.5 \\
19.6 \\
19.5\end{array}$ & $\begin{array}{r}\cdots \\
19.5 \\
19.6 \\
19.7 \\
19.7 \\
19.8\end{array}$ & $\begin{array}{r}26.7 \\
27.2 \\
27.6 \\
27.6 \\
27.4 \\
\ldots\end{array}$ & $\begin{array}{r}26.2 \\
26.5 \\
26.9 \\
27.0 \\
26.9 \\
\ldots\end{array}$ & $\begin{array}{r}26.4 \\
26.8 \\
27.2 \\
27.3 \\
27.1 \\
\ldots\end{array}$ & $\begin{array}{l}29.0 \\
29.1 \\
29.3 \\
29.5 \\
29.9 \\
30.4\end{array}$ & $\begin{array}{l}28.5 \\
28.6 \\
28.8 \\
29.1 \\
29.4 \\
29.6\end{array}$ & $\begin{array}{l}28.7 \\
28.8 \\
29.0 \\
29.3 \\
29.7 \\
29.9\end{array}$ \\
\hline Month & $\cdots$ & $\cdots$ & $\cdots$ & 27.6 & 20.0 & 23.8 & 30.4 & 26.2 & 27.9 \\
\hline
\end{tabular}


Table 37. Daily maximum, minimum, and mean water temperature at station 392125081193601, from the Willow Island Dam (downstream) continuous-recording water-quality monitor, June to October 1995-Continued.

$[\cdots$, value not determined]

\begin{tabular}{|c|c|c|c|c|c|c|c|c|c|}
\hline \multirow{3}{*}{ Day } & \multicolumn{9}{|c|}{ Water temperature, in degrees Celsius } \\
\hline & \multicolumn{3}{|c|}{ August } & \multicolumn{3}{|c|}{ September } & \multicolumn{3}{|c|}{ October } \\
\hline & Maximum & Minimum & Mean & Maximum & Minimum & Mean & Maximum & Minimum & Mean \\
\hline $\begin{array}{l}1 \\
2 \\
3 \\
4 \\
5\end{array}$ & $\begin{array}{l}30.2 \\
30.4 \\
30.3 \\
30.5 \\
30.5\end{array}$ & $\begin{array}{l}29.7 \\
29.7 \\
30.1 \\
30.2 \\
30.1\end{array}$ & $\begin{array}{l}29.9 \\
30.1 \\
30.2 \\
30.3 \\
30.3\end{array}$ & $\begin{array}{l}29.7 \\
29.3 \\
28.7 \\
28.6 \\
28.9\end{array}$ & $\begin{array}{l}29.3 \\
28.7 \\
28.3 \\
28.1 \\
28.2\end{array}$ & $\begin{array}{l}29.6 \\
29.0 \\
28.5 \\
28.4 \\
28.5\end{array}$ & $\begin{array}{l}22.2 \\
22.4 \\
22.2 \\
22.2 \\
22.0\end{array}$ & $\begin{array}{l}22.0 \\
22.1 \\
22.0 \\
22.0 \\
21.9\end{array}$ & $\begin{array}{l}22.1 \\
22.2 \\
22.1 \\
22.1 \\
22.0\end{array}$ \\
\hline $\begin{array}{r}5 \\
7 \\
8 \\
9 \\
10\end{array}$ & $\begin{array}{l}30.1 \\
29.7 \\
29.3 \\
29.4 \\
29.5\end{array}$ & $\begin{array}{l}29.5 \\
27.4 \\
28.6 \\
28.8 \\
29.0\end{array}$ & $\begin{array}{l}29.7 \\
28.5 \\
29.0 \\
29.0 \\
29.3\end{array}$ & $\begin{array}{l}28.7 \\
28.2 \\
28.1 \\
27.9 \\
27.6\end{array}$ & $\begin{array}{l}28.2 \\
27.7 \\
27.6 \\
27.6 \\
26.9\end{array}$ & $\begin{array}{l}28.4 \\
28.0 \\
27.9 \\
27.7 \\
27.3\end{array}$ & $\begin{array}{l}22.0 \\
21.9 \\
21.5 \\
21.5 \\
21.5\end{array}$ & $\begin{array}{l}21.8 \\
21.6 \\
21.4 \\
21.2 \\
21.1\end{array}$ & $\begin{array}{l}21.9 \\
21.8 \\
21.5 \\
21.3 \\
21.3\end{array}$ \\
\hline $\begin{array}{l}11 \\
12 \\
13 \\
14 \\
15\end{array}$ & $\begin{array}{l}29.5 \\
28.9 \\
29.3 \\
29.9 \\
30.1\end{array}$ & $\begin{array}{l}28.8 \\
28.5 \\
28.8 \\
29.2 \\
29.6\end{array}$ & $\begin{array}{l}29.1 \\
28.7 \\
29.0 \\
29.5 \\
29.8\end{array}$ & $\begin{array}{l}26.9 \\
26.7 \\
26.5 \\
26.1 \\
26.2\end{array}$ & $\begin{array}{l}26.5 \\
26.5 \\
26.1 \\
25.9 \\
25.9\end{array}$ & $\begin{array}{l}26.7 \\
26.6 \\
26.3 \\
26.0 \\
26.0\end{array}$ & $\begin{array}{l}21.6 \\
21.7 \\
21.7 \\
21.6 \\
21.2\end{array}$ & $\begin{array}{l}21.2 \\
21.4 \\
21.4 \\
21.2 \\
20.5\end{array}$ & $\begin{array}{l}21.4 \\
21.5 \\
21.6 \\
21.5 \\
20.7\end{array}$ \\
\hline $\begin{array}{l}16 \\
17 \\
18 \\
19 \\
20\end{array}$ & $\begin{array}{l}30.5 \\
30.8 \\
30.9 \\
31.2 \\
31.0\end{array}$ & $\begin{array}{l}29.9 \\
30.3 \\
30.5 \\
30.6 \\
30.6\end{array}$ & $\begin{array}{l}30.2 \\
30.5 \\
30.7 \\
30.9 \\
30.8\end{array}$ & $\begin{array}{l}25.9 \\
25.2 \\
24.8 \\
24.4 \\
24.2\end{array}$ & $\begin{array}{l}25.2 \\
24.8 \\
24.4 \\
24.1 \\
24.1\end{array}$ & $\begin{array}{l}25.5 \\
25.1 \\
24.6 \\
24.2 \\
24.2\end{array}$ & $\begin{array}{l}20.5 \\
20.0 \\
19.6 \\
19.9 \\
19.7\end{array}$ & $\begin{array}{l}19.9 \\
19.3 \\
19.0 \\
19.5 \\
19.2\end{array}$ & $\begin{array}{l}20.1 \\
19.6 \\
19.4 \\
19.7 \\
19.5\end{array}$ \\
\hline $\begin{array}{l}21 \\
22 \\
23 \\
24 \\
25\end{array}$ & $\begin{array}{l}31.0 \\
31.1 \\
30.7 \\
30.2 \\
30.3\end{array}$ & $\begin{array}{l}30.6 \\
30.5 \\
30.1 \\
29.8 \\
29.7\end{array}$ & $\begin{array}{l}30.8 \\
30.8 \\
30.4 \\
30.0 \\
30.0\end{array}$ & $\begin{array}{r}\cdots \\
24.2 \\
23.6 \\
23.0 \\
22.8\end{array}$ & $\begin{array}{r}-\cdots \\
23.6 \\
23.0 \\
22.7 \\
22.6\end{array}$ & $\begin{array}{r}\cdots \\
24.0 \\
23.2 \\
22.8 \\
22.7\end{array}$ & $\begin{array}{l}19.3 \\
18.7 \\
18.7 \\
18.1 \\
17.6\end{array}$ & $\begin{array}{l}18.5 \\
18.4 \\
18.1 \\
17.6 \\
17.1\end{array}$ & $\begin{array}{l}18.9 \\
18.6 \\
18.4 \\
17.9 \\
17.4\end{array}$ \\
\hline $\begin{array}{l}26 \\
27 \\
28 \\
29 \\
30 \\
31\end{array}$ & $\begin{array}{l}30.1 \\
30.2 \\
30.0 \\
29.8 \\
30.1 \\
29.8\end{array}$ & $\begin{array}{l}29.6 \\
29.7 \\
29.6 \\
29.5 \\
29.7 \\
29.5\end{array}$ & $\begin{array}{l}29.8 \\
29.9 \\
29.8 \\
29.7 \\
29.8 \\
29.7\end{array}$ & $\begin{array}{l}22.6 \\
22.2 \\
22.2 \\
22.3 \\
22.2\end{array}$ & $\begin{array}{r}22.2 \\
22.1 \\
21.8 \\
21.8 \\
21.9 \\
\ldots .\end{array}$ & $\begin{array}{c}22.4 \\
22.1 \\
22.1 \\
22.1 \\
22.1 \\
\ldots\end{array}$ & $\begin{array}{l}17.1 \\
17.2 \\
17.2 \\
16.7 \\
16.3 \\
16.2\end{array}$ & $\begin{array}{l}16.9 \\
16.9 \\
16.7 \\
16.3 \\
16.2 \\
16.0\end{array}$ & $\begin{array}{l}17.0 \\
17.0 \\
17.0 \\
16.5 \\
16.2 \\
16.2\end{array}$ \\
\hline Month & 31.2 & 27.4 & 29.9 & $\ldots$ & $\cdots$ & $\cdots$ & 22.4 & 16.0 & 19.8 \\
\hline
\end{tabular}


Table 38. Daily maximum, minimum, and mean dissolved oxygen concentrations at station 392125081193601, from the Willow Island Dam (downstream) continuous recording water-quality monitor, June to october 1995.

$[\cdots$, value not determined $]$

\begin{tabular}{|c|c|c|c|c|c|c|c|c|c|}
\hline \multirow{3}{*}{ Day } & \multicolumn{9}{|c|}{ Dissolved oxygen concentration, in milligrams per liter } \\
\hline & \multicolumn{3}{|c|}{ May } & \multicolumn{3}{|c|}{ June } & \multicolumn{3}{|c|}{ July } \\
\hline & Maximum & Minimum & Mean & Maximum & Minimum & Mean & Maximum & Minimum & Mean \\
\hline $\begin{array}{l}1 \\
2 \\
3 \\
4 \\
5\end{array}$ & $\begin{array}{l}\cdots \\
\cdots \\
\cdots \\
\cdots\end{array}$ & $\begin{array}{l}\cdots \\
\cdots \\
\cdots \\
\cdots\end{array}$ & $\begin{array}{l}\cdots \\
\cdots \\
\cdots \\
\cdots\end{array}$ & $\begin{array}{l}9.5 \\
9.4 \\
9.4 \\
9.6 \\
9.8\end{array}$ & $\begin{array}{l}9.3 \\
9.1 \\
9.2 \\
9.2 \\
9.4\end{array}$ & $\begin{array}{l}9.4 \\
9.3 \\
9.3 \\
9.4 \\
9.6\end{array}$ & $\begin{array}{l}5.9 \\
6.2 \\
6.3 \\
6.3 \\
6.2\end{array}$ & $\begin{array}{l}5.7 \\
5.8 \\
6.0 \\
6.0 \\
5.9\end{array}$ & $\begin{array}{l}5.8 \\
6.0 \\
6.2 \\
6.2 \\
6.0\end{array}$ \\
\hline $\begin{array}{r}6 \\
7 \\
8 \\
9 \\
10\end{array}$ & $\begin{array}{l}\cdots \\
\cdots \\
\cdots \\
\cdots \\
\end{array}$ & $\begin{array}{l}\cdots \\
\cdots \\
\cdots \\
\cdots\end{array}$ & $\begin{array}{l}\cdots \\
\cdots \\
\cdots \\
\cdots \\
\cdots\end{array}$ & $\begin{array}{r}9.6 \\
9.8 \\
10.5 \\
10.7 \\
10.4\end{array}$ & $\begin{array}{r}8.9 \\
8.8 \\
9.6 \\
10.0 \\
9.8\end{array}$ & $\begin{array}{r}9.2 \\
9.3 \\
10.0 \\
10.3 \\
10.1\end{array}$ & $\begin{array}{l}6.3 \\
6.4 \\
6.5 \\
7.1 \\
7.1\end{array}$ & $\begin{array}{l}5.8 \\
6.2 \\
6.2 \\
6.4 \\
6.8\end{array}$ & $\begin{array}{l}6.0 \\
6.3 \\
6.3 \\
6.7 \\
7.0\end{array}$ \\
\hline $\begin{array}{l}11 \\
12 \\
13 \\
14 \\
15\end{array}$ & $\begin{array}{l}\cdots \\
\cdots \\
\cdots \\
\cdots\end{array}$ & $\begin{array}{l}\cdots \\
\cdots \\
\cdots \\
\cdots\end{array}$ & $\begin{array}{l}\cdots \\
\cdots \\
\cdots \\
\cdots \\
\cdots\end{array}$ & $\begin{array}{r}10.0 \\
9.3 \\
8.3 \\
8.4 \\
8.9\end{array}$ & $\begin{array}{l}9.3 \\
8.3 \\
8.1 \\
8.0 \\
8.2\end{array}$ & $\begin{array}{l}9.8 \\
8.6 \\
8.1 \\
8.2 \\
8.5\end{array}$ & $\begin{array}{l}7.4 \\
7.6 \\
8.0 \\
7.9 \\
7.4\end{array}$ & $\begin{array}{l}7.0 \\
7.1 \\
7.2 \\
7.3 \\
7.1\end{array}$ & $\begin{array}{l}7.2 \\
7.3 \\
7.6 \\
7.5 \\
7.3\end{array}$ \\
\hline $\begin{array}{l}16 \\
17 \\
18 \\
19 \\
20\end{array}$ & $\begin{array}{l}\cdots \\
\cdots \\
\cdots \\
\cdots\end{array}$ & $\begin{array}{l}\cdots \\
\cdots \\
\cdots \\
\cdots \\
\cdots\end{array}$ & $\begin{array}{l}\cdots \\
\cdots \\
\cdots \\
\cdots\end{array}$ & $\begin{array}{l}8.7 \\
8.8 \\
8.8 \\
8.6 \\
8.5\end{array}$ & $\begin{array}{l}8.5 \\
8.2 \\
8.4 \\
8.2 \\
8.1\end{array}$ & $\begin{array}{l}8.6 \\
8.5 \\
8.6 \\
8.4 \\
8.3\end{array}$ & $\begin{array}{l}7.4 \\
7.9 \\
7.4 \\
7.2 \\
6.7\end{array}$ & $\begin{array}{l}7.1 \\
7.0 \\
6.9 \\
6.4 \\
6.2\end{array}$ & $\begin{array}{l}7.3 \\
7.4 \\
7.1 \\
6.7 \\
6.4\end{array}$ \\
\hline $\begin{array}{l}21 \\
22 \\
23 \\
24 \\
25\end{array}$ & $\begin{array}{l}\cdots \\
\cdots \\
\cdots \\
\cdots\end{array}$ & $\begin{array}{l}\cdots \\
\cdots \\
\cdots \\
\cdots\end{array}$ & $\begin{array}{l}\cdots \\
\cdots \\
\cdots \\
\cdots\end{array}$ & $\begin{array}{l}8.2 \\
8.0 \\
7.7 \\
7.1 \\
7.0\end{array}$ & $\begin{array}{l}7.9 \\
7.7 \\
7.1 \\
6.9 \\
6.7\end{array}$ & $\begin{array}{l}8.1 \\
7.8 \\
7.4 \\
7.0 \\
6.9\end{array}$ & $\begin{array}{l}6.8 \\
6.1 \\
6.1 \\
6.0 \\
6.1\end{array}$ & $\begin{array}{l}6.1 \\
5.9 \\
5.9 \\
5.6 \\
5.7\end{array}$ & $\begin{array}{l}6.4 \\
6.0 \\
6.0 \\
5.9 \\
5.9\end{array}$ \\
\hline $\begin{array}{l}26 \\
27 \\
28 \\
29 \\
30 \\
31\end{array}$ & $\begin{array}{l}\ldots . . \\
9.2 \\
9.4 \\
9.4 \\
9.8 \\
9.7\end{array}$ & $\begin{array}{l}-. . \\
9.1 \\
9.1 \\
9.2 \\
9.3 \\
9.3\end{array}$ & $\begin{array}{l}\cdots \\
9.2 \\
9.2 \\
9.3 \\
9.4 \\
9.5\end{array}$ & $\begin{array}{l}7.0 \\
6.9 \\
7.5 \\
7.2 \\
6.6 \\
\cdots\end{array}$ & $\begin{array}{l}6.7 \\
6.6 \\
6.6 \\
6.5 \\
5.8 \\
\ldots . .\end{array}$ & $\begin{array}{l}6.8 \\
6.7 \\
7.1 \\
6.8 \\
6.0 \\
\cdots .\end{array}$ & $\begin{array}{l}6.4 \\
6.9 \\
6.9 \\
6.9 \\
7.2 \\
7.4\end{array}$ & $\begin{array}{l}5.9 \\
6.3 \\
6.2 \\
6.6 \\
6.6 \\
6.8\end{array}$ & $\begin{array}{l}6.2 \\
6.5 \\
6.5 \\
6.7 \\
6.9 \\
7.1\end{array}$ \\
\hline Month & $\cdots$ & $\cdots$ & $\cdots$ & 10.7 & 5.8 & 8.4 & 8.0 & 5.6 & 6.6 \\
\hline
\end{tabular}


Table 38. Daily maximum, minimum, and mean dissolved oxygen concentrations at station 392125081193601, from the Willow Island Dam (downstream) continuousrecording water-quality monitor, June to october 1995 - - Continued.

$[\cdots$, value not determined]

Dissolved oxygen concentration, in milligrams per liter

\begin{tabular}{|c|c|c|c|c|c|c|c|c|c|}
\hline \multirow[t]{2}{*}{ Day } & \multicolumn{3}{|c|}{ August } & \multicolumn{3}{|c|}{ September } & \multicolumn{3}{|c|}{ October } \\
\hline & Maximum & Minimum & Mean & Maximum & Minimum & Mean & Maximum & Minimum & Mean \\
\hline $\begin{array}{l}1 \\
2 \\
3 \\
4 \\
5\end{array}$ & $\begin{array}{l}7.2 \\
7.3 \\
7.1 \\
7.0 \\
7.0\end{array}$ & $\begin{array}{l}6.9 \\
7.0 \\
6.8 \\
6.7 \\
6.8\end{array}$ & $\begin{array}{l}7.0 \\
7.2 \\
7.0 \\
6.9 \\
6.9\end{array}$ & $\begin{array}{l}6.5 \\
6.3 \\
6.7 \\
7.0\end{array}$ & $\begin{array}{l}\cdots .2 \\
6.0 \\
6.0 \\
6.3\end{array}$ & $\begin{array}{l}6.3 \\
6.2 \\
6.3 \\
6.6\end{array}$ & $\begin{array}{l}7.4 \\
7.4 \\
7.7 \\
7.2 \\
7.5\end{array}$ & $\begin{array}{l}7.1 \\
7.2 \\
7.2 \\
7.0 \\
7.1\end{array}$ & $\begin{array}{l}7.3 \\
7.2 \\
7.4 \\
7.1 \\
7.2\end{array}$ \\
\hline $\begin{array}{r}6 \\
7 \\
8 \\
9 \\
10\end{array}$ & $\begin{array}{l}7.0 \\
6.9 \\
7.1 \\
7.1 \\
6.6\end{array}$ & $\begin{array}{l}6.8 \\
6.2 \\
6.3 \\
6.6 \\
6.3\end{array}$ & $\begin{array}{l}6.9 \\
5.5 \\
6.7 \\
5.9 \\
6.5\end{array}$ & $\begin{array}{l}6.9 \\
6.6 \\
6.5 \\
6.6 \\
6.7\end{array}$ & $\begin{array}{l}6.5 \\
6.1 \\
6.2 \\
6.3 \\
6.3\end{array}$ & $\begin{array}{l}6.7 \\
6.4 \\
6.3 \\
6.5 \\
6.5\end{array}$ & $\begin{array}{l}7.6 \\
7.6 \\
7.5 \\
7.6 \\
7.6\end{array}$ & $\begin{array}{l}7.4 \\
7.4 \\
7.4 \\
7.3 \\
7.4\end{array}$ & $\begin{array}{l}7.5 \\
7.5 \\
7.4 \\
7.4 \\
7.5\end{array}$ \\
\hline $\begin{array}{l}11 \\
12 \\
13 \\
14 \\
15\end{array}$ & $\begin{array}{l}6.4 \\
6.4 \\
6.7 \\
7.1 \\
7.0\end{array}$ & $\begin{array}{l}6.1 \\
6.1 \\
6.2 \\
6.6 \\
6.6\end{array}$ & $\begin{array}{l}6.2 \\
6.3 \\
6.4 \\
6.9 \\
6.8\end{array}$ & $\begin{array}{l}6.7 \\
6.7 \\
6.7 \\
6.6 \\
6.8\end{array}$ & $\begin{array}{l}6.5 \\
6.5 \\
6.4 \\
6.5 \\
6.5\end{array}$ & $\begin{array}{l}6.6 \\
6.6 \\
6.5 \\
6.5 \\
6.7\end{array}$ & $\begin{array}{l}7.7 \\
7.7 \\
7.6 \\
--. \\
7.7\end{array}$ & $\begin{array}{l}7.5 \\
7.4 \\
7.3 \\
--. \\
7.3\end{array}$ & $\begin{array}{l}7.6 \\
7.5 \\
7.5 \\
-.- \\
7.5\end{array}$ \\
\hline $\begin{array}{l}16 \\
17 \\
18 \\
19 \\
20\end{array}$ & $\begin{array}{l}7.4 \\
7.5 \\
7.1 \\
7.3 \\
7.2\end{array}$ & $\begin{array}{l}6.9 \\
6.9 \\
6.7 \\
6.5 \\
6.7\end{array}$ & $\begin{array}{l}7.1 \\
7.1 \\
6.9 \\
6.9 \\
6.9\end{array}$ & $\begin{array}{l}6.8 \\
6.6 \\
6.6 \\
6.7 \\
6.7\end{array}$ & $\begin{array}{l}6.5 \\
6.5 \\
6.5 \\
6.5 \\
6.3\end{array}$ & $\begin{array}{l}6.6 \\
6.6 \\
6.6 \\
6.6 \\
6.5\end{array}$ & $\begin{array}{l}7.6 \\
7.7 \\
7.7 \\
7.5 \\
7.5\end{array}$ & $\begin{array}{l}7.3 \\
7.3 \\
7.3 \\
7.2 \\
7.3\end{array}$ & $\begin{array}{l}7.5 \\
7.5 \\
7.5 \\
7.4 \\
7.4\end{array}$ \\
\hline $\begin{array}{l}21 \\
22 \\
23 \\
24 \\
25\end{array}$ & $\begin{array}{l}6.9 \\
6.9 \\
6.7 \\
6.4 \\
\ldots .\end{array}$ & $\begin{array}{l}6.5 \\
6.4 \\
6.4 \\
6.1 \\
-. .\end{array}$ & $\begin{array}{l}6.7 \\
6.6 \\
6.5 \\
6.2 \\
\ldots .\end{array}$ & $\begin{array}{l}-.- \\
6.6 \\
6.9 \\
6.9 \\
7.2\end{array}$ & $\begin{array}{l}6.1 \\
6.6 \\
6.7 \\
6.9\end{array}$ & $\begin{array}{l}6.3 \\
6.7 \\
6.8 \\
7.0\end{array}$ & $\begin{array}{l}7.5 \\
7.4 \\
7.7 \\
7.7 \\
7.8\end{array}$ & $\begin{array}{l}7.3 \\
7.3 \\
7.4 \\
7.5 \\
7.6\end{array}$ & $\begin{array}{l}7.4 \\
7.4 \\
7.5 \\
7.6 \\
7.7\end{array}$ \\
\hline $\begin{array}{l}26 \\
27 \\
28 \\
29 \\
30 \\
31\end{array}$ & $\begin{array}{l}\cdots \\
\cdots \\
\cdots \\
\cdots \\
\cdots \\
\cdots\end{array}$ & $\begin{array}{l}\cdots \\
\cdots \\
\cdots \\
\cdots \\
\cdots \\
\cdots\end{array}$ & $\begin{array}{l}\cdots \\
\cdots \\
\cdots \\
- \\
\cdots \\
\cdots \\
-\end{array}$ & $\begin{array}{l}7.0 \\
6.8 \\
7.1 \\
7.1 \\
7.2 \\
\ldots .-\end{array}$ & $\begin{array}{l}6.5 \\
6.4 \\
6.8 \\
6.9 \\
6.9 \\
\ldots .\end{array}$ & $\begin{array}{l}6.8 \\
6.5 \\
6.9 \\
7.0 \\
7.0 \\
\ldots .\end{array}$ & $\begin{array}{l}7.8 \\
7.8 \\
-. . \\
8.1 \\
8.0 \\
8.1\end{array}$ & $\begin{array}{l}7.7 \\
7.6 \\
\cdots . \\
7.8 \\
7.9 \\
7.9\end{array}$ & $\begin{array}{l}7.8 \\
7.7 \\
-. . \\
8.0 \\
8.0 \\
8.0\end{array}$ \\
\hline
\end{tabular}

Month 
CONVERSION FACTORS AND ABBREVIATIONS

\begin{tabular}{rcl}
\hline \multicolumn{1}{c}{ Multiply } & By & To obtain \\
\hline inch (in.) & 25.4 & millimeter \\
foot $(\mathrm{f})$ & 0.3048 & meter \\
foot per mile $(\mathrm{ft} / \mathrm{mi})$ & 0.1894 & meter per kilometer \\
mile $(\mathrm{mi})$ & 1.609 & kilometer \\
square mile $\left(\mathrm{mi}^{2}\right)$ & 2.590 & square kilometer \\
\hline
\end{tabular}

Temperature is given in degrees Celsius $\left({ }^{\circ} \mathrm{C}\right)$, which can be converted to degrees Fahrenleit $\left({ }^{\circ} \mathrm{F}\right)$ by use of the following equation:

$$
\mathrm{F}=1.8\left({ }^{\circ} \mathrm{C}\right)+32
$$

River Mile: A unit of length applied to the main stem of a river to denote location. Typically, the mouth of a river is designated river mile zero and river mile length is measured upstream from this point. River mile zero on the Ohio River has been designated as the river's origin in Pittsburgh, Pa., however, and river mile length is measured downstream from this point.

Abbreviated water-quality units used in this report: Chemical concentrations and water temperature are given in metric units. Chemical concentration is given in grams per liter $(\mathrm{g} / \mathrm{L})$, milligrams per liter $(\mathrm{mg} / \mathrm{L})$, or micrograms per liter $(\mu \mathrm{g} / \mathrm{L})$. Milligrams per liter is a unit expressing the concentration of chemical constituents in solution as weight (milligrams) of solute per unit volume (liter) of water. One thousand milligrams per liter is equivalent to one gram per liter. One thousand micrograms per liter is equivalent to one milligram per liter. For concentrations less than $7,000 \mathrm{mg} / \mathrm{L}$, the numerical value is the same as for concentrations in parts per million.

Specific conductance of water is expressed in microsiemens per centimeter at 25 degrees Celsius $(\mu \mathrm{S} / \mathrm{cm})$. This unit is equivalent to micromhos per centimeter at 25 degrees Celsius $(\mu \mathrm{mho} / \mathrm{cm})$, formerly used by the U.S. Geological Survey. 